\title{
Räumliche Entscheidungsfindung mit Hilfe raumbezogener Informationssysteme
}

\author{
Konzepte und Anwendungsmöglichkeiten \\ für geographische Informationen zur Lösung \\ von räumlichen Entscheidungsproblemen \\ am Beispiel der Forstwirtschaft
}

\section{Dissertation}

zur Erlangung des Doktorgrades

der Fakultät für Forstwissenschaften und Waldökologie

der Georg August Universität Göttingen

vorgelegt von JAROSLAV MYŠIAK

geboren in Žilina/Slowakei

Göttingen 2000 


\section{D7}

1. Berichterstatter: Prof. Dr. Dr. h.c. Branislav Sloboda

2. Berichterstatter: Prof. Dr. Bernhard Möring

3. Berichterstatter: $\quad$ PD Dr. Winfried Kurth

Tag der mündlichen Prüfung: 20. 04. 2001

Erschienen im Internet unter: http://webdoc.sub.gwdg.de/diss/2001/mysiak/ 


\section{Danksagung}

Die vorliegende Arbeit entstand während meines dreijährigen Forschungsaufenthalts im Institut für Forstliche Biometrie und Informatik der Georg - August Universität Göttingen unter Beaufsichtigung von Herrn Prof. Dr. Dr.h.c. Branislav Sloboda. Ihm als Promotor und Betreuer der Arbeit gilt mein herzlicher Dank.

Bei den Herren Prof. Dr. S. Šmelko, Doz. Dr. L'. Scheer und Dr. J. Ďurský aus dem Institut für Forsteinrichtung und Geodäsie der Technischen Universität Zvolen/Slowakei, die am Anfang der Arbeit standen und das Promotionsvorhaben unterstützt haben, möchte ich mich ganz herzlich bedanken.

Für breite Unterstützung, informativ-kritische Gespräche sowie ermutigende Worte bin ich Herrn Prof. Dr. J. Saborowski und Herrn Dr. M. Jansen zum Dank verpflichtet. Meinen Freunden und Kollegen vorwiegend aus dem Institut für Forstliche Biometrie und Informatik, vor allem V. Mues, R. Schulz, S. Hentschel, A. Wameling und J. Šálka, die durch konstruktiven Rat, anregende Diskussionen sowie freundliche Arbeitsatmosphäre wesentlich zu dieser Arbeit beigetragen haben, gilt ebenfalls mein Dank.

Die Arbeit war durch eine finanzielle Unterstützung des Deutschen Akademischen Austauschdienstes DAAD ermöglicht, wofür ich mich herzlich bedanken möchte. 


\section{Inhaltverzeichnis}

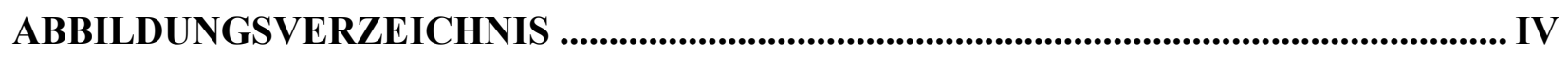

TABELLENVERZEICHNIS ........................................................................................................ VI

1 EINLEITUNG, ZIELE UND STRUKTUR DER ARBEIT .............................................2

2 FORSTLICHES PLANUNGS- UND ENTSCHEIDUNGSMODELL ...............................7

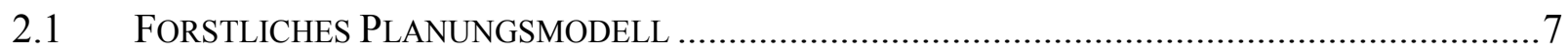

2.2 ENTSCHEIDUNGSFINDUNG IN DER FORSTWIRTSCHAFT …….........................................

2.3 RÄUMLICHE ENTSCHEIDUNGEN IN DER FORSTWIRTSCHAFT ……..................................... 11

2.4 ABLEITUNG EINER RÄUMLICHEN ENTSCHEIDUNGSSITUATION.......................................15

2.5 ZuSAMMENFASSUNG DER MOtIVATION UND DES VORHABENS DER ARBEIT ......................17

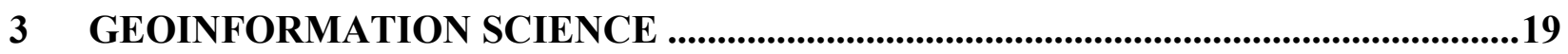

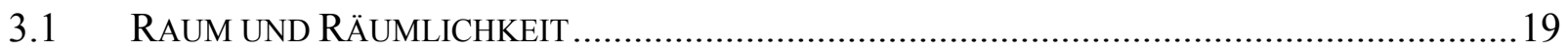

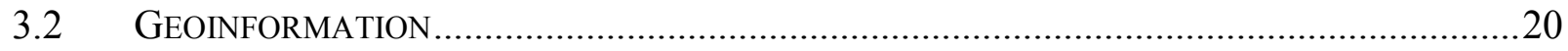

3.3 POLITISCHE, WIRTSCHAFTLICHE UND WISSENSCHAFTLICHE DIMENSIONEN DER

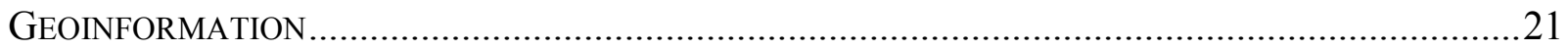

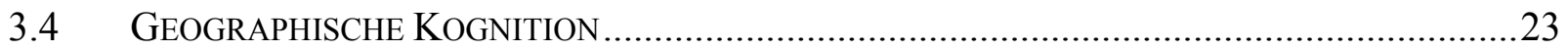

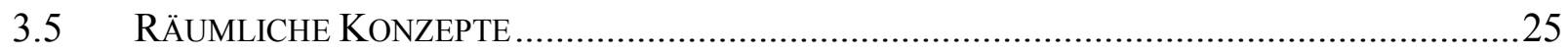

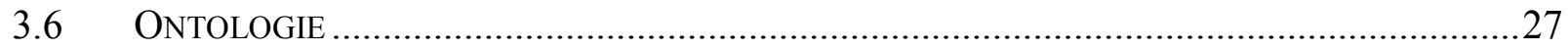

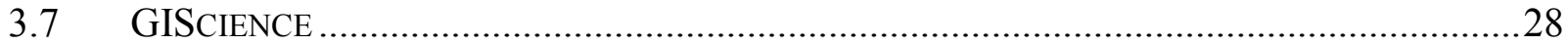

4 GEOGRAPHISCHE INFORMATIONSTECHNOLOGIE UND -VERARBEITUNG 30

4.1 GEOGRAPHISCHES INFORMATIONSSYSTEM (GISYSTEM) .............................................30

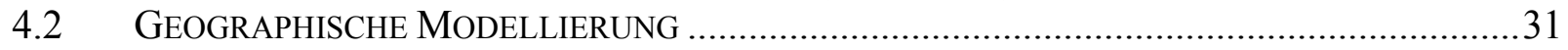

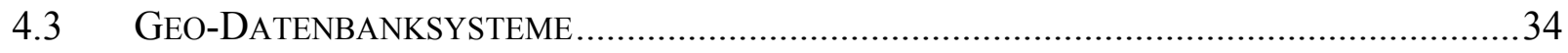

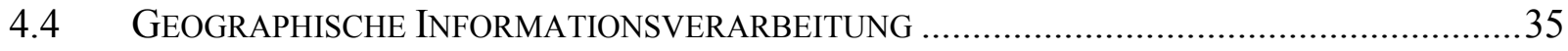

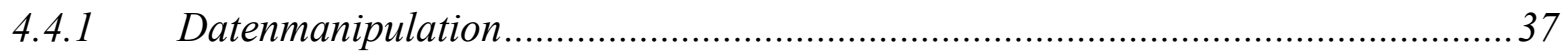

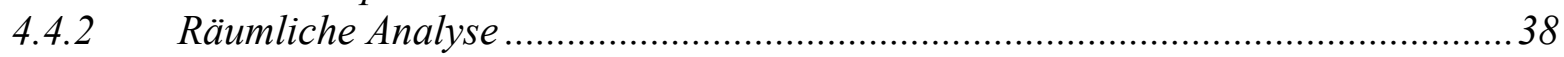

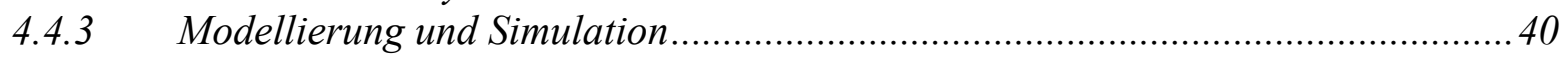

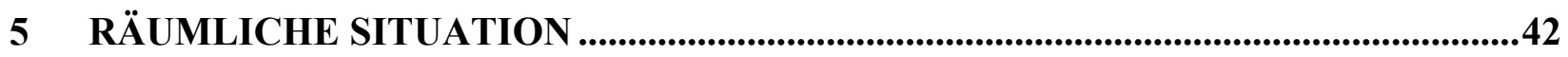

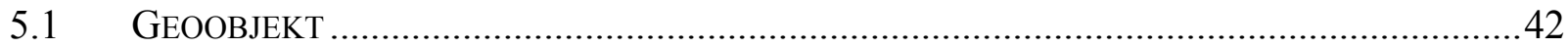

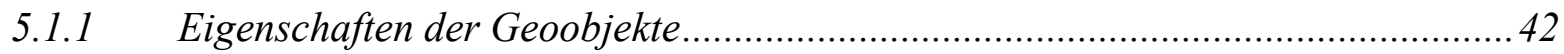

5.1.2 Geometrische Eigenschaften eines Geoobjektes ................................................4 44

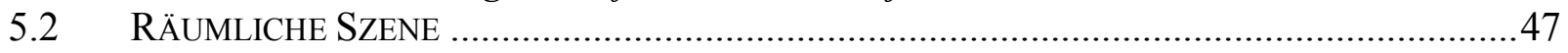

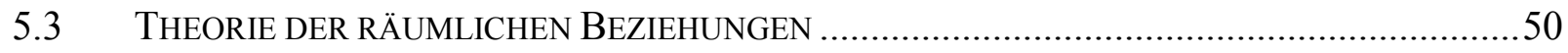

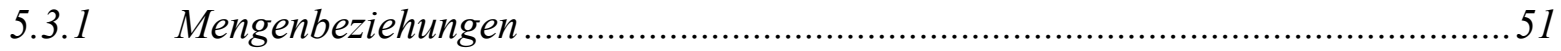

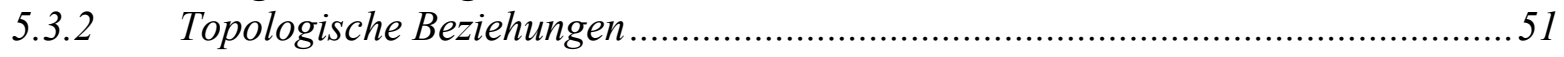

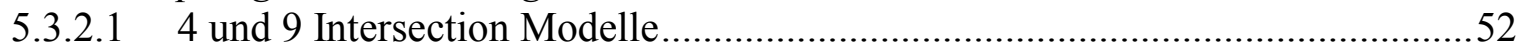

5.3.2.2 Calculus based Modell ..............................................................................54

5.3.2.3 RCC topologische Relationen .....................................................................5

5.3.2.4 Modell der Grundfläche und der generischen Fläche ........................................55

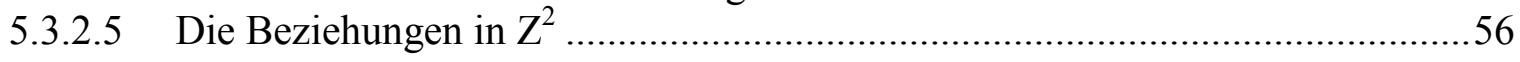




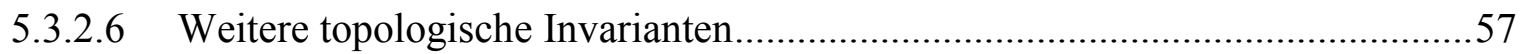

5.3.2.7 Beziehungen für Polygone mit Löchern...........................................................6

5.3.2.8 Quantifikation der topologischen Beziehungen ................................................61

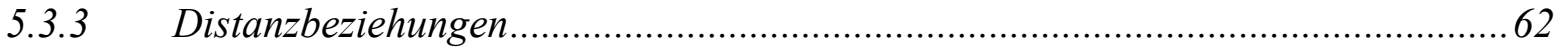

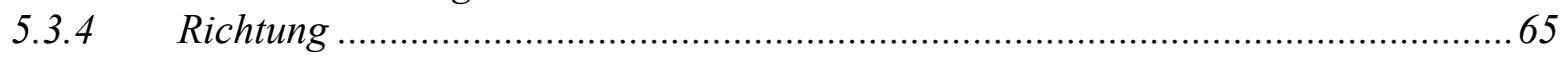

5.3.4.1 Relationen der konischen Bereiche (Kegelform-Ansatz)...................................67

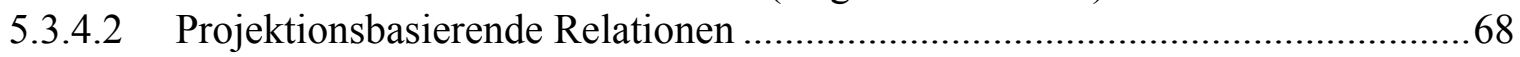

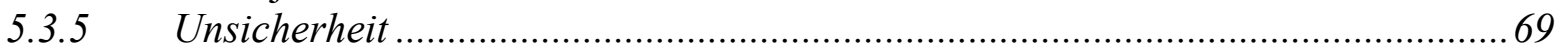

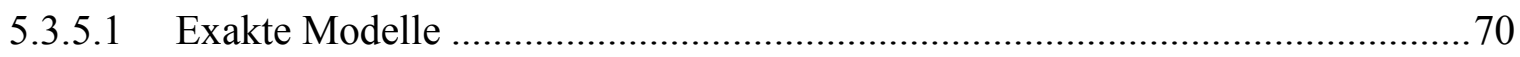

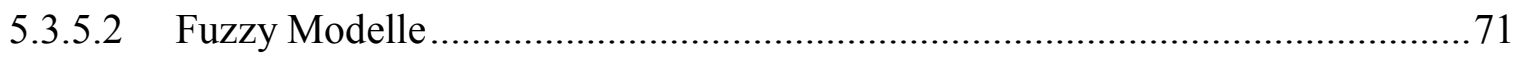

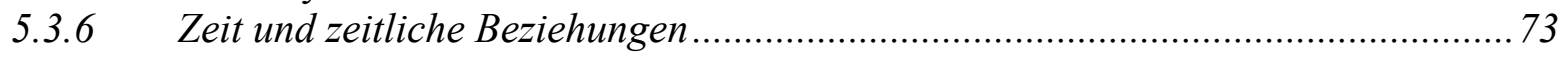

5.3.6.1 Zeitmodelle............................................................................................74

5.3.6.2 Taxonomie der spatial-temporal Prozesse..........................................................75

5.3.6.3 Zeitliche Beziehungen...................................................................................76

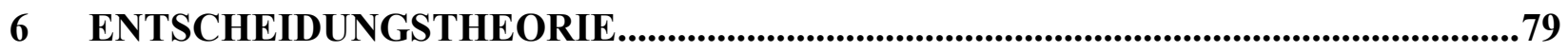

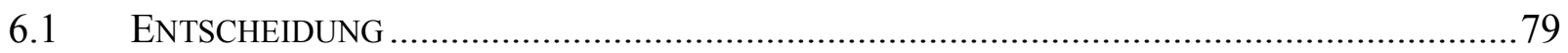

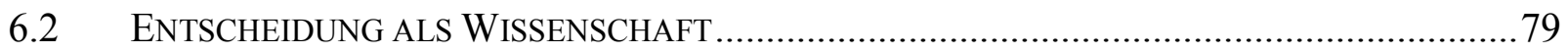

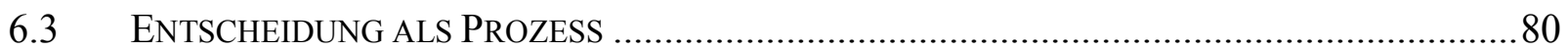

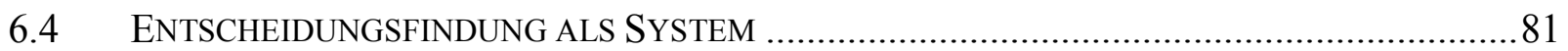

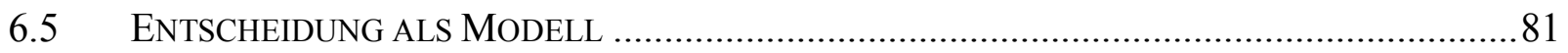

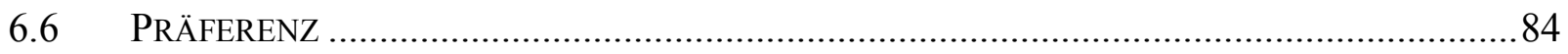

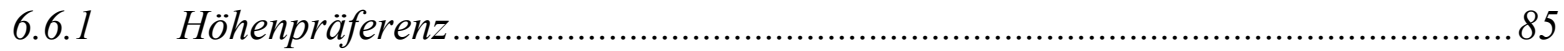

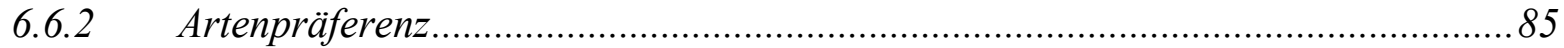

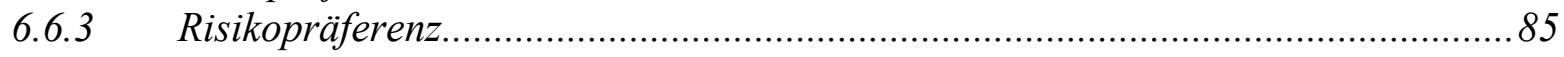

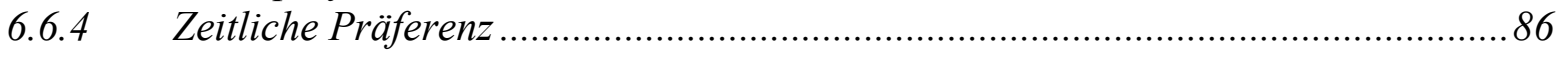

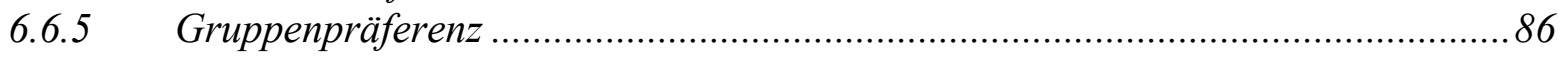

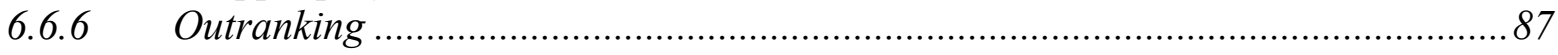

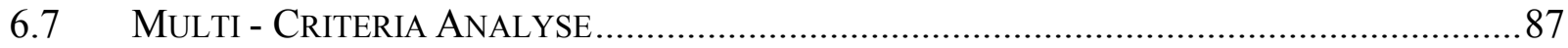

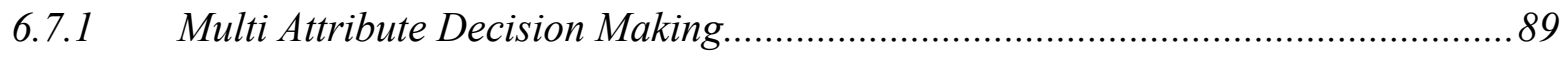

6.7.2 Multi-objective decision making MODM .......................................................90

6.8 ENTSCHEIDUNGSUNTERSTÜTZUNGSSYSTEME........................................................92

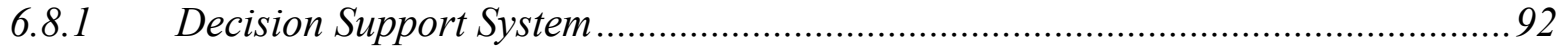

6.8.2 GDSS (Group decision support systems) ……..............................................94

7 RÄUMLICHE ENTSCHEIDUNGEN.................................................................................97

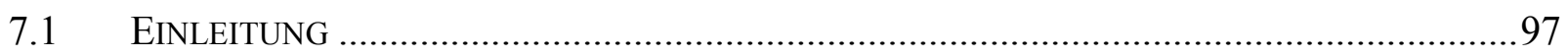

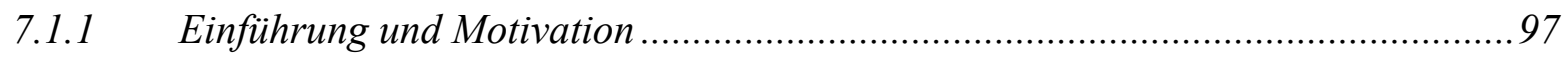

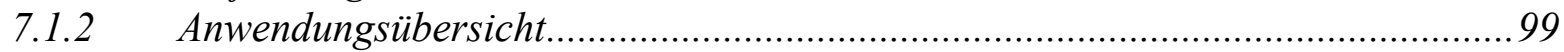

7.1.3 Stand der Forschung und Literaturübersicht .................................................100

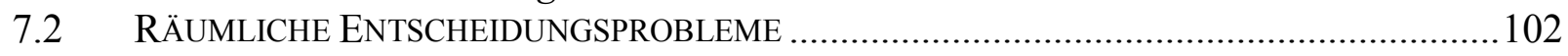

7.3 MODELLIERUNG RÄUMLICHER ENTSCHEIDUNGSSITUATION …………..........................105

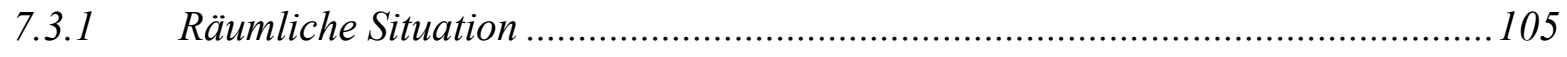

7.3.2 Räumliche Entscheidungssituation ..............................................................107

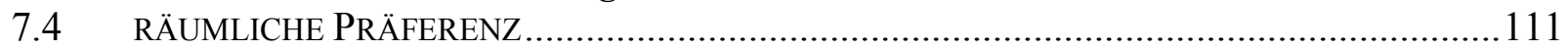

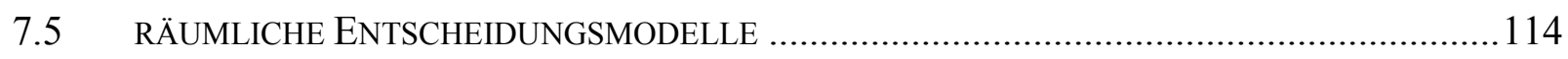

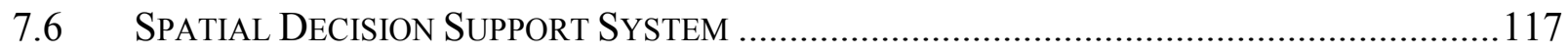




\section{ANWENDUNG DER GEOINFORMATIONEN ZUR ENTSCHEIDUNGSFINDUNG}

IN DER FORSTWIRTSCHAFT ..........................................................................119

8.1 BEITRAG DER GISCIENCE ZUR FORSTWIRTSCHAFT ....................................................119

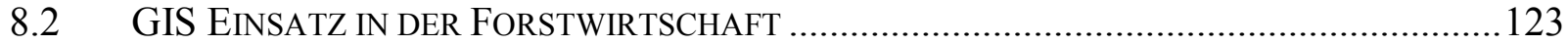

8.3 RÄUMLICHE HANDLUNGSSITUATION IN DER FORSTWIRTSCHAFT ..................................126

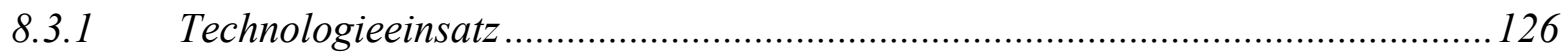

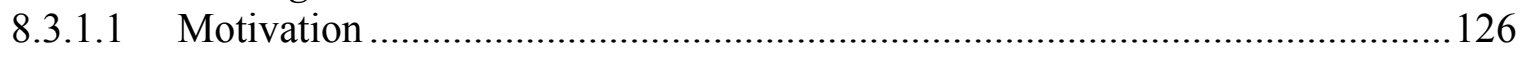

8.3.1.2 Modellierung der räumlichen Situation.........................................................127

8.3.1.3 Entscheidungsfindung ............................................................................... 130

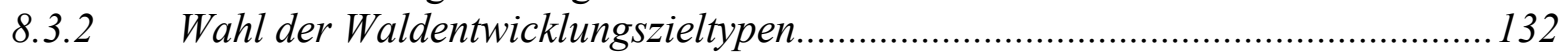

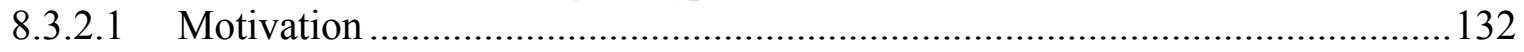

8.3.2.2 Modellierung der räumlichen Situation........................................................132

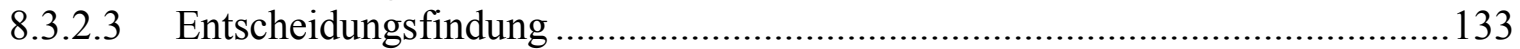

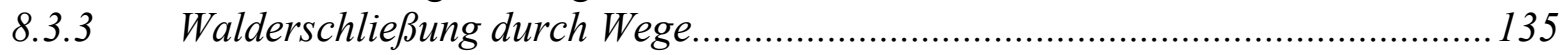

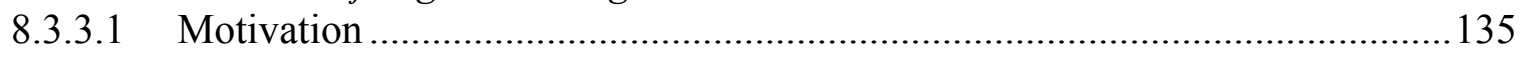

8.3.3.2 Modellierung der räumlichen Situation..........................................................136

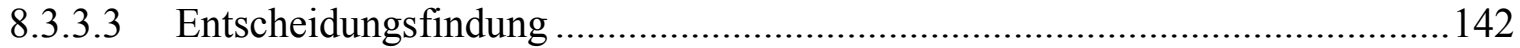

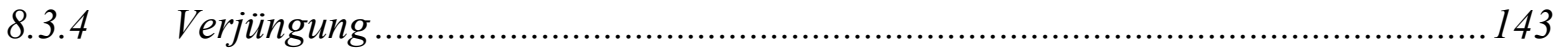

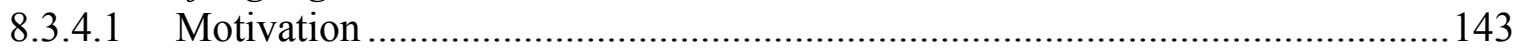

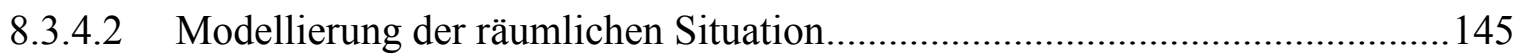

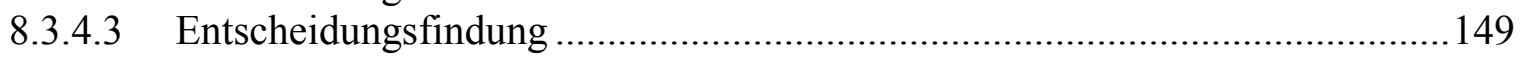

8.4 SCHLUSSFOlgERUNG ZUR ANWENDUNG DER GEOINFORMATIONEN IN DER

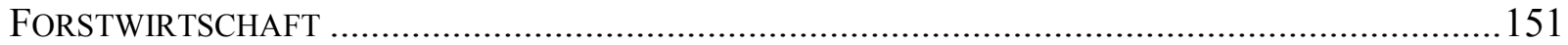

\section{ANWENDUNG RÄUMLICHER ENTSCHEIDUNGSFINDUNG AM BEISPIEL DES}

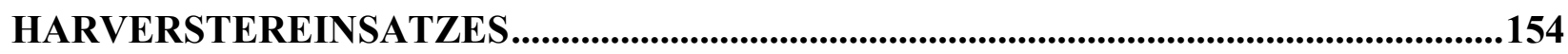

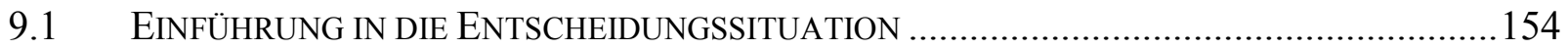

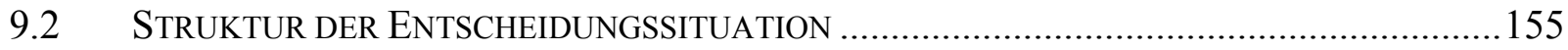

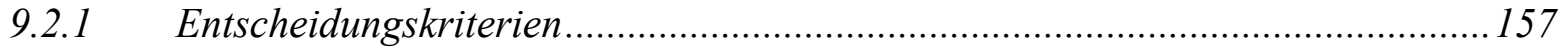

9.2.1.1 Nicht kompensierbare Bedingungen ...............................................................157

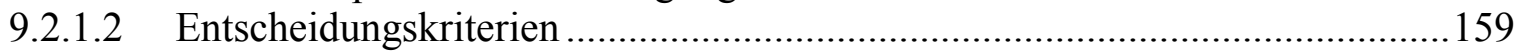

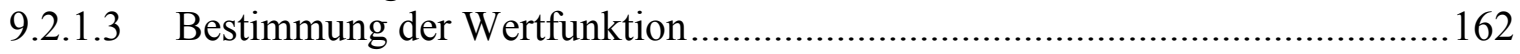

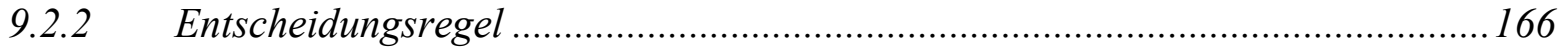

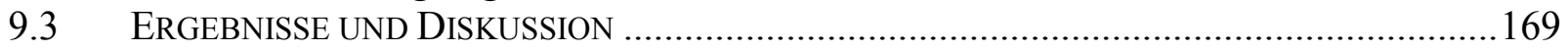

10 DISKUSSION UND ZUSAMMENFASSUNG ...............................................................174

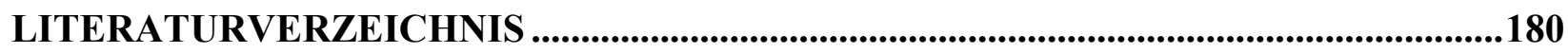




\section{Abbildungsverzeichnis}

Abb. 1: Grundlegende Themenbereiche der Arbeit. $\quad 2$

Abb. 2: Semantischer Aufbau der Arbeit. $\quad 4$

Abb. 3: Aufbau der Arbeit nach Kapiteln geordnet.

Abb. 4: Formale Gliederung der Arbeit. $\quad 6$

Abb. 5: Schematische Darstellung forstlicher Entscheidungen. $\quad 10$

Abb. 6: Interdependenzen der räumlichen Ordnung. 12

Abb. 7: Beispiele räumlicher Konzepte.

Abb. 8: Konzepte der Field-Repräsentation.

Abb. 9: Entwicklung Geographischer Informationssysteme. 30

Abb. 10: Komponenten eines Geoobjektes. $\quad 42$

Abb. 11: Beispiele komplexer Geometrie eines Geoobjektes. 43

Abb. 12: Progressive, konservative und generalisierende Approximation eines Geoobjektes. 44

Abb. 13: Darstellung einer räumlichen Szene. $\quad 48$

Abb. 14: Ähnlichkeit der räumlichen Beziehungen. $\quad 48$

Abb. 15: Beispiele der Szenebeschreibung. $\quad 49$

Abb. 16: Topologische Beziehungen zwischen Polygonen nach 4-Intersections Modell. 52

Abb. 17: Konzeptioneller Nachbarschaftsgraph der topologischen Beziehungen zwischen einfachen Polygonen. 53

Abb. 18: Topologische Beziehungen zwischen Linien nach der Inhaltsinvariante. 53

Abb. 19: Topologische Beziehungen zwischen einer Linie und einem Polygon. 54

Abb. 20: Topologische Beziehungen nach dem RCC Ansatz.

Abb. 21: Modell der topologischen Beziehungen nach WORBOYS \& BOFAKOS (1993). 56

Abb. 22: Topologische Beziehungen zwischen den Objekten in Raster Tesselation. 57

Abb. 23: Komponenteinvarianten als detailliertere topologische Modelle. 59

Abb. 24: Ableitung topologischer Beziehungen zwischen Geoobjekten mit Löchern. 60

Abb. 25: Metrische Konzepte zur Beschreibung von topologischen Beziehungen. 62

Abb. 26: Unterschiedliche Typen der Distanzbeziehungen.

Abb. 27: Qualitative Entfernungsbeziehungen.

Abb. 28: Ansätze der Richtungsbeziehungen. 66

Abb. 29: Schwierigkeiten der Ableitung der Richtungsbeziehungen für räumlich ausgedehnte Objekte. $\quad 67$

Abb. 30: DRM Beziehungen eines Geoobjektes. 69

Abb. 31: Unsicherheitsmodelle der räumlich ausgedehnten Geoobjekte. $\quad 70$

Abb. 32: Konzept der fuzzy-unscharfen Grenze eines räumlichen Objektes.

Abb. 33: Prozesse der Änderung von räumlichen Objekten.

Abb. 34: Zeitbeziehungen des linearen Zeittyps.

Abb. 35: Zeitbeziehungen in dem zyklischen Zeittyp.

Abb. 36: Zusätzliche Zeitbeziehungen im verzweigten Zeittyp. $\quad 78$

Abb. 37: Struktur eines Entscheidungsproblem nach dem 5 Komponenten Modell. 79

Abb. 38: Struktur einer Entscheidungssituation. $\quad 82$

Abb. 39: Grundmodell der Entscheidungstheorie.

Abb. 40: Beziehungen der räumlichen Entscheidungsfindung. 98

Abb. 41: Schematische Erweiterung eines Entscheidungsproblems um die Räumlichkeit. 102

Abb. 42: Prozess der räumlichen Entscheidungsfindung. 103

Abb. 43: Räumliche Entscheidungsprobleme und zugrundeliegende Konzepte. 103

Abb. 44: Dimension der Entscheidungskriterien. $\quad 104$

Abb. 45: Bestandteile einer räumlichen Situation. 105

Abb. 46: Eigenschaften eines räumlichen Elementes und einer räumlichen Szene. 106

Abb. 47: Beziehungsarten einer räumlichen Situation. 106

Abb. 48: Integration von unterschiedlichen Konzepten einer räumlichen Situation. 107

Abb. 49: Bestandteile räumlicher Entscheidungssituationen. 107

Abb. 50: Räumliche Beziehungen als Ausprägungen räumlicher Kriterien. 108

Abb. 51: Zusammensetzung der Entscheidungsprobleme. 109

Abb. 52: Entscheidungsraum (links) und Ergebnisraum (rechts) einer räumlichen Entscheidungssituation. 110

Abb. 53: Graphische Hilfsmittel zur Erforschung von Zusammenhängen in der Entscheidungssituation. $\quad 110$

Abb. 54: Modellierung räumlicher Präferenz. 112 
Abb. 55: Schematische Darstellung der positionalen Entscheidungsprobleme.

Abb. 57: Mögliche topologische Beziehungen zwischen den geeigneten Einsatzorten und dem Bestand/Standort. 128

Abb. 58: Mögliche topologische Beziehungen zwischen den Wegen und polygonhaften räumlichen Objekten. 128

Abb. 59: Komposition von Zellen der Geländerepräsentation.

Abb. 60: Konzept des räumlichen Musters.

Abb. 61: Dimensionen der Musterbeschreibung.

Abb. 62: Beziehungstabellen einzelner ,Dimensionen' der Musterbeschreibung.

Abb. 63: Räumliche Konzepte der Handlungssituation für den Technologieeinsatz.

Abb. 64: Mögliche Designs der Entscheidungssituation.

Abb. 65: Räumliche Konzepte und lokale Umgebung.

Abb. 66: Mögliche Interdependenzen in der Entscheidungssituation.

134

Abb. 67: Design 1 der Entscheidungssituation und seine möglichen Modifikationen. 134

Abb. 68: Design 2 und 3 der Entscheidungssituation. 135

Abb. 69: Wegenetz als Graph.

136

Abb. 70: Einschränkung der topologischen Beziehungen eines Graphen.

137

Abb. 71: Beziehungen eines Pfades mit linienartigen räumlichen Objekten.

Abb. 72: Beziehungen eines Pfades zu polygonhaften Objekten.

Abb. 73: Weitere topologische Beziehungen des Graphen.

139

Abb. 74: Metrische Verfeinerung der räumlichen Konzepte.

Abb. 75: Unterschiedliche Beziehungen eines Graphen und einer Tesselation.

Abb. 76: Richtungsmodelle des Graphen.

Abb. 77: Richtungsbeziehungen einer Kante bezogen auf eine vorgegebene Richtung. 140

Abb. 78: Distanzbeziehungen eines Graphen.

141

Abb. 79: Konzepte der Formbeschreibungen eines Pfades.

141

Abb. 80: Schritte der Alternativengenerierung.

142

Abb. 81: Entscheidungssituation über Walderschließung. 143

Abb. 82: Konzepte der Walderneuerung.

Abb. 83: Komplexität der Walderneuerung am Beispiel des Saumfemelschlags.

Abb. 84: Relevante Beziehungen der räumlichen Situation bei Walderneuerung.

Abb. 85: Topologische Beziehungen der Inhaltsinvariante zwischen Verjüngungsfläche und der Teilfläche. 146

Abb. 86: Topologische Beziehungen der Teilflächen zueinander nach der Inhaltsinvariante. 147

Abb. 87: Darstellung der räumlichen Beziehungen zwischen den Teilflächen und der Verjüngungseinheit. 147

Abb. 88: Beziehungen der Teilflächen zueinander.

Abb. 89: Räumliche Beziehungen bezüglich der Höhe (z Koordinate).

Abb. 90: Deckungsschutzsysteme für das Fachwerk.

Abb. 91: Entscheidungssituation der Verjüngungsplanung.

Abb. 92: Schematische Darstellung der räumlichen Entscheidungsunterstützung bei Verjüngungsplanung. $\quad 150$

Abb. 93: Objekt- und Anordnung - Suche.

Abb. 94: Die für die Fragestellung entscheidungsrelevanten Geoinformationen des Betriebsbezirks Lichtenhagen.154

Abb. 95: Die bei der Suche nach Einsatzorten für den Harvester zu berücksichtigenden Entscheidungskriterien. 155

Abb. 96: Semantischer Aufbau der Entscheidungssituation und Ableitung der Entscheidungskriterien. $\quad 156$

Abb. 97: Ableitung potentieller Einsatzorte.

158

Abb. 98: Konzepte der Konvexität räumlicher Objekte.

160

Abb. 99: Konzeption der Erschließung von Einsatzorten.

161

Abb. 100: Konzept der Konzentration als Dispersion ihrer Präferenzen für den Einsatz eines Harvesters. 161

Abb. 101: Unterschiedliche Typen der Wertfunktion zur Abbildung der Präferenzen.

162

Abb. 102: Kompensierbare ,weiche' Entscheidungskriterien (factors) und ihre räumliche Umsetzung.

163

Abb. 103: Konzepte der Gewichtszuordnung bei der Nutzwertanalyse und der Ordered Weighted Averaging.

167

Abb. 104: Entscheidungsstrategie beim Ordered Weighted Averaging.

Abb.105: Ergebnisse der Nutzwertanalyse.

170

Abb.106: Die Ergebnisse des Ordered Weighted Averaging.

171

Abb. 107 : Konzepte der räumlichen Konzentration als distanzbasierte Dispersion der Präferenzwerte. 172

Abb. 108: Der Einfluß der Konzentration von Einsatzorten auf die Entscheidung. 


\section{Tabellenverzeichnis}

Tab. 1: Beispiele der Bereiche, in welchen räumliche Entscheidungsfindung in der Forstwirtschaft traditionell Bedeutung hat.

Tab. 2: Universelle GIS Operationen.

Tab. 3: Topologische Beziehungen des dimension extended Modells.

Tab. 4: Die Beziehungen der Inhaltsinvariante, welche mit der Komponenteninvariante erweitert werden können. 60

Tab. 5: Topologische Entfernungen der 8 Polygon-Polygon Relationen..

Tab. 6: Konzeptionelle Zeitmodelle.

Tab. 7: Identitätsbezogene Objektänderung.

Tab. 8: Zyklische Zeitbeziehungen.

Tab. 9: Vergleich der MADM und MODM Verfahren. $\quad 88$

Tab. 10: Klassifikation von MADM - Methoden. $\quad 89$

Tab. 11: Klassifikation von MODM - Methoden. 91

Tab. 12: Abgrenzung der Entscheidungsunterstützungssysteme. 93

Tab. 13: Vergleich von mentalen und formalen Modellen. 97

Tab. 14: Aufgaben der räumlichen Entscheidungsfindung. 99

Tab. 15: Beispiele der Entscheidungssituationen, bei welchen die Räumlichkeit der Alternativen von besonderer $\begin{array}{ll}\text { Bedeutung ist. } & 102\end{array}$

Tab. 16: Individuelle Ereignisse der Entscheidungsprobleme. 115

Tab. 17: Ereignisse, welche die lokale Konfiguration der Alternativen ändern. 115

Tab. 18: Gegenüberstellung der GIScience und der Forstwissenschaft. $\quad 119$

Tab. 19: Anwendungsbeispiele von GIS in der Forstwirtschaft. 124

Tab. 20: Hervorgehobene Merkmale der räumlichen Entscheidungsprobleme. 126

Tab. 21: Entscheidungsrelevante räumliche Beziehungen bei Technologieeinsatz. 127

Tab. 22: Konzepte der Walderschließung. 136

Tab. 23: Anzahl der möglichen topologischen Beziehungen eines Graphen nach der leer-nichtleeren Beschreibung der Schnittmengen. 138

Tab. 24: Entscheidungsrelevante Beziehungsarten der Entscheidungssituation $\quad 142$

Tab. 25: Konzepte des Waldaufbaus. $\quad 144$

Tab. 26: Konzepte der räumlichen Ordnung des Verjüngungsablaufes. $\quad 144$

Tab. 27: Zusammenfassung entscheidungsrelevanter Beziehungen von Verjüngungsfläche. 146

Tab. 28: Zusammenfassung der entscheidungsrelevanten Beziehungen von Teilflächen. 147

Tab. 29: Klassifizierungsregel zur Ausscheidung von Gefährdungsstufen von Waldstandorten. 157

Tab. 30: Die entscheidungsrelevanten topologischen Beziehungen der Einsatzorte und Bestände und ihre mögliche Quantifikation.

Tab. 31: Gewichte (Artenpräferenz) die zur Aggregation der Entscheidungskriterien in den Entscheidungsregeln verwendet wurde. 


\section{Einleitung, Ziele und Struktur der Arbeit}

Die vorliegende Arbeit befasst sich mit dem Thema der räumlichen Entscheidungsfindung an Beispielen aus der Forstwirtschaft: Sie untersucht die räumliche Entscheidungssituation bezüglich räumlicher (geographischer) Konzepte, Strukturierung und Kategorisierung räumlicher Entscheidungsprobleme und räumlicher Entscheidungsfindung sowie -unterstützung.

Räumliche Entscheidungsfindung ist ein aktuelles Thema von Praxis und Forschung in verschiedenen Wissenschaftsdisziplinen. Ihre Bedeutung ist vor allem auf die wachsende Komplexität der traditionellen Entscheidungssituation, welche durch die steigenden neuartigen Ansprüche der Gesellschaft verursacht wird, sowie auf den Fortschritt der Verarbeitung von räumlichen Informationen zurückzuführen. Die Notwendigkeit, räumliche Entscheidungen treffen $\mathrm{zu}$ müssen, ist nicht neu. Das Bedürfnis, sich im Raum zurechtzufinden und zielbezogen zu handeln, liegt jeder Handlung zugrunde. Das Streben nach bewusstem Umgang mit räumlichen Konzepten und Transparenz der Entscheidungsfindung in der räumlichen Domäne ${ }^{1}$ ist die Triebkraft des wachsenden Interesses an räumlicher Entscheidungsfindung.

Die Forstwirtschaft ist ein traditioneller Anwendungsbereich räumlicher Entscheidungsfindung. Sie strebt einen größtmöglichen, an der Zielsetzung gemessenen Nutzen in einem raum-zeitlichen Gefüge des Waldes an. Sie operiert im Raum, da Wald einen Raum darstellt, einen Raum von hoher Komplexität in Struktur und Aufbau. Die meisten forstlichen Entscheidungen betreffen den Raum, haben einen räumlichen Bezug oder räumliche Konsequenzen. Positionale Aspekte sind in der räumlichen Ordnung des Waldes und der Nutzungsplanung enthalten, haben Einfluss auf die Stabilität des Waldes und die Produktionssicherheit und spiegeln sich in den Kosten und Einnahmen der Bewirtschaftung wieder.

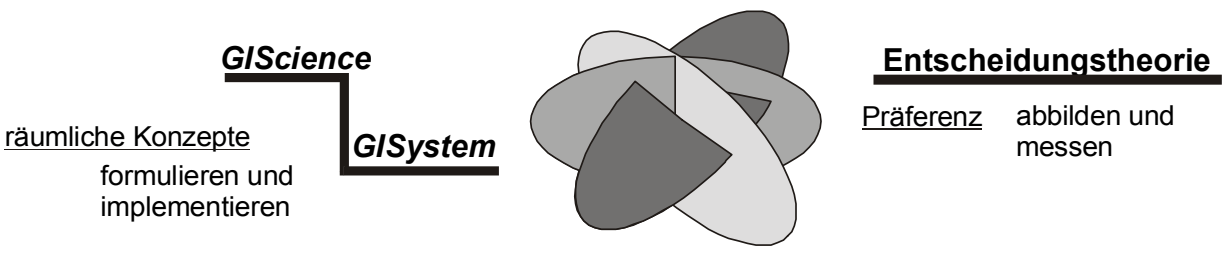

Forstwirtschaft

räumliche Konzepte und Präferenz anwenden

Abb. 1: Grundlegende Themenbereiche der Arbeit.

Darüber hinaus werden an den Wald neuartige, mit dem gesellschaftlichen Wandel verbundene Nutzungsansprüche erhoben, die ebenfalls einen ausgeprägten räumlichen Charakter besitzen (z.B. Erholungsfunktion des Waldes, Naturschutz und Landschaftspflege etc.). Räumlicher Entscheidungsfindung, d.h. dem Streben nach einer zielgerichteten Lösung der Entscheidungsprobleme in räumlichem Kontext, wird eine wachsende Bedeutung unter den Methoden der Fortseinrichtung eingeräumt.

1 Als Domäne wird in dieser Arbeit allgemein ein Spezialgebiet (Anwendungs- oder Grundbereich) verstanden (vgl. DUDEN 1989). 
Die Arbeit umfasst drei Felder, deren Überlappung, Interaktion und Anwendungspotential hierbei von Bedeutung sind. In Abbildung 1 sind die von der Arbeit betroffenen Bereiche schematisch dargestellt: Die neu konzipierte Wissenschaft der Geoinformation (GIScience), die Entscheidungstheorie und die Forstwirtschaft. Die GIScience untersucht räumliche Konzepte, welche in dem Entscheidungsprozeß zur Abbildung räumlicher Präferenz eines Entscheidungsträgers angewendet werden und die Entscheidungsfindung in einem räumlichen Anwendungsbereich ermöglichen.

GIScience (Geoinformationswesen) wurde als Wissenschaft über räumliche Konzepte formuliert (GoODCHILD et al. 1999). Ihre Aufgabe ist es, die Formulierung und Anwendung von räumlichen Konzepten zu unterstützen. Gegenstand ihrer Forschung ist die räumliche Information (Geoinformation). Das Verstehen und Verarbeiten von Geoinformationen sind mit den zugrundeliegenden räumlichen Konzepten untrennbar verbunden. Erhebung und Verarbeitung räumlicher Informationen verursachen einen erheblichen Aufwand, der durch ihre Nutzung wieder eingespielt werden soll. Ihr Nutzungswert wird von der Fähigkeit abgeleitet, zur Verbesserung einer Entscheidung beizutragen. Die in letzten Jahren zunehmende Aufmerksamkeit für die Geoinformationen ist auf das wachsende Bedürfnis zurückzuführen, räumliche Entscheidungen effektiv und transparent zu treffen. Aus diesem Grund wird der räumlichen Entscheidungsfindung auch innerhalb der GIScience große Bedeutung eingeräumt. Die räumliche Entscheidungsfindung wird auch als höhere Ebene der Geographischen Informationsverarbeitung (spatial data handling) verstanden.

Die räumliche Entscheidungsfindung ist ein Bestandteil der Entscheidungstheorie, ihr spezialisierter Zweig zur Lösung räumlicher Entscheidungsprobleme. Dabei werden räumliche Konzepte zur Abbildung der Präferenz eines Entscheidungsträgers angewendet. Eine Präferenz, welche die relative Lage und die räumlichen Konsequenzen der Alternativen zielbezogen anordnen kann, wird als räumliche Präferenz bezeichnet. Vor allem die Theorie räumlicher Beziehungen, ein zentraler Forschungsbereich der GIScience, ist für räumliche Entscheidungsfindung von großer Bedeutung. Anhand der positionsbezogenen Aspekte räumlicher Objekte können Unterschiede der Alternativen erfasst und bewertet werden.

Abbildung 2 beschreibt schematisch die von der Arbeit behandelten Themen aus ihrer inhaltlichen Sicht. Entscheiden heißt die Entscheidungssituation mit ihren Elementen und strukturellen Beziehungen zu modellieren, die zielbezogenen Konsequenzen der Alternativen zu bewerten und schließlich ihre unterschiedlichen Aspekte zu verknüpfen, um die Alternativen anordnen zu können. Haben die Elemente der Entscheidungssituation räumlichen Bezug und Eigenschaften, so muss der Räumlichkeit in allen diesen Schritten Rechnung getragen werden: Entscheidungsrelevante räumliche Konzepte müssen formuliert, bewertet und angeordnet werden.

Die geographische Kognition befasst sich mit Wahrnehmung, Gedächtnis, Handlung und Kommunikation raum-zeitlicher Eigenschaften der Objekte und Ereignisse in realer oder digitaler Repräsentation. Sie behandelt Themen, die für die räumliche Entscheidungsfindung von großer Bedeutung sind: Wie Menschen geographische Information aufnehmen und wie sie zur Entscheidungsfindung herangezogen wird, wie die Menschen räumliche Konzepte entwickeln und anhand dieser handeln und wie komplexe Geoinformation dargestellt werden kann, um den Verstand und die Entscheidungsfindung zu unterstützen. 
Digitale Geoinformationen entstehen während einer Abbildung der mentalen auf die formalen Modelle im Prozess der Datenmodellierung. Ihre rechengestützte Verarbeitung umfasst Analyse, Modellierung und Simulation, deren Ergebnisse in die Entscheidungsfindung einfließen.

Die räumlichen Eigenschaften entscheidungsrelevanter Objekte müssen abgewogen werden. Dabei werden vor allem die positionsbezogenen Aspekte (relative Position) wichtig, die anhand räumlicher Beziehungen wie Topologie, Distanz, Richtung und Hierarchie beschrieben werden können. Darüber hinaus können auch individuelle Eigenschaften wie Form, Größe, Ausdehnung und Ausrichtung zur Entscheidungsfindung beitragen.

Die anhand der räumlichen Konzepte abgebildeten Präferenzen werden mit Hilfe der Entscheidungsmodelle verknüpft. Somit wird eine Anordnung der Alternativen sowie eine transparente Darstellungsweise des Entscheidungsprozesses möglich.

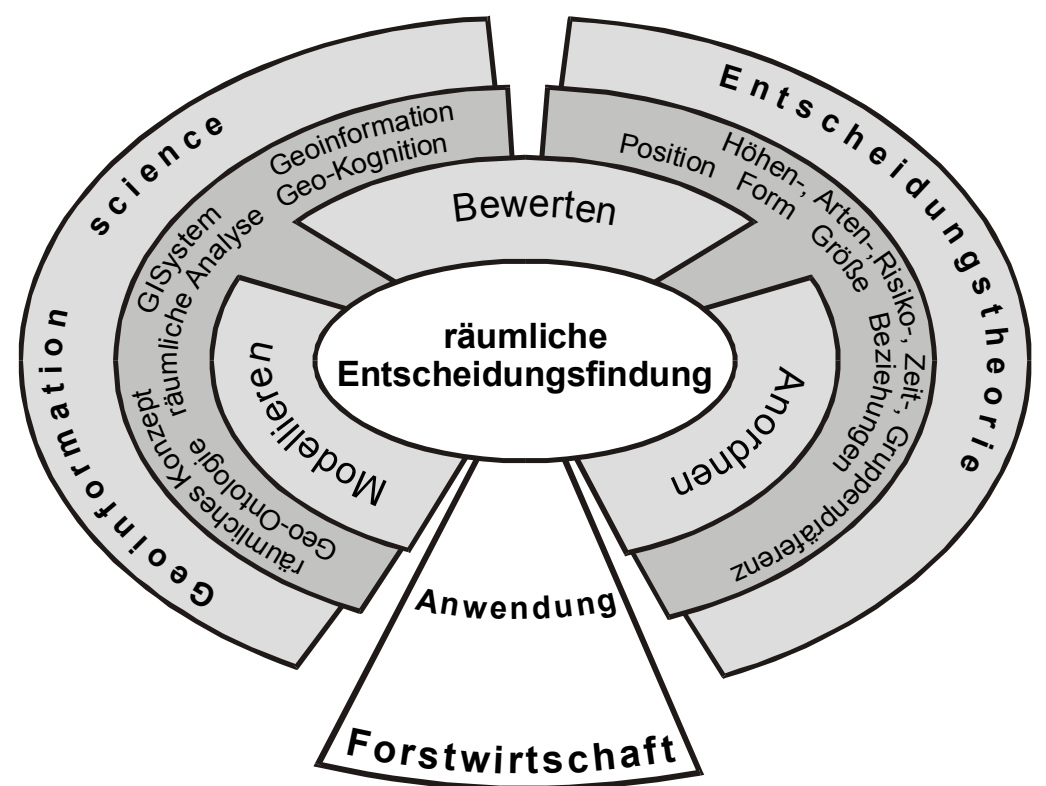

Abb. 2: Semantischer Aufbau der Arbeit.

Die Forstwirtschaft dient in dieser Arbeit als Anwendungsbereich, an dessen Beispiel Struktur und Aufbau räumlicher Entscheidungsprobleme, sowie die Prozesse ihrer Lösung dargestellt werden sollen. Damit soll der in Zukunft steigenden Bedeutung von Entscheidungs- und Planungsmethoden Rechnung getragen werden.

Das ZIEL dieser Arbeit ist die Untersuchung der positionsbezogenen Konzepte von räumlichen Objekten, die Darstellung einer Konzeption der räumlichen Entscheidungsfindung und die Untersuchung ihrer Annahmen und Hintergründe. Am Beispiel einer forstlichen Fragestellung soll der Umgang mit Geoinformationen zur Lösung räumlicher Entscheidungsprobleme aufgezeigt werden.

Die Arbeit ist entsprechend der Abbildung 1 in drei Themenbereiche gegliedert: GIScience/GISsystem, Entscheidungstheorie und forstlicher Anwendungsbereich (siehe auch Abbildung 2 und 3). 
Im Kapitel 2 wird das forstliche Planungs- und Entscheidungsmodell dargestellt und die Motivation räumlicher Entscheidungsfindung in der Forstwirtschaft wird erläutert.

In den Kapiteln 3-4 werden Geoinformation Science (GIScience) und Geoinformationssystem (GISsystem) vorgestellt. Es wird auf die wachsende Bedeutung der Geoinformation eingegangen und zugrundeliegende geographische Konzepte werden behandelt, welche für die Entscheidungsfindung von Bedeutung sein können.

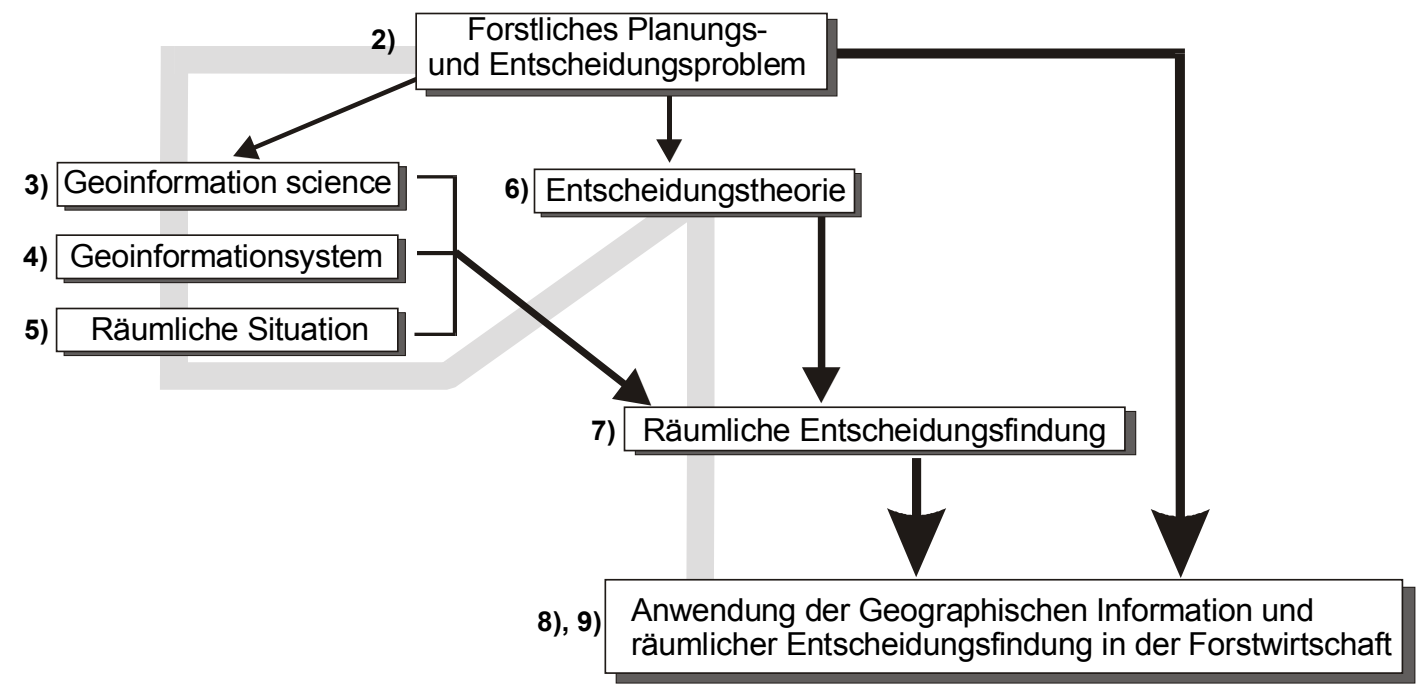

Abb. 3: Aufbau der Arbeit nach Kapiteln geordnet.

Kapitel 5 behandelt die räumliche Situation. Die Komponenten eines räumlichen Objektes (Geoobjektes) sowie Methoden ihrer aspektweisen Beschreibung werden näher betrachtet. Besondere Aufmerksamkeit gilt dabei den positionsbezogenen Informationen räumlicher Objekte. Auch auf die Theorie der räumlichen Beziehungen wird ausführlich eingegangen.

Im Kapitel 6 wird kurz die Entscheidungstheorie, vor allem aber ihr Präferenzsystem und Axiome, vorgestellt. Anschließend werden multikriterielle Entscheidungsmethoden behandelt, die sich in komplexen räumlichen Entscheidungssituationen als besonders geeignet erwiesen haben.

Im Kapitel 7 befassen wir uns mit der räumlichen Entscheidungssituation und -findung. Die Struktur räumlicher Entscheidungssituationen wird zuerst anhand der räumlichen Situation behandelt. Es wird auch auf die räumliche Präferenz und ihre Abbildungsmöglichkeiten eingegangen. Die räumlichen Entscheidungsprobleme werden nach den einer Entscheidung zugrundeliegenden Komponenten gegliedert.

In Kapitel 8 werden die Beiträge der GIScience für die Forstwirtschaft diskutiert. Dabei werden für einige Handlungssituationen in der Forstwirtschaft die räumliche Situation beschrieben, Konzepte formuliert und Entscheidungsansätze dargestellt.

Im Kapitel 9 wird räumliche Entscheidungsfindung am Beispiel der Abgrenzung von Harvestereinsatzorten im Betriebsbezirk Lichtenhagen dargestellt. 
Aus der formalen Sicht ist die Arbeit folgendermaßen aufgebaut (s. Abbildung 4). Das Kapitel 2 beschreibt Problembereich, Notwendigkeit sowie Motivation der räumlichen Entscheidungsfindung in der Forstwirtschaft. Die Kapitel 3-6 beschreiben den Hintergrund der Lösungsansätze, aspektweise Zerlegung des Problems sowie Möglichkeiten seiner Lösung. Kapitel 7 stellt methodische Werkzeuge zur Anwendung im Problembereich vor. Das Kapitel 8 befasst sich schließlich mit den Möglichkeiten der Anwendung dieser Werkzeuge anhand praktischer Beispiele.

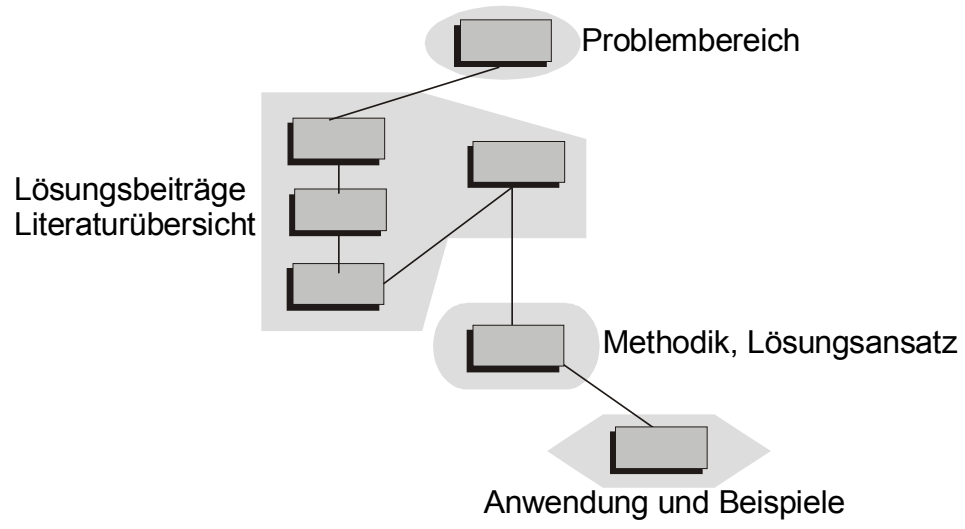

Abb. 4: Formale Gliederung der Arbeit. 


\section{Forstliches Planungs- und Entscheidungsmodell}

\subsection{Forstliches Planungsmodell}

Der Wald ist schon wegen seiner Ausdehnung, ca. 30\% der Landoberfläche der Erde, eines der wichtigsten landschaftsbildenden Elemente. Die sich aus der Nutzung des Waldes ergebenden Konsequenzen und Auswirkungen gehen über die Waldgrenze hinaus und beeinflussen sowohl die umliegenden Landschaftselemente in Nachbarschaft, als auch die globale Umwelt und die Lebensbedingungen auf der Erde. Forstwirtschaft ist eine Form der Bodennutzung, die in Mitteleuropa den Landschaftscharakter unseres Lebensraumes prägt und natürliche Grundlagen menschlichen Daseins wesentlich beeinflusst (SPEIDEL 1972).

Die Forstwirtschaft strebt eine planmäßige und zielbezogene Nutzung von Produkten des Waldes an. Die Regelung dieser Nutzung setzt eine zweckgebundene dynamische Abbildung der Realität in einem Planungsmodell voraus, in dem alle relevanten Eigenschaften und strukturellen Beziehungen der Realität erhalten bleiben und durch die Regelungskomponenten ergänzt werden. Die der Realität zugrundeliegenden natürlichen und die durch den Menschen in Kraft gesetzten Prozesse vereinigen sich in einer Veränderung der Realität, die wiederum in dem Planungsmodell abgebildet wird. Die Planung soll das Wirtschaften effektiver und effizienter regeln: Sie soll Übersicht in komplexen Situationen schaffen, das Wichtige von dem Nebensächlichen zu unterscheiden ermöglichen, das Risiko von Fehlentscheidungen verringern und helfen, bei unvorhergesehenen Ereignissen, rasch und gezielt $\mathrm{zu}$ handeln (BACHMANN et al. 1996). „Die Gestaltung eines Produktionssystems bezweckt, die einzelnen Prozessschritte räumlich und zeitlich so anzuordnen und aufeinander abzustimmen, dass der gesamte Produktionsprozess technisch wirksam, ökonomisch und ökologisch effizient sowie für Umwelt und Mensch verträglich ist" (HEINIMANN 1998).

Die Forstwirtschaft als zielgerichtete planmäßige Handlung ist über zweihundert Jahre alt. Während dieser Zeit wurde eine Vielzahl forstlicher Verfahren als Bestandteil des jeweiligen Paradigmas entwickelt. Paradigmen können nach KUHN (1997) als eine Konstellation, eine Menge von Überzeugungen, Wertvorstellungen und Techniken definiert werden, die alle (oder zumindest die überwiegende Mehrheit der) Mitglieder eines bestimmten Bereiches akzeptiert haben. Sie beschreiben eine Menge von Theorien, Standards und Methoden, die gemeinsam einen Weg repräsentieren, Wissen zu organisieren (KUHN 1997). Paradigmen enthalten nicht nur explizite wie implizite Feststellungen über die Beschaffenheit von Natur und Wirklichkeit und die Entitäten, die darin zu finden sind, sie definieren auch den zulässigen Problembereich, legen akzeptable Methoden zu seiner Erschließung fest und setzen Qualitätskriterien für die Lösungen.

Ein Paradigmawechsel wird vor allem durch die Änderung der Sicht- und Denkweise verursacht, die nicht selten durch technologische Weiterentwicklung oder Theorienentwicklung eingeleitet wurde. In der Forstwirtschaft haben die Unterschiede in der wirtschaftlichen Zielsetzung, Differenzierung des Waldaufbaues und der Betriebsarten und unterschiedliche Sicherheiten der zur Verfügung stehenden Informationen zum Paradigmawechsel der Waldregelung beigetragen (SPEIDEL 1972). Ein Spezifikum der Forstwirtschaft, das sich aus den langen Produktionszeiten 
ergibt, ist die Verflechtung unterschiedlicher Paradigmen, welche in dem bestehenden Waldaufbau wiederzufinden sind. „Die Kenntnis der historischen Verfahren ist für den Planer deswegen von Bedeutung, weil die gegenwärtigen Methoden der Forsteinrichtung noch zahlreiche Bestandteile alter Verfahren enthalten und weil der gegenwärtige Waldaufbau in Anbetracht der langen Produktionsdauer durch die Anwendung historischer Planungsverfahren geprägt wurde“ (SPEIDEL 1972). Die vorzufindenden Waldstrukturen entsprechen einer Kombination der regelmäßig angewendeten Waldbausysteme, die auch lange nach dem Ablösen eines Paradigmas wiederzufinden sind.

Die frühe Waldbewirtschaftung hatte die durch zunehmende Entwaldung verursachten Naturkatastrophen und die Holznot zu bekämpfen. Die wirtschaftliche Umwelt dieser Zeit war noch ziemlich einfach, vorwiegend auf das Produkt Holz war das Interesse der Gesellschaft ausgerichtet. In der weiteren Entwicklung hat sich die Situation angesichts der fortschreitenden Industrialisierung und der damit verbundenen Gesellschaftsumwandlung geändert. Die Forstwirtschaft ist unter massiven Kostendruck geraten. Mit zunehmender Industrialisierung hat sich das Verhältnis der Holzpreise zu Produktionskosten zu Ungunsten des Forstbetriebes verändert, was durch Verknappung von öffentlichen Geldern und damit verbundener immer geringerer Bereitschaft zur Förderung noch verstärkt wurde. Die Gesellschaft formulierte zusätzliche Anforderungen an den Wald und das Waldökosystem: die Schutz- und Erholungsfunktionen des Waldes gewannen an Bedeutung. In der weiteren Entwicklung ist die Forstwirtschaft in immer stärkeren Umbruch geraten, weil sich die Sichtweise und damit verbundene Ziele und Aufgaben geändert haben. Der ökologisch-orientierte Waldbau oder der naturnahe Wirtschaftswald (BITTER 1998A) ist das neue Paradigma der Forstwirtschaft, wodurch sowohl Naturnähe als auch das Wirtschaften betont werden. Gleichzeitige Verbesserung von ökologischer Stabilität als auch wirtschaftlicher Leistungsfähigkeit wird angestrebt.

Die mittlerweile fortgeschrittene Durchsetzung partizipativer Planungs- und Entscheidungsvorgehensweisen in der Raumplanung lässt vermuten, dass die Partizipation auch in der forstlichen Planung künftig angewendet wird. Partizipation bezeichnet im allgemeinen die Teilnahme an politischen und sozialen Entscheidungsprozessen. In der Schweiz heißt es, die Waldregelung soll in Zukunft den Wald als Ganzes, unabhängig von Eigentumsgrenzen, betrachten. Die übergeordneten Ziele der Walderhaltung und -entwicklung sollen in einer gesamträumlichen Planung festgehalten werden (BACHMANN et al. 1996). Mit der überbetrieblichen Planung geht das schweizerische Bundeswaldgesetz über den üblichen Rahmen der Forstplanung hinaus und fügt die Pflicht zur Information und Mitwirkung der Bevölkerung ein. Die überbetriebliche Planung soll dabei die Planung auf der Ebene des Forstbetriebes, bzw. Waldeigentümers ergänzen, um so die forstlichen Anliegen in die Raumplanung oder in andere Sachplanungen einzubringen (BACHMANN et al. 1996). In Kanada wurden die "public-policy issues" in der Forstwirtschaft von der Bevölkerung als Element der Entscheidungsfindung breit akzeptiert: „Productive public involvement in forest decision-making can, on one hand, prevent unnecessary conflict, and, on the other, channel existing and new conflicts into collaborative searches for accommodating solutions" (ANONYMUS 1998C).

Die aktuelle Situation der forstlichen Planung ist durch zunehmend strukturreicher werdende Wälder, die Fortentwicklung der Inventurtechnik und die fortschreitende Entwicklung der Datenverarbeitung gekennzeichnet (KOCH \& SMALTSCHINSKI 1997). Der Anforderungskatalog an die Planung ist wesentlich größer geworden. Genaue und sichere Informationen über Zustand, Entwicklung und Funktionen der Wälder sollen eine genügend genaue und ausreichend flexible 
Planung ermöglichen, die auch den Anforderungen der übergeordneten Planungen genügen und den betriebswirtschaftlichen Verhältnissen entsprechen muss (BöCKMANN et al. 1998b). „Mit Bewunderung betrachten wir heute das ganzheitliche Denken unserer forstlichen Klassiker, da wir selbst in der Informationsflut zu versinken drohen und die Zusammenhänge der einzelnen Fakten nicht mehr erkennen" (SPELLMANN 1991).

Neue Aufgaben der Forstwirtschaft verlangen neue Verfahren zur Umsetzung der Leitbilder sowie zur Inventur, Planung und Kontrolle. Die Methoden der Forsteinrichtung sollen laufend an die Veränderung der Anforderungen des forstlichen Planungsmodells angepasst werden (BÖCKMANN at al. 1998B). Den vielfältigen Aufgaben der forstlichen Planung und Bewirtschaftung kann nur durch ein gezieltes Informationsmanagement Rechnung getragen werden. Das forstliche Informationssystem hat dabei primär mit räumlichen Daten, Operationen, analytischen und entscheidungstechnischen Methoden zu tun. Die Entscheidungsunterstützung soll in der heutigen Flut an Informationen den Entscheidungsträger entlasten, ihm die Entscheidungen erleichtern und sie transparent machen. Bei zunehmender Anzahl der an einer Entscheidung beteiligten Personen soll das Informationssystem zur Entscheidungsunterstützung auch eine Gruppenzusammenarbeit ermöglichen, in deren Rahmen die gemeinsamen Ziele und alternativen Handlungsmöglichkeiten formuliert werden können. Auf starre allgemeingültige Richtlinien und Vorgehensweisen wird verzichtet. Kreativität ist angesagt: Die Vorgaben verlieren an bindender Wirkung, betriebsindividuelle Lösungen werden gefordert, betrieblicher Gestaltungsfreiraum soll auch in öffentlichen Verwaltungen ausgeweitet werden und die Verantwortlichkeit vor Ort wird sich erhöhen (BITTER 1998A).

\subsection{Entscheidungsfindung in der Forstwirtschaft}

Die Regelung des Waldes, während der die vielfältigen Entscheidungen in eine Ordnung gebracht werden, richtet sich nach dem zu erfüllenden Zielsystem des Forstbetriebes. In der mehrfunktionellen Forstwirtschaft, die durch zunehmende äußere Einflüsse und das Einbeziehen von betriebsexternen Zielen in den Regelungsprozess gekennzeichnet ist, steigt das Bedürfnis zielbewusster Alternativenwahl eines rationalen und effektiven Entscheidens. Die Vielfalt der Ansprüche an den Nutzen des Ökosystems Wald mündet in ein Bündel sich gegenseitig beeinflussender Entscheidungen mit mehrfacher Zielsetzung und mit breiterer Beteiligung. Die Ziele in einer solchen Situation stehen in Konkurrenz teils bis hin zum gegenseitigen Ausschluss.

Die Entscheidungsfindung in der Forstwirtschaft ist wegen der vielseitigen Interdependenzen äußerst komplex. Die spezifischen Eigenschaften forstlicher Entscheidungen sind die Unsicherheit bezüglich der weiteren Entwicklung und eine strukturelle Abhängigkeit individueller Entscheidungen. Die Interdependenzen betreffen sowohl die räumliche als auch die zeitliche Auswirkung der Alternativen. „Beim Objektsystem, Wald' handelt es sich um ein hochkomplexes Ökosystem, über das nur unvollständige und unsichere Informationen über Ursache/Wirkungsbeziehungen sowie Wechselwirkungen zwischen einzelnen Faktoren des Systems bekannt sind“" (VACIK 1999).

In einem Forstbetrieb werden täglich Entscheidungen getroffen. Die klassische, auf die Holzproduktion ausgerichtete Waldregelung umfasst von der Gründung eines Bestandes über 
seine Pflege und Vornutzung bis hin $\mathrm{zu}$ seiner Endnutzung eine Fülle von assoziierten Entscheidungen. Diese betreffen die Wahl der Verjüngungsart und Baumartenzusammensetzung, Zeitpunkt, Stärke und Art der Pflege, die Endnutzungsart und Weise, den Transport des Holzes, die Reihenfolge der zu erfüllenden Waldfunktionen usw. Bei der heutigen Situation der immer stärkeren Verflechtung der forstlichen mit „forst-fremden“ Paradigmen (Naturschutz, Landschaftspflege) kommen zusätzliche Entscheidungen meistens mit ausgeprägtem Raumbezug hinzu.

Die Komplexität der Entscheidungssituationen führt dazu, dass in vielen Fällen eine imperativsubjektive Entscheidungsweise überwiegend ist. Vor allem die räumlichen waldbaulichen und forsteinrichtungstechnischen Entscheidungen werden als Expertenentscheidungen mit eingeschränkter Transparenz getroffen. Die Bestände sind bezüglich der Standortbedingungen und des Bestandesklimas sowie anderer Bedingungen derart komplex, dass die Erfassung aller zur Entscheidung notwendigen Informationen sehr aufwendig ist und gegenüber einer Expertenentscheidung als weniger effizient erscheint. Auch die Wiederverwendbarkeiteit der Informationen ist umstritten, da sich die realen Verhältnisse so ändern können, dass die wiederholte Aufnahme unerlässlich ist. Jedoch ist eine transparente Darstellung der Entscheidung durch die Modellierung der Entscheidungssituation und Abbildung der zielbezogenen Präferenzen für den Planungsprozess eine unerlässliche Aufgabe.

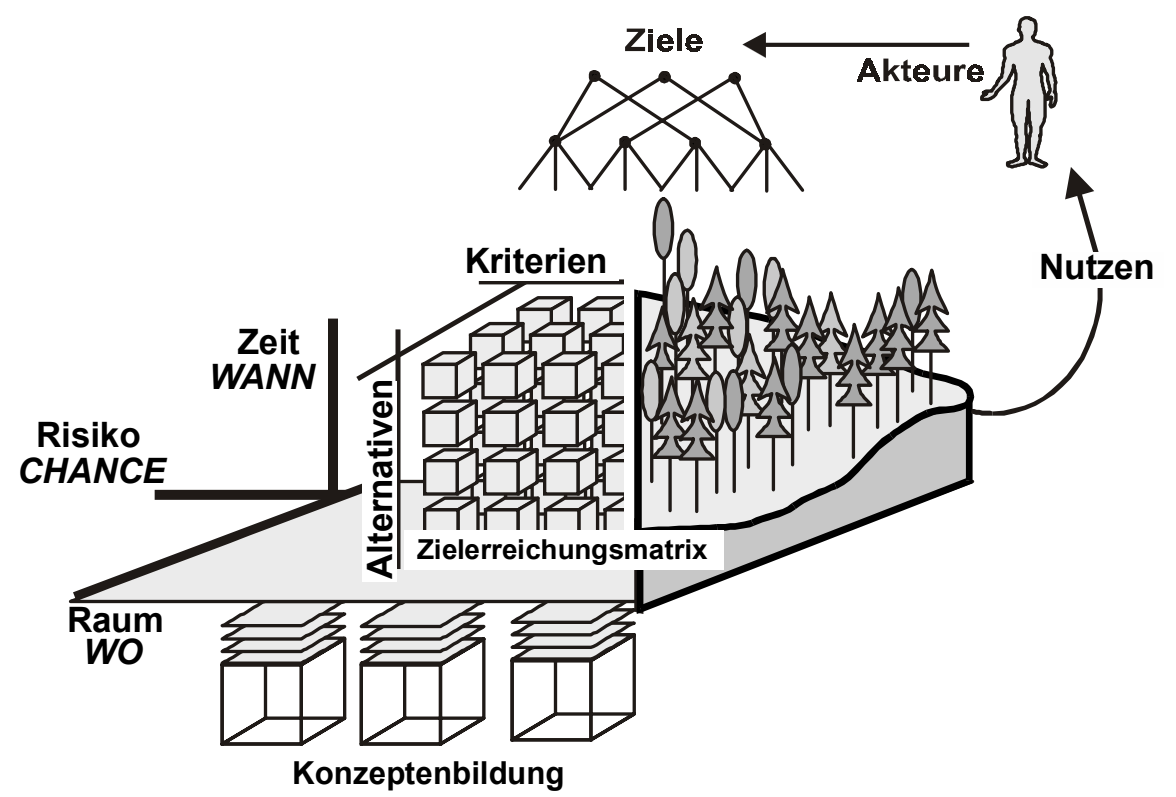

Abb. 5: Schematische Darstellung forstlicher Entscheidungen.

Die schematische Darstellung des forstlichen Entscheidungssystems wird in Abbildung 5 gezeigt: Jede räumliche Einheit des forstlichen Planungsmodells ist eine Variable des Regelungssystems, ihre Handlungsmöglichkeiten sollen zur Erfüllung des gesamten Zielsystems eines Entscheidungsträgers beitragen. Durch ihre räumliche Komponente (Form, Größe, Ausrichtung und Position) prägt sie dem Gegenstand der Regelung (dem Waldbesitz, der Landschaft) eine räumliche Struktur ein. Durch die unterschiedliche Position der räumlichen Einheiten in der Hierarchie des forstlichen Planungsmodells ergibt sich ein hierarchisch aufgebautes System von sich gegenseitig beeinflussenden räumlichen Entscheidungen, durch deren Zusammensetzung die bestmögliche Erfüllung der aufgestellten Ziele erzielt werden soll. 


\subsection{Räumliche Entscheidungen in der Forstwirtschaft}

Die Mehrheit der Entscheidungen in der Forstwirtschaft hat einen räumlichen Bezug oder eine räumliche Auswirkung. Die forstliche Planung, als kreativer Prozess der Entscheidungsfindung (SCHMIDT 1998), versteht sich als raum-zeitliche Planung. Sind der angestrebte Nutzen oder mit ihm verbundene Fragen räumlich differenziert, d.h. besitzen die Ziele des Entscheidungsträgers räumliche Aspekte, so haben auch die zugrundeliegenden Entscheidungen einen räumlichen Charakter.

Die räumliche Entscheidungsfindung kann in der Forstwirtschaft vor allem in der Planung räumlicher Ordnung Anwendung finden. Räumlicher Ordnung wurde die vorrangige Aufgabe zugewiesen, das Produktionsrisiko abzugrenzen und $\mathrm{zu}$ verteilen. „Das Ziel der räumlichen Ordnung besteht darin, durch Aufbau und geographische Lagerung der Bestände sowie durch Ausnutzung und Schaffung natürlicher und künstlicher Schutzvorrichtungen eine optimale Produktionssicherheit zu erreichen“ (SPEIDEL 1972, S.140). Darüber hinaus spielt sie eine aktive Rolle bei der Frage der Nachhaltigkeit und der damit verbundenen räumlichen und zeitlichen Verteilung der Nutzung.

Tab. 1: Beispiele der Bereiche, in welchen räumliche Entscheidungsfindung in der Forstwirtschaft traditionell Bedeutung hat.

\begin{tabular}{|c|c|c|}
\hline \multicolumn{2}{|c|}{ Entscheidungsproblem } & Beschreibung des Ziels \\
\hline \multirow{3}{*}{ 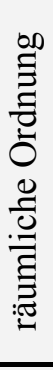 } & Waldeinteilung & $\begin{array}{l}\text { Die Wirtschafts- und Planungseinheiten so abzugrenzen, dass die } \\
\text { Maßnahmen während der Produktionszeit möglichst homogen sind. } \\
\text { Das Abgrenzungskriterium sind } \quad \text { Bestockung oder } \\
\text { Standortverhältnisse. }\end{array}$ \\
\hline & Waldaufbau & $\begin{array}{l}\text { Das Neben- und Übereinander der Bäume und Baumkollektive zu } \\
\text { Gunsten der Sicherheit und maximaler Produktion zu gestalten. }\end{array}$ \\
\hline & Walderschließung & $\begin{array}{l}\text { Die Waldeinheiten für Personen, Güter und Maschinen zugänglich } \\
\text { machen. }\end{array}$ \\
\hline & $\begin{array}{l}\text { besta } \\
\text { Ziels }\end{array}$ & $\begin{array}{l}\text { Die Festlegung der vorrangigen oder der Rangfolge der von einem } \\
\text { Bestand zu erfüllenden Aufgaben (Funktionen) }\end{array}$ \\
\hline & Technologieeinsatz & Anwendung technologischer Mittel in der Produktionskette „,Holz“ \\
\hline & $\begin{array}{l}\text { raum-zeitliche } \\
\text { Verteilung }\end{array}$ & $\begin{array}{l}\text { Aus unterschiedlichen Gründen (Nachhaltigkeit, Diversität) } \\
\text { angestrebte räumliche Verteilung der Baumkollektive oder ihrer } \\
\text { Eigenschaften. }\end{array}$ \\
\hline
\end{tabular}

Die Schaffung einer räumlichen Ordnung des Waldes ist eine vorrangige Aufgabe der Waldregelung. Die Waldeinteilung, die Erschließung und der Waldaufbau tragen alle (im unterschiedlichen Ausmaß) zur räumlichen Gestaltung des Waldes bei. Alle drei Bereiche sind weitgehend untrennbar, wovon sich Konsequenzen bei der Lösung entsprechender Entscheidungsprobleme ergeben. Nach KURTH et al. (1994) sind die Aufgaben räumlicher Ordnung:

(A) den Wald für Inventur, Planung, Vollzug und Kontrolle einzuteilen,

(B) günstige ökologische Bedingungen für das Wachstum zu schaffen,

(C) günstige Voraussetzungen für technologische Prozesse herzustellen,

(D) Schäden zu mindern. 
Die Waldeinteilung, -erschließung und -aufbau tragen in unterschiedlicher Weise zur Erfüllung dieser Aufgaben bei. Die Waldeinteilung dient vor allem der ersten Aufgabe, dabei sind jedoch auch die anderen drei Aufgaben zu berücksichtigen. Die Walderschließung hat das Ziel, den Wald für Mensch, Technologie und Material zugänglich $\mathrm{zu}$ machen, die Erschließungseinrichtungen werden aber oft auch als Bestandesgrenzen gewählt und stehen dann mit der Waldeinteilung in einem Interdependenzverband. Der Waldaufbau strebt günstige ökologische Bedingungen für die Produktion und gleichzeitig die Schadensminderung an.

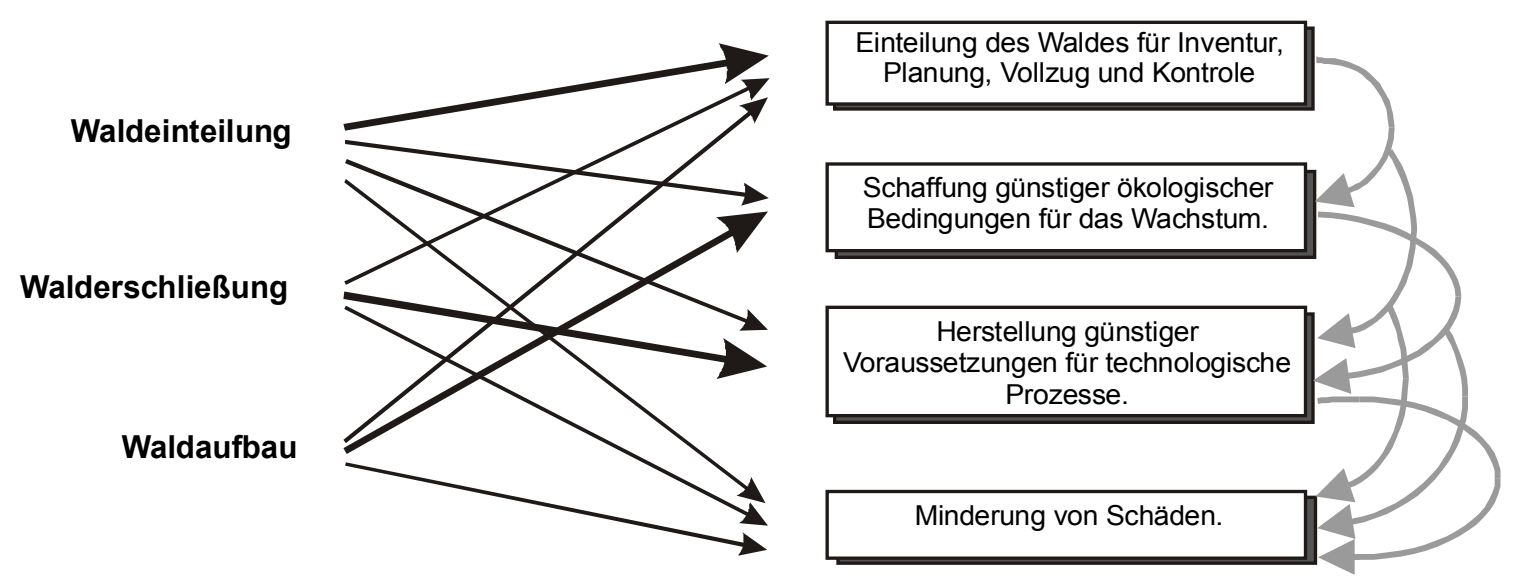

Abb. 6: Interdependenzen der räumlichen Ordnung (Inhalt der Graphik nach KURTH et al. 1994).

Die Waldeinteilung ist heute vor allem durch die angestrebte Kontinuität gekennzeichnet. Die Abteilungs- und Unterabteilungsbildung kommt heute nur bei Neuaufforstungen vor. Die Basiserschließung durch Wege ist in Mitteleuropa ebenfalls abgeschlossen. Das vorhandene Wegenetz wird nur an die veränderten Bedingungen angepasst. In der naturnahen Bewirtschaftung gewinnt die Plenterordnung zunehmend an Bedeutung. Bei immer strukturreicher werdenden Beständen und verschwindenden Bestandesübergängen verliert auch der Waldaufbau an ursprünglicher Bedeutung.

Diese Tatsachen prägen das zugrundeliegende Entscheidungsproblem: Wegen vielfältiger Interdependenzen ist die Schaffung günstiger räumlicher Ordnung das komplexeste Ziel der Forstwirtschaft. Sie kann als eine Vielzahl assoziierter Entscheidungsprobleme mit unterschiedlicher räumlicher und zeitlicher Auswirkung und Skala betrachtet werden. Die Entscheidung einer Entscheidungssituation schränkt die Handlungsmöglichkeit von anderen ein.

Die Ansätze und Modelle der räumlichen Ordnung sind durch starre Leitbilder gekennzeichnet. Der Grund dafür liegt vor allem in dem Aufwand einer solchen Entscheidungsfindung, deren effektive Bearbeitung erst in den letzten Jahren ermöglicht wurde. Da schon am Anfang der forstwirtschaftlichen Regelung der Waldentwicklung Entscheidungen über die räumliche Ordnung getroffen werden mussten, hat man sie vor allem mit Hilfe mentaler Entscheidungsfähigkeiten der Forstleute bewältigen müssen. Heutige landschaftlich-ökologische Fragestellungen stellen neue Ansprüche an die räumliche Ordnung.

Die Entscheidung über den Einsatz der Technologie ist ein alltägliches Problem in der Forstwirtschaft. Mechanisierte Ausführung entlastet die Arbeiter, was bei der schweren physischen Belastung bei der Waldarbeit sehr willkommen ist. Außerdem kann eine bessere 
Effektivität und dadurch ein ökonomischer Vorteil erzielt werden. Die mechanisierte Arbeitsausführung ist der manuellen in der Forstwirtschaft daher grundsätzlich vorzuziehen, wo es die geographischen Bedingungen ermöglichen und wo dies nicht zu einer Beeinträchtigung anderer Vorhaben bzw. Ziele führt.

Bei den Entscheidungen über Technologieeinsatz trifft man auf eine Zusammensetzung verschiedenartiger Kriterien und $\mathrm{zu}$ berücksichtigender Faktoren. Die Naturbedingungen, beschrieben durch lokale Geomorphologie und Boden, sowie die Bestandeseigenschaften bezüglich der Baumartenzusammensetzung und der Bestandesstruktur schränken die Einsatzmöglichkeiten im wesentlichen ein und lassen nur einen relativ kleinen Entscheidungsraum. Das vorhandene Erschließungsnetz ist ebenfalls bei der Entscheidung über den Einsatz einer Technologie von Bedeutung. Hier wird deutlich, dass die Konsequenzen dieser Entscheidungen, wie es in der Forstwirtschaft die Regel ist, von den Konsequenzen anderer bereits getroffener Entscheidungen abhängen, was zu vielfältigen Interdependenzen führt.

Das Entscheidungsproblem kann als Abgrenzung der geeigneten Flächen für den Einsatz oder als Bestimmung der Art der Arbeitsausführung für jede Fläche des Waldgebietes bestimmt werden. Im ersten Fall wird einer Arbeitsausführung die geeignete Fläche zugeordnet, im zweiten Fall wird jeder Fläche eine Arbeitsausführung zugeordnet. Die Komplexität dieser Entscheidungssituation lässt sich beliebig steigern: So kann z.B. nicht nur eine Entscheidung bezüglich des Fällens der Bäume, sondern gleichzeitig auch bezüglich ihrer Bringung getroffen werden. Auch der optimale Zeitpunkt des Einsatzes kann in Betracht gezogen werden.

Das Entscheidungsproblem über den Einsatz der Technologie wird zu einem räumlichen Entscheidungsproblem, wenn die von dem Entscheidungsträger verfolgten Ziele Räumlichkeit enthalten. Dies zeichnet sich dadurch aus, dass der Entscheidungsträger räumliche Kriterien zur Operationalisierung der Ziele anwendet. Diese können beispielsweise als Hangneigung, Bodenbelastbarkeit, Anteil an der Bestandfläche, Entfernung zu den Wegen, Konzentration der geeigneten Flächen, etc. formuliert werden.

Bei dem Holzrücken werden die Bäume von dem Stock (Fällungsort) zum Platz des Lagerns transportiert. Die erste Entscheidung dabei betrifft das Rückemittel. Welches Rückemittel angewendet werden kann, entscheidet vor allem die Geländegeomorphologie. Die Zugänglichkeit eines Bestandes kann als Ausschlusskriterium betrachtet werden. Darüber hinaus können weitere Kriterien angewendet werden, welche die Wirtschaftlichkeit oder die ökologische Auswirkung alternativer Rückemittel miteinander abwägen können. Die anschließenden Entscheidungen werden am Beispiel eines Rückeschleppers beschrieben (Lehnhausen 1982).

Der Schlepperfahrer fährt mit dem Schlepper zum Bestandesrand, von woher er sich für eine Position im Bestand entscheiden muss, welche er für geeignet hält, um eine bestimmte Anzahl der gefällten Stämme zusammenzuziehen. Nachdem er diese Position ausgewählt hat, muss er sich für eine Menge der Stämme entscheiden, an die er das Seil befestigt, um sie an den Schlepper heranzuziehen. Die stehenden Bäume stellen Hindernisse dar, welche das Rücken der Stämme verhindern bzw. erschweren können. Die von dem Schlepperfahrer verfolgten Ziele betreffen die gesamte Arbeitszeit des Rückens, der Umfang der Schäden am verbleibenden Bestand, die Anzahl der Hin- und Rückfahrten des Schleppers, etc. 
Der Schlepperfahrer muss die räumliche Situation an dem Fällungsort abschätzen können, die räumlichen Beziehungen der liegenden Stämme zueinander und zu den stehenden Bäumen und die Beziehung der ausgewählten Trasse im Bestand zu stehenden Bäumen und zu der Last beurteilen. Die Erfahrung hilft ihm dabei, eine gute Strategie zu wählen, um alle Stämme in möglichst kurzer Zeit und unter möglichst kleinem Aufwand aus dem Bestand herauszuziehen (vg. LEHNHAUSEN 1982).

Obwohl die computergestützte Entscheidungsfindung in diesem Fall eher umstritten erscheinen mag, wurde dieses Beispiel hier zur Dokumentation eines räumlich ausgeprägten Verhaltens bei der Waldbewirtschaftung gewählt.

Die Bewältigung neuartiger Aufgaben stellt erhöhte Anforderungen an die Fähigkeit der Forstleute, räumliche Konzepte $\mathrm{zu}$ formulieren und anzuwenden. Bei räumlichen Entscheidungen, wie beispielsweise bei Biotopschutz oder Reservatausweisung, werden forstfremde Konzepte zusammen mit den forstlichen (Nutzungsart, -dauer) angewendet. Die aktive Teilnahme der Forstwirtschaft an diesen Entscheidungen setzt die Formulierung eigener räumlich ausgeprägter Präferenz der Forstleute voraus, die sich auf die räumlichen Konzepte stützt.

Die Fähigkeit, räumliche Konzepte $\mathrm{zu}$ entwickeln und im Prozess der mentalen Informationsverarbeitung anzuwenden, ist eine primäre Kognitionsfähigkeit der Menschen. Die Subjektivität räumlicher Konzepte kann mit formalen Methoden beseitigt werden, um die Transparenz zu erhöhen. Die Menschen entwickeln räumliche Konzepte, die für eine angegebene Fragestellung von Bedeutung sind. Da diese im Grunde subjektiver Natur sind, ist es vor allem wegen Transparenz, Nachvollziehbarkeit und Überprüfbarkeit nötig, sie zu objektivieren, d.h. klar zu formulieren.

Die mentalen räumlichen Modelle (kognitive Karten) sind nicht zuverlässig für die Planung komplexer Sachverhalte. Sie enthalten nur bestimmte Aspekte der Räumlichkeit und nur vage Informationen diesbezüglich. Der Mensch hat bereits am Anfang seiner geographischen Erkundung einfache Abbildungen der Räumlichkeit in Form der Karten benutzt, um diese Unzulänglichkeiten seiner kognitiven Modelle zu überwinden. Im komplexen System mehrerer Ziele im Umbau des forstlichen Paradigmas scheint die computergestützte Planung ein unausweichliches Mittel zur Erreichung der angestrebten Ziele.

Die räumliche Entscheidungsfindung stellt ein Mittel dar, das für die forstliche Planung von großer Bedeutung sein könnte. Um seine Möglichkeiten jedoch nutzen zu können, muss die Räumlichkeit forstlicher Planung genauer untersucht werden. Es müssen Antworten auf folgende Fragen gefunden werden:

- Welche Art von räumlichen Entscheidungen enthält die forstliche Planung?

- Wie werden räumliche Entscheidungen bewältigt?

- Welche Präferenzen werden während der forstlichen Planung angewendet?

- Welche räumlichen Objekte und Beziehungen sind dabei von Bedeutung?

- Welche Interdependenzen bestehen zwischen den Entscheidungen? 


\subsection{Ableitung einer räumlichen Entscheidungssituation}

Um die Unterschiede einer räumlichen Entscheidungssituation von anderen Fragestellungen zu zeigen, greifen wir auf das Beispiel in SPORS et al. (1992) zurück. Es handelt sich um ein einfaches Beispiel, das die Nutzung der Geographischen Informationstechnologie (in ihrer Anfangsphase) zu erläutern beabsichtigte.

\section{Beispiel:}

Eine Landesforstverwaltung ist daran interessiert, die geeigneten Flächen für den Einsatz eines Harvesters (Eingriffs-Vollernter) für die Nadelholzdurchforstung im Durchmesserbereich 12-35 cm zu finden. Die Einschränkung des Einsatzes erfolgt durch die Bedingung der Befahrbarkeit (bis zu 30 \% Neigung) und der potentiellen Gefährdung von Waldstandorten (bodenökologische Eigenschaften).

Diese Fragestellung stellt kein Entscheidungsproblem dar. Es bestehen zwei alternative Möglichkeiten (motormanuelle und mechanisierte Arbeitsausführung), der Entscheidungsträger (Landesforstverwaltung) ist aber nicht a priori unsicher, welche Alternative gewählt werden soll. Er hat sich bereits bei der Problemformulierung entschieden, in welchen Situationen er der mechanisierbaren Arbeitsausführung den Vorzug geben wird. Die Fragestellung ist lediglich eine Anfrage an eine Geodatenbank, die zur Selektierung geeigneter Flächen führen soll. Im vorgegebenen Raum (Betriebsbezirk Lichtenhagen) sollen alle Flächen gefunden werden, die gleichzeitig einem Nadelholzrein- oder - mischbestand (Inventureinheit) mit vorgeschriebener Durchforstung (Planungseinheit), einem gering bis mäßig gefährdeten Standort (Standorteinheit) und einer räumlichen Einheit (konzeptionellem Derivat des Geländemodells) mit der Neigung bis $30 \%$ angehören.

Die Räumlichkeit in der Fragestellung umfasst die Hangneigung, die Standorteigenschaften sowie die Waldeinteilung: die Einsatzorte sollen ,innerhalb' geeigneter Bestände ,auf' geeigneten Standorten und ,auf' geeigneten Geländeformen liegen.

Die Fragestellung kann zu einer Entscheidungssituation erweitert werden, wenn sich der Entscheidungsträger für das vorgegebene Anspruchsniveau der Kriterien erst während der Entscheidungsfindung entscheidet. Um den Übergang zu einer Entscheidungssituation deutlicher zu machen, wird das Beispiel in Entscheidungsprobleme modifiziert, die durch eine zunehmende Komplexität charakterisiert sind.

Problemdesign 1: Formulierung eines einfachen Entscheidungsproblems

Die erste Modifikation umfasst die Erweiterung der Ansatzvorstellungen um das Kriterium "Anteil der Nadelholzarten" an der Artenzusammensetzung des Bestandes. Der Entscheidungsträger ist zuerst über seine Präferenz bezüglich der unteren Grenze des Mischanteils von Nadelholzarten unsicher und will sie erst während der Lösungssuche äußern.

Das einzige Kriterium zur Beurteilung der Eignung einer Fläche zum Einsatz eines Harvesters ist das Vorkommen von Nadelholzarten in der Baumartenzusammensetzung. Die gesuchte Entscheidung liegt in der Festlegung einer Mindestanforderung an den erwarteten Anteil, einer Art der Satisfaktionsbedingung (Anspruchsniveau). Diese Entscheidung will der Entscheidungsträger (ET) erst dann fällen, wenn er über die Eigenschaften der in Frage 
kommenden Flächen informiert wird. Es fällt ihm danach nicht schwer, die Entscheidung zu treffen. Der ET muss keine Aggregation verschiedener Kriterien der Zielerfüllung vornehmen, seine Aufgabe besteht in der Anordnung möglicher Ausprägungen des Kriteriums und in der Festlegung der Mindestgrenze. Danach werden alle solchen Bestände von dem Einsatz des Harvesters ausgenommen, welche die Mindestanforderung nicht erfüllen.

Kriterien

Baumartenzusammensetzung

\section{Bedingungen}

Standort

Hangneigung

Problemdesign 2: Formulierung eines mehrkriteriellen Entscheidungsproblems

Der Entscheidungsträger zeigt seine Bereitschaft, von der Strenge seines Anspruchsniveaus beim Kriterium der Standortsgefährdung geringfügig abzusehen, wenn die geeignete Fläche, nicht sehr groß' ist oder einen, erheblichen Anteil' der Fläche des zugehörigen Bestandes ausmacht. Außerdem will er strenger die Hangneigung beurteilen, wobei in dem Spielraum von 20-30\% der Hangneigung nur solche Flächen vollmechanisiert durchforstet werden sollen, die entsprechend ,groß` sind und bei denen die Gefährdung ,möglichst gering' gehalten werden kann.

Die in Frage kommenden Flächen werden in bezug auf 5 Kriterien beurteilt, die bei den einzelnen Alternativen nicht gleichmäßig gute Ausprägungen annehmen. Dabei gilt, dass eine schlechte Ausprägung in einem Kriterium durch eine bessere Ausprägung in einem anderen Kriterium begrenzt ausgeglichen werden kann. Die Entscheidung besteht darin, die Präferenz des Entscheidungsträgers bezüglich der Kompensation abzufragen und einzelne Kriterien miteinander zu kombinieren.

\section{Kriterien}

Baumartenzusammensetzung

Standortsgefährdung

Fläche der Alternativen

Anteil der Alternativen an der

Bestandesfläche

Hangneigung

\section{Bedingungen}

Standort

Hangneigung

Fläche der Alternativen

Anteil der Alternativen an der

Bestandesfläche

\section{Problemdesign 3: Formulierung eines räumlichen Entscheidungsproblems}

Der Entscheidungsträger will bei seiner Entscheidung ein zusätzliches Kriterium verwenden, welches die Anordnung der geeigneten Flächen zueinander betrifft. Der Entscheidungsträger ist daran interessiert, den Harvestereinsatz nur bei größeren Konzentrationen von Holzmasse anzuordnen, damit auch die Transportkosten des Harvesters gering bleiben. Bei einer engeren räumlichen Konzentration (räumliches Muster) der Flächen wird der Harvester auch bei größeren Flächen mit weniger geeignetem Standort und kleineren Anteilen an Nadelholzarten der manuellen Durchforstung vorgezogen.

Das Design dieser Entscheidungssituation bringt neue Elemente in unser Beispiel. In den vorherigen Beispielen war die Räumlichkeit der Alternativen nicht von Bedeutung, die Entscheidung konnte nur anhand der individuellen Eigenschaften der Alternativen getroffen werden. In diesem Design kommt es auch auf die Distanz der Bestände zueinander an. Der Entscheidungsträger besitzt eine räumliche Präferenz über die Alternativen. Er ist bereit, von 
einer schlechteren Ausprägung bei anderen Kriterien abzusehen, wenn die Alternative eine günstige relative Position besitzt.

Kriterien
Baumartenzusammensetzung
Standortsgefährdung
Fläche der Alternativen
Anteil der Alternativen an der Bestandesfläche
Hangneigung
räumliche Konzentration der geeigneten
Flächen

\author{
Bedingungen \\ Standort \\ Hangneigung \\ Fläche der Alternativen \\ Anteil der Alternativen an der \\ Bestandesfläche
}

Problemdesign 4: Formulierung eines raum-zeitlichen Entscheidungsproblems

In der letzten Modifikation besitzt der Entscheidungsträger auch solche Ziele, welche die Alternativen um eine zeitliche Komponente erweitern. Der Entscheidungsträger will den Harvester in einem Bestand während der gesamten Produktionsdauer nur einmal einsetzen, um den Boden zu schonen. Die Alternativen umfassen in diesem Design nicht nur die Flächen mit den geeigneten Eigenschaften, sondern auch den Zeitpunkt des Einsatzes des Harvesters. Die Kriterien dieses Entscheidungsdesigns unterscheiden sich nicht von denen des Designs 3. Wegen des impliziten Zeitbezugs ist der Gegenstand der Entscheidung die dynamische räumliche Lage der Alternativen.

\subsection{Zusammenfassung der Motivation und des Vorhabens der Arbeit}

Die räumliche Entscheidungsfindung kommt immer dann zum Einsatz, wenn ein räumliches Entscheidungsproblem vorliegt, d.h. wenn die alternativen Handlungsmöglichkeiten eines Entscheidungsproblems anhand ihrer räumlichen Konsequenzen beurteilt und angeordnet werden sollen. Die Ziele des Entscheidungsträgers können in einer solchen Situation weiter nach räumlichen Aspekten strukturiert werden, der Raum dient als Strukturierungskonzept.

Die räumlichen Aspekte können in der Forstwirtschaft in verschiedenen Zielen enthalten sein. Das Ziel an sich muss nicht offensichtlich Räumlichkeit enthalten: Zum Beispiel ist die Diversität eines naturnahen Waldbaues ein nicht-räumliches (a-räumliches) Ziel, solange sie nur größtmögliche Anzahl der Baumarten angestrebt. Sie wird jedoch auch zu einem räumlichen Ziel, wenn sie ebenfalls auch die räumliche Verteilung der Arten berücksichtigt. Ähnlich ist die Nachhaltigkeit, das leitende Prinzip der forstlichen Regelung, a-räumlich (nicht-räumlich), wenn sie nur einen nachhaltigen Vorrat (sprich Menge) des Holzes anstrebt, den größtmöglichen Zuwachs und die Beständigkeit der Nutzung anstrebt. Wird jedoch auch eine räumliche Verteilung des Vorrats oder der Waldgesellschaften darunter verstanden, so enthält das verfolgte Ziel bereits Räumlichkeit in sich. Stabilität als Ziel der räumlichen Ordnung ist bereits ein räumliches Ziel, wohingegen die Stabilität im bezug auf die standortgerechte Baumartenzusammensetzung die Räumlichkeit nicht implizit enthält.

Räumliche Entscheidungsfindung kann in komplexen räumlichen Entscheidungssituationen dabei helfen, rasch eine rationale und transparente Entscheidung zu finden. In der Forstwirtschaft kann 
ihr eine wichtige Rolle zukommen, da wegen verschiedenen Nutzenskonflikten die Räumlichkeit immer größere Bedeutung hat.

Diese Arbeit befasst sich mit der Räumlichkeit und den Konsequenzen ihrer Anwendung in der Forstwirtschaft. Es wird gezeigt, wie die Räumlichkeit formuliert und formalisiert werden kann, wie sie für den Prozess der Entscheidungsfindung strukturiert wird und wie diesbezüglich Präferenz geäußert werden kann. 


\section{Geoinformation science}

\subsection{Raum und Räumlichkeit}

Raum und Zeit in ihren Relationen sind die grundlegenden, allgemeinen Existenzformen sich bewegender Materie (KURTH et al. 1994). Raum ist ein grundsätzliches Strukturierungskonzept, das die Trennung zwischen Individuum und Umwelt erlaubt (ROTHER 1998). Je nach Betrachtungsweise nimmt der Raum unterschiedliche Formen an. Es wird zwischen einem objektiven, im Sinne eines abstrakten (mathematischen) oder konkreten (physischen, geographischen) Raumes, und einem subjektiven (auch relativ genannt) individuell erlebten Raum unterschieden (SPITZER 1991). Der objektive Raum zeichnet sich entweder durch die Abwesenheit oder vielfältige Unendlichkeit der Auszeichnungen und Bedeutungen aus. Im geographischen Raum ergibt die Bestimmung des menschlichen Daseins durch den Raum, das Zurechtfinden und sich Einrichten im Raum die Räumlichkeit der Existenz (SPITZER 1991). "Die Räumlichkeit und die Zeitlichkeit unseres Körpers bilden die Gitterstäbe eines Käfigs, in dessen Grenzen sich unsere Existenz entfaltet" (WEICHHART 1998).

Der Raum dient als gemeinsame Basis der Objekte oder Phänomene. Die in bezug auf räumliche Dimensionen konkret festgelegte, geometrisch oder geodätisch fixierbare Position im Raum wird als absolute Lage (Position, location) bezeichnet. Die relative Position bezeichnet den Kontext einer Lage im Verhältnis zu ihrer Umgebung (WEICHHART 1998). Die Räumlichkeiten werden allgemein als Lagerelationen verstanden.

Der objektive mathematische Raum kann als eine Hierarchie der Raumtypen beschrieben werden, die durch Transformationsgruppen festgelegt sind. Geometrie befasst sich mit der Untersuchung der Eigenschaften der geometrischen Figuren oder einer Anordnung (Konfiguration) der geometrischen Figuren, die bei einer bestimmten Gruppe der Transformationen unverändert (invariant) bleiben (vgl. BITTNER 1998). Den Grundstein einer Klassifizierung der Geometrie hat KLEIN (1872, zitiert nach BITTNER 1998) mit seinem sog. 'Erlanger Programm' gelegt. Das Klassifizierungsprinzip basiert auf dem Automorphismus des Raumes. Das Erlangerprogramm fasst Geometrie als Invariantentheorie einer Abbildungs- bzw. Transformationsgruppe auf, die auf einem Trägerraum operiert (BOHNE 1995). Die Transformationsgruppe definiert in einem geometrischen Raum eine Äquivalenzrelation in der Gesamtheit der Figuren und damit Klassen zueinander äquivalenter Figuren bzw. invarianter Eigenschaften.

Der geographische Raum kann als partielle Ordnung subjektiver räumlicher Beobachtungen mit unterschiedlicher Skalierung und Auflösung gesehen werden. Der Raum wird von einem Zentrum aus (hier: Position des Beobachters) beobachtet. Der geographische Raum ist ein großmaßstäblicher Raum (large-scale space). Er kann aus einer Position nicht ganz erforscht werden. Er enthält Objekte, die viel größer als der menschliche Körper sind, und die nicht manipuliert werden können. Die Erforschung dieses Raumes ist partiell und setzt Bewegung voraus.

Die räumlichen Objekte und Phänomene werden durch ihre Position (absolut, relativ), Semantik (Attribute), Dynamik, Relationen und Skalen der Beobachtung beschrieben. Semantik und 
Position sind grundlegende Aspekte räumlicher Phänomene. Die Position, bestimmt durch WO (where), bildet mit der Semantik, WAS (what), eine untrennbare Einheit. Die Dynamik charakterisiert die zeitlichen Veränderungen sowohl der thematischen als auch der positionsbezogenen Eigenschaften. Die relative Position wird mit räumlichen Beziehungen (Relationen) ausgedrückt. Zwei grundlegende Beziehungsarten des geographischen Raumes sind Distanz und Richtung. Diese Beziehungen werden bei den räumlich ausgedehnten geographischen Objekten, die einen Körper besitzen, um eine weitere, im Gegensatz zu den ersten zwei Beziehungsarten eine primär qualitative Beziehung, die Topologie, erweitert. Die Anwendung der Hierarchisierung als ein Strukturierungskonzept in der räumlichen Domäne führt zu räumlichen hierarchischen Beziehungen.

Der räumliche Körper räumlich ausgedehnter Objekte kann durch Form, Größe und Ausrichtung beschrieben werden. Die Änderung der Eigenschaften kontinuierlicher räumlicher Phänomene (Felder, fields) ist von Position zu Position im Raum stetig. Die menschliche Konzeptualisierung solcher Phänomene versucht sie zu diskretisieren: Dies geschieht entweder durch die Ausscheidung von Objekten oder durch die Partitionierung des Raumes.

Die Analyse und Modellierung räumlicher Verteilungen und Prozesse (spatial analysis, spatial modeling) ist eine grundlegende Aufgabe aller Geowissenschaften. Räumliche Theorien versuchen, die Eigenschaften der Phänomene zu erklären, die durch ihre absolute oder relative Lage bestimmt sind (WEICHHART 1998). Die Verteilung der Objekte und Phänomene im Raum, charakterisiert durch Dichte, Konzentration und Muster sowie räumliche Assoziation, räumliche Autokorrelation und räumliche Interaktion, sind dabei von zentraler Bedeutung.

\subsection{Geoinformation}

Die durch das Wahrnehmen, Einschätzen oder Vermessen eines geographischen Phänomens entstandene Information wird als Geoinformation (GI) bezeichnet. Die GI kann als Information "about the features and phenomena located in the vicinity of the surface of the Earth" (GOODCHILD et al. 1999) definiert werden. Was sie von anderen Informationen unterscheidet, ist ihr Bezug zu einer geographischen Position (Raumbezug). "All GI can be reduced eventually to a simple statement that at some location there exist an instance of some more generally recognized thing, where things might be a class, a feature, a concept, a measurement of some variable, an activity, an organism, or any of a myriad possibilities" (GOODCHILD et al. 1999). Andere Definitionen der GI betonen gelegentlich ihre Aspekte. GOODCHILD (1997) spricht beispielsweise von "knowledge about where something is, knowledge about what is at a given location", CLINTON spricht von Informationen, die "identifies the geographical location and characteristics of natural or constructed features and boundaries on the earth" (ANONYMUS 1994A).

Geoinformationen unterscheiden sich von anderen (a-räumlichen) Informationen durch einige Eigenschaften, die auch im weiteren ihre Handhabung und Verarbeitung prägen (ANSELIN 1989, OPENSHAW 1996, BuRROUGH 1992, ANONYMUS 1996B): Geoinformationen entstehen als Approximation unendlicher Erdoberfläche: sie beschreiben die räumlichen Phänomene nur auf bestimmter Skala oder Auflösung oder nur für einen bestimmten Moment in der Zeit.

Geodaten beschreiben mehrere Fälle (Objekte/Punkte) und räumlich autokorrelierte Variablen.

Die Verteilungen der Geodaten unterscheiden sich von den klassischen statistischen 
Verteilungen. Die Fehler sind sehr komplex, meistens nicht zufällig und können auch räumlich strukturiert werden. Die Maßstabs- und Aggregationseffekte (modifiable areal unit) erschweren die räumliche Analyse.

Die Geoinformationen zeichnen sich außerdem durch ihre universale Wichtigkeit für viele Aktivitäten moderner Gesellschaft sowie ihre Interdisziplinarität aus.

\subsection{Politische, wirtschaftliche und wissenschaftliche Dimensionen der Geoinformation}

Die Bedeutung der GI nimmt in vielen Bereichen der Forschung und des praktischen Lebens zu. Sie ist zu einem "Wirtschaftsgut ersten Ranges" geworden (ANONYMUS 1998A). In ANONYMUS (1998A) wurde die Bedeutung der GI für die Gesellschaft kurz so charakterisiert: "Geoinformationen bilden einen wesentlichen Teil des in der modernen Informations- und Kommunikationsgesellschaft vorhandenen Wissens. Sie werden auf allen Ebenen in Verwaltung, Wirtschaft, Wissenschaft und vom Bürger benötigt; sie sind Grundlage des planerischen Handelns und ihre Verfügbarkeit maßgebliche Voraussetzung für Standort- und Investitionsentscheidungen".

Der Geodatenmarkt betrifft rund die Hälfte aller Wirtschaftszweige. Der europaweite Umsatz für hochwertige Geoinformationen wurde im "GI-2000"-Papier der EU-Kommission für 1996 mit 550 Mio. ECU angegeben, wobei mit einer 14\%igen jährlichen Steigerungsrate zu rechnen sei. Hinzu kommen Systemausgaben für Hard- und Software (vgl. ANONYMUS 1998B). Im 1998 wurden die Investitionen für die Erstellung und Nutzung der GI in Europa auf $10 \mathrm{Mrd}$. Ecu im Jahr geschätzt (DG XIII/E - August 1998). Der Umsatz des weltweiten GIS Software- und Dienstleistungsmarktes näherte sich 19994 Mrd. \$ (USA Firmen mehr als die Hälfte). Der Markt mit GIS Software, Dienstleistungen und Daten umfasste 1998 in USA 4.2 Mrd. \$ (MARK 1999A).

Nach den Umsatz- und Wachstumsprognosen der Europäischen Kommission DGXIII (19971998) beträgt im Bereich der GI das zu erwartende Anwachsen des Marktes 140\% bis zum Jahre 2001. Die Schätzungen aus den USA sprachen noch optimistischer von exponentiellem Wachstum.

Mitte der 90er Jahre wurde in mehreren Ländern der wachsenden Bedeutung der GI auch mit sog. Nationalen Raumdateninfrastrukturen Rechnung getragen. In CLINTONS ORDER wird diese als "technology, policies, standards, and human resources necessary to acquire, process, store, distribute, and improve utilization of geospatial data" definiert (ANONYMUS 1994).

Die in den 90er Jahren entstandenen internationalen und nationalen Verbände im Bereich der Geoinformation übernehmen die Interessenvertretung: EUROGI (European Umbrelle Organisation for Geographic Information) auf europäischer Ebene, der Deutsche Dachverband für Geoinformation DDGI, Österreichischer Dachverband für Geographische Information AGEO, The Association for Geographic Information AGI (UK), Schweizerische Organisation für Geo-Information SOGI, und weitere.

GI 2000 ist eine Initiative der Generaldirektion Informationsgesellschaft DG XIII EK im Rahmen des INFO 2000 Programms, die sich die Unterstützung gemeinsamer europaweiter GI Politik 
zum Ziel gesetzt hat. Das Ziel dieser Aktivität war die Bildung eines "policy framework... for a stable, European-wide set of agreed standards, procedures, guidelines and incentives for creating, updating, changing, accessing and using geographic information" (ANONYMUS 1999D).

Im Bereich der Forschung wurde die Bedeutung der GI erkannt und die Weiterentwicklung ihrer theoretischen Grundlagen angestrebt. In den USA entstand in den späten 80er Jahren das NCGIA (National Center for Geographic Information and Analysis). Bei ihrer Entstehung hat die National Science Foundation (und andere GIS Kommunitäten in den USA) die GI und die geographische Analyse als "critical areas for research" (ANONYMUS 1999A) anerkannt. 1994 haben 34 US Universitäten das University Consortium for Geographic Information Science (UCGIS) gegründet, dessen Ziel "the development and use of theories, methods, technology, and data for undestanding geographic processes, relationship, and pattern" ist (MARK 1999A).

Die erste europäische GIS Konferenz fand 1990 in Amsterdam statt. Das 1993 gestartete Programm GISDATA der European Science Foundation hatte das Ziel, die existierenden nationalen Forschungsaktivitäten zu erweitern und eine Zusammenarbeit bei GI Integration, Datenbankdesign und sozialen und umweltwissenschaftlichen Anwendungen zu unterstützen. Am Ende des 4-jährigen Programms hatten insgesamt 300 Forscher aus 20 Ländern an dieser Aktivität teilgenommen. Die interdisziplinäre Konferenz COSIT (Conference on Spatial Information Theory) fand 1993 zum ersten Mal statt. Ihr Thema umfasst die theoretischen Aspekte des Raumes und der geographischen Information (large scale space information), ihre Repräsentation und Verarbeitung.

1998 wurde AGILE (Association of Geographic Information Laboratories in Europe) mit dem Ziel begründet, die akademischen Lehr- und Forschungsaktivitäten im GIS Bereich auf europäischer Ebene zu unterstützen. Dabei werden die Aktivitäten fortgesetzt, die schon im Rahmen des Programms GISDATA und der EGIS Konferenzen entstanden sind. Außerdem soll eine europäische Forschungsagenda geführt werden. Die 1992 gegründete Geographical Information Systems International Group (GISIG) beabsichtigt eine Kooperation von GIS Nutzern durch Unterstützung gemeinsamer europäischer Projekte im Bereich der GIS Ausbildung, -Forschung und -Entwicklung.

Als ein vorrangiges Ziel im GI Bereich wurde die Interoperabilität der GIS angestrebt. 1994 wurde durch eine Vereinigung von Anwendern und Anbietern aus dem öffentlichen und privaten Bereich das OpenGIS Consortium (OGC) ins Leben gerufen. Das Ziel des Konsortiums ist es, ein allgemeines Konzept (Spezifikation) für die Implementierung offener GIS festzulegen, die den freien Austausch von Operationen und Daten möglich machen. In Europa wurde 1998 GISPIE (GIS Interoperability Project Stimulating the Industry in Europe) gestartet. Das Ziel dieses Projekts war, die europäischen GI-Unternehmen und Forschungsinstitutionen zur engeren Zusammenarbeit bei der Spezifikation offener Geoinformationstechnologien (OpenGIS) zu bringen und dadurch die Konkurrenzfähigkeit der europäischen GI-Industrie zu verbessern (ANONYMUS 2000C). 


\subsection{Geographische Kognition}

Geographische Information entsteht durch (mentale) Fähigkeiten des Menschen, die räumlichen Konzepte aus der geographischen Realität zu abstrahieren, sie in geeigneter Form zu repräsentieren, zu speichern und zu verarbeiten.

Die Kognition (lat. cognoscere: erkennen, wahrnehmen, wissen) folgt der Aufnahme und Verarbeitung von sensorischen Reizen. Das Aufgenommene wird mit Bekanntem (Wissen) verglichen und aus dem Vergleich werden Schlüsse gezogen. Das Wissen muss erworben (Lernprozess), gespeichert (Repräsentation im Gedächtnis) und aufgerufen (Suche) werden.

Die Kognition (als psychologischer Prozess) steht zwischen der Wahrnehmung und dem Handeln. Sie umfasst Wahrnehmen, Gedächtnis, Lernen, Denken, Problemlösen, Gebrauch der Sprache, motorische Steuerung u.a.: "Cognition includes sensation and perception, thinking, imagery, reasoning and problem-solving, memory, learning, and language. Cognitive structures and process are part of the mind, which emerges from a brain and nervous system inside of a body that exists in a social and physical world" (MONTELlo 1997). Die kognitiven Prozesse sind die Grundlage für intelligentes Verhalten (STRUBE 1996).

Die geographische Kognition (geospatial, spatial, geographical cognition) bezeichnet die Kognition in der räumlichen und speziell in der geographischen Domäne. Forschung im Bereich der geographischen Kognition befasst sich mit Wahrnehmung, Gedächtnis, Handlung und Kommunikation der raum-zeitlichen und zeitlichen Eigenschaften der Objekte und Ereignisse in realer oder digitaler Repräsentation (vgl. ANONYMUS 2000B). Von Interesse sind hier die räumlichen Eigenschaften wie Position, Größe, Entfernung, Richtung, Form, Muster, Bewegung und Beziehungen (MONTELLO 1997).

Das Wissen über den geographischen Raum unterscheidet sich von anderen Arten des Wissens. Der Raum wird über verschiedene Kanäle direkt wahrgenommen, die unterschiedliche Modalitäten wiedergeben (ZIMMERMAN \& FREKSA 1993). Das Wissen, das durch einen Kanal aufgenommen wird, kann durch andere bestätigt oder widerlegt werden. Die wahrgenommene Welt besitzt eine Positionsperspektive, sie wird von einem Punkt (Position des Beobachters) unkomplett beobachtet. Die erworbene Information ist redundant, konstant und betrifft nur die wichtigen Objekte und Ereignisse.

Räumliche Kognition befasst sich vor allem mit folgenden Themen (MARK et al. 1999B):

- Erwerb des geographischen Wissens (acquisition of geographical knowledge)

- Mentale Repräsentation des geographischen Wissens (mental representation of geographical knowledge)

- Verwendung des geographischen Wissens (geographical knowledge use)

- Kommunikation (Austausch) der geographischen Information (communication of geographical information)

Der Maßstab des Raumes spielt in der räumlichen Kognition eine wichtige Rolle. In einem kleinmaßstäblichen Raum (small-scale space) sind die Objekte kleiner als der menschliche Körper und können bewegt werden. Im Gegensatz dazu sind die Objekte in einem 
großmaßstäblichen oder geographischen Raum (large-scale space) größer als der Mensch und dieser kann sich zwischen ihnen bewegen. In der Kognitionswissenschaft wurden bezüglich des Maßstabes bzw. der Größenordnung mehrere Raumtypisierungen entwickelt. Aus ihrem Vergleich ergeben sich drei gemeinsame Eigenschaften aller Raummodelle: Handhabbarkeit (manipulability), Bewegung (locomotion), Größe (size) (FREUNDSCHUH \& EGENHOFER 1997).

Der Erwerb des Wissens über den geographischen Raum setzt eine Bewegung durch ihn voraus, während welcher komplexe Information extrahiert wird. Die sensormotorische Information wird in ein geographisches Wissen konvertiert, wobei die Umweltinformationen zur Generierung von räumlichen Eigenschaften benutzt werden (MONTELLO 1997). Die traditionelle Theorie der Entwicklungssequenzen (developmental sequence) des räumlichen Wissens (nach der Piaget'schen Theorie) enthält 3 Stadien des Wissens, das mit der Zeit gewonnen wird. Zuerst wird das "landmark knowledge" entwickelt, ein eindeutiges Muster von visuellen, eine Position einnehmenden Gegenständen. Durch die Verbindung geordneter Sequenzen mit keinen oder wenigen metrischen Informationen wird danach ein "route knowledge" entwickelt. Im weiteren entwickelt sich ein "survey knowledge", ein zweidimensionales Anordnungswissen, das neben anderen auch eine Umleitung, Abkürzung und kreative Navigation erlaubt.

Die prinzipielle Frage der Kognition ist die Repräsentation des Wissens. Bei der Frage der Repräsentation und der Verarbeitung des räumlichen Wissens werden die propositionalen und bildhaften (analogen) Ansätze formuliert. Die bildhaften Ansätze gehen über eine rein propositionale Repräsentation hinaus und behaupten die Existenz eines piktoriellen (bildhaften) Kodes der Informationsverarbeitung, des sog. mentalen Bildes (PRIBBENOW 1993). Mentale Modelle sind schematisch, bis zu einem gewissen Grad unvollständig und nicht streng logisch aufgebaut. Eine klare Bestimmung des Begriffes "mentales Modell" gibt es aber nicht. Sie stellen individuelle Denkmodelle dar, die das Verständnis eines Sachverhalts prägen, "mit deren Hilfe wir planen und entscheiden, vorausschauen und erklären, kurz: mit deren Hilfe wir denken" (HASEBROOK 1995).

Mentale Raumrepräsentationen sind nicht beständig, (geographischen) Karten ähnlich. Sie sind für einen bestimmten Zweck aus relevanten Informationen (eventuell aus unterschiedlichen Quellen) konstruiert. Die Raumvorstellung eines Menschen wird in einer kognitiven Karte (mental, cognitive map) festgelegt, die ein Relativraumkonzept repräsentiert. Als Maßeinheiten von dieser können relative Distanzmaße wie Kosten, Zeitaufwand, Güteraustauschvolumen oder Anzahl sozialer Kontakte angewendet werden. Die Menschen entwickeln (und erleben) gleichzeitig eine Vielzahl von Relativräumen. Die kognitiven Karten werden in der Form des kognitiven Atlas (cognitive atlas) als eine Kollektion der kognitiven Karten mit unterschiedlichen Skalen verwaltet. Angesichts der Multimedialität, Fragmentalität und Partialität der mentalen Raumvorstellungen wird die Sammlung der individuellen Repräsentationen als kognitive Collage bezeichnet (TVERSKY 1993).

Die ,Naive Geographie' (EGENHOFER \& MARK 1997) versucht, das Alltagsverständnis (commonsense) mit formalen Modellen $\mathrm{zu}$ beschreiben. Diese sollten in künftige GIS implementiert werden, um dem allgemeinen geographischen Verständnis entgegenzukommen.

Die Forschung im Bereich der Geokognition ist von Bedeutung u.a. im Bereich von Datengewinnung und Speicherung, graphischer Repräsentation, Design der Benutzeroberfläche, räumlicher Analyse, Interoperabilität, Entscheidungsfindung und sozialem Kontext der GIS 
(ANONYMus 1996B). Die Nutzung, Effizienz und Rentabilität der Geographischen Informationssysteme können durch diese Forschung verbessert werden. Aus der Sicht der räumlichen Entscheidungsfindung sind vor allem folgende Themen der Geokognition von Interesse:

- Wie entwickeln Menschen Konzepte vom Raum und wie handeln sie anhand dieser? Wie beeinflusst Training und Erfahrung diese Fähigkeiten?

- Wie benutzen und verstehen die Menschen Sprache über Raum, und über Objekte und Ereignisse im Raum?

- Wie kann komplexe GI dargestellt werden, um Verständnis und Entscheidungsfindung zu unterstützen?

- Wie unterscheiden sich die subjektiven (alters-, geschlechts- oder kulturbedingten) kognitiven Fähigkeiten der Menschen und warum?

- Wie kann die Entwicklung neuer geographischer Informationstechnologien die Weise ändern, wie die Menschen den Raum wahrnehmen und über ihn denken ?

\subsection{Räumliche Konzepte}

Ein räumliches Konzept ist eine informale oder formale Beschreibung des menschlichen Verstehens des Raumes, der Objekte im Raum und der Beziehungen zwischen den Objekten (SCHNEIDER, M. 1997). Es umfasst alle Ansichten, welche die räumlichen Aspekte einer Untermenge der realen Welt beschreiben.

A)

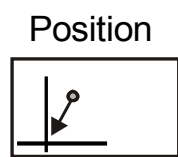

B)
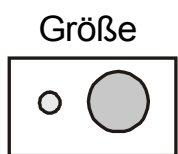

Nachbarschaft Verteilung

C)
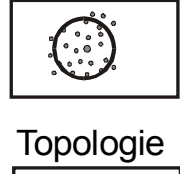

D)

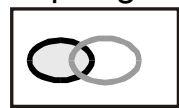

Koordinaten

Referenzsys. Skala

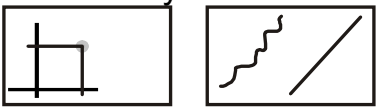

Ausrichtung
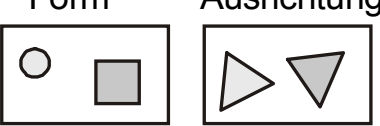

Muster
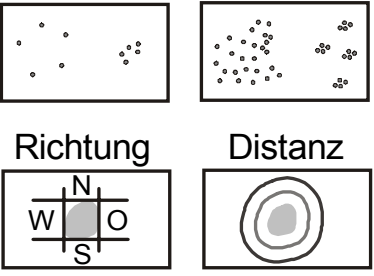

Abb. 7: Beispiele räumlicher Konzepte: A) Konzepte der Abbildung von Geoinformationen; B) individuelle objektbezogene Konzepte; C) Konzepte der räumlichen Verteilung; D) individuelle räumliche Beziehungen.

Alle Methoden, die zur Organisation und Wahrnehmung des Raumes dienen, beschreiben das räumliche Konzept (Abbildung 7). Beispiele solcher Konzepte können sein (GoODCHILD et al. 1999):

- Konzepte, die die Basis der GIS beschreiben (Referenzsystem, Metrik).

- Allgemeine Klasse der 'Dinge' (things), die das Phänomen mit räumlicher Position definieren. 
- Begriffe und Metaphern zur Definition der Beziehung zwischen den geographischen (räumlichen) Punktmengen.

Im Prozess der computergestützten Verarbeitung geographischer Informationen müssen räumliche Konzepte in ein Computermodell abgebildet werden. Bei Operationalisierung der geographischen Konzepte hierzu sind vor allem folgende Fragen zu klären (EGENHOFER 1996; GOODCHILD et al. 1999) :

- Wie soll die geographische Welt konzeptualisiert werden?

- Wie ist die geographische Welt einer Gesellschaft (society) konstruiert und wie beeinflusst die Gesellschaft unser Verständnis der geographischen Welt?

- Wie sollen die geographischen Konzepte gespeichert werden ?

- Wie sollen die geographischen Konzepte repräsentiert werden ?

- Wie sollen die geographischen Konzepte verwaltet und transformiert werden?

- Wie sollen die geographischen Phänomene erklärt und analysiert werden ?

- Wie kann die geographische Informationstechnologie bei der Aufdeckung helfen ?

- Wie sollen die geographischen Konzepte visualisiert werden ?

- Wie sollen die geographischen Konzepte benutzt werden?

Man unterscheidet zwischen zwei komplementären Konzepten der geographischen Phänomene: das (geographische) Objektmodell (entity model oder entity view) und das Modell kontinuierlicher Änderung (field Modell oder location/occurrence).

In entity view besteht der Raum aus definierbaren und abgrenzbaren individuellen Objekten. Jedes Objekt bezieht sich auf den Raum und hat eigene Eigenschaften. Ein Objekt kann wiederum aus anderen Objekten bestehen. Die Grenzen der Objekte können oft nur unklar angegeben oder gemessen werden. Die Eigenschaften innerhalb der Grenze eines Objektes sind homogen. Dagegen besitzt ein Phänomen in der Field Repräsentation keine Grenze. Jedes seiner Attribute ist kontinuierlich und glatt. Jedem Punkt des Raumes können die Attribute zugeordnet werden.

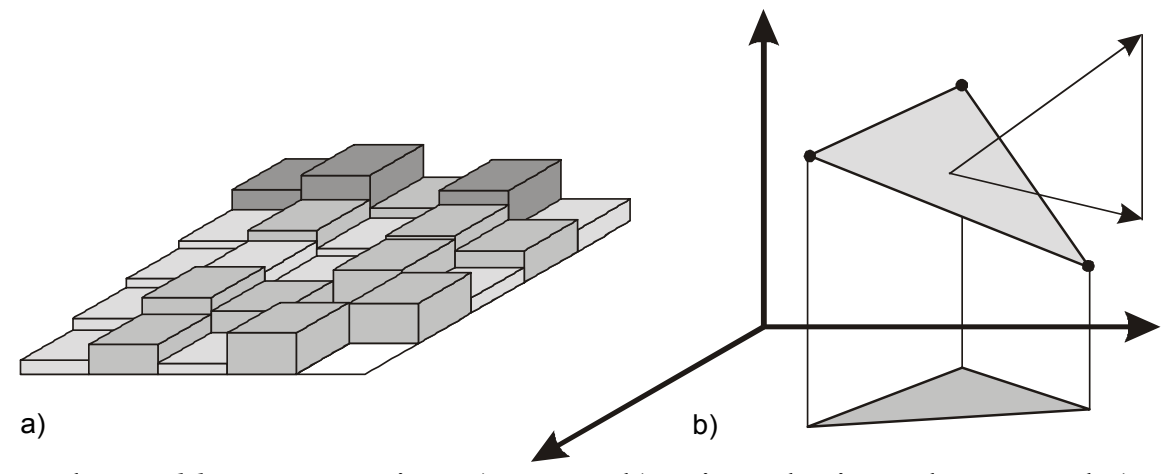

Abb. 8: Konzepte der Field-Repräsentation: a) Raster, b) Triangular irregular network (TIN).

Ein Field impliziert eine Funktion, die jedem Punkt im Raum einen Wert zuordnet $f(x, y)=a$. Ein Field ist dann eine unendliche Menge der Datensätze $\langle p$, al,a2 ..., an>, wobei $p$ die Position eines Punktes im Raum (x,y) und $a_{1} \ldots a_{n}$ alle Attribute bezeichnet, die mit diesem Punkt assoziiert sind. (GoOdCHILD 1992). Ein Field kann durch eine mathematische Funktion, eine Menge diskreter Isolinien oder approximativ durch reguläre Diskretisierung (Abbildung 8) beschrieben werden. 
Beide Abstraktionen der Realität stellen Extreme dar, beide sind wegen ihrer logischen Konsistenz und Einfachheit der Implementierung und Handhabung beliebt. Die Objekt versus Field Diskussion ist mit den Raummodellen small-scale space und large-scale space der räumlichen Kognition unmittelbar verbunden. Das Objektmodell beschreibt die Objekte aus der Sicht der small-scale space Erfahrung. Das Fieldmodell repräsentiert eher die Erfahrung von large-scale space.

\subsection{Ontologie}

Ontologien sind Theorien zur Beschreibung von Objekten, Klassen, Eigenschaften und Funktionen in einer Sichtweise der realen Welt mit formalen Methoden. Sie versuchen, der menschlichen Wahrnehmung ähnlich, die reale Welt in Klassen, Relationen und Funktionen aufzuteilen. Die philosophische Definition der Ontologie ist die Lehre vom Seienden, die formale und materielle Prinzipien des Gegebenen untersucht (ANONYMUs 2000a). Für den Bereich der künstlichen Intelligenz wurde ihre Definition konkretisiert. Unter Ontologie versteht man eine Spezifikation eines Konzeptes. "An ontology is an explicit specification of a conceptualisation" (GRUBER 1993 zitiert nach SMITH \& MARK 1998). Während in der Philosophie ihre Fragestellung als 'Was existiert' formuliert wird, ist man in der Informatik an 'Was wird repräsentiert' interessiert (vgl. EsCHENBACH et al. 1999).

Die Ontologie dient so der Abbildung eines Teiles der realen Welt im Rechner bzw. in einer Datenbank: "Ontology is the science of objects" (FRANK 1997). Sie beschreibt, was in der modellierten Welt vorkommt: die Kategorien, Identitäten und Beziehungen (KUHN 2000). Der formale Charakter der Ontologie ermöglicht, durch eine eindeutige Festlegung der Bedeutung elementarer Modellierungsprimitive die Ontologien zur Wiederverwendung von Wissensmodellen einzusetzen. Ontologie stellt einen systematischen Rahmen für interdisziplinäre Kommunikation und Zusammenarbeit zwischen unterschiedlichen Domänen der Wissenschaft dar: „The formal description of ontology is thus essential to data exchange standards, and to the design of human-computer interface" (SMITH \& MARK 1998).

Die Ontologie der geographischen Domäne (Geospatial Ontology (MARK 2000), Geo-ontologies (EGENHOFER 2000)) beabsichtigt ein besseres Verständnis der Struktur der geographischen Welt. Die grundlegenden Fragestellungen räumlicher Repräsentation betreffen Typ und Ausdehnung räumlicher Objekte, seine Bestandteile sowie Raumtheorien (CASETI et al. 1998). „Complete ontology of geospatial phenomena will perform for theoretical and applied geographic information science a similar role to that which human genome will provide for human biological, medical science, and the pharmaceuticals industry" (MARK 2000) .

Bei der Konstruktion einer Ontologie in der geographischen Domäne treten spezifische Probleme auf: Der Klassifikation liegt ein Konzept zugrunde, wonach jedes beliebige Element einer Klasse die Klasse gleich gut repräsentieren kann. Die Klasse geographischer Objekte enthält im Gegensatz dazu Objekte, die sie in unterschiedlichem Maß charakterisieren. Die geographischen Objekte sind zumeist komplex, zusammengesetzt aus einfacheren Objekten. Eine geeignete geographische Ontologie muss diese Zusammensetzung berücksichtigen. Geographische Objekte haben Grenzen, die in der Ontologie aufgenommen werden müssen. 
Ein ontology-driven Geographisches Informationssystem (ODGIS) integriert die Ontologie als eine Komponente (ähnlich der Datenbank), die mit anderen zusammenarbeitet.

\subsection{GIScience}

Der Begriff "Geographic information science" scheint sich in den letzten Jahren als akzeptable Bezeichnung für die fundamentalen Probleme der effektiven Erhebung, Interpretation, Speicherung, Analyse und Kommunikation der geographischen Information durchzusetzen (vgl. ANONYMUS 1996B).

GIScience ist als die Wissenschaft hinter der (geographischen Informations-) Technologie zu verstehen, deren Forschungsfeld sich aus der Nutzung von Systemen und Technologie ergibt (GOODCHILD 1997). Die Grundlagenforschung dieser Wissenschaft besteht aus der Re-Definition der geographischen Konzepte und ihrer Anwendung im Kontext von GIS. GIScience untersucht auch die Einflüsse von GIS auf Individuen und Gruppen und den Einfluss der Gruppe (Gesellschaft) auf GIS (MARK 1999A).

Weitere englische Bezeichnungen dieser Wissenschaft wie Geomatics, Geoinformatics, Spatial Information Science, Spatial Informations Theory werden gelegentlich präferiert. Der Begriff Spatial information theory wurde als Titel einer der bedeutensten Konferenzen im GIScience Umfeld gewählt, der COSIT Konferenz (Conference on Spatial Informations Theory). Nach ihrer Interpretation: "Spatial information theory also deals with the description of objects, processes or events in spatial environments and forms the basis for the construction of Geographic Information Systems (GIS) and for spatial information and communication system design in general" (ANONYMUS 1999C). Im deutschsprachigen Raum scheint dem Begriff Geoinformatik die Durchsetzung gelungen zu sein.

Die GIScience wird im VARENIUS ${ }^{2}$ Projekt in drei Sichten gegliedert: Wissenschaftliche (scientific), technologische (technological) und gesellschaftliche (societal) Sicht. Dieser Gliederung folgend ergeben sich drei Ebene der GIScience:

- Ebene des Individuums als Benutzer der Technologie, als Beobachter des geographischen Phänomens, als Quelle der Konzeptualisierung und als Entscheidungsträger.

- Ebene des Systems als gesamter Komplex der digitalen GI-Technologien und ihrer Unterstützung durch Software, Hardware und Netzwerke.

- Ebene der Gesellschaft, einschließlich der Institutionen, Kunden, Gruppen, Normen und Standards.

Den einzelnen Ebenen lassen sich auch die der GIScience eng verwandten Wissenschaftszweige zuordnen: Die Individuumsebene (Kognitionswissenschaften, Umweltpsychologie, Linguistik) beschäftigt sich mit den Fragen, wie die Menschen die Welt konzeptualisieren und wie sie mit Hilfe von ihren Konzepten in der Welt handeln. Die Systemebene (Informatik) befasst sich vor allem mit dem Design von GIS, während in der Gesellschaftsebene (Ökonomie, Soziologie,

2 Projekt des National Centre for Geographic Information Analysis (1997-2000), dessen Ziel Formulierung der Forschungsthemen der GIScience war. 
Soziopsychologie, Geographie, Politikwissenschaften) die Anpassung von GIS an die Gesellschaft von Bedeutung ist.

Im Juni 1996 haben Vertreter von 29 Forschungsinstitutionen in den USA eine Liste mit den Forschungsthemen der GIScience erstellt (ANONYMUS 1996B).

(1) Erwerb räumlicher Daten und ihre Integration (spatial data aquisition and integration)

(2) Verteiltes Processing (distributed computing)

(3) Erweiterung der geographischen Repräsentation (extensions to geographic representations)

(4) Kognition (cognition of GI)

(5) Interoperabilität (interoperability of $G I$ )

(6) Skalen (Skala)

(7) Räumliche Analyse (spatial analysis in GIS environment)

(8) Zukunft der Geodaten-Infrastruktur (future of the spatial information infrastructure)

(9) Unsicherheit der Geodaten (uncertainty in geographic data and GIS-based activities)

(10) GIS und Gesellschaft (GIS and society)

Im Januar 1999 hat in den USA ein Workshop der National Science Foundation über Geographic Information Science und Geospatial Activities stattgefunden, dessen Ziel eine Beurteilung der Notwendigkeit der Grundlagenforschung in dieser neuen Fachdisziplin war. Während des Workshops wurden zwei wichtige Forschungsrichtungen definiert:

Grundlagenforschung in GIScience und

Forschung durch Anwendung von GIS.

Ein GIS stellt ein mächtiges Werkzeug für die Forschung in Umwelt- und Sozialwissenschaften dar: "GIS can support both exploratory and confirmatory analysis, provide tools for both inductive and deductive appoaches, and support both scientific research and the implementation of public policy based on GIS models" (MARK 1999A). Die Anwendung der Technologie wird jedoch durch verschiedene Faktoren behindert, die ihren Ursprung einerseits in der Natur der Geodaten und der Implementierung der Technologie, anderseits in der Ablehnung durch den Benutzer haben. Die mangelhafte Interoperabilität und Metadatendokumentation, die Unterschiede der Skala und Auflösung verschiedener Datensätze, fehlende Unterstützung der Dimensionalität und Temporalität der Daten sowie mangelhafte Benutzerfreundlichkeit der GIS sind die Hindernisse einer breiteren Anwendung der Technologie. Die Beseitigung dieser Hindernisse würde der GIS Technologie eine wichtige Rolle bei der Beschleunigung der Ideendiffusion zwischen den Disziplinen zuordnen.

Grundlagenforschung der GIScience beschäftigt sich mit komplexen Problemen, deren Lösung multidisziplinäre Ansätze verlangt. Die Hauptgefahr dieser Forschung ist jedoch das Risiko "of falling through the cracks between traditional disciplines, especially in the absence of targeted funding to support it" (MARK 1999A). Die Grundforschungsthemen der GIScience umfassen Themen wie Integration, Skala, Prozessmodelle und Nutzbarkeit. Die zusätzlichen Themen betreffen Repräsentation, Unsicherheit, Kognition, Simulation und Daten. 


\section{Geographische Informationstechnologie und -verarbeitung}

\subsection{Geographisches Informationssystem (GISystem)}

Geoinformationssysteme (geographische Informationssysteme, Geographic Information Systems) integrieren die räumlichen und a-räumlichen Informationen zu einer Einheit. In verschiedenen Fachdisziplinen hat sich eine ganze Reihe Spezialisierungen von solchen Systemen entwickelt: Rauminformationssysteme (RIS), Landinformationssysteme (LIS), Umweltinformationssysteme (UIS), Netzinformationssysteme (NIS) usw. Die charakterisierende Eigenschaft aller räumlichen Informationssysteme ist der Raumbezug. Ihre gemeinsame Funktionalität besteht in der Eingabe, Speicherung, Manipulation und Darstellung räumlicher Daten (BATTY \& DENSHAM 1996).

Ein Geoinformationssystem kann als eine Anwendung bestehend aus Hardware, Software, Daten und Benutzer, oder als eine Art Software verstanden werden (GoODCHILD 1997). Nach den genannten Aspekten werden drei GIS Definitionen unterschieden: Die Toolbox-basierte Definition nimmt die Verarbeitung räumlicher Daten als Differenzierungsmerkmal an. Die Grundfunktionalität von GIS umfasst im allgemeinen die Dateneingabe und -editierung, Datenkonvertierung, Datenspeicherung und -organisation, Datenmanipulation, Präsentation/Darstellung und räumliche Analyse. Die Datenbank-basierte Definition stellt den Unterschied der Speicherung und Verwaltung räumlicher Daten zu a-räumlichen Daten in den Vordergrund. Die Organisations-basierte Definition nimmt vor allem die Aufgabe der Organisationen und Menschen in der räumlichen Datenverarbeitung ins Visier.

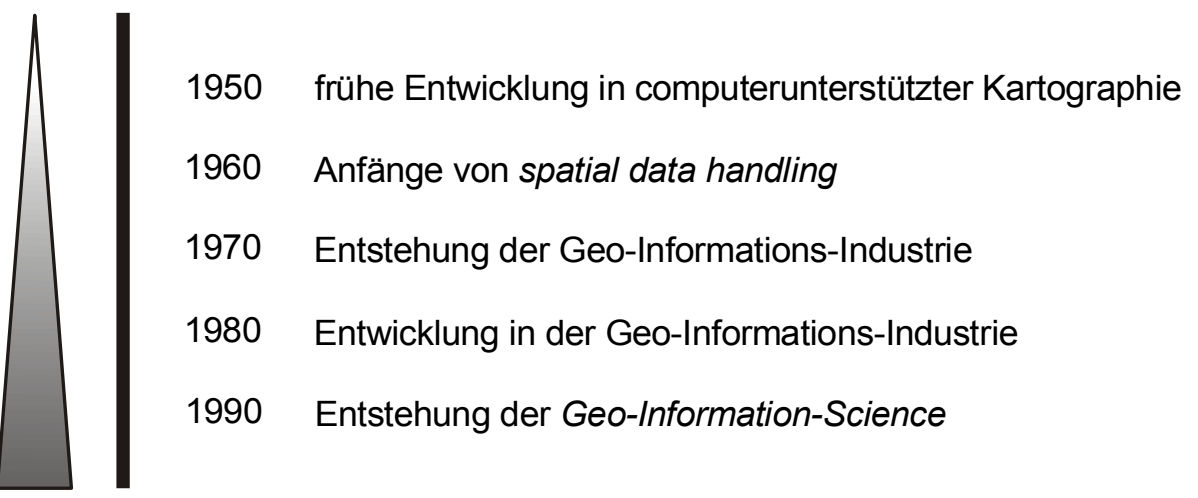

Abb. 9: Entwicklung Geographischer Informationssysteme.

CHRISMAN (1997) definiert ein GIS als eine organisierte Aktivität, bei welcher die Menschen

- Aspekte geographischer Phänomene und Prozesse messen,

- die Messungen in Form einer Datenbank repräsentieren, um räumliche Themen, Entitäten und Beziehungen hervorzuheben,

- diese Repräsentationen verarbeiten um weitere Größen abzuleiten und um neue Beziehungen durch Integration unterschiedlicher Quellen aufzudecken, und

- diese Repräsentationen transformieren, um sie an andere Systeme von Entitäten und Beziehungen anzupassen. 
Diese Aktivitäten spiegeln einen breiteren Kontext (Institutionen und Kulturen) wider, in welchem die Menschen arbeiten. GIS kann wiederum diese Strukturen beeinflussen.

Werden die räumlichen Konzepte in den Vordergrund gestellt, dann stellen GIS Systeme dar, welche die in Daten umgewandelten, einer Sichtsweise entsprechenden und situationsabhängigen räumlichen Konzepte nachbilden, speichern, analysieren und verwalten: "It is not only the technology that allows us to do things better and faster, it is new concepts and ideas, or a new paradigm, that leads us to do different things" (FEDRA 1994).

Die Entwicklung von GIS (Abbildung 9) in ihrer Anfangsphase in den 60er Jahren wurde mit dem Ziel vorangetrieben, durch die computergestützte Informationsverarbeitung die Kartographie zu erleichtern. Das erste GIS, „Canada Geographic Information System“, sollte die Automatisierung der Informationsverarbeitung gewährleisten, die in Form von Karten von Canada Land Inventory zusammengestellt wurden. Räumliche Analyse stand bei der Entstehung von GIS (GOODCHILD 2000) in Vordergrund und hat ihre Entwicklung in den 70er - 80er Jahren vorangetrieben. In dieser Zeit sah man in GIS eine integrierte Software Umgebung für räumliche Analyse. Nach dem die Computerpreise in den 80er Jahren gefallen sind, hat sich GIS in öffentlichen Institutionen und Universitäten sowie immer mehr privaten Firmen verbreitet: Der GIS Markt hat angefangen, zu wachsen. Heute sind weltweit $10^{5}$ Benutzer von GIS registriert, deren Preis größer als 10000 \$, und $10^{6}$ Benutzer von GIS, deren Preis kleiner als diese Grenze ist (GOODCHILD 2000).

Die neuere Entwicklung der Computer- und Softwaretechnologie in der Verbindung mit dem Fortschritt der Kommunikationstechnologien trugen dazu bei, dass sich die Nutzung der GIS immer breiter ausdehnte. Aus technischer Sicht zeichnen sich die Produkte der neuesten GIS-Generation durch die Unterstützung der Client/Server Architektur, den Einsatz von Standarddatenbanken mit in der Regel relationalen oder objektrelationalen Datenbankmanagementsystemen, die Entwicklung mit Hilfe objektorientierter Programmiersprachen, die Anbindung an Internet/Intranet, den Einsatz von case-tools zur Unterstützung der Benutzung (workflow Techniken), das Verfügen über eine Makro-Sprache zur Anpassung und Erweiterung der angebotenen Fähigkeiten aus. Für die Zukunft wird erwartet, dass die monolithischen Softwarelösungen durch eine Vielzahl kleiner interoperabler Module ersetzt werden. Der freie Datenfluss wird durch offene Spezifikationen ermöglicht.

\subsection{Geographische Modellierung}

Die Datenmodellierung geschieht im Zuge einer Abbildung (Morphismus) des Realitätsausschnitts in ein durch Hard- und Software realisiertes Informationssystem. Im Prozess dieser Abbildung werden Modelle entwickelt, die den Realitätsausschnitt mit zunehmender Präzision beschreiben (MATTHES \& SCHMIDT 1999). Ein Modell ist eine künstliche Konstruktion, in welcher ein Teil einer Quelldomäne (source domain) in einer Zieldomäne (target domain) repräsentiert wird (WORBOYS 1995). Die Bestandteile der Quelldomäne können Objekte, Beziehungen, Prozesse oder andere Phänomene sein. Diese werden im Modell vereinfacht und abstrahiert, und in der Zieldomäne abgebildet. 
Geographische Daten entstehen durch Abstraktion ${ }^{3}$ eines räumlichen Phänomens nach vorgegebenen Regeln, die den Inhalt (WAS) und die Weise der Repräsentation und Beschreibung (WIE) festlegen (AALDERS 1996). Diese subjektive Auswahl und Interpretation der realen Objekte bildet ein idealisiertes Weltaussehen, sog. 'nominal ground' (abstract view of the universe, terrain minimal). Die Subjektivität wird durch die Realitätsabstraktion und den Modellierungszweck verursacht.

Unter Geodatenmodellierung versteht man alle Schritte der Abstraktion, Abbildung und Speicherung der Geodaten. Sie umfasst sowohl geometrische als auch thematische Modellierung (vgl. BILL \& FRITSCH 1991). Die geometrische Modellierung wird definiert als "die Beschreibung, Bearbeitung und Speicherung der zugrundeliegenden Geometrie von raumbezogenen Objekten, indem analytische und approximierende Verfahren eingesetzt werden." (BILL \& FRITSCH 1991).

Das Konzept der Datenmodellierung stammt aus der Datenbanktheorie. Mit der Datenmodellierung wird die Struktur und Logik der Datenorganisation beschrieben. Die Datenmodelle sind Struktursprachen, die sich zur Beschreibung von Datenbeständen eignen (HÄTTENSCHWILER 1997). Ein Datenmodell besteht aus Beschreibung und Spezifikation von:

- Datentypen,

- Beziehungen und Abhängigkeiten,

- Beschränkungen,

- Grundoperationen und benutzerdefinierten Operationen.

Während der Datenmodellierung werden mehrere Modelle unterschiedlichen Abstraktionsniveaus benötigt: Im ersten Schritt werden alle relevanten Konzepte aus der Realitätsbetrachtung abstrahiert und in einem konzeptionellen Modell abgebildet. Die Realität wird simplifiziert, idealisiert, formalisiert und dadurch systematisiert. Die Objekte (entities), ihre Eigenschaften (attributes) und Beziehungen untereinander (relations) werden in dem konzeptionellen Modell schematisch dargestellt. Um die Semantik in leicht verständlicher Weise darzustellen, bedient man sich grafischer Hilfsmittel. Die am häufigsten angewandten Modelle sind ERM (EntityRelationship-Modell) und OOM (objekt-orientierte Modellierung). Das konzeptionelle Modell beschreibt die reale Welt unabhängig von der Implementierung und bildet die Grundlage für die nächste Abstraktionsebene.

Die Übertragung eines konzeptionellen Modells in eine Repräsentationsform (Datenmodell des Datenbanksystems) im Rechner geschieht mit Hilfe eines logischen Datenmodells. Ein Datenbankmodell beschreibt die Semantik von Deklarationen und Ausdrücken der Datenbanksprache. Es definiert die Strukturierungsmechanismen, Identifikationskonzepte und allgemeinen Operationen, die zur Konstruktion eines Datenbankschemas und zur Beschreibung von Datenbankoperationen zur Verfügung stehen (MATTHES \& SCHMIDT 1998). Zu den logischen Modellen zählen das objektorientierte, relationale oder objektrelationale Datenmodell, das Netzwerkmodell und das hierarchische Modell. Sie beschreiben die abstrakte Repräsentation der Daten in einem Datenbankmanagementsystem (DBMS). Die Struktur einer konkreten Anwendung wird als logisches Datenbankschema (BIETHAN et al. 1992) bezeichnet. Ein

\footnotetext{
${ }^{3}$ Abstraktion ist Hervorheben von essentiellen Charakteristiken eines Objektes, die es von allen anderen Objekten unterscheiden.
} 
Datenbankschema ist als Beschreibung eines Datenbestandes gleichbedeutend mit dem Begriff Metadaten.

Physische Datenmodelle verwenden Konzepte auf einem sehr niedrigen Abstraktionsniveau. Sie nehmen Bezug auf Details der Datenspeicherung und der Implementierung des Datenbanksystems. Das physikalische Schema bleibt dem Benutzer verborgen, hier werden spezifische Datenstrukturen des DBMS benutzt.

Nach dem Grad der Abstraktion werden im Rahmen der räumlichen Datenmodellierung mehrere Schritte unterschieden (EGENHOFER 1996). Die räumlichen Konzepte beziehen sich auf das menschliche Denken und Verstehen. Sie sind die mentalen Modelle des Raumes. Für unterschiedliche Typen des räumlichen Phänomens wurden unterschiedliche Konzepte entwickelt: objektorientierte (entity-oriented concepts) und raumorientierte Konzepte (spaceoriented oder position based concepts). Beispiele für objektorientierte räumliche Konzepte sind Grenzenrepräsentation, euklidische Geometrie, Graphen, objekt-orientierte Partitionen usw. Raumorientierte räumliche Konzepte sind beispielsweise Punktmenge, zellbasierte Partition und Informationsschichten (SCHNEIDER M. 1997).

Die räumlichen Konzepte sind nicht direkt implementierbar. Die räumlichen Datenmodelle (spatial data models) RDM befassen sich mit den räumlichen Daten im Hinblick auf ihre Speicherung und Formalisierung. Auf einer abstrakten Ebene werden die Objekte, die zur Handhabung der geometrischen Daten nötig sind, ihre Methoden und ihr Verhalten definiert. Die Formalisierung geschieht mit Hilfe mathematischer Methoden für die Beschreibung der Struktur räumlicher Daten und die Semantik ihrer Operationen. Beispiele für Datenmodelle sind (erweiterte) relationale oder objektorientierte Modelle.

Die Implementation eines RDM erfolgt mit Hilfe von räumlichen Datenstrukturen (RDS). Die räumlichen Datenstrukturen (spatial data structures) klären die Implementierungsfragen voriger Beschreibungen. Eine RDS beschreibt nur eine mögliche Implementierung (array 1d, 2d, strip tree, quad tree). Die Datenstrukturen werden Vektor- und Rastermodelle genannt, je nachdem, ob sie die objekt- oder feldbasierten Konzepte unterstützen.

Aus der Sicht des modellierten Zusammenhanges der räumlichen Information werden drei Ebenen der Komplexität beschrieben (SCHNEIDER, M. 1997), auch als context level bezeichnet. Jede Ebene baut auf der vorherigen auf. Die räumliche (geometrische) Modellierung (spatial modelling) stellt einen Designprozess der abstrakten Objektrepräsentation dar. In ihm werden die geometrische Struktur, die Ausdehnung und Form der Objekte und ihre räumlichen Beziehungen beschrieben. Die Objektmodellierung (object modelling) beschreibt die Modellierung und Organisation realer Objekte, auch räumlich-referenzierte Objekte genannt. Diese umfassen räumliche und a-räumliche Komponenten. Die Strukturmodellierung (structure modelling) erweitert die vorige Ebene um die Anordnungsstruktur (connectivity structure). Diese beschreibt eine Menge räumlich referenzierter Objekte mit ihren Eigenschaften und den Bedingungen ihrer Anordnung (Konfiguration).

Die thematische Modellierung unterscheidet Layer und objekt-bezogene Konzepte. Im Layer-Konzept repräsentiert jede Informationsschicht (Layer, Schichten, Folien, coverages) einen thematischen Ausschnitt vom gleichen Untersuchungsgebiet (SCHNEIDER R. 1993). Ein Layer entspricht einer thematischen Karte. Jedes einzelne Attribut wird in einer separaten 
Informationsschicht erfasst. Bei einer Anwendung werden dann ausgewählte Schichten digital deckungsgleich übereinandergelegt. Alle Schichten müssen das gleiche Koordinatensystem, den gleichen Maßstab und den gleichen Raumausschnitt besitzen (bzw. entsprechend transformiert werden). Häufig organisiert man die Schichten so, dass in jeder Schicht nur Geoobjekte gleicher geometrischer Dimension und gleicher Klassenzugehörigkeit enthalten sind. Die Modellierung entspricht dem "top-down" Konzept vom Allgemeinen zum Speziellen. (STREIT 2000)

Der objekt-bezogene Ansatz ist ein neuartiges Konzept, das sich auf das objekt-orientierte Paradigma stützt. Die Objekte modellieren einen bestimmten Ausschnitt der Erdoberfläche in bezug auf eine oder mehrere Eigenschaften (Themen). Jedes Geoobjekt besitzt seine eigene Geometrie, Topologie, Thematik und Dynamik sowie seine eigenen Methoden. Die geometrische Komponente beschreibt Ort und Ausdehnung des durch das Objekt repräsentierten Realitätsbereiches. Die thematische Komponente enthält eine qualitative oder quantitative thematische Charakterisierung (SCHNEIDER R. 1993). Die Objekte sind gegenüber anderen Objekten gekapselt und korrespondieren über Nachrichten miteinander. Objektklassen stellen generalisierte Konzepte für Objekte dar; ein konkretes Objekt besitzt als 'Instanz' seiner Klasse alle Attribute und Methoden dieser Klasse, jedoch z.B. spezifische Attributwerte. Die Modellierung entspricht dem "bottom-up" Konzept (STREIT 2000).

\subsection{Geo-Datenbanksysteme}

Geodatenbanksysteme sind Nichtstandard-Datenbanksysteme, die Objekte mit Raumbezug (Geoobjekte) verwalten. Die spezifischen Eigenschaften dieser Objekte sind die absolute oder relative Position im Raum, die Ausdehnung und die Form. Die Geo-Datenbanksysteme unterscheiden sich von den Standard-DBMS durch räumliche Datenmodelle und Anfragesprachen sowie durch eine adäquate physische Organisation der Objekte (BRINKHOFF 1994). Eine räumliche Datenbank ist eine Menge räumlich referenzierter Daten, die ein Modell der Realität darstellt (NYERGES 1997).

Ein Geodatenbanksystem wird von GÜTING (1999) durch folgende Eigenschaften charakterisiert:

- Ein räumliches Datenbanksystem ist ein Datenbanksystem.

- Es bietet räumliche Datentypen (spatial data types, SDT) in ihrem Datenmodell und eine räumliche Abfragesprache an.

- Es unterstützt die räumlichen Datentypen in ihrer Implementation mindestens mit räumlicher Indexierung und effizientem Algorithmus für den räumlichen Verbund (Spatial Join).

Der Begriff 'räumliche Datenbanksysteme' (spatial, geometric, geographic DBMS) wird für die objektorientierte Betrachtung des Raumes (Raum als Menge der Objekte und ihrer Beziehungen) verwendet. Für feldbezogene Repräsentationen des Raumes werden die Begriffe pictorial oder image database system benutzt (GÜTING 1999).

Geodatenbanken verwenden räumliche Datentypen, um raumbezogene Objekte speichern und verwalten zu können. Die Auswahl und Bearbeitung der Daten steht im Vordergrund der räumlichen Datenbanktechnologie. Im Umgang mit Geodaten ist die graphische Darstellung und 
Kombination der Objektmengen als ganze Speicherstrukturen oder als Anfrageergebnisse, sowie eine graphische Interaktivität mit diesen unerlässlich. Dazu benötigt man Werkzeuge zur Anfrage des Bildschirminhaltes. Die graphischen Darstellungen müssen die Bedingungen einer Kontextdarstellung in bezug auf Einbeziehung der Hintergrundinformationen, Darstellung der Legende, Beschriftungen, Auswahl des Maßstabes und Definition der Ausdehnung erfüllen. Für die Anfragen räumlicher Daten ist die Implementation einer räumlichen Algebra in der Anfragesprache eines DBMS nötig.

Ein räumliches Attribut bezeichnet eine Eigenschaft der geometrischen (räumlichen) Komponente eines Geoobjektes. Die möglichen Ausprägungen eines räumlichen Attributes bilden die Domäne eines räumlichen Prädikates (BREUNING 1995). Räumliche Beziehungen sind Relationen, die ein räumliches Prädikat auswerten. Eine räumliche Selektion bezeichnet die Auswahl von Geoobjekten aus einer Geoobjektmenge, die durch ein oder mehrere räumliche Prädikate spezifiziert ist. Die räumliche Projektion ist die Ausblendung (mindestens) eines Attributes der Geoobjektmenge. Der räumliche Verbund bezeichnet die Verknüpfung von Geoobjekten aus zwei Geoobjektmengen anhand des Vergleiches eines ihrer räumlichen Attribute. Die lokale Anfrage ist eine Anfrage an ein einzelnes Geo-Objekt, während eine globale Anfrage mehrere Geoobjekte und ihre Anordnung einbezieht. Eine räumliche Operation ordnet einer Auswertung des räumlichen Prädikates eine Menge der reellen Zahlen oder neu berechneten Geoobjekte zu.

Die Geoobjekte werden auf einem Sekundärspeicher gespeichert, der in Seiten gleicher Größe eingeteilt ist. Um ein Geoobjekt einzulesen oder abzuspeichern, muss eine ganze Seite eingelesen werden (seitenweiser Zugriff). Auf einer Seite kann je nach der Komplexität der Geoobjekte eine unterschiedliche Anzahl von Geoobjekten gespeichert werden (von mehreren bis zu einem Teil eines Geoobjektes). Die Zuordnung von einem Geoobjekt zu einer Seite erfolgt über eine Zugriffsstruktur. Die Zugriffstrukturen organisieren die Objekte der Datenbank anhand der Ausprägung ihres Schlüsselattributes (Index). Die Indexstrukturen organisieren eine dynamische Menge von Objekten auf einem seitenorientierten Sekundärspeicher und unterstützen somit die effiziente Bearbeitung der Anfragen und die Operationen auf dem Datenbestand. Ihre Aufgabe ist es, möglichst viele Objekte von vornherein von der Ergebnismenge auszuschließen und somit die Anzahl der Seitenzugriffe zu beschränken.

\subsection{Geographische Informationsverarbeitung}

Eine komplexe Verarbeitung von Geodaten ist die Aufgabe der geographischen Informationsverarbeitung oder Geoinformationsverarbeitung (spatial data handling).

Der Definition von GIS nach stellt die Ableitung neuer Informationen aus den bestehenden räumlichen Datenbeständen eine der Hauptaufgaben eines GIS dar. Die Geodatenverarbeitung umfasst die Operationen zur Umstrukturierung, Generierung, und Auswertung der Information. Diese Operationen reichen von einfachen bis zu komplexen analytischen Vorgängen. Alle verwenden den Raum als gemeinsame Referenzbasis. Die räumliche Perspektive erlaubt, auf die relative Position der Objekte, Ereignisse und Prozesse zuzugreifen und diese miteinander zu verbinden. (vgl. FISCHER et al. 1996). Die Operationen der Geodatenverarbeitung müssen den besonderen Charakter der Geodaten berücksichtigen. 
Die Analyse und Modellierung räumlicher Strukturen und Prozesse (spatial analysis, spatial modelling) ist eine grundlegende Aufgabe aller Geowissenschaften. Trotz (und vielleicht auch wegen) der Vielfalt der GIS Anwendung und daher eines breiten Spektrums der Interessen an der Implementation von analytischen Werkzeugen in GIS haben die gängigen GIS Produkte dieser Aufgabe nur in kleinem Maße Rechnung getragen. Die Interessen waren eine Treibkraft der Implementation, während die Vielfalt oft zur Hemmung der Entwicklung beiträgt.

Über keine der Themenbereiche von GIScience wurde in den letzten Jahren mehr diskutiert als über die räumliche Analyse. Die Notwendigkeit zur Erweiterung der Kernfunktionalität der Geodatenverarbeitung war neben der offenen Möglichkeit ihrer individuellen Anpassung und Ergänzung in dieser Diskussion unumstritten. "GIS clearly needs stronger analysis and modeling capabilities if it is to meet its potential as a general-purpose tool for handling spatial data" (GOODCHILD 1992 S.409). Die Diskussion betraf die Festlegung der benötigten allgemeinen Operationen, den Durchschnitt der Bedürfnisse von Einzeldisziplinen und ihre Implementationsart. Die minimale effektive Menge der Operationen sollte in Form allgemeiner analytischer und modellierungstechnischer Werkzeuge in GIS implementiert und um eine Schnittstelle zu den fachspezifischen Modulen erweitert werden. Diese Entwicklung scheint für eine Weiterentwicklung von GIS nicht nur erwünscht, sondern absolut notwendig zu sein. "New era of computation with computer speeds and memory capabilities moving into ,terracomputing" domains will help create an environment where spatial analysis will not merely be of increasing interest and value but in some areas it will be an absolute necessity" (OPENSHAW \& CLARKE 1996).

Dem Optimismus im Bereich räumlicher Analyse auf der einen Seite ("The emphasis on GIS database creation and systems building will be replaced by a new concern with analysis" (OPENSHAW \& ClaRKE 1996) steht Skepsis auf der anderen gegenüber. Die Implementation aufwendiger analytischer und modellierungstechnischer Funktionalität muss an die Bedürfnisse der Benutzer angepasst werden. Der Umgang mit den analytischen Funktionen in einer GIS Umgebung ist nicht immer sachgerecht, manchmal sogar fehlerhaft (UNWIN 1997). UNWIN (1997) formuliert drei Annahmen der räumlichen Analyse, wie sie sich aus der Fachliteratur ergibt:

- Es gibt etwas, was als räumliche Analyse definiert werden kann.

- Sie ist nützlich in einer GIS Umgebung.

- Sie wird (in der Zukunft) angewendet werden: 'Things will happen' .

Die allgemeinen Prinzipien eines künftigen Technologiedesigns der GISable räumlichen Analyse werden von OPENSHAW \& CLARKE (1996) weiter ausgeführt und in UNWIN (1997) diskutiert:

- GISfähige räumlich-analytische Methoden müssen große Datenumfänge verarbeiten können.

- GISfähige Werkzeuge der Analyse und Modellierung müssen vom Referenzgebiet unabhängig sein.

- GIS relevante Methoden müssen sensitiv für die spezielle Natur der Geoinformation sein.

- Das Ergebnis der Analyse muss in einer Karte visualisierbar sein.

- GISfähige räumliche Analyse ist allgemein.

- GISfähige räumliche Methoden müssen nützlich und berechenbar sein.

- Die Einfachheit der Benutzung und Verständlichkeit ist sehr wichtig.

- GISfähige Analyse muss eine sichere Technologie sein.

- GISfähige Methoden sollen für die Anwendungen nützlich sein. 
Die Begriffe Analyse und Modellierung werden oft synonym oder ohne klare Definition und Trennung benutzt.

Von TOMLIN (1990) und von FALCIDIENO et al. (1996) wird die Dichotomie deskriptive/präskriptive Modellierung benutzt. Die deskriptive Modellierung (desciptive modeling) ist ein bottom-up Verfahren der Wissensaktualisierung durch Extraktion neuer, im vorhandenen Datensatz implizit enthaltener Information. Diese Modellierungsart wird durch die Gewinnung einer high-level Information aus dem low-level geometrischen Modell charakterisiert. Die Aufgabe der präskriptiven (deklarativen) Modellierung ist die Repräsentation des Wissens, die Simulation der Prozesse, das Treffen von Urteilen und die effektive Beschreibung eines geographischen Phänomens durch seine Eigenschaften und constraints (FALCIDIENO et al. 1996).

Der Begriff "unified geographic modeling" wird von HAO (1996) benutzt, um eine gemeinsame Basis für räumliche und a-räumliche, statische und dynamische Daten- und analytische Modellierung zu bezeichnen. Die räumliche Modellierung betont die Wichtigkeit der Position (WO sind die Dinge). Die temporale (dynamische) Modellierung ist auf die Ereignisse (WANN werden die Dinge geändert) ausgerichtet. Die Datenmodellierung beschreibt WAS es gibt und WIE die Dinge miteinander verbunden sind. Die analytische Modellierung versucht die Beziehungen der Dinge aufgrund eines Prinzips mathematisch zu erklären.

Eine sehr verbreitete Ansicht fasst die analytischen und modellierungstechnischen Operationen der Geoinformationsverarbeitung unter dem Begriff spatial analysis zusammen (FISCHER et al. 1996). Diese kann ihrer Zusammensetzung nach in statistische räumliche Datenanalyse (statistical spatial data analysis) und räumliche Modellierung (spatial modeling) gegliedert werden. In SCHUT (1998) findet man eine andere Unterscheidung nach Grundoperationen: Beschreibung (description), Position (location), Aggregation (summary), Analyse (analysis), Testen (model validation), und voraussagende Modellierung (predictive modelling).

Im weiteren werden die Operationen der Geoinformationsverarbeitung in drei Bereichen behandelt:

- räumliche Operationen zur Umstrukturierung der Daten und Informationen (Datenmanipulation).

- räumliche Analyse zur Ableitung von Informationen.

- räumliche Modellierung und Simulation zur Überführung der Informationen in Wissen

\subsubsection{Datenmanipulation}

Die Funktionalität der Manipulation räumlicher Daten kann als eine Menge elementarer Operationen verstanden werden, mit Hilfe derer Geodaten gewonnen, selektiert oder umstrukturiert (geändert oder neu berechnet) werden. Sie schließen die thematischen und geometrischen Attribute ein. Die Aufgabe der Manipulation räumlicher Daten ist ihre Vorbereitung für die kartographische Präsentation und die räumliche Analyse (YUE-HoNG 1997).

Zur Gliederung räumlicher Operationen wurden mehrere Versuche unternommen. Dieses hat wegen der Vielfalt der Operationen zu keiner einheitlichen Typisierung geführt. Eine gängige Gliederung (YuE-Hong 1997), die schon aus Tomlins' MapAlgebra (TOMLIN 1990) bekannt ist, 
beruht auf der Anzahl der verwendeten Datenstrukturen: horizontale (single layer) und vertikale Operationen (multiple layer). Die erste Klasse umfasst alle Operationen, die an einer geographischen Informationsschicht durchgeführt werden können, um die vorhandenen Objekte in ihrer Anordnung zu ändern. Der zweiten Klasse der Operationen liegt der räumliche Verbund zugrunde, der die räumlichen Objekte mehrerer Informationsschichten verbindet und die Verbindung quantifiziert.

BURROUGH (1992) unterscheidet 8 Operations-Klassen, wobei als das Gliederungskriterium die Berücksichtung der räumlichen Umgebung, der zeitlichen Veränderung und der Unsicherheit der Eigenschaften der Geoobjekte verwendet werden. FISCHER \& NIJKAMP (1991) ziehen andere Kriterien zur Gliederung der fundamentalen und allgemeinen Typen der 'räumlichen Analyse' vor. Diese umfassen die Art der Eigenschaft (thematisch, räumlich) und die Anzahl der beteiligten Objekte und Klassen. Eine andere klassische Gliederung der Operationen und der analytischen Vorgänge betrachtet die Problemfragestellung und die zugrundliegenden Datenmodelle (BEHR 1997a).

Die universellen High-Level GIS Operatoren (ALBRECHT 1994) wurden als Herzstück eines virtuellen GIS (VGIS) angewendet, das einen Aufbau auf einem GIS darstellt und unabhängig von ihm die Verarbeitung der Geodaten auf der semantischen Ebene mit Hilfe einer graphischen Oberfläche und einer Art Casetool erlaubt (siehe Tabelle 2).

Tab. 2: Universelle GIS Operationen nach ALBRECHT (1995).

\begin{tabular}{|c|c|c|c|c|}
\hline Search & Interpolation & $\begin{array}{l}\text { Thematic } \\
\text { Search }\end{array}$ & Spatial Search & (Re-)classifikation \\
\hline $\begin{array}{l}\text { Location } \\
\text { Analysis }\end{array}$ & Buffer & Corridor & Overlay & Thiessen/Voronoi \\
\hline $\begin{array}{c}\text { Terrain } \\
\text { Analysis }\end{array}$ & Slope/Aspect & $\begin{array}{c}\text { Catchment/ } \\
\text { Basins }\end{array}$ & Drainage/Network & $\begin{array}{l}\text { Viewshed } \\
\text { Analysis }\end{array}$ \\
\hline $\begin{array}{l}\text { Distribution/ } \\
\text { Neighborhood }\end{array}$ & $\begin{array}{c}\text { Cost/Diffusion/ } \\
\text { Spread } \\
\end{array}$ & Proximity & Nearest Neighbor & \\
\hline $\begin{array}{c}\text { Spatial } \\
\text { Analysis }\end{array}$ & $\begin{array}{c}\text { Multivariate } \\
\text { Analysis }\end{array}$ & $\begin{array}{c}\text { Pattern/ } \\
\text { Dispersion }\end{array}$ & $\begin{array}{c}\text { Centrality/ } \\
\text { Connectedness }\end{array}$ & Shape \\
\hline Measurements & Measurements & & & \\
\hline
\end{tabular}

\subsubsection{Räumliche Analyse}

Die räumliche Datenanalyse ist eine Menge von Techniken, gedacht zur Unterstützung der räumlichen Perspektive der Daten (GOODCHILD 1992). Sie vereint die Analyse und Synthese von raumbezogenen Daten (BILL \& FRITSCH 1996). Eine eindeutige Klassifikation gibt es ähnlich wie bei der Manipulation nicht. "If one considers all available techniques and models that have been used in the spatial science in the past or that could potentially be used, one realizes it is virtually impossible to find a classification of low dimension that would encompass all of them" (ARENTZE et al. 1996, S.188).

Das spezifische Problem einer räumlichen Analyse ist das Modifiable Areal Unit Problem. Der Begriff "MAUP" bezeichnet die Beeinflussung der korrelativen und autokorrelativen Beziehung der Variablen, welche auf die Größe (Aggregation) und Form (Zonierung) der Bezugseinheiten und auf die Abgrenzung des Untersuchungsgebiets (Teilmenge) zurückzuführen ist. Die Bildung 
der Bezugsregionen unterschiedlicher Aggregationsstufe oder Zonierung enthält ein Maßstabsproblem und das Problem der Grenzziehungen.

Allgemein unterscheidet GoODCHILD (1992) die Techniken der räumlichen Analyse nach der verwendeten Information in eine Analyse räumlicher Verteilung (nur die positionsbezogenen Informationen) und eine Analyse räumlicher Variation der Attributswerte (eine Kombination der positionsbezogenen und attributsbezogenen Daten). Weitere Kriterien sind die Art der Geodaten (Methoden für die räumlich-kontinuierlichen oder mindestens quasi-kontinuierlichen Daten versus Methoden für die Objekt-basierten Daten) und die Aufgabe der Analyse (deskriptive Methoden vs. schließende Methoden). Nach ARENTZE et al. (1996) basiert die Gliederung auf der Aufgabe der Analyse:

- Die Deskriptive Analyse beschreibt die Verteilung der Daten.

- Die Assoziative Analyse sucht nach einer funktionalen Beziehung zwischen Variablen.

- Die Datenreduktionsmethoden reduzieren die Anzahl der Variablen auf eine kleine Anzahl zugrundliegender (latenter) Dimensionen oder gruppieren die beobachteten Fälle in eine kleine Anzahl von Klassen.

Nach FISCHER et al. (1996) umfasst die statistische räumliche Datenanalyse folgende Bereiche:

- Stichprobennahme (sampling)

- Analyse der aufgrund der unterschiedlichen Skalierungs- und Abgrenzungseinheiten unkompatiblen Information

- Exploratory spatial data analysis (ESDA)

- Confirmatory oder Explanatory spatial data analysis CSDA

Die Explorative Datenanalyse (EDA) folgt dem Ziel, die Eigenschaften von Daten zu identifizieren, um ein Muster und Assoziationen zu erkennen, um Hypothesen zu formulieren und um verschiedene Aspekte der Modelle abzuschätzen. Sie basiert auf graphischen und visuellen Methoden und benutzt numerische Techniken, die statistisch robust sind, d.h. die nicht von extremen Werten beeinflusst werden. Sie beruht auf deskriptiven Methoden, nicht auf dem Testen formaler Hypothesen. Das Ziel der EDA ist eine Annäherung an originale Daten unter Benutzung von einfachen, intuitiven Methoden.

Die Explorative Räumliche Datenanalyse (ESDA) erweitert die EDA um die Untersuchung der räumlichen Eigenschaften von Daten. Sie benötigt eigene Methoden für die Untersuchung der räumlichen Muster, die Formulierung von Hypothesen, welche auf dem räumlichen Bezug der Daten basieren, und für die Schätzung der räumlichen Modelle. Die zentrale Idee der ESDA ist es, die numerischen und graphischen Prozeduren mit der Karte zu verknüpfen. Mit modernen graphischen Oberflächen ist das meistens mit "brushing" möglich.

Die Bestimmung der räumlichen Assoziation oder räumlichen Autokorrelation ist das Herzstück der ESDA (ANSELIN \& BAO 1997). Bei den Ansätzen hierzu ist zwischen zwei Richtungen zu unterscheiden: die geostatistischen distanzbasierten und die nachbarschaftsbasierten Methoden. Während die erste Gruppe auf einem vordefinierten Distanzsystem basiert, verlangt die zweite Gruppe eine Definition der Nachbarschaftsstruktur (Topologie oder räumliche Anordnung) in Form einer Gewichtungsmatrix.

Die Maße der globalen räumlichen Autokorrelation (Morans I, Gearys c oder Gamma Indizes) aggregieren die Beschreibung der gefundenen Abhängigkeit der Daten in einem einzigen Wert. 
Die Indizes der lokalen räumlichen Autokorrelation (LISA local indicators of spatial assotiation ANSELIN 1995) zeichnen sich durch die Fähigkeit, die lokale räumliche Assoziation und ihr Muster zu erkennen und die globalen Indizes auf ihre Stabilität zu überprüfen, aus $\left(\mathrm{G}, \mathrm{G}^{*}\right.$, local Moran scatterplot).

Viele Ansätze beabsichtigen keine Gliederung, sondern beschränken sich auf eine (meist unkomplette) Aufzählung der analytischen Methoden. FISCHER \& NiJKAMP (1991) zählten auf: Exploratory point pattern und area data analysis, Regionalisierung, räumliche Regressionsmodelle, raum-zeitliche statistische Methoden für Erwerb (acquisition), Speicherung (storage), Analyse (analysis) und Prädiktion (forecasting), location-allocation Modelle, optimale Trassenfindung (route procedures), räumliche Suche, räumliche Dateninterpolation, Thiessen Modelle, Gaussian Diffusionsmodelle, Techniken für Monitoring der Fehlerpropagation (Monte Carlo simulation), fuzzy set basierte analytische Prozeduren, auf künstlicher Intelligenz basierte räumliche Analyse. Andere Klassifikation haben OPENSHAW \& CLARKE (1996) entwickelt.

Die Visualisierung als die Transformation von Daten in ein sichtbares Bild zur Unterstützung der Exploration (Erkundung), Kognition (Erkennen) und Explanation (Erklärung) von Strukturen und Prozessen zählt auch zu den analytischen Methoden der Geoinformationsverarbeitung.

\subsubsection{Modellierung und Simulation}

Prozessmodellierung und Simulation sind theoretische Konzepte und Methoden zur Beschreibung, Abbildung und Simulation der Funktionsweise der realen Prozesse. Räumliche Modellbildung kann als vertretbare und im Sinne der problembezogenen Handhabbarkeit von Problemstellungen geradezu erforderliche Reduzierung räumlicher Komplexität betrachtet werden. Raumbezogene Simulation ist eine Vorwegnahme oder auch Nachstellung von unterschiedlich konkreten räumlichen Situationen und raumbezogenen Prozessen (VOIGT 1999). Sie integriert die unterlegenen Ebenen der Geodatenverarbeitung in eine komplexe Abbildung der modellierten Realität.

Die Modellierung und Simulation stellt eine komplexe Komposition der Operationen und Analysen und ihrer Ergebnisse dar, mit welcher Information in Wissen überführt wird. Sie umfasst Analyse und Verstehen des beobachteten Phänomens, Testen von Hypothesen und Theorien, Prognose des räumlich-zeitlichen Systemverhaltens unter verschiedenen Szenarien und Entdecken von neuer Information über ein räumliches Phänomen durch ein computergestütztes Experiment (MiTASOVA \& MiTAS 1998).

Die realen räumlichen Prozesse sind meistens sehr komplex und enthalten oft nicht-lineares Verhalten, stochastische Komponenten und Rückkopplung über räumliche und zeitliche Skalen. Die Modelle können deshalb die Prozesse nur auf einer bestimmten Stufe der Vereinfachung repräsentieren (MITASOVA \& MITAS 1998). Nach der Art der Modelle können deterministische und stochastische Modelle, regelbasierte Modelle, und multi-agent Simulationen der Systeme unterschieden werden. Nach dem räumlichen Datentyp und dem Typ der räumlichen Verteilung können Prozessmodelle das Verhalten von Phänomenen durch homogene räumliche Einheiten, Netzwerke, Punkte und ihre Kombinationen repräsentieren. Die räumliche Interaktion kann ihrer Natur nach vom Typ Fluss, Wucherung, Populationsdynamik und intelligente Agenten sein. Schließlich kann die räumliche Ausdehnung und Skala des modellierten Phänomens lokale, regionale, globale oder geschachteltete Modelle unterscheiden. BURROUGH (1997) gliedert die 
Modellanwendung in GIS in die regelbasierten Modelle, empirischen Regressionsmodelle, deterministischen und stochastischen physischen Modelle.

Nach FISCHER \& NiJKAMP (1992) umfassen die im Rahmen eines GIS angewendete räumlichen Modellarten

- kartographische Modellierung,

- agentenbasierte Modelle zur Simulation individuellen räumlichen Verhaltens,

- zelluläre Automaten zur Beschreibung und Simulation räumlicher Prozesse,

- fuzzy set Methodik für qualitative räumliche Modellierung und Simulation,

- räumliche Entscheidungsunterstützungsmodelle und -systeme.

Die Simulationsmodelle haben die Aufgabe, Variablenwerte durch iterative Veränderung einem Simulationsziel anzunähern (MANDL 1998). Neben den Zustandsvariablen benötigt man eine Variable (meist die Zeit), die das Simulationssystem dynamisch und inhaltlich bestimmt. Als Beispiel der räumlich-diskreten Simulationsmodelle können zelluläre Automaten ZA (cellular automata CA) betrachtet werden. Ein zellulärer Automat ist ein System von räumlichen Einheiten, meist Rasterzellen, mit einer Menge von möglichen Zuständen. Die Bestandteile von ZA sind Zustand (state), Nachbarschaft (neighbourhood) und Funktion (GREEN 1993). In diskretem Zeitschritt ändert die Übergangsfunktion den Zustand dieser Einheiten. Die Übergangsfunktion berücksichtigt die Zustände der Einheiten in einer festgelegten Nachbarschaft. Ein ZA System hat einen globalen Anfangszustand und sich im Laufe der Simulation entwickelnde, stabile, periodische oder völlig unregelmäßige globale Zustände, die das Verhalten des Systems charakterisieren (MANDL 1998).

Das Konzept der intelligenten Agenten stammt aus dem Bereich der künstlichen Intelligenz. Als ein Agent wird ein System bezeichnet, das die bestmögliche Erfüllung vorgegebener Ziele in einer komplexen dynamischen Umgebung zu erreichen versucht. Ein Agent verfügt über Rezeptoren, mit welchen er den Umweltzustand wahrnehmen kann, und kann eigenständig handeln. Ein räumlicher Agent kann in einer räumlichen Repräsentation handeln. Seine Anwendungsmöglichkeiten umfassen die Simulation und Entscheidungsfindung in einer räumlichen Domäne. 


\section{Räumliche Situation}

Eine räumliche Situation beschreibt eine Konfiguration von räumlichen Objekten in ihren individuellen Eigenschaften sowie ihren gegenseitigen Beziehungen. Im folgenden Kapitel wird auf die Eigenschaften räumlicher Objekte und die Theorie der räumlichen Beziehungen ausführlicher eingegangen.

\subsection{Geoobjekt}

\subsubsection{Eigenschaften der Geoobjekte}

Ein Geoobjekt (feature) ist ein auf einen räumlichen Ausschnitt der Erde bezogenes reales oder gedankliches Objekt (STREIT 2000). Die Verwendung dieses Begriffes in der fachlichen Literatur ist nicht eindeutig. Im weiteren werden unter diesem Begriff sowohl das reale Urbild als auch das digitale (mit Geodaten beschriebene) Bild gefasst. Die Modellierung der Geoobjekte erfolgt durch Abstraktion, die alle relevanten Konzepte (Geometrie, Thematik, Verhalten) enthält, während unrelevante außer Acht bleiben.

Der Raumbezug ist die wichtigste charakteristische Eigenschaft der Geoobjekte. Durch die Einführung eines einheitlichen räumlichen Bezugssystems ist es möglich, jede konkrete, physische, geometrisch oder begrifflich begrenzte Einheit der Erdoberfläche als raumbezogenes Objekt in einem Modell abzubilden und jeder dieser Einheiten eine geometrische Dimension zuzuweisen.

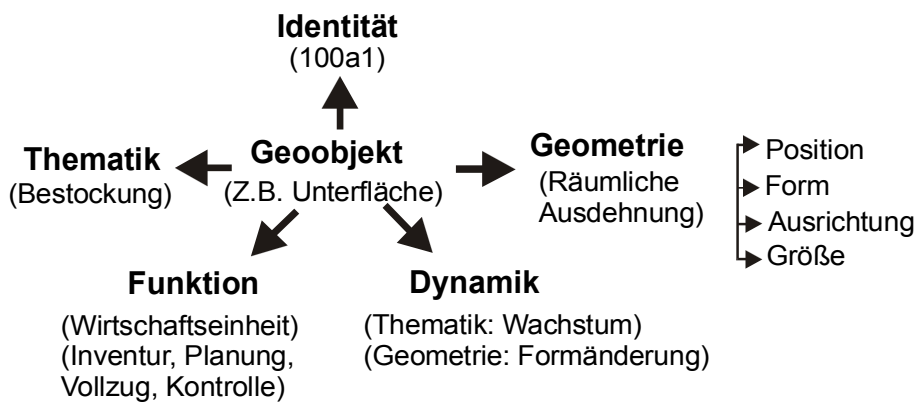

Abb. 10: Komponenten eines Geoobjektes.

Ein Geoobjekt wird von mehreren Komponenten gebildet (Abbildung 10):

Die inhaltliche Komponente (Thematik) stellt die inhaltlichen, semantischen Eigenschaften eines Geoobjektes dar, die auch Attribute genannt werden.

Die geometrische Komponente (Geometrie) beschreibt den Raumbezug (Position und Ausdehnung) eines Geoobjektes mit Hilfe eines räumlichen Bezugssystems. Geoobjekte ohne Ausdehnung sind ausschließlich durch ihre Position charakterisiert. Die ausgedehnten Objekte sind durch mehrere Eckpunkte und durch eine Funktion charakterisiert, welche die Verbindung 
der Punkte beschreibt. Solche Objekte besitzen außer der Position zusätzlich noch Form und Ausrichtung. Die Position ist die Lage der Punkte, die an der Bildung eines Geoobjektes beteiligt sind. Aus der absoluten Position, die durch Koordinaten beschrieben wird, gehen praktisch alle räumlichen Eigenschaften (Länge, Inhalt usw.) hervor.

Die Dynamik eines Geoobjektes umfasst seine zeitlichen Veränderungen. Darunter werden die Veränderungen des Geoobjektes bezüglich aller Komponenten aufgefasst. Die kartographische Komponente beschreibt die Art und Weise der kartographischen Abbildung. Rolle, Funktion und Verhalten beschreiben die Aufgabe eines Geoobjektes und die Methoden, die an ihm angewendet werden können.

Die Beziehungen (Relationen) beschreiben das Verhältnis zwischen den Objekten. Sie sind keine individuellen Objekteigenschaften, sondern verbinden immer zwei oder mehrere Objekte. Die räumlichen Beziehungen beschreiben die relative Lage der Geoobjekte zueinander. Man unterscheidet die Beziehungen angesichts der topologischen Eigenschaften (topologische Beziehungen), der Richtung (Richtungsbeziehungen) und der Entfernung (Entfernungsbeziehungen). Die hierarchischen Beziehungen (oder allgemeiner Ordnungsbeziehungen) betrachten Beziehungen zwischen Objekten untereinander und Objekten und Objektklassen in verschiedenen Hierarchieebenen. Die zeitlichen Beziehungen stellen das Verhältnis der zeitlichen Versionen der Geoobjekte, oder die Verhältnisse zwischen den Ereignissen und Prozessen dar.

Die Dimension eines Geoobjektes charakterisiert die Anzahl der voneinander unabhängigen Richtungen des Raumes, die zur vollständigen geometrischen Beschreibung des Geoobjektes erforderlich sind (STREIT 2000). Der Dimensionsbegriff wird auch auf die topologische und thematische Modellierung von Geoobjekten übertragen. Die Eigenschaften des Geoobjekts können auf unterschiedlicher Maßskala (Nominal, Ordinal, Intervall, Ratio) gemessen werden. Die Differenz der Dimension eines Geoobjektes und des ihm zugrundeliegenden Raumes wird als co-dimension bezeichnet.

Die Attributwerte von Geoobjekten können sich mit der Lage der Geoobjekte ändern. Die Geoobjekte besitzen dann hinsichtlich dieser Eigenschaft eine räumliche Varianz. Diese räumliche Varianz wird auch als räumlicher Prozess bezeichnet. Will man auf die zeitliche Variabilität räumlich veränderlicher 'Prozesse' besonders hinweisen, so spricht man von dynamischen räumlichen Prozessen (STREIT 2000).

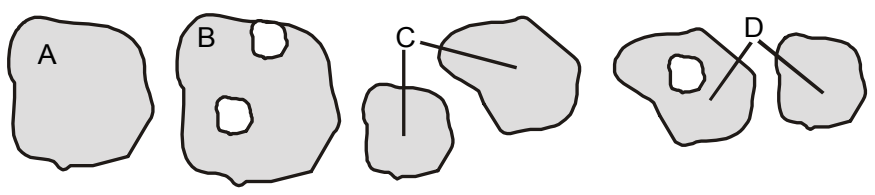

Abb. 11: Beispiele komplexer Geometrie eines Geoobjektes: (A) einfaches Polygon, (B) Polygon mit Löchern, (C) zusammengesetztes Polygon, (D) zusammengesetztes Polygon mit Löchern.

Wird eine Zuordnungsvorschrift benutzt, die mehrere Geoobjekte zu einem Geoobjekt oder einer Klasse oder Hierarchieebene zusammenfasst, um ein Phänomen höherer Ordnung zu konstruieren, entstehen komplexe Geoobjekte (Abbildung 11). Das Ergebnis dieser Vorschrift entspricht einem zusammengesetzten Geoobjekt (Aggregation) oder einer Klasse (Menge) der 
Geoobjekte. Wird diese Vorschrift für eine Klasse angewendet, ist das Ergebnis wiederum eine (Super- bzw. Sub-) Klasse (Generalisieren, Differenzieren). Bei einfachen Geoobjekten bezieht sich die inhaltliche Komponente auf die gesamte räumliche Komponente, bei den komplexen jeweils nur auf einige Teile (MAERKER 1998).

\subsubsection{Geometrische Eigenschaften eines Geoobjektes}

Die Beschreibung eines komplexen geometrischen Gebildes ist äußerst schwierig. Die morphologischen Eigenschaften eines Geoobjektes umfassen Form, Größe und Ausrichtung. Die Größe betrifft den Inhalt und den Umfang der Geoobjekte. Die Form beschreibt die geometrische Figur eines Geoobjektes. Die geometrischen Formen sind Äquivalenzklassen der geometrischen Individuen, die durch eine Transformation ineinander überführt werden können. Die Ausrichtung ist eine Eigenschaft der Form, die mit Hilfe eines Bezugsrahmens bestimmt werden kann.

Zur Bildung der Äquivalenzklassen komplexer Geometrien wurden mehrere Ansätze entwickelt. Durch die Vereinfachung der Geometrie durch Approximation und Zerlegung wird eine ursprünglich komplexe und schwer handhabbare Geometrie durch eine oder mehrere simple Geometrien ersetzt. Bei den Invarianzansätzen werden die Aspekte der Geometrie erfasst, die bei einer Transformation invariant bleiben. Bei der Abstraktion werden bedeutsame messbare Eigenschaften der Geometrie extrahiert und miteinander in Form einer Messzahl kombiniert.

Die Approximation versucht, eine komplexe Geometrie durch eine annährend gleiche oder ähnliche aber einfachere $\mathrm{zu}$ ersetzen, die jedoch alle problemrelevanten Eigenschaften der ursprünglichen Geometrie behält. In Geo-Datenbanksystemen ist sie für eine effiziente Anfragebearbeitung ein unverzichtbares Konzept. Sie unterstützt den selektiven Suchprozess durch eine Vereinfachung der Komplexität der Objektgeometrie.

Ein Geoobjekt kann durch konservative, progressive oder generalisierende Approximation vereinfacht werden (Abbildung 12). Bei der ersten liegt das Geoobjekt vollkommen in der approximativen Geometrie. Die progressive Approximation (auch Kernel-Approximation) liegt vollkommen in dem Geoobjekt. Bei der generalisierenden Approximation wird die Objektkontur vereinfacht. Die Approximation kann aus einem Element oder aus mehreren disjunkten oder sich überlappenden Elementen bestehen.

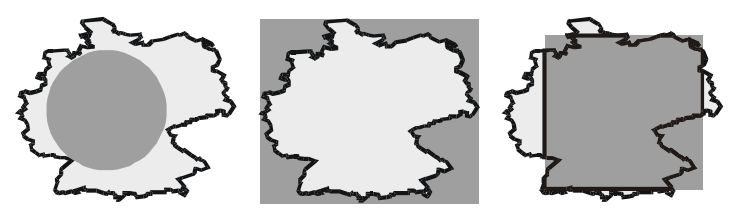

Abb. 12: Progressive, konservative und generalisierende Approximation eines Geoobjektes.

Eine der meist verbreiteten Approximationen ist die BB (bounding box) Approximation. BB ist das kleinste objektumschließende, achsenparallele Rechteck, das durch vier Parameter (die Koordinaten der gegenüberliegenden Eckpunkte oder die Koordinaten des Zentrums und die Ausdehnung in $x$ und $y$ Richtung) bestimmt ist. Die BB Approximation ist translationsinvariant, eindeutig und nicht rotationsinvariant. 
Weitere Approximationen sind die Kreis- oder Ellipsen- Approximationen. Vertreter der Approximation durch nicht primitive-Geoobjekte sind die konvexe Hülle, das minimal umgebende n-Eck oder die Z-Wert Approximation. Bei der letzteren wird der Objektraum als Folge von orthogonalen, äquidistanten Gittern mit immer feineren Unterteilungen betrachtet. Durch rekursives Halbieren des Objektraumes abwechselnd in x- und y-Richtung ermittelt der Approximationsalgorithmus diejenige Zelle eines Gitters, die das Objekt vollständig umgibt.

Ein typisches Beispiel einer konservativen mengenorientierten Approximation ist die Quadtree Approximation. Der Raum wird mit Hilfe eines sukzessiven Unterteilens in vier gleich große Quadranten (Zellen) zerlegt. Die Zellen, die nicht vollständig im Objekt oder nicht vollständig außerhalb des Objekts liegen, werden weiter unterteilt. Die Unterteilung wird bei Erreichung eines vorgegebenen Wertes der maximalen Auflösung abgebrochen. Die Zellen, die vollständig im Objekt liegen, oder dieses teilweise überdecken, bilden die Approximation.

Aus progressiven Approximationen ist der Kern von Polygonen wohl bekannt. Er wird durch eine Menge von Punkten gebildet, die vom gesamten Rand des Polygons sichtbar sind. Für jeden Punkt des Randes existiert also eine Verbindungstrecke zu jedem Punkt des Kernes, die nicht aus dem Objekt rausgeht. Weitere Approximationen dieser Art sind der maximal eingeschlossene Kreis (MEK) und das Rechteck.

Die Zerlegung entspricht dem algorithmischen Prinzip 'Divide and Conquer' (SCHNEIDER, R. 1993). Es wird dabei Zeit und Kapazität in die Vorbereitung investiert, die bei der Bearbeitung zurück gewonnen wird. Die Zerlegung wird beispielweise in der Computational geometry und in der Datenbanktheorie verwendet, um Algorithmen und Tests zu vereinfachen und sie dadurch schneller ausführbar zu machen. Wir betrachten einen anderen Aspekt der Zerlegung. Ein Geoobjekt mit Ausdehnung wird in diesem Ansatz zerlegt, um es nach seinen einzelnen Komponenten zu bewerten.

Die Zerlegungstechniken können nach mehreren Aspekten gegliedert werden. Wird der ganze (Konfigurations)Raum, in dem die Geoobjekte eingebettet sind, in disjunkte Zellen zerlegt, deren Vereinigung die ganze Menge der Geoobjekte überdeckt, spricht man von Raumzerlegung. Eine Zelle enthält dann den Teil eines oder mehrerer Geoobjekte. Im Gegensatz dazu steht die Objektzerlegung, bei der jedes Geoobjekt unabhängig von anderen in mehrere Teile zerlegt wird.

Orientiert sich eine Zerlegungstechnik an einem Verfahren, das vorgegeben wird, bezeichnet man sie auch als verfahrensorientierte Zerlegungen. Eine komponentenorientierte Zerlegung teilt ein Geoobjekt in wohldefinierte kleinere Komponenten. Sind die Teilkomponenten disjunkt, spricht man auch von Partitionierung. Überlappen sich die Teilkomponenten, bezeichnet man die Zerlegung als Covering. Schließlich kann eine Zerlegung ohne oder mit Verwendung zusätzlicher Punkte durchgeführt werden. Heterogene Zerlegungen erzeugen Teilkomponenten von verschiedenen Typen, Homogene nur von einem Typ.

Wohl bekannte und verbreitete Techniken sind die Quadtree-Zerlegungen. Sie können sowohl für mehrere Geoobjekte, als auch für ein einziges Geoobjekt verwendet werden. Der Konfigurationsraum oder das Geoobjekt wird dabei durch ein festes, orthogonales Gitter partitioniert. Die Elemente der Zerlegung werden Zellen genannt. Es gibt eine ganze Fülle an Zerlegungsverfahren in der Computational geometry. Der Typ der Teilkomponenten kann beispielweise Dreieck, Rechteck, Trapez, konvexes, sternförmiges oder spiralförmiges Polygon 
sein. Das meist verwendete Verfahren der Partitionierung ist die Triangulation. Die auf diese Weise ohne Verwendung der Steinerpunkte erzeugten Dreiecke sind paarweise disjunkt. Die Delaunay Triangulation trianguliert lediglich eine konvexe Hülle einer Punktmenge. Sie verkörpert das duale Problem des Voronoi-Diagramms. Eine ihrer Varianten, bezeichnet auch als Constrained-Delaunay-Triangulation, stellt eine Verallgemeinerung der Triangulation von Polygonen mit Löchern dar (SCHNEIDER, R. 1993). Dabei werden die Eckpunkte verwendet und die Kanten berücksichtigt. Trapeze stellen eine Klasse konvexer Vierecke dar, bei denen zwei der vier Seiten zueinander parallel verlaufen. Eine einfache Trapez-Zerlegung erzielt man, indem man $\mathrm{zu}$ jedem Eckpunkt horizontale Verbindungslinien erzeugt, die innerhalb des Polygons liegen und zur nächsten Kante verlaufen.

Bei der Abstraktion wird eine komplexe Geometrie durch einige ihrer qualitativen oder quantitativen Eigenschaften beschrieben. Äquivalenzklassen werden mit Hilfe der Ähnlichkeit der abstrahierten Eigenschaften definiert. Diese Methode entspricht einem sog. Feature-basierten Ansatz. Dabei wird eine Menge der Eigenschaften von Geoobjekten extrahiert und in einen Feature-Vektor umgewandelt. Der mit einer geeigneten Entfernungsfunktion ermittelte Abstand zwischen zwei Geoobjekten in diesem Feature-Raum beschreibt die Ähnlichkeit der Geoobjekte. Dieser Ansatz liegt vielen Data mining Verfahren zugrunde.

In verschiedenen Disziplinen wurden zahlreiche Indizes entwickelt, die eine komplexe Geometrie abstrahieren. Der Schwerpunkt liegt bis jetzt trotzt wachsender Kritik bei den quantitativen Messzahlen. Ihre Schwäche liegt in ihrer beschränkten Diskriminierungsfähigkeit. Die Auswahl und Gewichtung der Parameter lässt sich folglich nicht einfach begründen.

Die Indizes müssen einige Voraussetzungen erfüllen. Sie müssen beispielsweise gleiche Werte für gleiche oder ähnliche Strukturen liefern. Ihre Spreitung soll den gesamten potentiellen Wertebereich aufweisen. Sie sollen Informationen umfassen, die nicht von anderen Indizes geliefert werden und ihre Werte sollen unabhängig von der absoluten Flächengröße sein (vgl. O'NEILL, zitiert nach TRAUB \& KLEINN 1997).

Als Eingangsgröße der Messzahlen kann eine beliebige räumliche Eigenschaft angenommen werden. Dabei ist man jedoch auf messbare Größen angewiesen (TrAUB \& KLEINN 1997). Zu diesen gehören beispielweise Fläche, Umfang, Randlinienlänge und Randflächenanteil, fraktale Dimension usw. Die Aggregation einzelner Messzahlen in zusammengesetzte Indizes (wie beispielsweise Fourierformmesszahlen) ist durch steigende Komplexität gekennzeichnet. Die Erkennung und Anpassung einer Form sind mögliche Anwendungen von diesen Vorgängen.

In der quantitativen Landschaftsökologie wurde eine ganze Reihe quantitativer Indizes entwickelt. Der Schwerpunkt liegt bei Konzepten, die auf Umfang und Fläche basieren. Bei gegebener Fläche kann der Umfang, abhängig von der Komplexität der Umfangslinie oder der Form der Figur, stark variieren. Drei hierarchische Stufen werden unterschieden: Patch (Geoobjekt), Klasse (Class, Menge der Geoobjekte mit gleicher Eigenschaft) und Landschaft (Landscape, die Gesamtheit der Geoobjekte). Die entwickelten Indizes werden in folgende Klassen eingeteilt:

- Flächenindizes (area metrics)

- Kantenindizes (edge metrics)

- Formindizes (shape metrics)

- Kernflächeindizes (core area metrics) 
- Nächste-Nachbarn Indizes (nearest-neighbor metrics)

- Diversitätsindizes (diversity metrics)

In FRAGSTAT und auch in einigen GIS Programmen wie ARCINFO (FRAGSTAT\&ARC), ARCVIEW (PatchAnalyst) oder IDRISI wurden einige solche Messzahlen implementiert.

Invarianzansätze untersuchen die geometrischen Eigenschaften der Geoobjekte mit Hilfe von geometrischen Transformationen. Dabei können Eigenschaften erfasst werden, die bei einer bestimmten Gruppe von Transformationen unverändert (invariant) bleiben (vgl. BITTNER \& FrANK 1998, CLEMENTINI \& Di FeliCE 1997A). Die Transformationsgruppe definiert in einem geometrischen Raum eine Äquivalenzrelation in der Gesamtheit der Figuren und damit Klassen zueinander äquivalenter Figuren bzw. invarianter Eigenschaften. Eine Transformationsinvariante kann sowohl qualitativer als auch quantitativer Natur sein. Im hierarchischen Aufbau der Geometrie kommen die qualitativen Invarianten im oberen, die quantitativen im unteren Teil vor: "Topology matters, metric refines" (EGENHOFER \& MARK 1995B).

Von Clementini \& Di Felice (1997a) wurde ein Modell der Formbeschreibung entwickelt, das die topologischen, projektiven und metrischen Eigenschaften in drei orthogonalen Dimensionen modelliert. Die Orthogonalität ist erst mit der Annahme erreicht, dass die eigentlich hierarchischen Eigenschaften immer nur diejenigen Merkmale besitzen, die nicht schon mit einer übergeordneten Eigenschaft erfasst wurden. Die metrische Achse enthält also nur die Eigenschaften, die nicht projektiv oder topologisch sind, und die projektive Achse nur die Eigenschaften, die nicht topologisch sind. Die Fortbewegung entlang der Achse führt zu höheren Granularitätsstufen. Sie beschreiben die Mächtigkeit der Domäne einzelner Eigenschaften. Die Granularitätstufen sind entlang der Achse geordnet. Beispiele der topologischen Eigenschaften können der Zusammenhang, die Anzahl der Komponenten und die Präsenz oder Abwesenheit von Löchern sein. Die projektiven Eigenschaften umfassen die Konzepte der Konvexität und Konkavität. Die metrischen Eigenschaften können mit Verlängerung (elongation), Symmetrie oder relativer Größe ausgedrückt werden.

\subsection{Räumliche Szene}

Eine räumliche Szene (RS) ist eine Menge von Geoobjekten, charakterisiert durch räumliche und a-räumliche Eigenschaften, in ihren räumlichen Beziehungen (BRUNS \& EGENHOFER 1996). Die ganzheitliche Betrachtung einer räumlichen Szene spiegelt sich in ihrer globalen Anordnung (Konfiguration) wider. Die zentrumsbezogene Betrachtung eines Ausschnittes einer räumlichen Szene wird durch lokale Anordnung beschrieben.

Eine räumliche Szene kann durch eine Konnektivitätsmatrix (connectivity matrix) oder durch einen Beziehungsgraph (relation graph) beschrieben werden (Abbildung 13). Die Konnektivitätsmatrix ist eine symmetrische Kreuztabelle von Objekten einer Szene, deren Zellen die Beziehungen der Elemente enthalten. Ein Beziehungsgraph ist ein Graph, deren Knoten die Geoobjekte und deren Kanten die Beziehungen darstellen. 
a)

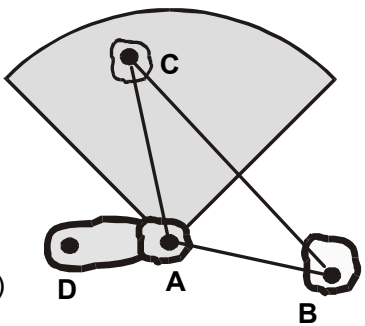

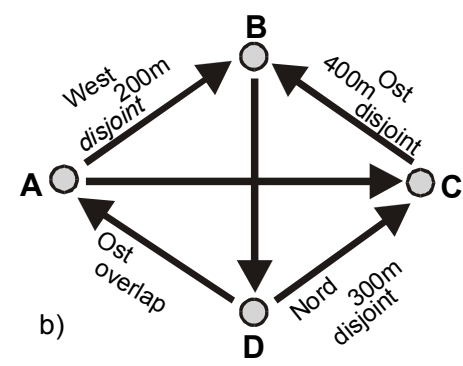

\begin{tabular}{|c|c|c|c|c|}
\hline & $\mathbf{A}$ & $\mathbf{B}$ & $\mathbf{C}$ & $\mathbf{D}$ \\
\hline $\mathbf{A}$ & $\mathrm{r}_{\mathrm{AA}}$ & $\mathrm{r}_{\mathrm{AB}}$ & $\mathrm{r}_{\mathrm{AC}}$ & $\mathrm{r}_{\mathrm{AD}}$ \\
\hline $\mathbf{B}$ & $\mathrm{r}_{\mathrm{BA}}$ & $\mathrm{r}_{\mathrm{BB}}$ & $\mathrm{r}_{\mathrm{BC}}$ & $\mathrm{r}_{\mathrm{BD}}$ \\
\hline $\mathbf{C}$ & $\mathrm{r}_{\mathrm{AB}}$ & $\mathrm{r}_{\mathrm{AB}}$ & $\mathrm{r}_{\mathrm{AB}}$ & $\mathrm{r}_{\mathrm{AB}}$ \\
\hline $\mathbf{D}$ & $\mathrm{r}_{\mathrm{DA}}$ & $\mathrm{r}_{\mathrm{DB}}$ & $\mathrm{r}_{\mathrm{DC}}$ & $\mathrm{r}_{\mathrm{DD}}$ \\
\hline
\end{tabular}

c)

Abb. 13: Darstellung einer räumlichen Szene: (a) räumliche Repräsentation, (b) Beziehungsgraph, (c) Beziehungsmatrix.

Eine räumliche Szene kann entweder durch ihre Beziehungen oder durch die Eigenschaften ihrer Objekte oder durch die Kombination beider beschrieben werden.

Eine RS kann ähnlich wie einzelne Geoobjekte nach dem Invarianzansatz beschrieben werden. Zwei räumliche Szenen sind als gleich anzusehen, wenn es eine Transformation gibt, mit der sie ineinander überführt werden können, ohne dass sich ihre Beziehungen ändern. Dieses Konzept definiert eine Äquivalenzrelation der räumlichen Szenen. Durch eine Erweiterung dieses Ansatzes um die konzeptionelle Nachbarschaft der Beziehungen kann auch eine Ähnlichkeit zweier räumlicher Szenen bestimmt werden.
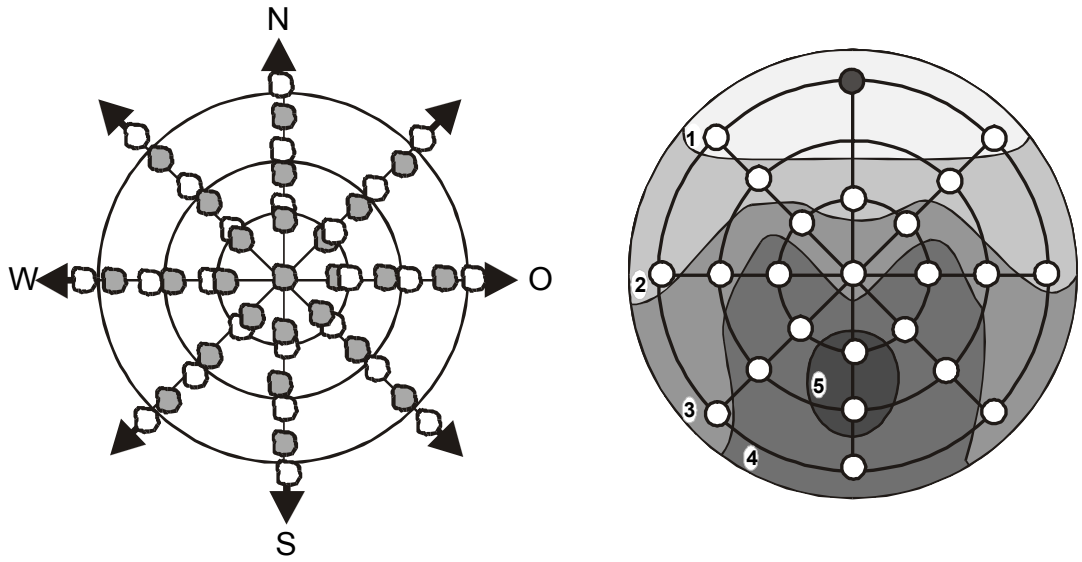

Abb. 14: Ähnlichkeit der räumlichen Beziehungen: a) schematische Darstellung unterschiedlicher Beziehungsarten, b) konzeptionelle Nachbarschaft der Beziehungen (In Anlehnung an BRUNS \& EGENHOFER 1996).

Zwei Relationen sind konzeptionelle Nachbarn, wenn sie ineinander durch eine graduelle Deformation (Änderung) überführt werden können, ohne dass während der Änderung eine andere Beziehung vorkommt (Abbildung 14). Die Ähnlichkeit ist ein Konzept, das die Abweichung von der Gleichheit (Äquivalenz) misst. Zwei räumliche Szenen sind desto ähnlicher, je kleiner die Deformation ist, die sie trennt (BRUNS \& EGENHOFER 1995). Für zwei räumliche Szenen mit gleicher Anzahl räumlicher Objekte existiert eine minimale Menge der graduellen Deformationen (Änderungen), die eine Szene in die andere transformiert. Die Anzahl der kontinuierlichen Änderungen ist proportional der Ähnlichkeit beider Szenen. Die Unähnlichkeit kann durch die Anzahl der unterschiedlichen Beziehungen und den Grad ihrer Verschiedenheit bestimmt werden 
(BRUNS \& EGENHOFER 1995). Andere Ansätze der Ähnlichkeit räumlicher Szenen umfassen Muster (pattern) und Verteilung ihrer Geoobjekte.

Die objektbezogene Szenenbeschreibung betrachtet eine räumliche Szene als Menge der Objekte (Geoobjektmenge) mit ihren individuellen Eigenschaften. Eine Geoobjektmenge kann durch die Verteilung aller individuellen Eigenschaften ihrer Elemente beschrieben werden. Darüber hinaus können neue mengenspezifische Eigenschaften verwendet werden. Von SCHNEIDER, R. (1993) werden die Eigenschaften von Objektmengen folgend zusammengefasst:

- Mengenspezifische Parameter (Anzahl der Objekte, Überlappung)

- Strukturparameter (Anzahl der Eckpunkte, Fläche, Umfang)

- Formparameter (Shape-Faktor, Rectangular-Shape-Faktor, Quiescence)

- Lageparameter (Ausrichtung, Verteilung)

Die Kombination einer beziehungsbezogenen und einer objektbezogenen Szenebeschreibung verwendet sowohl die individuellen Eigenschaften der Geoobjekte als auch ihre Beziehungen. Dies kann sowohl in der globalen als auch in der lokalen Konfiguration erfolgen.

Die Konsistenz einer räumlichen Szene lässt sich mit den Eigenschaften und Bedingungen der räumlichen Relationen bestimmen. Die Eigenschaften sind Gegenstand einer Algebra an Relationen (EGENHOFER 1994a und 1994b).

Konsistenz einer räumlichen Szene bedeutet, dass es keinen logischen Wiederspruch zwischen den räumlichen Beziehungen ihrer Beschreibung (constraints) gibt. Ein constraint network besteht aus einer Menge von Knoten (node), von orientierten und beschrifteten Kanten (directed edges), die jeweils zwei Knoten verbinden, und einer Menge von Regeln über die Semantik der Beschriftungen (labels) und ihrer Kombination (Abbildung 15). Die Aufgabe eines constraint network ist zu überprüfen, ob eine Menge von Beziehungen gleichzeitig erfüllt werden kann.
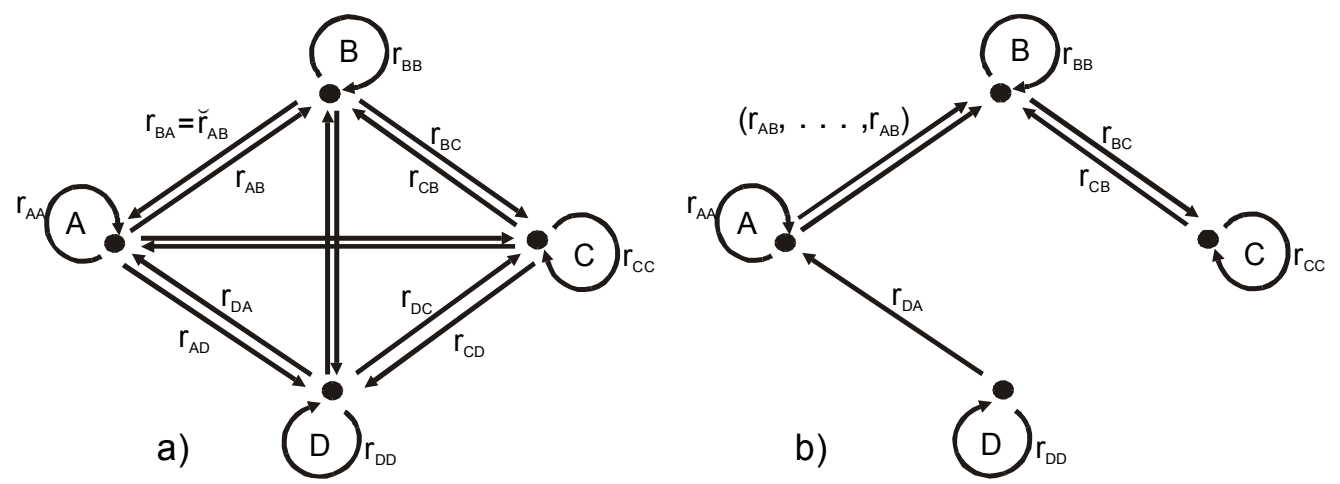

Abb. 15: Beispiele der Szenebeschreibung: (a) komplettes constraint network, (b) unkomplettes constraint network (in Anlehnung an EGENHOFER \& SHARMA 1993B).

Ein Pfad (path) ist eine Verbindung von zwei Knoten über Kanten mit gleicher Orientierung. In der räumlichen Repräsentation stellt jeder Knoten ein Geoobjekt und jede Kante eine räumliche Beziehung dar. Drei Bedingungen können für ein konsistentes Netz aufgestellt werden:

- node consistency: Die Beziehung eines Knotens mit sich selbst muss eine Identitätsrelation sein: $r_{\mathrm{AA}}=$ equal 
- arc consistency: Die entgegengesetzten Beziehungen zwischen zwei Geoobjekten müssen invers sein. $r_{A B}=r^{6}{ }_{B A}$

- path consistency: Die Relationen, die über alle möglichen Kompositionen abgeleitet werden können, dürfen sich nicht widersprechen.

$\mathrm{r}_{\mathrm{AB}}=\left(\mathrm{r}_{\mathrm{A} 1} ; \mathrm{r}_{1 \mathrm{~B}}\right) \cap\left(\mathrm{r}_{\mathrm{A} 2} ; \mathrm{r}_{2 \mathrm{~B}}\right) \cap \ldots \cap\left(\mathrm{r}_{\mathrm{An}} ; \mathrm{r}_{\mathrm{nB}}\right)$

Ein Netzwerk ist konsistent, wenn alle Kompositionen der Pfade mit der Länge 2 konsistent sind.

\subsection{Theorie der räumlichen Beziehungen}

"One of the fundamental concepts necessary for the analysis of spatial data in Geographic Information System is a formal understanding of the geometric relationship among arbitrary spatial objects" (EGENHOFER \& HERRING 1990).

Die Theorie der räumlichen Beziehungen befasst sich mit der Formulierung und Formalisierung von Konzepten der räumlichen Beziehungen. Die räumlichen Beziehungen beschreiben die relative Lage der Geoobjekte zueinander. Bei ihrer Formulierung spielen kognitive, linguistische und psychologische Aspekte eine wichtige Rolle.

Die Theorie der räumlichen Beziehungen konzentriert sich auf folgende Fragen:

- Welche geometrischen Eigenschaften der Geoobjekte beschreiben ihre gegenseitigen räumlichen Beziehungen ?

- Wie können die Beziehungen mit Hilfe von geometrischen Eigenschaften formal definiert werden?

- Welche ist die minimale Menge der räumlichen Beziehungen?

- Wie können Beziehungen verknüpft (relation algebra), angeordnet (räumliche Ähnlichkeit) und quantifiziert (metrische Aspekte der Beziehungen) werden?

Die räumlichen Relationen stehen durch eine graduelle Deformation in einer Nachbarschaftsbeziehung zueinander. Die Relation $r_{n}$ geht unter einer kontinuierlichen Deformation in Relation $r_{m}$ über. Die Nachbarschaftsbeziehungen werden mit Hilfe sog. konzeptioneller Nachbarschaftsgraphen (conceptual-neighborhood graph) dargestellt (Abbildung 14, 17). Sie stellen eine Ordnung der Relationsmenge (BRUNS \& EGENHOFER 1990) dar. Das Konzept der graduellen Änderung ist für die topologischen Relationen, aber auch für die kardinale Richtung und für die approximative Entfernung anwendbar. Zwei räumliche Relationen, die nur eine kleine Deformation trennt, sind ähnlicher als diejenigen mir größerer Deformationstrennung. Je kleiner die Beziehungsentfernung ist, desto kleiner ist die konzeptionelle Differenz zwischen zwei Relationen.

Neben den quantitativen Beziehungen gewinnen die qualitativen räumlichen Beziehungen zunehmend an Bedeutung. Sie sind der menschlichen Wahrnehmung näher. Die qualitativen Beziehungen basieren auf einer kleinen Menge von Symbolen und der Menge der Schlussregeln für die Kombination dieser Symbole. Die Symbole sind entweder linear (Entfernung) oder zyklisch (Richtung) geordnet. Sie beschreiben die gleiche Domäne wie die quantitativen Beziehungen, nur ihre Bezeichnung ist unterschiedlich (HoNG et al. 1995). Eine qualitative 
Relation entspricht einem Intervall quantitativer Relationen, und eine quantitative Relation soll genau einer qualitativen entsprechen (intervallbasierte Transformation).

Die qualitative Beziehung ist inhärent unsicher. Ihre Bedeutung entspricht aber gerade wegen dieser Eigenschaft eher der menschlichen Beobachtung und Schlussfolgerung (HoNG et al. 1995). "Although qualitative relations are often vague in their geometric meaning and have less resolution than their quantitative counterpart, people have little difficulty in processing them and using them to communicate with others." (GOYAL \& EGENHOFER 1997).

Die räumliche Schlussfolgerung (spatial reasoning) basiert auf dem Prinzip der Komposition von bekannten Beziehungen zur Ableitung unbekannter Beziehungen. Eine Komposition besteht aus dem Kompositionsoperator (;) und zwei bekannten Relationen: r(A,B) ; r(B,C), die ein Objekt (B) gemeinsam haben, sowie der abzuleitenden Beziehung r(A,C).

Das Design der räumlichen Schlussfolgerung muss von einer eindeutigen Definition der räumlichen Beziehungen und Operationen ausgehen (HONG et al. 1995). Die Suche nach bestimmten Kombinationen der Relationen muss schnell zur Antwort führen. Vor der Suche nach der Antwort soll das System überprüfen können, ob die vorhandenen Informationen ausreichend sind, um die gesuchte Antwort zu finden. Die Anzahl der möglichen Antworten soll möglichst gering gehalten werden. Eine Schlussfolgerung qualitativer Beziehungen kann entweder diese in ein quantitatives Format überführen und mit Hilfe von quantitativen Methoden lösen, oder sie in qualitativem Format lösen.

Die künftigen GIS sollen nicht nur einen Mechanismus für die effektive Speicherung und die Anfragebearbeitung räumlicher Daten, sondern auch ein intelligentes wissensbasiertes System darstellen, das menschliches Expertenwissen zur Entscheidungsfindung verwendet.

\subsubsection{Mengenbeziehungen}

Die Mengenbeziehungen bilden den Ausgangspunkt für die Definition der topologischen und der Ordnungsbeziehungen. Das grundlegende Konzept ist Enthaltensein (containment). GÜTING (1988) beschreibt Beziehungen, die sich auf die Mengenoperationen stützen:

$\mathrm{x}=\mathrm{y}:=$ points $(\mathrm{x})=$ points $(\mathrm{y})$

$\mathrm{x} \neq \mathrm{y}:=$ points $(\mathrm{x}) \neq$ points $(\mathrm{y})$

$\mathrm{x}$ inside $\mathrm{y}:=$ points $(\mathrm{x}) \subseteq$ points $(\mathrm{y})$

$\mathrm{x}$ outside $\mathrm{y}:=$ points $(\mathrm{x}) \cap$ points $(\mathrm{y})=\varnothing$

$\mathrm{x}$ intersect $\mathrm{y}:=$ points $(\mathrm{x}) \cap$ points $(\mathrm{y}) \neq \varnothing$

Die Beziehungen sind nicht komplett und auch nicht eindeutig (= und inside sind gleichzeitig intersect). Der Nachteil dieses Ansatzes liegt vor allem an seiner Unfähigkeit, einzelne Teile der Objekte in Betracht zu ziehen. Weitere Ansätze, die gefolgt sind (PULlar 1988, WAGER 1988, zitiert nach EGENHOFER \& FRANZOSA 1990) sind aus diesem Prinzip hervorgegangen.

\subsubsection{Topologische Beziehungen}

Die Topologie ist ein qualitatives Konzept, das völlig unabhängig von quantitativen Größen ist. Die topologischen Beziehungen sind räumliche Beziehungen, die unter Transformationen wie Translation, Skalierung oder Rotierung erhalten bleiben. Sie werden durch die Konzepte der 
Nachbarschaft (adjacency), des Enthaltenseins (containment) und Überschneidens (intersection) beschrieben.

Aus der topologischen Sicht besteht ein (geschlossenes) Geoobjekt A aus Objektinnerem $\mathrm{A}^{\circ}$ (interior, Innenbereich) und Rand $\partial \mathrm{A}$ (boundary, Objektrand, Grenzbereich). Beide Teile zusammen bilden die geschlossene Hülle von A (closure, Abschluß). Das Komplement eines Objektes im Raum bildet das Objektäußere $A^{-}$(exterior). Interior, boundary und exterior schließen sich gegenseitig aus.

$\mathrm{Zu}$ Beschreibung und Formalisierung der topologischen Beziehungen wurden mehrere Ansätze entwickelt: Punktmengenansatz, Modelle algebraisch-topologischer Gebilde, Logikansätze und Intervallbeziehungen.

\subsubsection{4 und 9 Intersection Modelle}

EGENHOFER \& FRANZOSA (1991), EGENHOFER \& HERING (1990) haben zwei Relationsmodelle zur Beschreibung der topologischen Beziehungen entwickelt. Das 4-Intersection Modell basiert nur auf den Schnittmengen (intersection) vom Inneren und dem Rand. Aus der Kombination der Objektteile ergeben sich vier mögliche Intersectionmengen.

- boundary - boundary intersection

- interior - interior intersection

- boundary-interior intersection

- interior - boundary intersection

$$
I_{4}(A, B)=\left(\begin{array}{ll}
A^{\circ} \cap B^{\circ} & A^{\circ} \cap \partial B \\
\partial A \cap B^{\circ} & \partial A \cap \partial B
\end{array}\right)
$$

Zur Beschreibung der Schnittmengen können nur diejenigen Eigenschaften ausgewählt werden, die bei der Anwendung von topologischen Abbildungen (Homöomorphismus) invariant bleiben (topologische Invarianten). In dem Grundmodell wird die Inhaltsinvariante (content invariant) angewendet, welche nur leere und nicht-leere Schnittmenge unterscheidet.

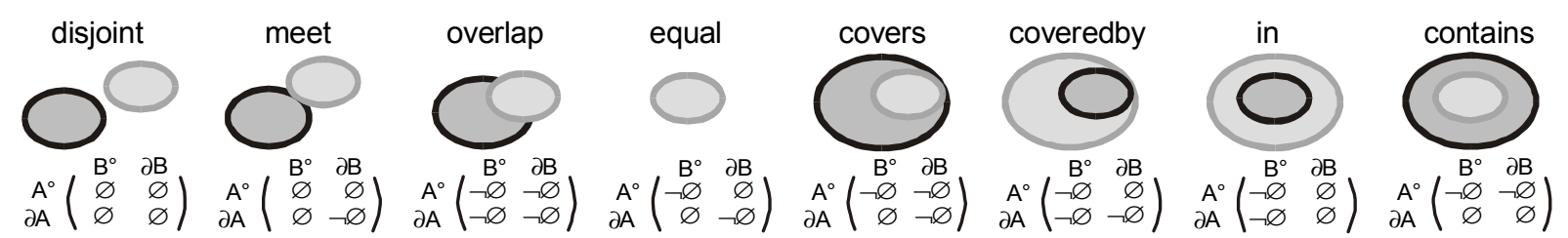

Abb. 16: Topologische Beziehungen zwischen Polygonen nach 4-Intersections Modell.

Bei zwei zulässigen Ausprägungen der Schnittmengenbeschreibung (Leer/Nicht leer) ergeben sich insgesamt $16\left(4^{2}\right)$ Beziehungen, die alle in $\mathbb{R}^{2}$ realisierbar sind und die sich gegenseitig ausschließen. 

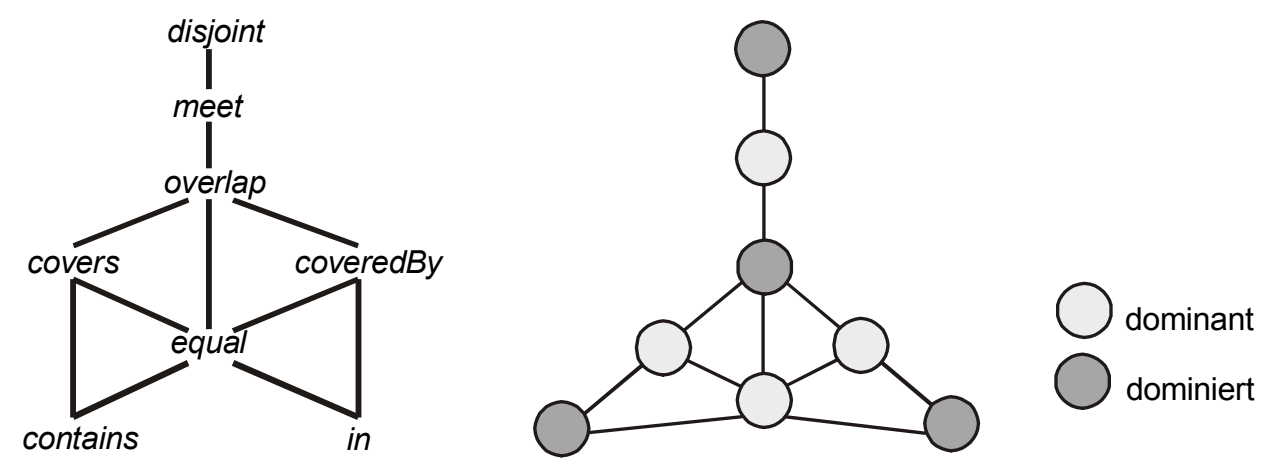

Abb. 17: Konzeptioneller Nachbarschaftsgraph der topologischen Beziehungen zwischen einfachen Polygonen.

Für die Polygon-Polygon ${ }^{4}$ Beziehungen in $I R^{2}$ und die Linie-Linie Beziehungen in $I R^{1}$ ergibt sich eine Menge von 8 möglichen (realisierbaren) topologischen Beziehungen (Abbildung 16, 17). Diese sind vollständig (die Objekte stehen immer in einer Beziehung aus dieser Menge zueinander) und eindeutig (das Auftreten einer Beziehung schließt alle anderen aus): disjoint, meets, overlap, equal, contains, inside, covers, coveredBy.

Das zweite Modell, auch 9-intersection Modell genannt, zieht außer dem Inneren $\left(\mathrm{A}^{\circ}\right)$ und dem Rand (ӘA) auch das Äußere $\left(A^{-}\right)$in Betracht. Aus der $3 \times 3$ Matrix ergeben sich $512\left(2^{9}\right)$ verschiedene 9-Intersektionsmatrizen. Die Anzahl der realisierbaren Beziehungen hängt von der Dimension des Raumes und des Geoobjektes sowie von den topologischen Eigenschaften der Geoobjekte ab. Die Differenz der Dimension des Raumes und des Geoobjektes wird auch als codimension bezeichnet.

$$
I_{9}=(A, B)=\left(\begin{array}{lll}
A^{\circ} \cap B^{\circ} & A^{\circ} \cap \partial B & A^{\circ} \cap B^{-} \\
\partial A \cap B^{\circ} & \partial A \cap \partial B & \partial A \cap B^{-} \\
A^{-} \cap B^{\circ} & A^{-} \cap \partial B & A^{-} \cap B^{-}
\end{array}\right)
$$

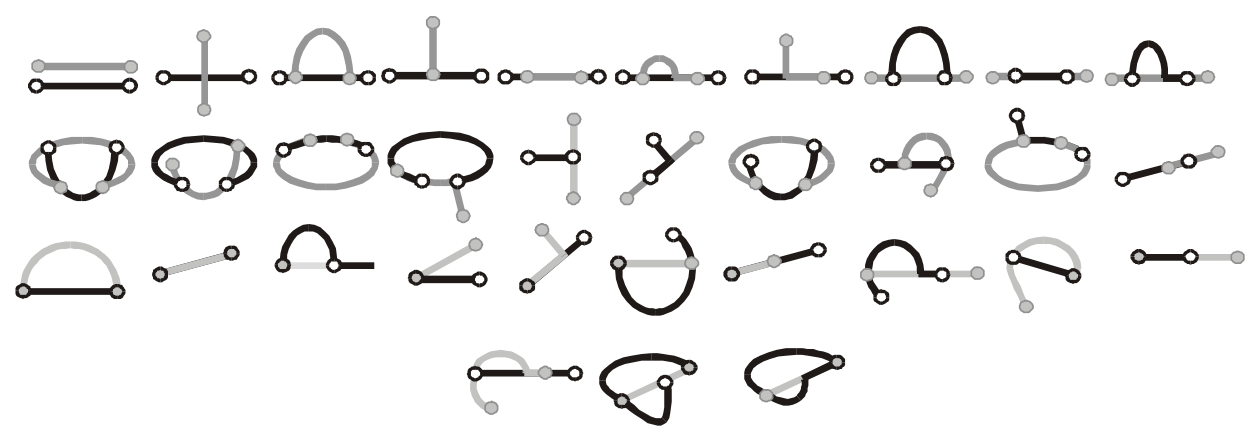

Abb. 18: Topologische Beziehungen zwischen Linien nach der Inhaltsinvariante.

Aus der 9-Intersectionmatrix ergeben sich die gleichen topologischen Beziehungen für zwei Regionen in $I R^{2}$ oder zwei Linien in $I R^{1}$, sie erlaubt aber mehrere Beziehungen in einem Raum höherer Dimension. Die 9-Intersectionmatrix bildet also eine Supermenge zur 4Intersectionmatrix.

${ }^{4}$ Wenn nicht anders erklärt, werden unter ,Polygon' immer einfache regurär geschlossene Polygone gemeint (in eng. Literatur auch region genannt) 

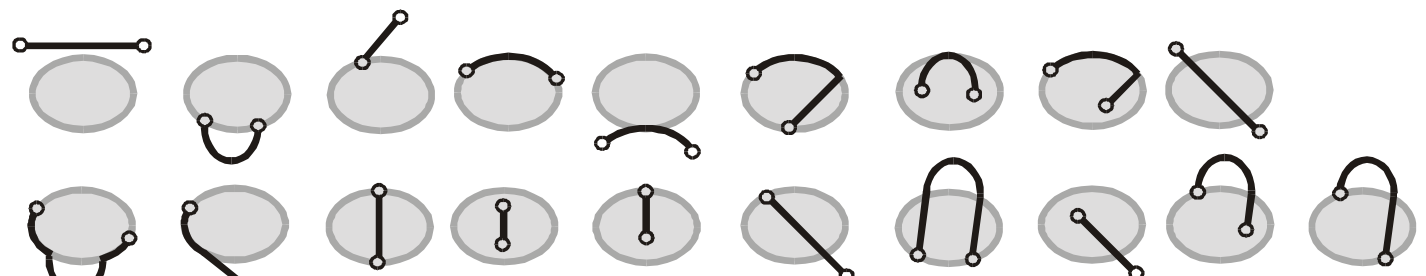

Abb. 19: Topologische Beziehungen zwischen einer Linie und einem Polygon.

Aus den 512 der 9-Intersectionmatrizen sind für zwei Polygonen nur 19 möglich, falls die Ränder nicht zusammenhängend sein müssen. Ist nur der zusammenhängende Rand zugelassen, sind nur 8 Beziehungen möglich. Von 57 Relationen zwischen zwei Linien sind 33 für einfache Linien (Abbildung 18) und 24 nur für komplexe Linien möglich. Die Anzahl der Beziehungen zwischen einer Linie und einer Region ist 20 (Abbildung 19). Eine aus diesen kommt nur dann vor, wenn die Linie komplex ist.

\subsubsection{Calculus based Modell}

Im calculus based Modell (CLEMENTINI et al. 1993) geht man vom Object-Calculus, einer formalen Anfragesprache der geographischen Datenbank, aus. Ein Faktum (fact) in dieser Sprache besteht aus dem Tripel $\left\langle\lambda_{1}, \mathrm{r}, \lambda_{2}\right\rangle$. Dieses besagt, dass zwei Geoobjekte $\lambda_{1}, \lambda_{2}$ beliebiger Dimension zueinander in Beziehung $r$ stehen.

Ähnlich wie im 4-Intersection Modell werden die Schnittmengen des Randes und des Inneren berücksichtigt, das Modell gilt aber für jedes geometrische Objekt und die Dimension der Schnittmenge wird explizit berücksichtigt (dimension extended method). Aus den 4 möglichen Dimensionen $(\varnothing, 0,1,2)$ der Schnittmengen ergeben sich insgesamt $4^{4}=256$ unterschiedliche Fälle. Nach der Beseitigung der unmöglichen Beziehungen bleiben insgesamt 52 realisierbare Fälle (Tabelle 3).

Tab. 3: Topologische Beziehungen des dimension extended Modells.

\begin{tabular}{|c|c|}
\hline Beziehung & Anzahl der Fälle \\
\hline Polygon/ Polygon & 9 \\
Linie / Polygon & 17 \\
Punkt / Polygon & 3 \\
Linie / Linie & 18 \\
Punkt / Linie & 3 \\
Punkt / Punkt & 2 \\
\hline$\Sigma$ & 52 \\
\hline
\end{tabular}

Alle 52 Beziehungen können mit Hilfe von den folgenden 5 beschrieben werden: $\{$ disjoint, touch, overlap, cross, in\}.

\subsubsection{RCC topologische Relationen}

Der RCC Ansatz (CUI et al. 1993) geht von einer punktlosen (pointless) Geometrie aus. Das einzige Geoobjekt, das in diesem Ansatz zugelassen wird, ist das Polygon (bzw. die Region). Das Modell baut auf der binären (dyadischen) Beziehung $\mathrm{C}(\mathrm{x}, \mathrm{y})$ auf, die besagt, dass ' $x$ connects with $y^{\prime}$. Diese Relation ist symmetrisch und reflexiv. Eine mögliche topologische Interpretation dieser Relation ist eine punktförmige Schnittmenge der abgeschlossenen Hüllen von zwei Geoobjekten. 
Mit Hilfe dieser Relation lässt sich eine Menge von topologischen Beziehungen definieren (Abbildung 20):

$\begin{array}{ll}\mathbf{D C}(\mathrm{x}, \mathrm{y}) & \mathbf{x} \text { is disconnected from } \mathbf{y} \\ \mathbf{P}(\mathrm{x}, \mathrm{y}) & \mathbf{x} \text { ist a part of } \mathbf{y} \\ \mathbf{P P}(\mathrm{x}, \mathrm{y}) & \mathbf{x} \text { is a proper part of } \mathbf{y} \\ \mathrm{x}=\mathrm{y} & \mathbf{x} \text { is identical with } \mathbf{y} \\ \mathbf{O}(\mathrm{x}, \mathrm{y}) & \mathbf{x} \text { overlaps } \mathbf{y} \\ \mathbf{D R}(\mathrm{x}, \mathrm{y}) & \mathbf{x} \text { is discrete from } \mathbf{y} \\ \mathbf{P O}(\mathrm{x}, \mathrm{y}) & \mathbf{x} \text { partially overlaps } \mathbf{y} \\ & \\ \mathbf{E C}(\mathrm{x}, \mathrm{y}) & \mathbf{x} \text { is externally connected with } \mathbf{y} \\ \mathbf{T P P}(\mathrm{x}, \mathrm{y}) & \mathbf{x} \text { is a tangential proper part of } \mathbf{y} \\ \mathbf{N T P P}(\mathrm{x}, \mathrm{y}) & \mathbf{x} \text { is a nontangential proper part of } \mathbf{y}\end{array}$

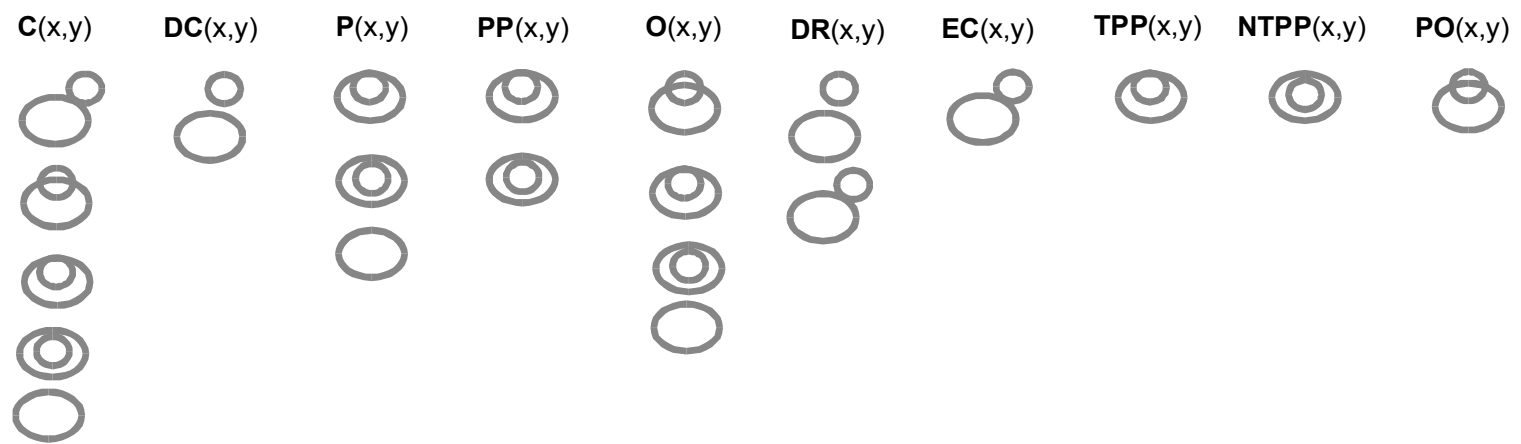

Abb. 20: Topologische Beziehungen nach dem RCC Ansatz.

Die Menge der Relationen $\Phi \in\{$ P,PP,TPP und NTPP $\}$ ist nicht symmetrisch, es gibt für sie eine Menge der inversen Beziehungen $\Phi^{-1}$. Die Menge der Relationen DC,EC,PO,=,TPP,NTPP, $\mathrm{TPP}^{-1}, \mathrm{NTPP}^{-1}$ ist vollständig und eindeutig (paarweise disjunkt).

\subsubsection{Modell der Grundfläche und der generischen Fläche}

Der Ansatz von WorBoys \& BOFAKOs (1993) geht von algebraischen Gebilden aus. Ein Atom (atom) ist eine Untermenge von $\mathbb{R}^{2}$, die topologisch äquivalent zur geschlossenen Kreisscheibe ist. Durch eine Aggregation der Atome entstehen zusammengesetzte Objekte. Zwei unterschiedliche Atome haben eine abzählbare Schnittmenge (die Inneren schneiden sich nicht) und die zusammengesetzten Objekte haben keine Löcher. Das Skelett (skeleton) einer endlichen Menge der Atome (A), bezeichnet auch als $\Sigma$ A, ist ein Graph, dessen Knoten die Atome von A sind. Ist die Schnittmenge von zwei Atomen nicht leer, werden entsprechende Knoten mit einer Kante verbunden. 


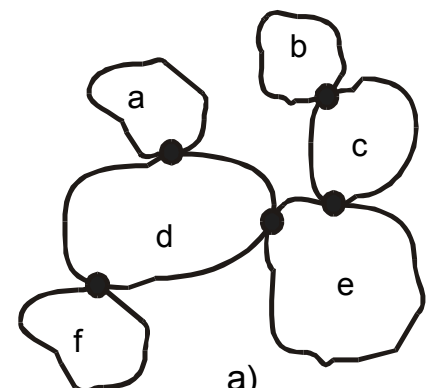

a)

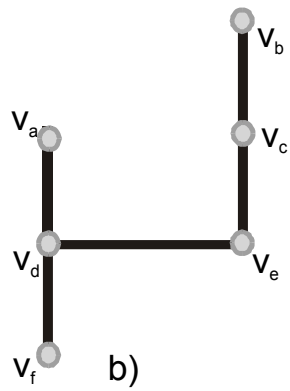

b)

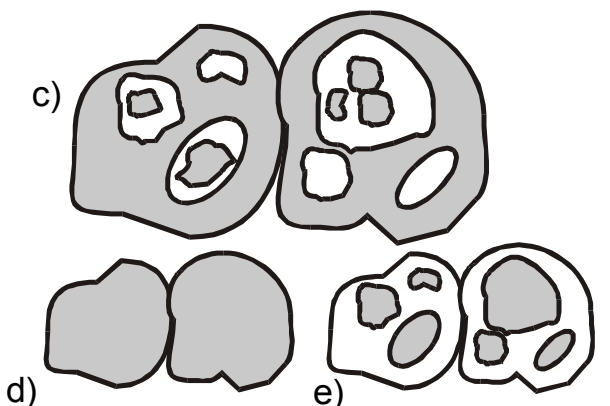

d)

e)

Abb. 21: Modell der topologischen Beziehungen nach WORBOYs \& BOFAKOS (1993): (a) Grundfläche (b) Skelett der Menge $\mathrm{A}=\{\mathrm{a}, \ldots, \mathrm{f}\}$, Beispiele des Einschlusses der generischen Fläche: (c) Geoobjekt,

(d) Grundfläche der 1. Ebene, (e) Grundfläche der zweiten Ebene.

Für eine endliche Menge von Atomen wird als Grundfläche (base area) eingeführt, wenn gilt:

(1) Für jedes Paar von unterschiedlichen Atomen in $A$ gilt, dass ihre Schnittmenge entweder leer oder ein Singleton (einelementige Menge) ist. A darf also keine zwei Atome haben, deren Vereinigung auch ein Atom bilden würde.

(2) $\Sigma A$ ist azyklisch.

Eine wichtige Eigenschaft der Grundfläche ist, dass sie eine eindeutige topologische Beschreibung erlaubt. Zwei Flächen mit gleichem Einschluss müssen auch die gleiche Repräsentation in Atomen und Skelett haben.

Sei $\mathrm{T}=<\mathrm{V}_{\mathrm{T}}, \mathrm{E}_{\mathrm{T}}>$ ein Baum mit einer Menge der Knoten $\mathrm{V}_{\mathrm{T}}$ und einer Menge der Kanten $\mathrm{E}_{\mathrm{T}}$. Für jeden Knoten x definiert s(x) die Menge seiner unmittelbaren Nachfolger.

Eine generische Fläche (generic area) G ist ein Baum, dessen Knoten, die nicht Wurzelknoten sind, mit den Namen von Atomen bezeichnet sind:

- für jeden Knoten $\mathrm{v} \in \mathrm{V}_{\mathrm{G}},\{\mathrm{W} \mid \mathrm{w} \in \mathrm{s}(\mathrm{v})\}$ ist Grundfläche

- für jeden Knoten $v \in V_{G}$, der nicht Wurzelknoten ist, und jede $\mathrm{w} \in \mathrm{s}(\mathrm{v})$ gilt: $\mathrm{w} \subset \mathrm{v}$ und $\mathrm{w} \cap \partial \mathrm{v}$ hat endliche Kardinalität.

Die Tiefe der generischen Fläche ist definiert als die Tiefe des Baumes, d.h. die Anzahl der Kanten auf dem gerichteten Pfad zwischen der Wurzel und den Ebenen. Die n-te Ebene der generischen Fläche bezeichnet die Menge der Atome in der Entfernung n von der Wurzel.

\subsubsection{Die Beziehungen in $\mathrm{Z}^{2}$}

Die Zellen einer Rasterstruktur unterscheiden sich von den Objekten in Vektorstrukturen dadurch, dass ihre Ränder räumlich ausgedehnt (2D) ist. EGENHOFER et al. (1993c) haben für Objekte, die aus Rasterzellen bestehen, 16 Beziehungen abgeleitet, die alle Beziehungen der 4-Intersection Matrix für Vektorobjekte enthalten. 

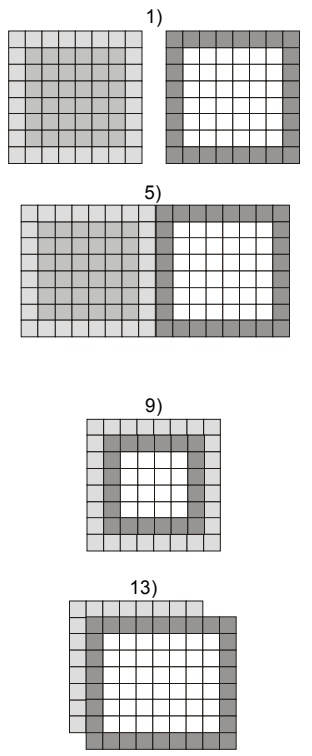

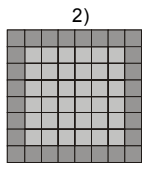

6)
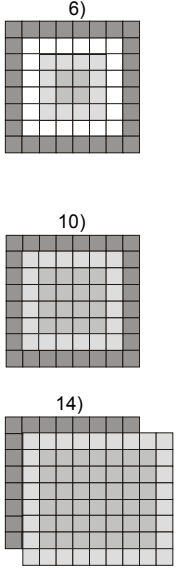
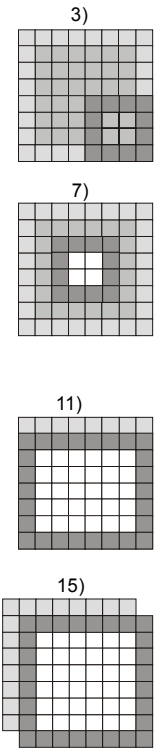
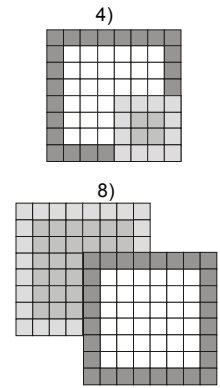

12)
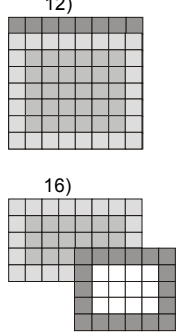

Abb. 22: Topologische Beziehungen zwischen den Objekten in Raster Tesselation.

Das 4-Nachbarn-Modell beschreibt die Beziehungen zwischen Objekten, deren Rand und Inneres nicht leer sind, die Ränder 4-verbunden (connected) sind und die keine Löcher besitzen. Jeder Punkt des Randes muss genau zwei 4-Nachbarn haben, um 4-verbunden zu sein. Ist der Rand 4verbunden, so gibt es keine zwei Punkte, einen im Äußeren und einen im Inneren, die 4- oder 8verbunden sind. Das Innere von solchen Objekten ist 4- verbunden und jeder seiner Punkte besitzt mindestens 3 8-Nachbarn. Das Modell geht von der Inhaltsinvariante aus (leere und nicht leere Schnittmengen).

Bei Anwendung von zwei zusätzlichen Bedingungen, die in $\mathbb{R}^{2}$ gelten, in $Z^{2}$ jedoch nicht, würden die 16 Beziehungen auf 8 mögliche zusammenschrumpfen:

- Wenn das Innere von A das Innere von B schneidet, dann muss es auch das Äußere von B schneiden und umgekehrt. Bei Anwendung dieser Bedingung würden die Beziehungen 9-15 (Abbildung 22) in $Z^{2}$ ihre Gültigkeit verlieren.

- Wenn beide Inneren disjunkt sind, dann kann der Rand von A nicht das Innere von B schneiden und umgekehrt. Bei Anwendung dieser Bedingung wäre die Konfiguration 16 (Abbildung 22) ungültig.

\subsubsection{Weitere topologische Invarianten}

Das 4- bzw. 9-Intersection Modell ist zu allgemein, um alle vorstellbaren topologischen Beziehungen zu unterscheiden. Das Modell berücksichtig nur eine topologische Invariante, den Inhalt (leeren/nicht-leeren) der Schnittmengen einzelner Objektteile (Inhaltsinvariante, content invariant). Weitere topologische Invarianten sind beispielsweise die Dimension der Schnittmenge, die Anzahl der Trennungen der Schnittmengen und die Sequenz der getrennten Schnittmengen unterschiedlicher Dimension entlang des Randes.

Eine allgemeine Erweiterung des 4-Intersections Modells, die 4-Intersection Invariante (EGENHOFER 1993a), kann beliebige Invarianten aufnehmen. Sei $S$ eine der vier Schnittmengen $\left(\partial \mathrm{A} \cap \partial \mathrm{B}, \mathrm{A}^{\circ} \cap \mathrm{B}^{\circ}, \partial \mathrm{A} \cap \mathrm{B}^{\circ}, \mathrm{A}^{\circ} \cap \partial \mathrm{B}\right)$. Eine 4-intersection Invariante für die topologische Relation

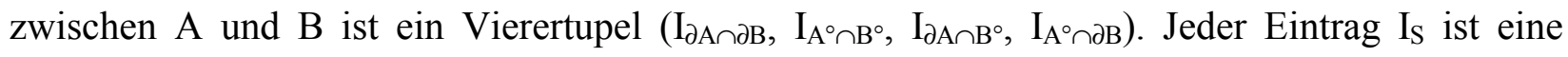


nichtleere Anzahl von Eigenschaften von S, wobei jede dieser Eigenschaften eine Invariante ist. Die Wahl der Invarianten für die 4-intersection Invariante kann in einer konkreten Situation detailliertere topologische Beziehungen unterscheiden.

\subsection{Komponenteninvarianten}

Die Komponenteninvariante baut auf den Komponenten und ihren Beziehungen auf. Eine Komponente von $\mathrm{Y}$ ist eine größte zusammenhängende Untermenge von Y. Sie ist topologisch invariant: Unter einer topologischen Transformation wird sie wieder in eine Komponente transformiert.

\section{Dimension der Komponente}

Die Dimension einer Komponente ist die Anzahl der Richtungen des Raumes, die zu ihrer eindeutigen Beschreibung nötig sind. Die Dimension einer Komponente ist -1 , falls sie leer ist, sonst höchstens gleich der Dimension des Raumes. In Abbildung 23a wird eine 0-dimensionale Komponente und in Abbildung 23b eine 1-dimensionale Komponente der boundary-boundary Komponente dargestellt.

\section{Boundary - Boundary Komponentensequenz}

Die Reihenfolge der Punkte an dem Rand eines Objektes bleibt unter topologischer Transformation ebenfalls erhalten. Die Punkte stellen in diesem Kontext die Komponenten der boundary-boundary Schnittmenge dar. Die Äquivalenz zweier Sequenzen $\mathrm{S}_{1}$ und $\mathrm{S}_{2}$ ist gegeben, wenn die Sequenz $S_{1}$ mit mindestens einer zyklischen Permutation (möglichen Sequenz) von $S_{2}$ identisch ist (Abbildung 23c-d).

Typ der boundary - boundary Komponente

Bei den Beziehungen mit nicht leeren boundary-boundary Schnittmengen können zwei Komponententypen unterschieden werden: (i) Komponente, deren zugehörige Kanten zwei unterschiedlichen Objektteilen angehören; (ii) Komponente, deren Kanten nur einem Objektteil angehören.

Sei C eine Komponente einer (nicht leeren) boundary - boundary Schnittmenge ( $\mathrm{A} \cap \partial \mathrm{B})$ in X. C wird als crossing Komponente bezeichnet, wenn für jede Umgebung $\mathrm{U}$ von $\mathrm{C}$ entweder $\left[\partial \mathrm{A} \cap \mathrm{U} \cap \mathrm{B}^{\circ} \neq \varnothing \vee \partial \mathrm{A} \cap \mathrm{U} \cap(\mathrm{X}-\mathrm{B}) \neq \varnothing\right]$ oder $\left[\partial \mathrm{B} \cap U \cap \mathrm{A}^{\circ} \neq \varnothing \vee \partial \mathrm{B} \cap \mathrm{U} \cap(\mathrm{X}-\mathrm{A}) \neq \varnothing\right]$ gilt. Eine Komponente, die nicht eine crossing Komponente ist, wird touching Komponente bezeichnet (Abbildung 23e-f).

Die crossing Komponente kann weiter verfeinert werden:

1. Aus der Kombination des Typs und der Dimension der Komponente ergeben sich für 1-D Komponenten zwei mögliche Zustände (Abbildung 23e):

- Komponente schneidet das Innere der Vereinigung beider Objekte = inner crossing

- Komponente liegt am Rand der Vereinigung beider Objekte = outer crossing

2. Aus der Kombination des Typs und der Orientierung der Ebene ergibt sich eine weitere Invariante: crossing direction. Dabei wird zwischen dem crossing in Konzept, wenn bei Traversieren des Randes von B eine Komponente am Übergang aus dem Äußeren in das Innere von A liegt, und dem crossing out, wenn die Komponente am Übergang aus dem Inneren in das Äußere von A liegt, unterschieden (Abbildung 23g). 


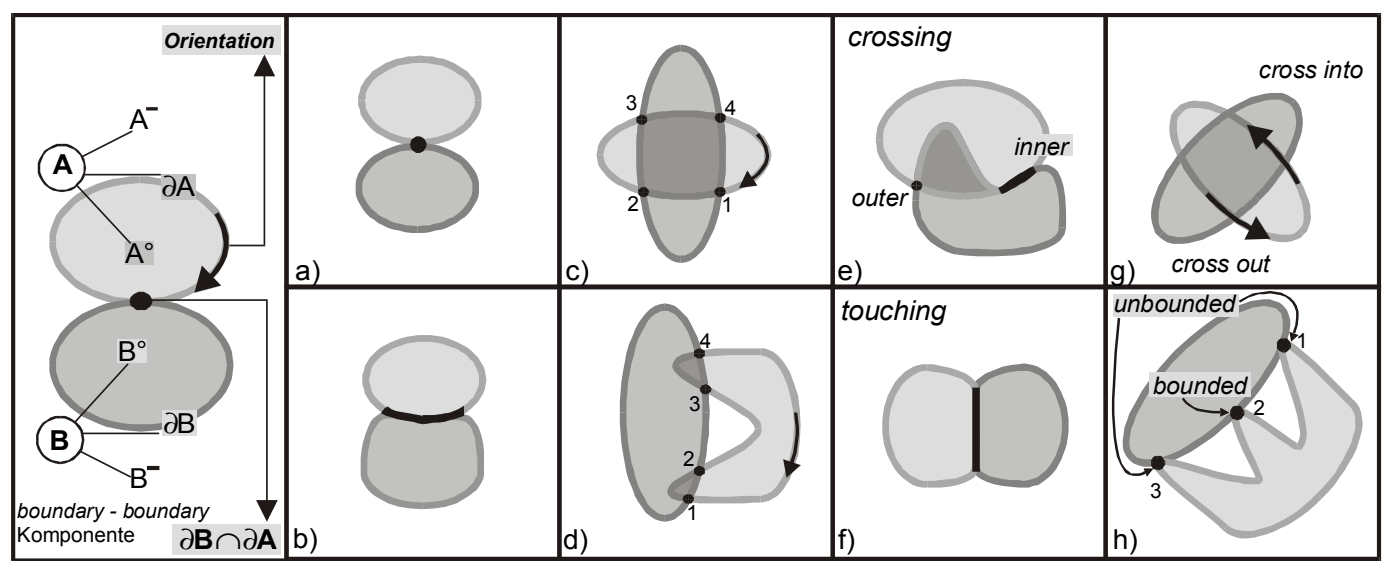

Abb. 23: Komponenteninvarianten als detailliertere topologische Modelle: a-b) Dimension der Komponente; c-d) Sequenz der Komponenten; e-f) Typ der boundary-boundary Komponente; g) crossing Richtung; h) complement Beziehung.

\section{Komplement-Beziehung}

Man kann auch die Beziehungen zwischen den Komponenten der boundary-boundary Schnittmenge und dem gemeinsamen exterior der Objekte untersuchen. Gemeinsames Äußeres ist das Komplement der Vereinigung beider Objekte. Es ist eine zusammenhängende offene Menge für die Situationen: disjount, equal, inside, contains, covers, coveredBy, und potentiell eine nicht-zusammenhängende Menge in den Situationen overlap und meet. Wenn das gemeinsame Äußere nicht zusammenhängend ist (mehrere Komponenten besitzt), ist gerade eine seiner Komponenten unbegrenzt (unbounded) und alle anderen begrenzt (bounded). Die boundary-boundary Komponenten, welche den Rand von einer unbegrenzten Komponente des gemeinsamen Äußeren schneiden, werden unbegrenzte Komponenten genannt, im anderen Fall heißen sie begrenzte Komponente (Abbildung 23h). Die topologische Invariante, die zwischen begrenzten und unbegrenzten Komponenten unterscheidet, wird nach EGENHOFER \& FRANZOSA (1994D) Komplementbeziehung genannt.

\subsection{Planar - disk classifying Invariante}

Das Modell der klassifizierenden Invarianten (EGENHOFER \& FRANZOSA 1994D) beschreibt die Kombinationen von Komponenteninvarianten. Durch Traversieren der $\partial \mathrm{B}$ erhält man für jede Komponente ein Tripel der Form:

\begin{tabular}{|c|c|c|c|}
\hline Komponentenzahl & (Komponentendimension & Komponententyp & Komplementbeziehung) \\
\hline & 0 oder 1 & touching/crossing & bounded/unbounded \\
\hline
\end{tabular}

Die topologischen Beziehungen zwischen den Objekten A und B und zwischen A' und B' sind äquivalent dann und nur dann, wenn sie die gleichen Eigenschaften der Inhaltinvariante haben, und es für die nicht-leere boundary-boundary Schnittmenge eine solche Orientierung und erste Komponente gibt, so dass die klassifizierende Invariante von A und B identisch mit der von A und $\mathrm{B}^{`}$ ist.

Nicht für jede topologische Konfiguration können alle Komponenteninvarianten angewendet werden. Ist beispielweise die boundary-boundary Schnittmenge leer, dann kann keine Erweiterung vorgenommen werden. Die Tabelle 4 fasst die Fälle zusammen, in welchen die Komponenteninvarianten die Inhaltsinvariante verfeinern können. 
Tab. 4: Die Beziehungen der Inhaltsinvariante, welche mit der Komponenteninvariante erweitert werden können (nach EGENHOFER \& FRANZOSA 1994D) .

\begin{tabular}{|l|l|l|l|l|l|l|}
\cline { 2 - 7 } \multicolumn{1}{l|}{} & Dimension & Sequenz & $\begin{array}{l}\text { Komplement } \\
\text { Beziehung }\end{array}$ & Typ & Begrenzung & $\begin{array}{l}\text { crossing } \\
\text { Richtung }\end{array}$ \\
\hline equal & & & & & & \\
\hline disjoint & & & & & & \\
\hline inside & & & & & & \\
\hline contains & & & & & & \\
\hline covers & $\mathrm{x}$ & $\mathrm{x}$ & & & & \\
\hline coveredBy & $\mathrm{x}$ & $\mathrm{x}$ & $\mathrm{x}$ & & & \\
\hline meet & $\mathrm{x}$ & $\mathrm{x}$ & $\mathrm{x}$ & & & \\
\hline overlap & $\mathrm{x}$ & $\mathrm{x}$ & $\mathrm{x}$ & $\mathrm{x}$ & $\mathrm{x}^{1}$ & $\mathrm{x}^{2}$ \\
\hline $\begin{array}{l}1 \\
\text { Nur für crossing boundary-boundary Komponente } \\
{ }^{2} \text { Nur für 1-D boundary -boundary Konponente }\end{array}$ \\
\hline
\end{tabular}

\subsubsection{Beziehungen für Polygone mit Löchern}

Ein Polygon mit Löchern teilt das Äußere in zwei Mengen: ein outer exterior $A_{0}^{-}$und mindestens ein inner exterior $A_{1}^{-} \ldots A_{n}^{-}$. Ihre Vereinigung bildet das gesamte Äußere.

$A^{-}=\bigcup_{i=0}^{n} A_{i}^{-}$

Je nachdem, welche Beziehungen zwischen einem Objekt und seinen Löchern und zwischen den Löchern untereinander erlaubt sind, können zwei Ansätze unterschieden werden (EGENHOFER et al. 1994c):

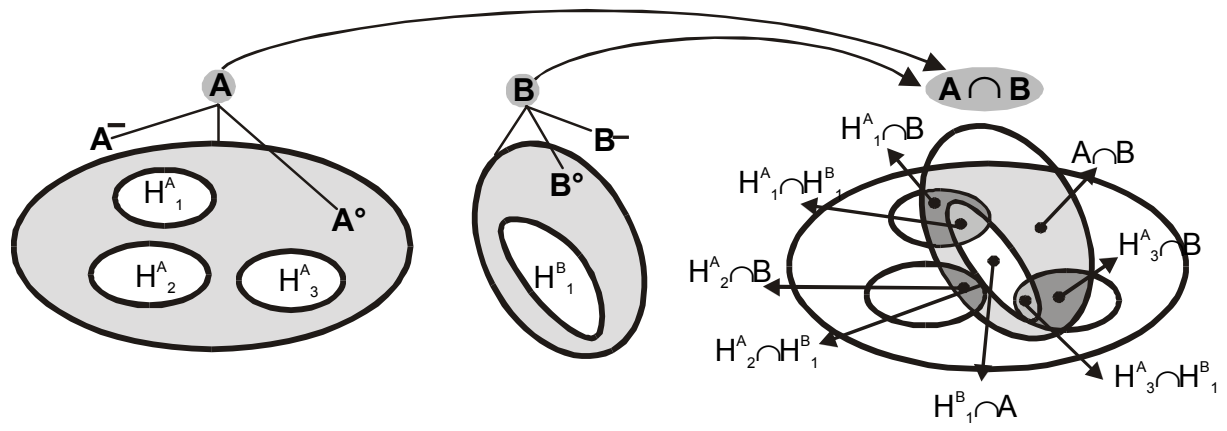

Abb. 24: Ableitung topologischer Beziehungen zwischen Geoobjekten mit Löchern.

- Ansatz 1 lässt nur solche Löcher zu, die vollständig im Polygon enthalten (in) und voneinander disjunkt sind.

- Ansatz 2 lässt auch solche Löcher zu, die am Polygonrand liegen (coveredBy) und sich berühren (meet).

Nach Ansatz 1 kann ein Objekt $A$ mit Löchern als geschlossene Hülle des Inneren von $A$ definiert werden. Ein Loch (hole) von $A\left(H^{A}\right)$ wird als geschlossene Hülle vom inneren Äußeren (inner exterior) beschrieben: $H_{i}^{A}=\bar{A}_{i}^{-}$. Die Vereinigung des Objektes A mit seinen Löchern wird als generalized region $A^{*}$ bezeichnet. Dieses Konzept erlaubt es, ein Polygon mit Löchern als eine Menge simpler Polygone ohne Löcher aufzufassen. Durch die Zerlegung eines Objektes mit 
Löchern in einfache Objekte $\left(A^{*}, H_{1}^{A}, \ldots H_{n}^{A}\right)$ ohne Löcher können ihre topologischen Beziehungen als Menge der 4-Intersection modelliert werden (Abbildung 24).

Nach Ansatz 2 kann das Objekt mit Löchern als eine nicht-leere Untermenge des Raumes mit zusammenhängendem Inneren definiert werden, so dass die Union des Objektes und aller seiner Löcher gleich der geschlossenen Hülle der Vereinigung des Objektinneren und aller seiner inneren Äußeren (inner exterior) ist. In diesem Ansatz ist die Menge der möglichen Beziehungen zwischen dem Objekt A und seinen Löchern und zwischen den Löcher untereinander größer als in Ansatz 1: $A^{*} r_{\text {(containsvcoversvequal) }} H_{i}^{A} ; H_{j}^{A} r_{(\text {disjointvmeet })} H_{i}^{A}$.

\subsubsection{Quantifikation der topologischen Beziehungen}

Das $\mathrm{Ma} \beta$ der topologischen Entfernung (topological distance) kann zur Beurteilung der Ähnlichkeit der topologischen Beziehungen herangezogen werden. Es beschreibt die Anzahl der unterschiedlichen Werte in zwei entsprechenden 9-intersections Matrizen. "The topology distance between two 9-intersections is the smallest number of 'bits' that must be flipped to convert one 9intersection into the other" (EGENHOFER \& AL-TAHA 1992b).

Die topologische Entfernung kann als Summe der absoluten Differenzen zwischen entsprechenden Elementen der 9-Intersection Matrix bestimmt werden.

$$
\tau_{r A, r B}=\sum_{i=\partial}^{-} \sum_{j=\partial}^{-}\left|M_{A}[i, j]-M_{B}[i, j]\right|
$$

Der Wert der topologischen Entfernung einer Relation zu sich selbst beträgt 0 , zwischen zwei beliebigen Relationen liegt er zwischen 0 und 8 (Tabelle 5). Das Maß $\tau$ erfüllt die Voraussetzungen einer Metrik: es ist symmetrisch $\left(\tau_{\mathrm{rA}, \mathrm{rB}}=\tau_{\mathrm{rB}, \mathrm{rA}}\right)$, größer oder gleich 0 und die Summe der topologischen Entfernungen zwischen den Relationen $r_{A}, r_{B}$ und $r_{B}, r_{C}$ ist größer oder gleich der topologischen Entfernung der Relationen $\mathrm{r}_{\mathrm{A}}, \mathrm{r}_{\mathrm{C}}$ (Dreiecksungleichung):

$$
\tau_{\mathrm{rA}, \mathrm{rB}}+\tau_{\mathrm{rB}, \mathrm{rC}} \geq \tau_{\mathrm{rA}, \mathrm{rC}} .
$$

Tab. 5: Topologische Entfernungen der 8 Polygon-Polygon Relationen (nach EGENHOFER \& AL-TAHA 1992B).

\begin{tabular}{|c|c|c|c|c|c|c|c|c|}
\cline { 2 - 10 } \multicolumn{1}{c|}{} & disjoint & meet & equal & inside & coveredBy & contains & cover & overlap \\
\hline disjoint & 0 & $\mathbf{1}$ & 6 & 4 & 5 & 4 & 5 & 4 \\
\hline meet & $\mathbf{1}$ & 0 & 5 & 5 & 4 & 5 & 4 & $\mathbf{3}$ \\
\hline equal & 6 & 5 & 0 & 4 & 3 & 4 & 3 & 6 \\
\hline inside & 4 & 5 & 4 & 0 & $\mathbf{1}$ & 6 & 7 & 4 \\
\hline coveredBy & 5 & 4 & $\mathbf{3}$ & $\mathbf{1}$ & 0 & 7 & 6 & $\mathbf{3}$ \\
\hline contains & 4 & 5 & 4 & 6 & 7 & 0 & $\mathbf{1}$ & 4 \\
\hline cover & 5 & 4 & $\mathbf{3}$ & 7 & 6 & $\mathbf{1}$ & 0 & 3 \\
\hline overlap & 4 & 3 & 6 & 4 & 3 & 4 & 3 & 0 \\
\hline
\end{tabular}

Die kleinste topologische Entfernung ist eindeutig für disjoint : meet, inside : coveredBy und contains : cover. Sie ist mehrdeutig für die Paare mit equal und overlap $(\tau=3)$. Aus Tabelle 5 wird klar, dass die kleinste topologische Entfernung nicht unbedingt symmetrisch sein muss.

Die Relationen können bezüglich der Zeitdauer ihrer Existenz in dominante, stark dominante und dominierte Relationen gegliedert werden (WINTER 1996). Eine Relation ist dominant gegenüber 
ihren Nachbarn, wenn sie während einer stetigen Deformation nur zu einem Zeitpunkt existieren kann. Als Beispiel kann die Relation meet dienen, die zu ihren Nachbarn in dominanter Stellung steht. Die Menge der dominanten Relationen besteht aus \{meet, covers, coveredBy, equal\}. Die dominierten Relationen sind \{disjoint, intersect, contains, inside\} (Abbildung 17).

Die Beschreibung der topologischen Beziehungen kann mit metrischen Konzepten zweifach erweitert werden (EGENHOFER \& SHARIFF 1998):

- Splitting bezeichnet die Quantifikation der nicht-leeren Schnittmengen zweier Objekte (Abbildung 25a-f).

- Closeness beschreibt die Quantifikation der Beziehungen zweier Objektteile zueinander, deren Schnittmenge leer ist (Abbildung 25g-h).

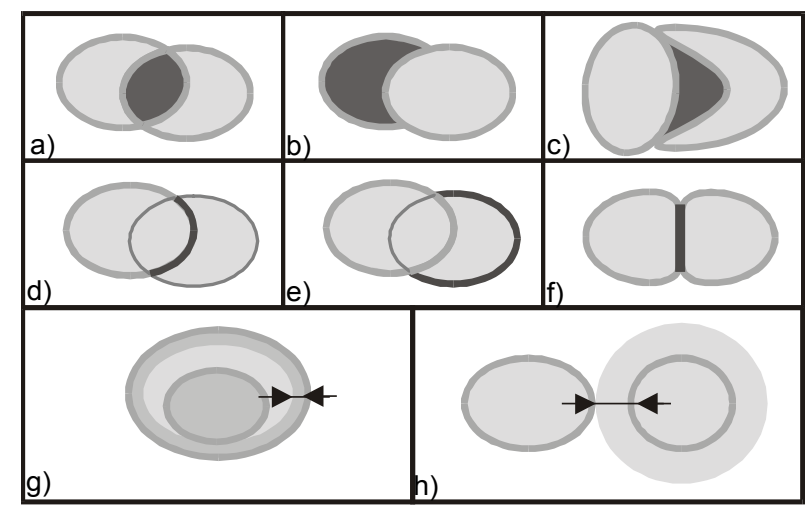

Abb. 25: Metrische Konzepte zur Beschreibung von topologischen Beziehungen (in Anlehnung an EGENHOFER \& SHARIFF 1998): a)inner area splitting; b) outer area splitting; exterior splitting; d) inner traversal splitting; e) outer traversal splitting; f) alongness splitting; g) inner closeness; h) outer closeness.

Splitting bestimmt, wie das Innere, der Rand und das Äußere von einem 2D Geoobjekt durch Inneres und Rand eines 1D Geoobjektes zerlegt wird und umgekehrt. Die metrischen Konzepte, die zur Beschreibung einer nicht-leeren Schnittmenge angewendet werden können, umfassen die Länge bei linienartigen (das Innere einer Linie L, der Rand eines Polygons R) und die Fläche bei flächenartigen Objekten (das Innere eines Polygons R).

\begin{tabular}{l|ccc} 
& $R^{\circ}$ & $\partial R$ & $R^{-}$ \\
\hline$L^{\circ}$ & Länge & Länge & Länge \\
$\partial L$ & - & - & - \\
$L^{-}$ & Fläche & Länge & Fläche
\end{tabular}

Closeness beschreibt die Disjunktheit anhand der Entfernung von Punkten und Linien näher.

\begin{tabular}{c|ccc} 
& $R^{\circ}$ & $\partial R$ & $R^{-}$ \\
\hline$L^{\circ}$ & - & Entfernung & - \\
$\partial L$ & - & Entfernung & - \\
$L^{-}$ & - & - & -
\end{tabular}

\subsubsection{Distanzbeziehungen}

Die Entfernung bezeichnet eine Beziehung zwischen dem Primär- (PO) und dem Referenzobjekt (RO) in einem Bezugssystem. Sie gibt die relativen Positionen oder die Größen-Eigenschaften an (Hernández 1998, HernÁndez et al. 1995, HernÁndez 1994, Clementini et al. 1995b). Die 
Bestimmung der Entfernung setzt die Existenz einer Metrik $d$ voraus. Ein metrischer Raum muss die Bedingungen der Reflexivität, der Symmetrie und der Dreiecksungleichung erfüllen. Neben der euklidischen Entfernung (Minkowski $\mathrm{L}_{2}$ Metrik) können weitere Metriken angewendet werden (City -block oder Manhattan distance $\mathrm{L}_{1}$ Metrik, die Netzwerk-Entfernung usw.).

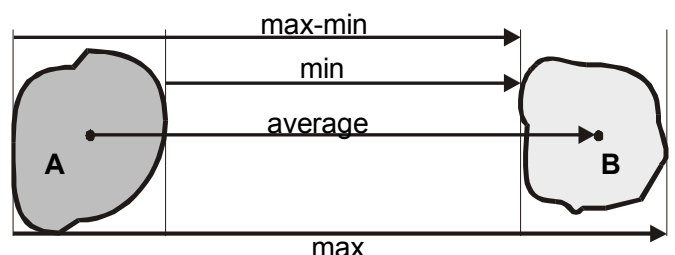

a)

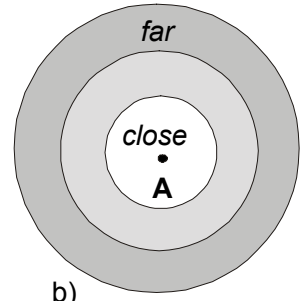

b)

Abb. 26: Unterschiedliche Typen der Distanzbeziehungen: (a) quantitative Entfernung, (b) qualitative Entfernungsbeziehungen.

Menschliche Entfernungskonzepte hängen von vielen Faktoren ab (HERNÁNDEZ et al. 1995): Metrischer Entfernung, Zeitaufwand, Kosten, Wahrnehmung, relativer Größe und Form, Position anderer Objekte, Bezugsrahmen. "What is means for $A$ to be near $B$ depends not only on their absolute positions (and the metric distance between them), but also on their relative sizes and shapes, the position of other objects, the frame of reference, and 'what it takes to go from A to $B$ '" (HERNÁNDEZ et al. 1995). Die Messgröße der Entfernung muss diese Konzepte in einer konkreten Situation berücksichtigen. Sie kann eine räumliche (metrische Entfernung), zeitliche (Reisezeit), ökonomische (Reisekosten) oder kognitive (räumliche Wahrnehmung) Größe sein. Die Entfernungskonzepte können isotrop (der Bewegungsaufwand ist gleich in allen Richtungen von jedem Punkt aus) oder anisotrop sein: der Bewegungsaufwand hängt von der Bewegungsrichtung und dem Ausgangspunkt ab. Anisotropische Räume können durch eine Transformation oft in isotropische überführt werden.

Bei 0-dimensionalen Geoobjekten (Punkten) ist die Entfernung eindeutig definiert. Bei räumlich ausgedehnten Objekten ist die Bestimmung ihrer Entfernung komplizierter (Abbildung 26). Eine triviale Lösung für diesen Fall ist eine punktförmige Annährung (Approximation) beider ausgedehnter Objekte. Weitere Ansätze betrachten die kleinste, größte oder durchschnittliche Entfernung (OKABE \& MILLER 1996). Die durchschnittliche Entfernung (average distance) wird oft durch die Entfernung der Schwerpunkte beider Objekte beschrieben.

$$
d_{\text {avg }}(A, B)=\frac{1}{S_{A} S_{B}} \int_{A} \int_{B} d\left(x_{a}, x_{b}\right) d x_{a} d x_{b}
$$

$\mathrm{S}_{\mathrm{A}}, \mathrm{S}_{\mathrm{B}}-$ Flächen der Geoobjekte A und B

$x_{a}, x_{b}$ - Position in den Geoobjekten

$d\left(x_{a}, x_{b}\right)$ - Entfernung der Positionen $\mathrm{x}_{\mathrm{a}}$ und $\mathrm{x}_{\mathrm{b}}$

Die minimale und die maximale Entfernung stellen die Extreme der Entfernung dar. Bei regulär geschlossenen Punktmengen entsprechen die minimale und die maximale Entfernung der Entfernung zwischen zwei Grenzpunkten (Abbildung 26).

$$
\begin{aligned}
& d_{\text {min }}(A, B)=x_{a} x_{b}\left\{d\left(x_{a}, x_{b}\right) \mid x_{a} \in A, x_{b} \in B\right\} \\
& d_{\text {max }}(A, B)=x_{a} x_{b}\left\{d\left(x_{a}, x_{b}\right) \mid x_{a} \in A, x_{b} \in B\right\}
\end{aligned}
$$


Die Hausdorff-Entfernung ist ein Max-min Konzept der Entfernung. Sie stellt das Maximum der kürzesten Entfernung zwischen einem beliebigen Punkt eines Objektes und dem nächsten Punkt des Zweiten dar (Abbildung 26).

In der order-neighbour Analyse wird die Entfernung- mit der Ordnungsrelation kombiniert. Die sog. R-statistischen Methoden (APLIN 1983) sind auf der Entfernung basierte Methoden der Untersuchung von Point-pattern. Dabei werden für eine Punktmenge die ersten bis k-ten Nachbarpunkte unbeachtet der Richtung gesucht. Der erste Nachbar ersetzt in manchen Fällen die topologische Beziehung Adjazenz. Innerhalb der vorher definierten Nachbarschaft sorgt die Distanz meistens auch für die Zuteilung der entsprechenden Gewichte der einzelnen Nachbarn.

Die Kombination der metrischen und topologischen Konzepte wird von GaLTON (1997) zur Unterscheidung mehrerer, Trennungsgrößen' angewendet: boundary separation, size separation, interior separation.

- Die boundary separation ist als die kleinste Entfernung $d$ zweier Polygone $\mathrm{R}_{1}, \mathrm{R}_{2}$ definiert, innerhalb welcher jeder Punkt des Randes $\partial \mathrm{R}_{1}$ von einem Punkt des Randes $\partial \mathrm{R}_{2}$ entfernt ist.

- Size separation von zwei Polygonen ist die Fläche der symmetrischen Differenz: $\mathrm{R} 1 \Delta \mathrm{R} 2=\left(\mathrm{R}_{1} \backslash \mathrm{R}_{2}\right) \cap\left(\mathrm{R}_{2} \backslash \mathrm{R}_{1}\right)$.

- Interior separation ist die kleinste Entfernung $\mathrm{d}$, innerhalb welcher jeder Punkt eines Polygones von einem Punkt des zweiten Polygons entfernt ist. Die Polygone werden in diesem Fall als offen angenommen.

Die qualitativen Entfernungsbeziehungen von CLEMENTINI et al. (1995b) gehen von einer (endlichen) Menge von Entfernungssymbolen aus. Jedem Primärobjekt $(\mathrm{PO} \in \mathrm{O})$ wird ein Entfernungssymbol zugeordnet, das seine qualitative Entfernung vom Referenzobjekt $(\mathrm{RO} \in \mathrm{O})$ angibt. Die erste Granularitätsstufe ist close und far. Um die Entfernungssymbole miteinander vergleichen $\mathrm{zu}$ können, benötigt man eine Abbildung in 1-dimensionalen geometrischen Intervallen. Außerdem wird eine algebraische Struktur über Intervalle benötigt.

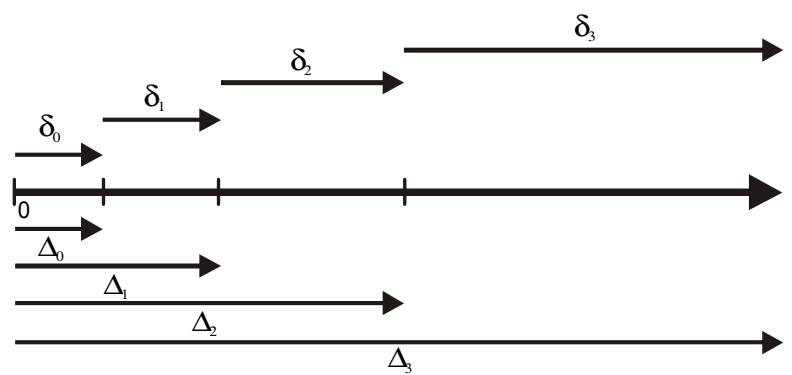

Abb. 27: Qualitative Entfernungsbeziehungen, welche die Beziehungen der Homogenität und range restriction erfüllen (In Anlehnung an CLEMENTINI et al. 1995b).

Sei $\mathrm{Q}=\left\{\mathrm{q}_{0}, \mathrm{q}_{1}, \mathrm{q}_{2}, \ldots, \mathrm{q}_{\mathrm{n}}\right\}$ die (endliche) Menge der Entfernungssymbole, wobei $\mathrm{q}_{0}$ die kleinste Entfernung vom Referenzobjekt bezeichnet. Die Funktion d: $\mathrm{O} \times \mathrm{O} \rightarrow \mathrm{Q}$ ordnet jedem Primärobjekt $(\mathrm{PO} \in \mathrm{O}$ ) ein Entfernungssymbol $\mathrm{zu}$, das seine qualitative Entfernung vom Referenzobjekt $(\mathrm{RO} \in \mathrm{O})$ angibt.

Wegen der linearen Ordnung der Menge Q können die Nachbarschaftssymbole bestimmt werden:

Funktion successor: $\quad \operatorname{succ}\left(\mathrm{q}_{\mathrm{i}}\right)=\mathrm{q}_{\mathrm{i}+1}($ für $\forall \mathrm{i}<\mathrm{n})$ 


$$
\begin{aligned}
& \operatorname{succ}\left(\mathrm{q}_{\mathrm{n}}\right)=\mathrm{q}_{\mathrm{n}} \\
\text { Funktion predecessor: } & \operatorname{pred}\left(\mathrm{q}_{\mathrm{i}}\right)=\mathrm{q}_{\mathrm{i}-1}(\text { für } \forall \mathrm{i}>0) \\
& \operatorname{pred}\left(\mathrm{q}_{0}\right)=\mathrm{q}_{0}
\end{aligned}
$$

Ein Entfernungssystem (distance system) wird in HERNÁNDEZ et al. (1995) definiert als $\mathrm{D}=(\mathrm{Q}, \mathrm{A}, \mathrm{I}) . Q$ ist eine (lineare) Ordnung der Entfernungsbeziehungen. $A$ ist eine Akzeptanzfunktion A: Q x O $\rightarrow$ I: Für ein Referenzobjekt RO gibt A( $\left.\mathrm{q}_{\mathrm{i}}, \mathrm{RO}\right)$ die Akzeptanzfläche $\left(\delta_{\mathrm{i}} \in \mathrm{I}\right)$ der Beziehung $\mathrm{q}_{\mathrm{i}}$ wieder. $I$ ist eine algebraische Struktur der Intervallmenge.

An das Modell der qualitativen Beziehungen können bestimmte Anforderungen gestellt werden (CLEMENTINI et al. 1995b) (Abbildung 27):

- Homogenität sorgt für ein sich wiederholendes Muster der Strukturrelationen.

- Monotonicity beschreibt einen Fall, in welchem jedes Intervall größer oder gleich dem Vorherigen ist. $\delta_{0} \leq \delta_{1} \leq \delta_{2} \ldots \leq \delta_{n}$

- Range restriction gibt an, dass jedes Intervall größer als die gesamte Spannweite von Anfang bis zu dem Vorgänger des gegebenen Intervalls ist.

- $\delta_{i} \geq \Delta_{i-1}, \forall i>0$

- Order of magnitude bedeutet, dass für eine gegebene Differenz zwischen der Ordnungszahl von zwei Entfernungsrelationen $\mathrm{q}_{i}$ und $\mathrm{q}_{\mathrm{j}}$ angenommen wird, dass $\mathrm{q}_{\mathrm{j}}$ viel größer als $q_{i}$ ist.

Die Komposition von zwei Entfernungsbeziehungen hängt von der Richtungsbeziehung zwischen den Objekten ab und umgekehrt:

Das Ergebnis einer Komposition der Richtungsbeziehungen $\theta(A, B)$ und $\theta(B, C)$ ist direkt von der Entfernung d(A,C) abhängig.

Das Ergebnis einer Komposition der Entfernungsbeziehungen d(A,B) und d(B,C) hängt von der Spannweite beider Ric htungsbeziehungen ab.

\subsubsection{Richtung}

Die Richtungsbeziehungen beschreiben die Ordnung des Raumes (THEODORIDIS et al. 1998). Sie geben an, wo die Objekte relativ zu einander liegen (CLEMENTINI et al. 1995b). Im Gegensatz zu den topologischen Beziehungen haben die Richtungsbeziehungen erst in den letzten Jahren an Bedeutung gewonnen. Bis jetzt gibt es keine universal anerkannte Definition der Richtungsbeziehungen (PAPADIAS et al. 1996).

Die Richtungsbeziehungen sind primär fuzzy Größen: "Unlike other spatial relationships such as distance, adjacency or containment however, direction is a fuzzy concept and is thus often dependent on human interpretation" (PEQUET \& CI-XIANG 1987). Die Richtungsrelationen sind invariant unter affiner Transformation.

Eine Richtungsrelation entsteht durch die Projektion antromorph bestimmter Achsen auf das Bezugsobjekt (HERNÁNDEZ 1998). Bei der Bestimmung der Beziehung benötigt man ein primäres Objekt, dessen relative Position zu bestimmen ist, ein Referenzobjekt, in bezug auf welches die Richtung bestimmt wird, und einen Bezugsrahmen (frame of reference). Der Bezugsrahmen entscheidet über die Vorderseite des Referenzobjektes, und daher über den Namen der Beziehung 
(Clementini et al. 1995b). Man unterscheidet drei Typen des Bezugsrahmens: Im intrinsischen erfolgt die Orientierung des Referenzobjektes anhand einer seiner inhärenten Eigenschaften (z.B. Bewegungsrichtung). Der extrinsische Bezugsrahmen verwendet einen externen Faktor zur Orientierung des Referenzobjektes. Im deiktischen Modell ergibt sich die Orientierung des Referenzobjektes aus dem Standpunkt, aus welchen das Referenzobjekt angesehen wird.

Die Richtungsbeziehung ist binär, sie verbindet genau zwei Objekte (PEUQUeT \& CI-XIANG 1987). Jede Richtungsrelation impliziert eine inverse Beziehung. Die Richtungsbeziehungen haben eine uniforme kreisförmige Nachbarschaftsstruktur (CLEMENTINI et al. 1995b). Sei $\left\{\alpha_{0}, \alpha_{1}\right.$, $\left.\ldots, \alpha_{n}\right\}$ die Menge der Richtungsbeziehungen. Die Richtungsbeziehung von B in bezug auf A (A ist das Referenz- und $B$ das Primärobjekt) ist das Ergebnis der Funktion $\theta_{A B}=\theta(A, B)$, die einen beliebigen Wert $\alpha_{i}$ annehmen kann. Die Nachbarschaftsstruktur erlaubt die Definition von Vorgänger und Nachfolger:

$$
\begin{aligned}
& \text { Funktion successor: } \operatorname{succ}\left(\alpha_{0}\right)=\alpha_{1}, \operatorname{succ}\left(\alpha_{1}\right)=\alpha_{2} \\
& \operatorname{succ}\left(\alpha_{n}\right)=\alpha_{0} \\
& \text { Funktion predecessor: } \operatorname{pred}\left(\alpha_{0}\right)=\alpha_{\mathrm{n}}, \operatorname{pred}\left(\alpha_{1}\right)=\alpha_{0} \\
& \operatorname{pred}\left(\alpha_{\mathrm{n}}\right)=\alpha_{\mathrm{n}-1}
\end{aligned}
$$

Wegen der Kreisförmigkeit der Nachbarschaftsstruktur ist der ( $\mathrm{n}+1 / 2)$-te Vorgänger identisch mit $\operatorname{dem}(\mathrm{n}+1 / 2)$-ten Nachfolger.

Die Spannweite zwischen zwei Richtungen wird als die kleinste Anzahl der Schritte definiert, die nötig sind, um aus einer die andere durch die Nachbarschaftsstruktur zu erreichen. Die Spannweite ist gleichzeitig die Menge der möglichen Ergebnisse der Komposition zweier Beziehungen. Zwei Beziehungen sind orthogonal, wenn ihre Spannweite $(n+1) / 4$ ist.

$$
\text { Funktion orthogonal: } \operatorname{orth}(\alpha \mathrm{i})=\left\{\operatorname{succ}((\mathrm{n}+1) / 4)\left(\alpha_{\mathrm{i}}\right) ; \operatorname{pred}((\mathrm{n}+1) / 4)\left(\alpha_{\mathrm{i}}\right)\right\}
$$

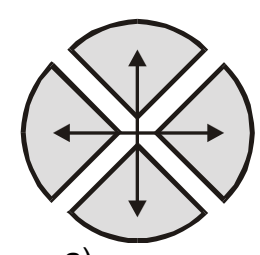

a)

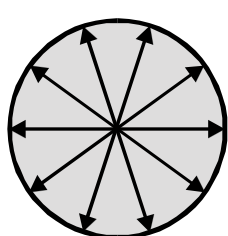

b)

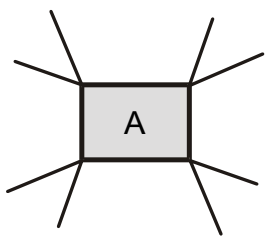

c)

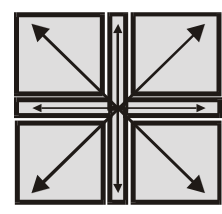

d)

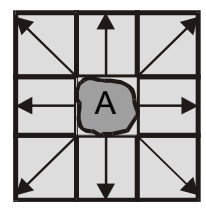

e)

Abb. 28: Ansätze der Richtungsbeziehungen: (a-c) Kegelformansatz, (d-e) projektionsbasierter Ansatz.

Die Fläche, für welche eine Beziehung zutrifft, wird als Akzeptanzfläche (acceptance area) bezeichnet. Es werden zwei unterschiedliche Ansätze der Richtungsbeziehungen unterschieden: die projektionsbasierten Relationen und die Relationen der konischen Bereiche (Abbildung 28). 


\subsubsection{Relationen der konischen Bereiche (Kegelform-Ansatz)}

Bei diesem Ansatz wird die Richtung als der Winkel definiert, den die Linie, die das Primär- und das Referenzobjekt verbindet, mit einer im Raum fixierten Richtung ausmacht (vgl. FRANK 1996a). Die Position des Primärobjektes wird mit dem dreieckigen Akzeptanzraum verglichen, der vom Mittelpunkt des Referenzobjektes ausgeht. Die charakteristische Eigenschaft dieses Ansatzes ist die Vergrößerung des Akzeptanzraumes mit der Entfernung von Referenzobjekt (Abbildung 28a-c). Nach dieser Eigenschaft wird er auch Dreiecksansatz genannt.

Bei kardinalen Richtungen (Himmelsrichtungen) im large scale space sind zwei Mengen der Richtungsbeziehungen von Bedeutung (FRANK 1996a):

$\mathrm{V}_{4}=\{\mathrm{N}, \mathrm{W}, \mathrm{S}, \mathrm{E}\}$ oder $\mathrm{V}_{9}=\{\mathrm{N}, \mathrm{NE}, \mathrm{E}, \mathrm{SE}, \mathrm{S}, \mathrm{SW}, \mathrm{W}, \mathrm{NW}, 0\}$

Bei den Objekten mit Ausdehnung stellt sich das Problem der Bestimmung der Richtungsrelation, da in diesem Fall auch relative Größe, Entfernung und Form die Richtung beeinflussen (Peuquet \& Ci-XIANG 1987) (Abbildung 29a-b). Die auf diesem Ansatz basierten Beziehungen weisen zusätzliche Eigenschaften auf:

- Die Akzeptanzfläche einer Beziehung wächst mit der Entfernung, d.h. eine Richtungsbeziehung wird um so eher akzeptiert, je weiter die zwei Objekte entfernt sind.

- Eine Richtungsbeziehung wird um so eher akzeptiert, je größer das Referenzobjekt im Vergleich zum Primärobjekt ist.

- Bei der Wahl des Referenzobjektes ist dasjenige auszuwählen, das visuell dominant ist.

Das Modell von Peuquet (Peuquet \& Ci-XIANG 1987) beruht auf dem Kegelformansatz. Der Ansatz wird mit Regeln erweitert, die das Beeinflussen der Beziehungen durch die Größe und Entfernung der beiden Objekte in Betracht ziehen. Bei der Bestimmung der Beziehung unterscheidet man drei Fälle:

A. Das Primärobjekt liegt komplett außerhalb der vordefinierten Entfernung vom Referenzobjekt. In diesem Fall ist die Bestimmung der Richtung auf zwei Punkte reduziert. Beide Objekte werden dabei durch einen Punkt approximiert. Bei der Bestimmung der Richtungsrelation wird diejenige (dreieckförmige) Richtung gesucht, in die die Approximation des Primärobjektes fällt (Abbildung $29 a-b)$.

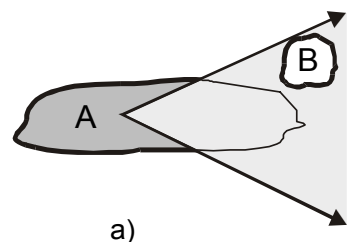

a)

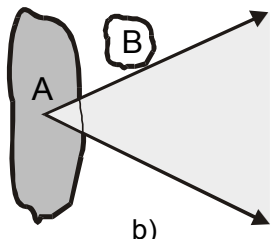

b)

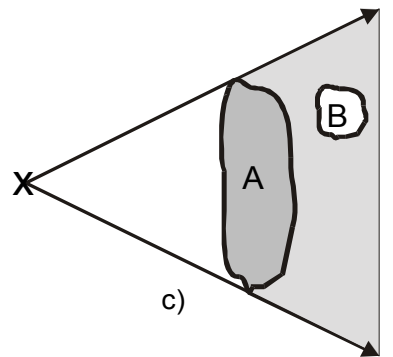

Abb. 29: Schwierigkeiten der Ableitung der Richtungsbeziehungen für räumlich ausgedehnte Objekte: a) Richtungsbeziehung trifft zu, obwohl es nicht der Fall ist; b) Beziehungen trifft nicht zu, obwohl es der Fall ist; c) Ableitung der Richtungsbeziehung durch den Scheitelpunkt.

B. Die Objekte stehen relativ nah zueinander. In diesem Fall werden die Objekte mit dem minimal umgebenden Rechteck approximiert. Die maximale Dimension der Approximation (d.h. 
die größte Seitenlänge) des Referenzobjektes hat einen großen Einfluss auf die Bestimmung der Relation, während die kleinere Dimension die Bestimmung kaum beeinflusst. Diese Tatsache kann bei der Bestimmung berücksichtigt werden, indem der Scheitelpunkt des Dreiecks (Zentroid des Referenzobjektes) in die Richtung oder Gegenrichtung der Beziehung verschoben wird bis die Ecken der Approximation an den Seiten des Dreiecks liegen (Abbildung 29c).

C: Die beiden Objekte sind miteinander verflochten, d.h. das Zentroid eines Objektes fällt in die Approximationsfläche des zweiten. Für diese Situation, in welcher selbst die visuelle Beurteilung schwierig ist, werden zusätzliche Bedingungen definiert. Man bildet eine zu den Koordinatenachsen parallel verlaufende Linie mit Zentrum im Zentroid des Objektes und mit der Richtung der vermuteten Richtungsbeziehung. An dem Inhalt der Schnittmengen der Linien mit dem Primärobjekt können Bedingungen definiert werden, unter welchen eine Richtungsbeziehung zustande kommt.

\subsubsection{Projektionsbasierte Relationen}

Die projektionsbasierten Richtungsbeziehungen werden durch zwei zu den Koordinatenachsen senkrecht verlaufende Projektionslinien definiert. Der Raum wird durch zwei senkrechte Linien (Achsenkreuz) mit dem Zentrum im Referenzobjekt geteilt. Jede Linie erzeugt zwei Halbebenen, insgesamt entstehen vier Quadranten. Die vier Quadranten mit vier Halbgeraden und Zentrum bilden mit 9 unterschiedlichen und den ganzen Raum deckenden Beziehungen die höchste Auflösung dieses Ansatzes. Nach ihrem Akzeptanzraum können die Beziehungen wie folgt unterteilt werden:

- $\quad$ D (Punkt) - das Zentrum bzw. der Kreuzpunkt der Achsengeraden $\{0\}$

- 1D (Linie) - die vier Halbgeraden, die durch Kreuzung der Achsengeraden entstehen

- 2D (Quadrant) - die vier semioffenen Quadranten, die die Achsengeraden im Raum abgrenzen

Die Relationen haben unterschiedliche Zeitdauer bei einer graduellen Deformation. In einigen Ansätzen werden jedoch die punkt- und linienartigen Richtungen ignoriert: $V_{4}=\{\mathrm{NE}, \mathrm{NW}, \mathrm{SE}$, $\mathrm{SW}$.

In dem Fall, dass das Primärobjekt nah zum Mittelpunkt des Achsenkreuzes liegt, muss es nicht klar sein, welchem Quadrant es zugeordnet werden soll. In diesem Fall ist es sinnvoll, den Raum in neun Quadranten einzuteilen. Diese Einteilung besteht aus einem neutralen Quadrant, vier Quadranten mit nur einer Richtung und vier Quadranten mit jeweils zwei Richtungen: $V_{9}=\{N E$, N, NW, E, W, 0, SE, S, SW .

Werden auch Richtungen mit einem Akzeptanzraum 1D berücksichtigt, ergeben sich insgesamt 17 unterschiedliche Richtungen. Der neutrale Quadrant kann als minimal umschließendes Rechteck des Referenzobjektes gebildet werden. Das Primärobjekt kann in seiner Form oder in seiner Approximation im Modell angewendet werden.

Das $2 D$ range queries Modell (PAPADIAS 1994, PAPADIAS et al. 1996) stützt sich auf den Projektionsansatz. Die Richtung für punktförmige Geoobjekte wird festgelegt, indem der Kreuzpunkt der Projektionslinien auf den Referenzpunkt gelegt wird. In dem Modell können Richtungsbeziehungen von unterschiedlichem Auflösungsgrad abgeleitet werden. Für jede Beziehung werden zwei Varianten (strong, weak) gebildet, die die Erfüllung der Bedingungen ausdrücken. 
Direction-relation Matrix DRM (GOYAL \& EGENHOFER 1997) basiert auf dem projektionsorientierten Ansatz mit Nullfläche um das Referenzobjekt (Abbildung 30). Es werden also 9 Richtungen unterschieden. Das Primärobjekt wird in seiner aktuellen Form betrachtet. Die 9 Schnittmengen des POs und des minimal umgebenden Rechtecks (MBR) des ROs (ähnlich wie bei 9-Intersectionmatrix) bilden eine Matrix, deren Elemente den Inhalt der Schnittmenge des POs und der Akzeptanzflächen der Relationen beschreiben. Das grobe DRM Modell unterscheidet nur zwischen leeren und nicht-leeren Schnittmengen $(\varnothing, \neg \varnothing)$.

$$
D R M=\left[\begin{array}{ccc}
n w & n & n e \\
w & \text { same } & e \\
s w & s & s e
\end{array}\right] \quad \operatorname{dir}_{R R}(A, B)=\left[\begin{array}{ccc}
N W_{A} \cap B & N_{A} \cap B & N E_{A} \cap B \\
W_{A} \cap B & 0_{A} \cap B & E_{A} \cap B \\
S W_{A} \cap B & S_{A} \cap B & S E_{A} \cap B
\end{array}\right]
$$

Das detaillierte DRM Modell verfeinert die Beschreibung der Schnittmenge mit ihrer relativen Größe zum Primärobjekt, mit der Anzahl der disjunkten Komponenten der Schnittmenge und mit der relativen Größe dieser Komponenten.

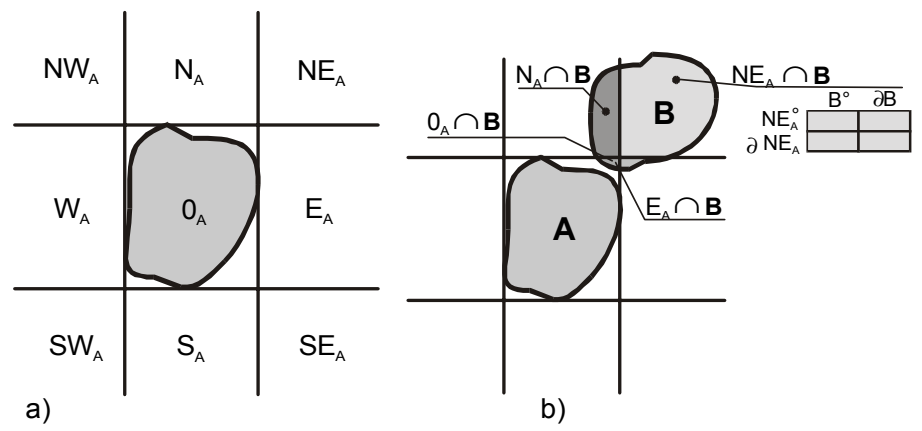

Abb. 30: DRM Beziehungen eines Geoobjektes: (a) Richtungsbeziehungen des Geoobjektes A, (b) Intersektionsmodell der Geoobjekte A und B.

Die genauere Beschreibung der Schnittmenge bietet nur dann zusätzliche Informationen über die Richtung, wenn mindestens zwei Elemente der (groben) DRM verschieden von 0 sind. Liegt das gesamte Primärobjekt in der Akzeptanzfläche einer Relation, ereicht die relative Größe der Schnittmenge ihr Maximum von 1.

$$
\operatorname{dir}_{R R}(A, B)=\left[\begin{array}{ccc}
\frac{\operatorname{area}\left(N W_{A} \cap B\right)}{\operatorname{area}(B)} & \cdot & \cdot \\
\cdot & \cdot & \operatorname{area}\left(S \dot{E}_{A} \cap B\right) \\
\cdot & \operatorname{area}(B)
\end{array}\right]
$$

\subsubsection{Unsicherheit}

Die Unsicherheit kann in jeder Komponente eines Geoobjektes enthalten sein. In der geometrischen Komponente können primär Lage und Form mit Unsicherheit behaftet sein 
(BurRough 1992). In der thematischen Komponente sind die Ungenauigkeiten beim Messen der quantitativen Attribute und die qualitativen Attribute selbst die Gründe der Unsicherheit. Räumliche Beziehungen zwischen Geoobjekten sind inhärent unscharf oder können unscharf ausgedrückt oder definiert werden. Die Unsicherheit bezüglich der Position kann auf die Unsicherheit bezüglich der Beziehungen übertragen werden.

Die Unsicherheit der Grenze kann zwei Gründe haben (vgl. ERWIG \& SchNEIDER 1997; Clementini \& Di Felice 1996):

- Ein Geoobjekt besitzt zwar eine scharfe Grenze, ihre Position und Form ist aber entweder unbekannt oder kann nicht genau vermessen werden. Die Unsicherheitsquellen sind einerseits Messungen, anderseits die digitale Repräsentation.

- Ein Geoobjekt besitzt eine Grenze, die von Natur her unscharf ist oder ihr Verlauf ist aus konzeptioneller Sicht nicht interessant, oder es sind nur die topologischen Beziehungen für den Benutzer von Bedeutung. Die Unsicherheit ergibt sich in diesem Fall aus der Natur des Geoobjektes.
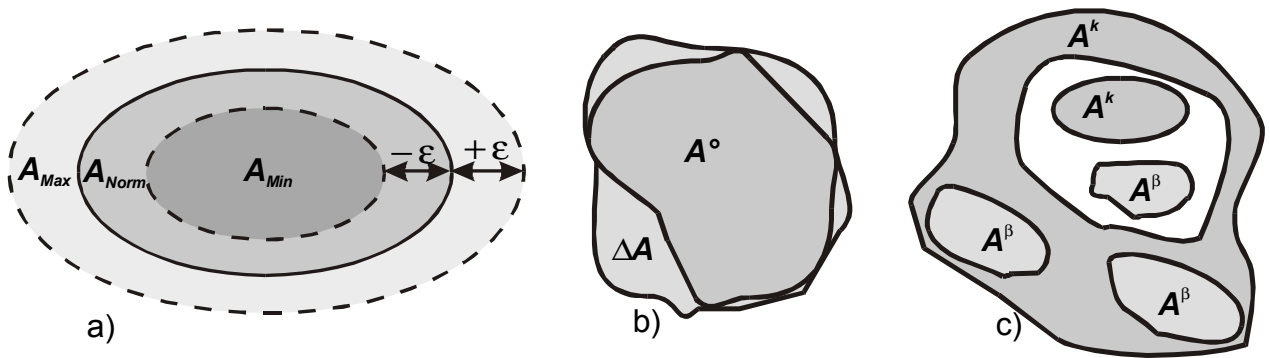

Abb. 31: Unsicherheitsmodelle der räumlich ausgedehnten Geoobjekte: (a) min-max Modell, (b) Modell der broad boundary, (c) Modell der vague area.

Angesichts dieser zwei Gründe unterscheidet man zwischen zwei Arten der Ungewissheit.

- Uncertainty (Unsicherheit) beschreibt die Ungewissheit von Position und Form (positional uncertainty) oder die Unmöglichkeit der genauen Messung (measurement uncertainty) eines sonst klar abgrenzbaren Objektes.

- Fuzziness (Unschärfe) beschreibt die innere Unschärfe eines Objektes, das mit Sicherheit eine Ausdehnung hat, das aber nicht eindeutig abgrenzbar ist.

Eine ungewisse Grenze kann mit einem der folgenden drei Verfahren behandelt werden. Fuzzy Modelle, die auf der Theorie der unscharfen Mengen beruhen, Wahrscheinlichkeitsmodelle, die von der Wahrscheinlichkeitstheorie und der Positions- und Messgenauigkeit ausgehen, und exakte Modelle, welche die Datenmodelle und Konzepte der Objekte mit scharfen Grenzen auf die Modelle mit unscharfen Grenzen und mit inhärenter Ungenauigkeit übertragen.

\subsubsection{Exakte Modelle}

Bei den exakten Modellen (Abbildung 31) geht man davon aus, dass die Unbestimmtheit der Grenze durch eine Fläche (Zone) angenähert werden kann, die das sicher Innere von dem sicher Äußeren trennt. Diese Art der Bewältigung der Unsicherheit ist dem Modell der topologischen Relationen in $\mathrm{Z}^{2}$ ähnlich. Diese Modelle benutzen die Datenmodelle, Strukturen und Algorithmen der geographischen Datenmodellierung, die für die scharfen Geoobjekte entwickelt wurden. 
Im Min-Max Modell (KRAUß 1998) wird jedes Objekt außer seines unscharfen Randes (Normalgeometrie) zusätzlich mit einem äußeren und einem inneren Rand eines Fehlerbandes $\varepsilon$ (Minimum- und Maximumgeometrie) versehen, innerhalb welcher der, wahre' Objektrand zu vermuten ist (Abbildung 31a). Für ein beliebiges Paar von zwei Objekten werden für alle diese Geometrien sämtliche topologische Beziehungen erfasst.

$$
R^{9}=\left(\begin{array}{ccc}
\operatorname{Rel}\left(\operatorname{Max}_{1}, \operatorname{Max}_{2}\right) & \operatorname{Rel}\left(\operatorname{Norm}_{1}, \operatorname{Max}_{2}\right) & \operatorname{Rel}\left(\operatorname{Min}_{1}, \operatorname{Max}_{2}\right) \\
\operatorname{Rel}\left(\operatorname{Max}_{1}, \operatorname{Norm}_{2}\right) & \operatorname{Rel}\left(\operatorname{Norm}_{1}, \operatorname{Norm}_{2}\right) & \operatorname{Rel}\left(\operatorname{Min}_{1}, \operatorname{Norm}_{2}\right) \\
\operatorname{Rel}\left(\operatorname{Max}_{1}, \operatorname{Min}_{2}\right) & \operatorname{Rel}\left(\operatorname{Norm}_{1}, \operatorname{Min}_{2}\right) & \operatorname{Rel}\left(\operatorname{Min}_{1}, \operatorname{Min}_{2}\right)
\end{array}\right)
$$

Max $=$ Maximalgeometrie, Min $=$ Minimalgeometrie, Norm $=$ Normalgeometrie, Rel - Relation

Clementini \& Di Felice (1996) beschreiben ein Modell für Objekte mit ausgedehntem (broad) Rand (Abbildung 31b). Ein Objekt in diesem Modell hat einen inneren und einen äußeren Rand (inner boundary, outer boundary). Beide sind geschlossene Kurven. Die durch den inneren Rand definierte Fläche ist in der mit dem äußeren Rand abgeschlossenen Fläche enthalten. Beide Ränder repräsentieren die Ungewissheit des Objektes in Form der minimalen und maximalen Ausdehnung.

Ein Objekt $A$ wird also von zwei einfachen Polygonen $A_{1}$ und $A_{2}$ gebildet, wobei $A_{1} \subseteq A_{2}$ und $\partial \mathrm{A}_{1}$ als innerer Rand (inner boundary) und $\partial \mathrm{A}_{2}$ als äußerer Rand (outer boundary) von $\mathrm{A}$ bezeichnet wird. Die broad boundary $(\Delta)$ erstreckt sich zwischen dem inneren und dem äußeren Rand: $\Delta \mathrm{A}=\mathrm{A}_{2}-\mathrm{A}_{1}{ }^{\circ}$. Exterior von $\mathrm{A}$ ist gleich der Differenz von $\mathbb{R}^{2}$ und $\mathrm{A}_{2}$, und closure von $\mathrm{A}$ wird als Vereinigung von A's interior und broad boundary definiert. Wenn $\mathrm{A}_{1} \subset \mathrm{A}_{2}$, dann ist $\Delta \mathrm{A}$ zweidimensional. Im Extremfall, wenn $\mathrm{A}_{1}=\mathrm{A}_{2}$, ist $\Delta \mathrm{A}$ eindimensional, d.h $\Delta \mathrm{A}=\partial \mathrm{A}$. Ist $\partial \mathrm{A}_{1} \cap$ $\partial \mathrm{A}_{2} \neq \varnothing$, dann ist $\Delta \mathrm{A}$ nicht homogen 2-dimensional und kann einen 1-dimensionalen Teil und mehrere Komponenten enthalten.

ERWIG \& SCHNEIDER (1997) haben ein Model der unsicheren (vague) Region entwickelt. Das unscharfe Objekt (vague region) $v$ wird in diesem Modell als ein Paar der disjunkten Regionen $(\mathrm{k}, \mathrm{b})$ modelliert (Abbildung 31c). Die erste Region, genannt auch kernel (k), beschreibt den sicheren Teil der unscharfen Region, d.h. den Teil, der bestimmt der Region angehört. Die zweite Region, genannt auch boundary (b), beschreibt den unscharfen Teil der Region, d.h. den Teil, über den wir nicht mit Sicherheit sagen können, ob er zur Region gehört, oder nicht. In diesem Modell muss der scharfe Teil des Objektes nicht vollkommen in dem unscharfen enthalten sein. Boundary muss nicht eindimensional sein. Kernel und boundary können benachbart sein, sie können Löcher wiederum mit einem oder beiden Teilen besitzen, so dass eine Hierarchie von kernel und boundary entstehen kann.

\subsubsection{Fuzzy Modelle}

Die fuzzy Modelle gehen über das Konzept der scharfen Geometrie der Grenze hinaus und modellieren diese als graduelle Veränderung mit multipler Zugehörigkeit innerhalb einer Zone der Unschärfe. Sie gehen von der fuzzy Mengentheorie und der fuzzy Topologie aus und stützen sich auf die ,fuzzy spatial algebra' (SCHNEIDER, M. 1999).

Unschärfe beschreibt nicht Unsicherheit im Sinne der Wahrscheinlichkeit, sondern die Ungenauigkeit des Konzeptes: ,...the vagueness represented by fuzziness is not the uncertainty of 
expectation. It is the vagueness resulting from the imprecision of meaning of a concept" (SCHNEIDER, M. 1999). Sie ist eine subjektive Größe im Gegenteil zur objektiven Wahrscheinlichkeit und behandelt vor allem lokale Unklarheit. Die Unschärfe stellt höhere Ansprüche an die Repräsentation und Analyse der Objekte (ALtman 1994). Außer den Geoobjekten mit gradueller Grenze kann die Unschärfe die räumliche Relation betreffen, wenn es zwischen zwei Objekten mehrere Beziehungen gleicher Art mit unterschiedlichem Zugehörigkeitswert gibt. Die Unschärfe einer räumlichen Beziehung kann durch das Konzept verursacht werden, auf dem sie basiert. Beispielsweise können verschiedene Benutzer verschiedene Relationen mit gleichem Namen bezeichnen (PAPADIAS et al. 1999).

Ein fuzzy Objekt ist eine fuzzy Menge, deren Domäne der zugrundeliegende Raum ist (USERY 1996). Ein fuzzy Objekt entsteht durch eine Erweiterung der Objekte mit scharfer Geometrie um das Konzept einer unscharfen Grenze: „Regions may not be precisely identified and boundaries generally exist as gradients and should appear as zones rather than lines. The implication then ist that a region is generally a fuzzy concept and a boundary is generally a fuzzy line" (LEUNG 1987). Das kann z.B. für das Polygon im 2D-Raum und die Linie im 1D-Raum vorgestellt werden. Ein Punkt mit sicherer Position besitzt hingegen keine Grenze.

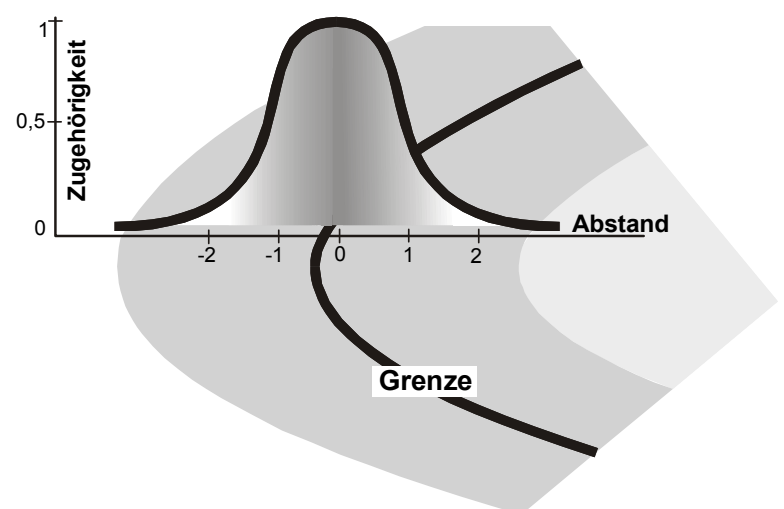

Abb. 32: Konzept der fuzzy-unscharfen Grenze eines räumlichen Objektes.

Es gibt zwei mögliche Interpretationen eines fuzzy Objektes (ALTMAN 1994). Es kann als ein Zugehörigkeitsgrad eines Punktes zur Region oder als Konzentration eines Attributs an einem Ort ausgedrückt werden.

Von Altman (1994) wird eine fuzzy Region als binäre Relation definiert, welche die Konzentration eines Objektattributs an dem Punkt beschreibt. LeUNG (1987) entwickelt ein System zur regionalen Klassifizierung anhand des Konzepts linguistischer Variablen und der Theorie der Möglichkeit (theory of possibility).

Eine Region besteht aus dem Kern und dem Rand. Der Rand ist eine Zone, die alle Punkte beinhaltet, deren Eigenschaften mehr oder weniger mit den Eigenschaften der Region übereinstimmen (Abbildung 32). Der ausgedehnte 2D Rand kann mit einem $\alpha$-Level eingeschränkt werden, um einen 1D Rand zu erzeugen. Der $\alpha$-Level Rand definiert eine $\alpha$ - Level Region $Z(\alpha)$. Ein unscharfer Rand kann mit einer oder zwei Kanten (edge) beschrieben werden. 
Die Grenze zwischen zwei Regionen $\mathrm{Z}$ und $\mathrm{W}$ wird von denjenigen Punkten gebildet, deren Eigenschaften mehr oder weniger mit der Eigenschaften der beiden Regionen übereinstimmen.

Eine Fuzzy Relation ist eine unscharfe Menge, die entweder die (scharfen) Elemente des kartesischen Produkts $\mathrm{X} \times \mathrm{Y}$ nach einer unscharfen Auswahlvorschrift bewertet, oder zwei Variablen mit unscharfen Werten (unscharfe Variablen) auf einem Grundbereich $\mathrm{X} \times \mathrm{Y}$ miteinander vergleicht (BOTHE 1995). DIJKMEIJER \& DE HoOP (1996) entwickelten ein Modell für topologische Beziehungen fuzzy overlap und fuzzy adjazenz. ALTMAN (1994) beschreibt die Ableitung der metrischen Beziehungen (Entfernung, Richtung) von zwei fuzzy Regionen.

\subsubsection{Zeit und zeitliche Beziehungen}

'All geographic phenomena have a spatio-temporal component as time and space constitute the frame of reference for our perceptions'. (CLARAMUNT \& THÉRIAULT 1995).

Die Zeit ist für die Analyse und das Verstehen räumlicher Phänomene unerlässlich. Die heutigen GIS Produkte erlauben die Berücksichtigung der zeitlichen Entwicklung nur als Gegenüberstellung von Momentaufnahmen des modellierten Phänomens. Eine Verbesserung dieses Zustandes durch die Eingliederung der zeitlichen Dimension in die Datenbank wird dringend benötigt. Die Erweiterung eines GIS um die zeitliche Fähigkeit erlaubt eine integrierte Analyse und Modellierung räumlicher Prozesse.

Die Zeit kann als ein universaler Pfeil dargestellt werden (YUAN 1997), der in einem vergangenen Punkt oder in der Unendlichkeit startet und sich über die Gegenwart uneingeschränkt in der Zukunft ausdehnt. Sie kann durch eine Metrik oder Ordnung beschrieben werden.

Die wichtigste Ordnungsrelation der Zeit ist die temporäre Aufeinanderfolge ' $<$ ' (die Abfolge; succession). Diese Relation ist irreflexiv, transitiv, linear, unbegrenzt und dicht (GALTON 1997). Ihre Domäne ist durch den 1-dimensionalen Charakter der Zeit bestimmt. Die Zeit ist gerichtet, der Vergangenheit (past) folgt die Gegenwart (presence) und dieser die Zukunft (future). Diese drei Klassen der zeitlichen Zustände sind nicht in ihren Eigenschaften gleich. Die Vergangenheit und die Zukunft sind zwei semi-offene Intervalle, während die Gegenwart punktförmig ist.

Die zeitlichen Objekte können grundsätzlich von zwei Typen sein: 0D Moment (instant), der wegen seiner Ähnlichkeit zum punktförmigen Objekt im Raum auch zeitlicher Punkt genannt wird, und 1D Intervall, auch temporäre Linie genannt. Der temporäre Punkt ist durch seine Position auf der temporären Achse, das temporäre Intervall durch die Position seiner beiden Endpunkte (Rand) definiert. Zusätzliche Eigenschaft des Intervalls ist seine Länge (Dauer).

Eine Änderung kommt immer dann vor, wenn eine der Objekteigenschaften eine neue Ausprägung annimmt. Die Änderung bedeutet die Tatsache der neuen Ausprägung, ein Ereignis. Ein Ereignis (event) wird als Menge der Prozesse in bezug auf das Ergebnis definiert, während ein Prozess eine Aktion in bezug auf den Mechanismus beschreibt (ClARAMUNT \& THÉRIAULT 1996). Ein Ereignis ist also eine Menge der zusammenhängenden Änderungen, die zu einem neuen Zustand der Entität führen. Es kann beobachtet werden, ohne den Mechanismus der Änderung zu kennen (CLARAMUNT et al. 1997). 
Ein Prozess, der zu einer Änderung führt, kann folgende Eigenschaften eines Objektes betreffen (CLARAMUnT et al. 1997):

- Existenz des Objektes

- Zugehörigkeit des Objektes zu einer Klasse (thematische Hierarchie)

- Wert eines thematischen, räumlichen oder zeitlichen Attributes (thematische, räumliche, zeitliche Änderung).

- thematische oder räumliche Beziehungen.

YUAN (1997) klassifiziert die Änderungen in Thematik, Raum und Zeit in 6 Klassen durch die Fixierung einer, Kontrolle der zweiten und Messung der dritten Komponente:

(1) Für eine gegebene Position kann das Auftreten und die Dauer der Ereignisse oder Attribute während einer Periode variieren. Die Position ist fixiert, Attribute werden kontrolliert, und die Zeit wird gemessen.

(2) Für eine gegebene Zeit kann ein Phänomen seine Eigenschaften von einer Position zur anderen ändern. Die Zeit ist fixiert, das Attribut kontrolliert, und die Position wird gemessen.

(3) Für eine gegebene Zeit kann sich eine Attributausprägung von einer Position zur anderen ändern. Die Zeit ist fixiert, die Position wird kontrolliert, und das Attribut wird gemessen.

(4) Für ein gegebenes Ereignis können seine Eigenschaften oder Prozesse während einer Periode von einer Position zur anderen geändert werden. Die Attribute werden fixiert, die Position wird kontrolliert, und die Zeit wird gemessen.

(5) Für eine gegebene Position können die Attribute von einer Periode zur anderen geändert werden. Die Position ist fixiert, die Zeit wird kontrolliert, und die Attribute werden gemessen.

(6) Für ein Ereignis kann seine Position von einer Periode zur anderen geändert werden. Das Attribut ist fixiert, die Zeit kontrolliert, und die Position wird gemessen.

\subsubsection{Zeitmodelle}

In den konzeptionellen Modellen treten unterschiedliche Zeittypen auf. Diese folgen nicht den Unterschieden der physischen Eigenschaften der Zeit, sondern ergeben sich aus der im konzeptionellen Modell festgelegten Abstraktion für eine konkrete Anwendung. FrANK (1998) beschreibt folgende Taxonomie der Zeitmodelle (Tabelle 6):

- Unterscheidung nach der temporären Dimension des Ereignisses: Ereignisse ohne (Zeitpunkte) und mit Dauer (Zeitintervalle)

- Unterscheidung nach der Prozessinterpretation: linear und zyklisch

- Unterscheidung nach dem Skalentyp: kontinuierliche Zeit gemessen mit Intervallskala und auf der ordinalen Zeitskala basierte Zeitsequenzinformationen

- Unterscheidung nach der Perspektive der Ereignisse: totale Ordnung, partielle Ordnung, verzweigte (branching) Zeit und mehrfache Kenntnisse über die Welt (real world time, transaction time)

Tab. 6: Konzeptionelle Zeitmodelle (nach FrANK 1998)

\begin{tabular}{|c|c|c|c|c|c|}
\hline & & Total order & Partial order & Branching & $\begin{array}{c}\text { Multiple } \\
\text { perspective }\end{array}$ \\
\hline \multirow[t]{2}{*}{ Linear } & Ordinal & $\begin{array}{c}\text { Single } \\
\text { experience }\end{array}$ & $\begin{array}{c}\text { Multiple } \\
\text { experience }\end{array}$ & \multirow{4}{*}{$\begin{array}{c}\text { Branching } \\
\text { Time }\end{array}$} & \multirow{4}{*}{$\begin{array}{l}\text { Time with } \\
\text { multiple } \\
\text { perspectives }\end{array}$} \\
\hline & Continuous & \multicolumn{2}{|c|}{ Continuous time } & & \\
\hline \multirow{2}{*}{ Cyclic } & Ordinal & \multirow{2}{*}{\multicolumn{2}{|c|}{ Cyclic time }} & & \\
\hline & Continuous & & & & \\
\hline
\end{tabular}




\subsubsection{Taxonomie der spatial-temporalen Prozesse}

Claramunt \& ThÉRIAUlt (1995) unterscheidet drei Typen des räumlich-zeitlichen Prozesses (Abbildung 33):
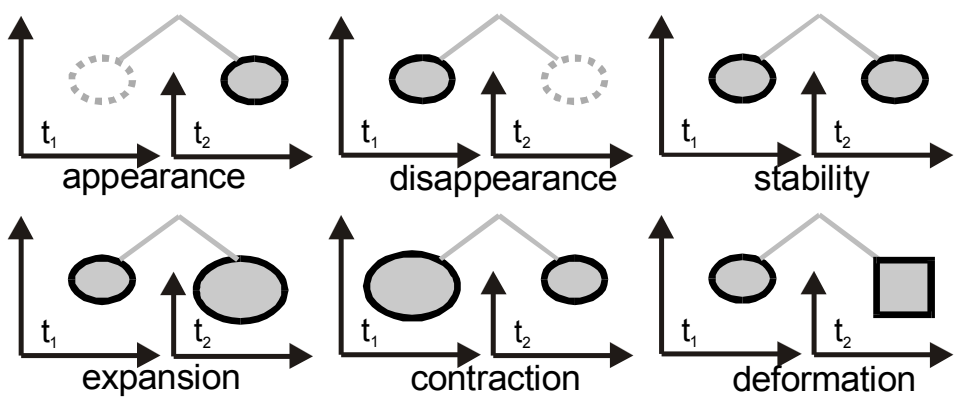

A)
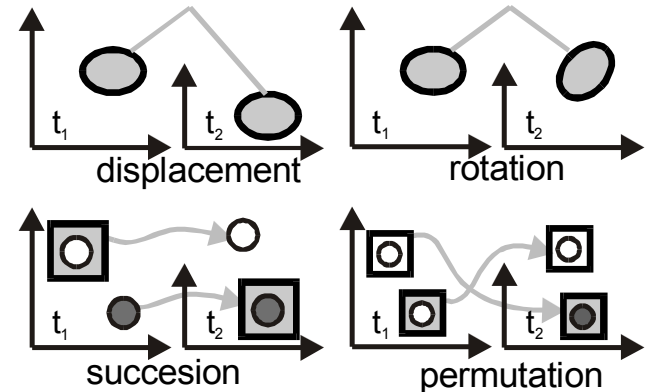

B)
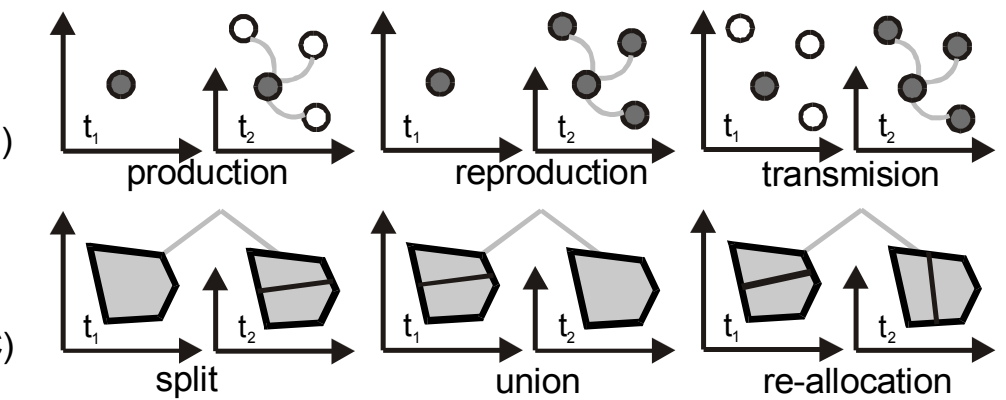

Abb. 33: Prozesse der Änderung von räumlichen Objekten (nach CLARAMUNT \& THÉRIAULT (1996) verändert).

(A) Die Entwicklungsprozesse der einzelnen Objekte beschreiben die individuelle Änderung der räumlichen Objekte. Ein beliebiger Prozess kann demnach als eine Aggregation von elementaren Prozessen aufgefasst werden.

(B) Die Prozesse, welche die funktionalen Beziehungen zwischen mehreren Objekten einschließen. Die funktionalen Beziehungen beschreiben die Abhängigkeitsbindungen, die explizit in der temporären Domäne modelliert werden (CLARAMUNT \& THÉRIAULT 1996).

(C) Die Prozesse der Entwicklung von räumlichen Strukturen (restucturing processes), welche die gleichzeitige Änderung von mehreren Objekten umfasst.

HoRnsBy \& EgENHOFER (1997) und Hornsby \& EgENHOFER (1998) entwickelten ein Modell zur qualitativen Repräsentation von Änderung, das explizit die Identität der räumlichen Objekte berücksichtigt (siehe Tabelle 7). 
Tab. 7: Identitätsbezogene Objektänderung nach HORNSBY \& EGENHOFER (1997).

\begin{tabular}{|c|c|}
\hline \multicolumn{2}{|c|}{$\begin{array}{l}\text { Operationen an einfachen Objekten } \\
\text { Grundoperationen }\end{array}$} \\
\hline create & bildet ein neues Objekt mit neuer Identität \\
\hline destruct & löscht ein existierendes Objekt \\
\hline continue & beschreibt die Fortsetzung eines Objektes mit gleicher Identität \\
\hline \multicolumn{2}{|c|}{ Kombination der einfachen Operationen } \\
\hline destruct und create & löscht ein vorhandenes Objekt und erzeugt ein neues mit neuer Identität \\
\hline reincarnation & löscht ein vorhandenes Objekt und erzeugt ein neues mit gleicher Identität \\
\hline issuing & ein Objekt wird von einem vorhandenen gebildet. \\
\hline spawn & $\begin{array}{l}\text { ein vorhandenes Objekt erzeugt ein neues Objekt mit eindeutiger, von der des ersten } \\
\text { Objekts verschiedener Identität }\end{array}$ \\
\hline netamorphose & ein vorhandenes Objekt erzeugt ein neues Objekt und beendet die eigene Existenz \\
\hline
\end{tabular}

Kombination der Objekte

eine Änderung tritt ein, wenn zwei Objekte zusammengefügt werden. Eine Kombination von Transition issue und Identitätsoperation (destruct und continue). merge
generate
mix
die ursprünglichen Objekte werden beim Zusammenfügen gelöscht, ein Objekt mit neuer Identität entsteht
die ursprünglichen Objekte werden beibehalten, ein Objekt mit neuer Identität entsteht eins der ursprünglichen Objekte wird gelöscht

Zusammengesetzte Objekte

aggregate

compoud

zwei einfache Objekte werden zu einem zusammengesetzten Objekt zusammenfügt mit einer neuen Identität, ohne daß sie die eigene Identität verlieren

eins oder mehrere Objekte werden einem zusammengesetzten Objekt beigefügt. Die einfachen Objekte und das zusammengestezte Objekt behalten ihre Identität

unite

zwei zusammengesetzte Objekte werden zu einem zusammengesetzten Objekt höherer Ordnung und neuer Identität zusammengefasst, ohne dabei die eigene

amalgamate Identität zu verlieren

amalgamate

zwei zusammengesetzte Objekte bilden ein neues zusammengesetztes Objekt. Die Teile von beiden ursprünglichen Objekten werden in einem neuen Objekt verknüpft und bekommen eine neue Identität

combine zwei zusammengesetzte Objekte bilden ein neues zusammengesetztes Objekt. Die Teilen von beiden ursprünglichen Objekten bleiben im neuen Objekt unverändert und behalten ihre Identität

\section{Zerlegung der Objekte}

Dieser Typ der Änderung (separate) ist von dem Typ issue verschieden, obwohl auch in diesem Fall ein neues Objekt von einem vorhandenen gebildet wird. Das neue Objekt verkleinert das ursprüngliche Objekt.

splinter

divide

secede

dissolve
Das ursprüngliche Objekt behält seine Identität, aus einem seiner Teile wird ein neues Objekt mit eigener Identität gebildet.

Das ursprüngliche Objekt wird in n Teile zerlegt und beendet seine Existenz

Ein Teil des zusammengesetzten Objektes trennt sich. Die Identität aller Objekte bleibt beibehalten.

Das zusammengesetzte Objekt wird in seine Teile zerlegt und beendet seine Existenz.

\subsubsection{Zeitliche Beziehungen}

Die Zeitbeziehungen drücken das Verhältnis von zwei (oder mehreren) Ereignissen zueinander aus. Sie hängen von dem zugrundeliegenden zeitlichen Konzept ab. Die zeitlichen Beziehungen ergänzen die Fülle der räumlichen Beziehungen um zwei Arten der Beziehungen:

- die räumliche Beziehung zweier zeitlicher Versionen eines Geoobjektes 
- die räumlichen Beziehungen von zwei (und mehreren) unterschiedlichen Geoobjekten in verschiedenen Zeitpunkten.

In der linearen Zeitdimension können die Ereignisse ohne zeitliche Ausdehnung mit Hilfe der Ordnungsrelationen $\{>, \geq,<, \leq,=, \cong\}$ verglichen werden. Die Beziehungen zwischen den Ereignissen mit Ausdehnung (Intervallen) können mit Hilfe von Allen's Intervallbeziehungen (ALLEN 1983) modelliert werden (Abbildung 34).

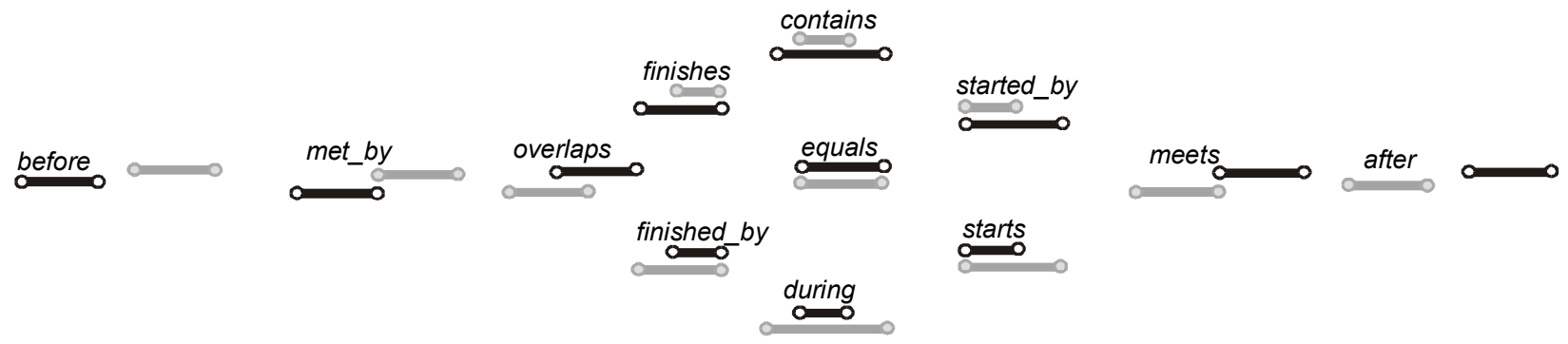

Abb. 34: Zeitbeziehungen des linearen Zeittyps (nach ALLEN 1983 verändert).

Im Modell der zyklischen Zeit benötigen die Beziehungen unterschiedliche Interpretation. FRANK (1998) definiert 3 Beziehungen, welche für das Zeitmodell angepasst werden müssen (Tabelle 8).

Tab. 8: Zyklische Zeitbeziehungen (nach FRANK 1998 verändert)

\begin{tabular}{|l|l|l|l|}
\hline 1 & $k$ equal $l$ & 7 & $k$ during $l$ \\
\hline 2 & $k$ meets $l$ & 8 & $k$ starts $l$ \\
\hline 3 & $k$ overlaps $l$ & 9 & $k$ finishes $l$ \\
\hline 4 & $k$ before $/$ after $l$ & End $(\mathrm{k})<$ Begin $(1)$ and $\operatorname{End}(1)<$ Begin $(\mathrm{k})$ \\
\hline 5 & $k$ overlap twice $l$ & End $(\mathrm{k})>$ Begin $(1)$ and $\operatorname{End}(1)>$ Begin $(\mathrm{k})$ \\
\hline 6 & $k$ complement $l$ & End $(\mathrm{k})=$ Begin $(1)$ and End $(1)=$ Begin $(\mathrm{k})$ \\
\hline
\end{tabular}

Das von HoRnsby \& EgENHOFER (1997) entwickelte 9-intersection Modell für die zeitlichen Beziehungen baut auf den Schnittmengen des Randes und des Inneren von zwei Zeitintervallen auf. Der Rand eines Zeitintervalls besteht aus dem Anfangs- und Endknoten $\left(\partial_{\mathrm{s}}, \partial_{\mathrm{e}}\right)$. Aus 512 möglichen Schnittmengen der Inhaltinvariante können 16 realisiert werden (Abbildung 35).
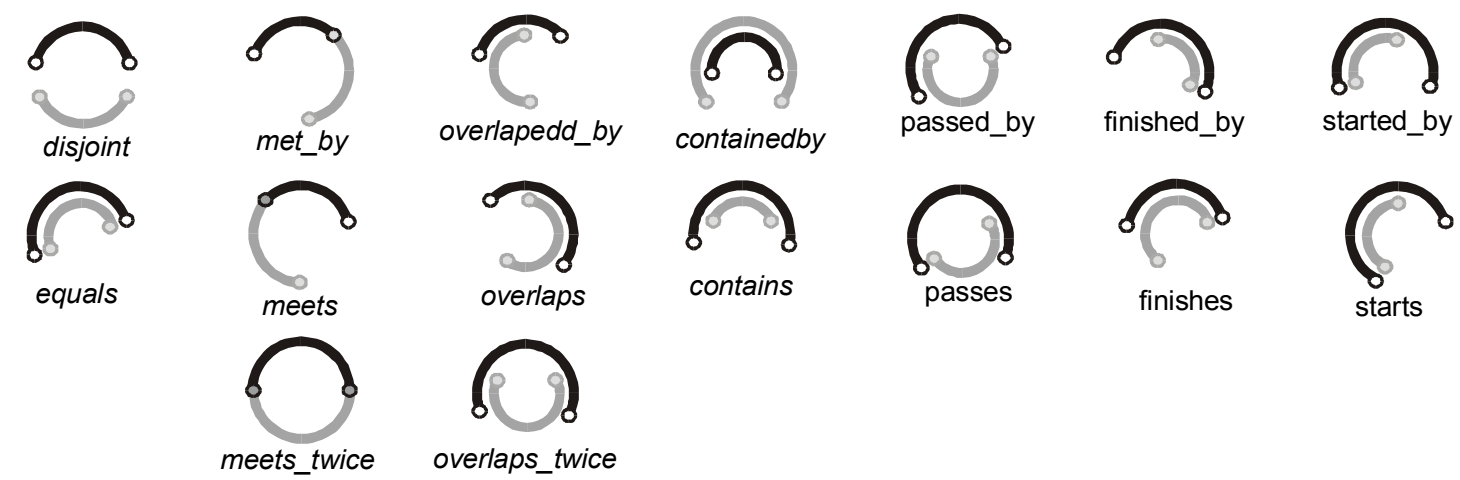

Abb. 35: Zeitbeziehungen im zyklischen Zeittyp (nach Hornsby \& Egenhofer (1997) verändert). 
Verzweigte Zeittypen beschreiben eine Situation, in welcher mehrere alternative künftige Entwicklungen auftreten können, beispielsweise bei der Planung unter Unsicherheit über die künftigen Zustände. ANGER et al. (1991) beschreiben neben den 13 Relationen für den linearen Fall, die auch im verzweigten Fall auftreten, noch 11 zusätzliche Relationen. Diese können dadurch entstehen, dass die Anfangs- und Endknoten der Intervalle in unterschiedlichen Zweigen liegen und daher unvergleichbar sind (Abbildung 36).
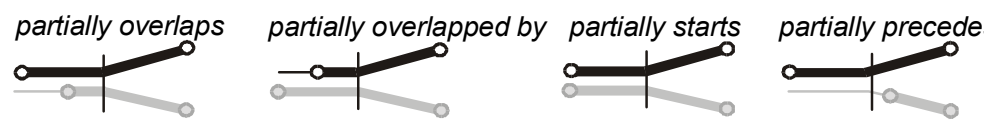

partially meets

partially met by
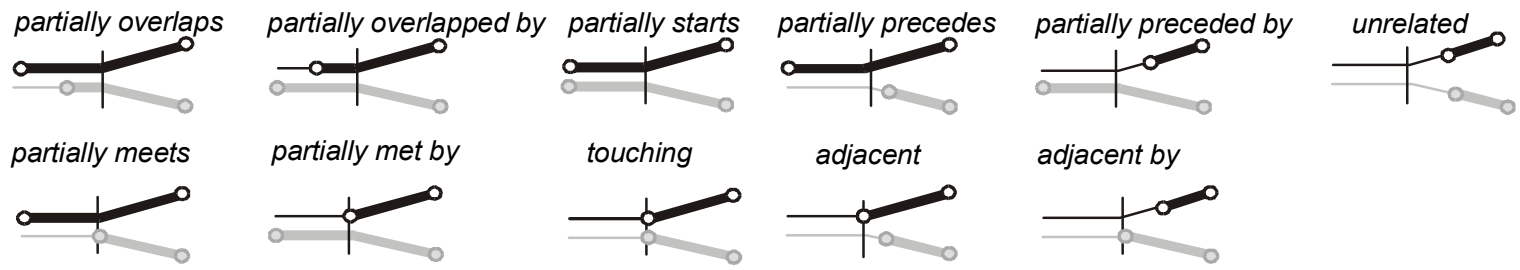

Abb. 36: Zusätzliche Zeitbeziehungen im verzweigten Zeittyp (nach ANGER et al. 1991 verändert). 


\section{Entscheidungstheorie}

\subsection{Entscheidung}

Die Entscheidung ist eine rational gesteuerte Auswahl einer von $n \geq 2$ möglichen Handlungsalternativen unter Berücksichtigung möglicher Umweltzustände und deren Eintrittswahrscheinlichkeiten (BUXMANN 1999). Eine Entscheidung unterscheidet sich von einer allgemeinen Wahlsituation durch den Einfluss der Handlungsalternativen auf die Zielerreichung (HÄNLE 1993). Nach JANSSEN (zitiert nach SCHWAPP 1995) ist ein Entscheidungsproblem eine Situation, in der ein Individuum oder eine Gruppe einen Unterschied zwischen dem gegenwärtigen und dem erwünschten Zustand festgestellt hat und in der: (i) dem Individuum oder der Gruppe alternative Vorgehensweisen zur Verfügung stehen, (ii) die Auswahl einer Vorgehensweise signifikante Auswirkungen auf diesen erkannten Unterschied haben kann und (iii) das Individuum oder die Gruppe a priori unsicher ist, welche Alternative gewählt werden soll.

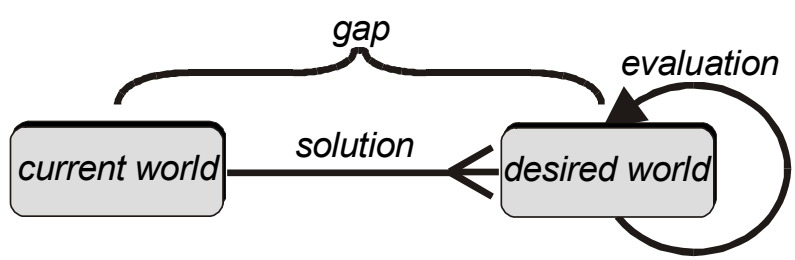

Abb. 37: Struktur eines Entscheidungsproblems nach dem 5 Komponenten Modell (in Anlehnung an CAMERON \& ABEL 1996).

Die Entscheidungsfindung setzt also eine als Problem empfundene Situation voraus, die auf verschiedene Weisen lösbar ist (Abbildung 37). Eine rationale Entscheidung geht von der Existenz der Zielvorstellungen aus, die als eine Schnittstelle zwischen dem Entscheidungsproblem und den möglichen Alternativen seiner Bewältigung beschrieben werden kann. In dem Prozess der Entscheidungsfindung kommt es auf die Erfahrung und Kreativität des Entscheidungsträgers (ET) an. Der ET muss sich der Restriktionen der Alternativen bewusst sein und die Konsequenzen der Alternativen abschätzen können. Anschließend kann er eine Alternative auswählen und somit eine Entscheidung treffen. Im Laufe der Realisierung der ausgewählten Alternative kommen allerdings weitere Entscheidungen zustande, die entweder vorher nicht vorhergesehen werden konnten oder die in der Präzisierung des Problems nicht berücksichtigt wurden.

\subsection{Entscheidung als Wissenschaft}

Die Entscheidungstheorie ist ,eine interdisziplinäre Forschungsrichtung, die sich mit der Formulierung und der Lösung von Entscheidungsproblemen beschäftigt" (BIETHAN et al. 1992 S.57). Sie ist eine interdisziplinäre Forschungsdisziplin, wobei jede der beteiligten Wissenschaften (Mathematik, Statistik, BWL, Psychologie etc.) die Entscheidung als gemeinsamen Erkenntnisgegenstand unter dem eigenen Blickwinkel beleuchtet. 
Die deskriptive Theorie erforscht die tatsächlich getroffenen Entscheidungen in bezug auf die Kriterien, sie beschreibt und erklärt die Wirklichkeit. Die Ergebnisse dieser empirischen Forschung geben eine Aussage über die Zulässigkeit der Alternativen und ihrer Konsequenzen. Das Ziel der deskriptiven Entscheidungstheorie ist es, ,empirisch gehaltvolle Hypothesen über das Verhalten von Individuen und Gruppen im Entscheidungsprozeß zu formulieren, mit deren Hilfe bei Kenntnis der jeweiligen Ausgangssituation Entscheidungen prognostiziert werden können " (LAUX 1998 S.14). Sie versucht, das Verhalten der Entscheidungsträger zu erklären um es prognostizieren zu können. Das menschliche Entscheidungsverhalten wird nach dem sog. SOR Paradigma untersucht. Der menschliche Organismus $(\mathrm{O})$ wird als eine Black-Box gesehen, die in ihrer Umwelt bestimmten Stimuli (S) ausgesetzt ist und durch Reaktionen (R) antwortet (BUXMANN 1999).

Die präskriptive Theorie (decision analysis) befasst sich primär mit der Rationalität der Entscheidungsfindung. Sie beschreibt nicht die Realität, sondern gibt Verhaltensempfehlungen für alternative Entscheidungssituationen (LAUX 1998). Sie zeigt, wie Entscheidungen 'rational' getroffen werden können. Der Inhalt der präskriptiven Entscheidungstheorie ist eine Analyse der Vorbereitung und Durchführung von rationalen Entscheidungen (DOCKNER 1999).

Rationale Entscheidungen sind immer mit dem Präferenzsystem (den Zielvorstellungen) des Entscheidungsträgers gekoppelt. In komplexen Entscheidungssituationen ist der Entscheidungsträger nicht in der Lage, seine Präferenz präzise zu formulieren. Die Methoden der präskriptiven Entscheidungstheorie versuchen, ihm bei dieser Aufgabe zu helfen. Der Begriff Rationalität betrifft nicht die Beurteilung des späteren Erfolgs oder Misserfolgs der gewählten Alternative, obwohl man durch rational gesteuerte Entscheidungen $\mathrm{zu}$ besseren Ergebnissen gelangen will. Man unterscheidet zwischen zwei Kriterien für rationale Entscheidungsprozesse. Die prozedurale Rationalität setzt eine 'rationale' Strukturierung des Entscheidungsprozesses voraus. Daneben fordert die Konsistenz der Entscheidungen die Vollständigkeit, Transitivität, Zukunftsorientierung und Unabhängigkeit von irrelevanten Alternativen.

\subsection{Entscheidung als Prozess}

Mit dem Begriff Entscheidung wird nicht nur die Auswahl einer optimalen oder mindestens guten Lösung verstanden, sondern auch ihre Vorbereitung und Rückkopplung in der Realisierungsphase. Die Entscheidungen werden im gesamten Verlauf des Entscheidungsprozesses getroffen. Problemformulierung, Alternativensuche oder Prognose über Ergebnisse verlangen eigene Entscheidungen (LAUX 1998). Die Entscheidung stellt somit einen Prozess dar, der aus Vorentscheidung und Endentscheidung besteht.

SIMONS (zitiert nach WERNER 1992) klassisches 4-Phasen-Schema zur Abbildung der Entscheidungsfindung besteht aus

- Intelligence: Problemerkennung und -formulierung

- Design: Erarbeitung von Lösungsalternativen

- Choice: Auswahl einer Alternative

- Review: Überprüfung der getroffenen Alternative 
Eine alternative Gliederung des Entscheidungsprozesses beschreibt GEISE (1997). Bei der Beobachtung der Umwelt (Märkte, System, ...) wird ein Problem erkannt. Zur Lösung des Problems werden in der Designphase Lösungen erarbeitet, die das Problem beheben können. Bei der Analyse der Alternativen werden unterschiedliche Methoden angewandt. Das Ergebnis dieses Schrittes ist die Aussage, welche Lösung der anderen vorzuziehen ist. Diese Entscheidung wird dann in der Umwelt verwirklicht. Der Erfolg der ausgewählten Alternative wird kontrolliert. Die Überwachung führt möglicherweise zum Erkennen eines neuen Problems. Der eigentliche Entscheidungsprozess, der von der präskriptiven Entscheidungstheorie unterstützt wird, geschieht auf den Stufen "Design" und "Analyse".

\subsection{Entscheidungsfindung als System}

Der Entscheidungsprozess kann als Interaktionsprozess zwischen zwei Systemen, einem Subjektsystem und einem Objektsystem, verstanden werden (BIETHAN et al. 1992).

Das Subjektsystem kann weiter zerlegt werden in:

- Zielsystem, das die verfolgten Ziele beschreibt;

- Informationssystem, das die zielorientierten Informationen über das Objektsystem ermittelt, und

- Entscheidungslogiksystem, das die Information zusammen mit den im Zielsystem vorgegebenen Prämissen verarbeitet und die durchzuführenden Aktionen ermittelt.

Das Objektsystem besteht aus folgenden Bestandteilen:

- Das Umweltsystem enthält alle durch den Entscheidungsträger nicht beeinflussbaren Bedingungskonstellationen.

- Das Aktionssystem enthält alle zur Verfügung stehenden Aktionsmöglichkeiten.

- Das Ergebnissystem ergibt sich aus dem Zusammentreffen von Aktions- und Umweltmöglichkeiten.

SCHNEEWEISS (1991) unterscheidet in einem Entscheidungsmodell ein Objekt- und ein Wertsystem. Das Objektsystem ,, beschreibt und strukturiert den durch Planung zu gestaltenden Sachverhalt" (SCHNEEWEISS 1991, S.54). Vor allem werden die Aktionsmöglichkeiten und ihre Konsequenzen beschrieben. Das Wertsystem wird als „System aller Präferenzrelationen innerhalb der Menge möglicher Objektsysteme" definiert. Dabei geht es nicht nur darum, die einzelnen Alternativen zu beurteilen. Auch die Gestaltung des Objektsystems ist Aufgabe des Wertsystems.

\subsection{Entscheidung als Modell}

Unter einem Modell versteht man üblicherweise ein zweckgerichtetes, vereinfachtes Abbild der Realität. Ein Entscheidungsmodell stellt „,das Ergebnis eines Versuches dar, die für wesentlich gehaltenen Elemente und Beziehungen einer als Problem empfundenen Handlungssituation in einer formalisierten Sprache so zu definieren, dass aus dem resultierenden Strukturkomplex die Problemlösung als logische Implikation abgeleitet werden kann" (BRETZKE 1980, S.8). 
SCHNEEWEISS (1991) beschreibt das Entscheidungsmodell als Gesamtheit von Objekt- und Wertsystem. Das Objektsystem allein stellt das Entscheidungsfeld dar, das aus einer Aktionenmenge (Menge der Handlungsalternativen A), einer Konsequenzenmenge bezogen auf die Zielgrößen des Entscheidungsträgers (Ergebnisse X) und einer Menge der Input-Output Relationen besteht. Die Konsequenzen der Alternativen werden in einer Konfiguration der Alternativen und Umweltzustände $(Z)$ nach einer Transformation $(A, Z) \rightarrow X$ abgeleitet. Die Ergebnisse werden durch ein Zielkriterium $K$ beurteilt, das zu einer Ordnung in der Ergebnismenge $X$ führt. Ein Entscheidungsfeld $F:=\{A, Z, X\}$ wird mit Hilfe eines Zielkriteriums zu einem Entscheidungsmodell M:= $\{\mathrm{F}, \mathrm{K}\}$ erweitert (SCHNEEWEISS 1991, S.73).

Das Zielkriterium kann durch eine Zielfunktion ersetzt werden. Im Allgemeinen versteht man unter Zielfunktion die formale Darstellung einer Entscheidungsregel (LAUX 1998). Eine Entscheidungsregel besteht aus einer Präferenzfunktion, die den Alternativen die Präferenzwerte zuordnet, und einem Optimierungskriterium, das angibt, welche Ausprägungen des Präferenzwertes angestrebt werden. Der Präferenzwert bringt somit den Grad der Zielerreichung zum Ausdruck. Das Optimierungskriterium kann den Präferenzwert maximieren (bzw. minimieren), oder es strebt eine Mindest- oder fixierte Ausprägung des Präferenzwertes an. Im Gegensatz dazu schränkt ein Entscheidungsprinzip den Bereich der Präferenzfunktion ein, jedoch verbleiben noch mehrere, zwischen welchen noch gewählt werden muss. Somit führt das Entscheidungsprinzip nicht zur eindeutigen Lösung, sondern zu einer Menge möglicher Lösungen.

Es werden allgemeine Entscheidungsmodelle, sog. A-Modelle, und konkrete Entscheidungsmodelle (K-Modelle) unterschieden. Für die Lösung eines konkreten Problems muss ein A-Modell in ein K-Modell überführt werden, indem seine Modellparameter festgelegt werden (LAUX 1998).

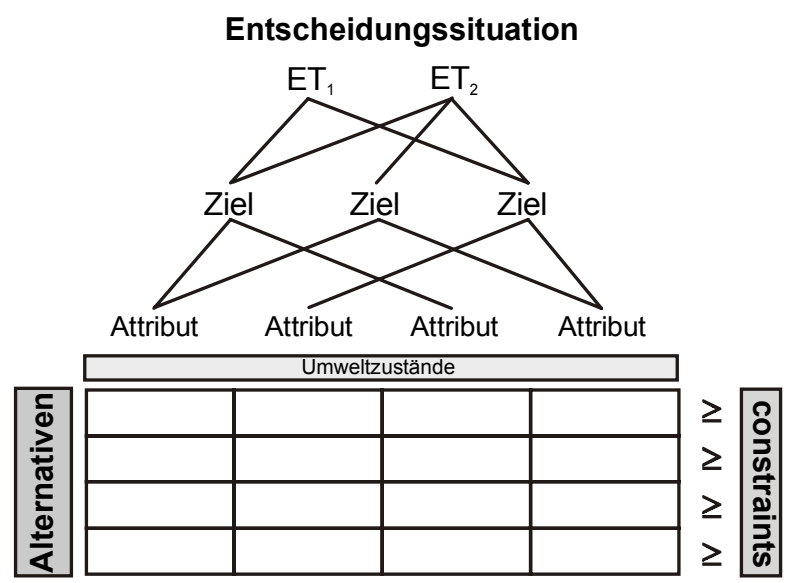

Abb. 38: Struktur einer Entscheidungssituation.

Das Grundmodell der Entscheidungstheorie (Abbildung 39) ist eine Darstellung einzelner Elemente des Entscheidungsmodells. Es besteht aus einer Ergebnismatrix mit den Alternativen in der Vorspalte und den Umweltzuständen in der Kopfzeile und einer Zielfunktion. In Entscheidungssituationen mit mehreren Entscheidungskriterien können diese in die dritte Dimension der Matrix aufgenommen werden. Im Fall einer Entscheidung unter Risiko werden die Umweltzustände durch die Wahrscheinlichkeiten ihres Auftretens ergänzt. 
Das Grundmodell soll folgenden Grundvoraussetzungen genügen (BIETHAN et al. 1992, GöTZE \& BLOECH 1995):

- Der Entscheidungsträger ist sich aller Handlungsalternativen bewusst.

- Alle möglichen Umweltzustände sind bekannt (zusätzliche Informationen über die Umwelt kann sich der Entscheidungsträger nicht beschaffen).

- Der Entscheidungsträger kann die Umweltzustände nicht beeinflussen.

- Jede Aktionsvariable muss mit jeder Umweltvariable kombinierbar sein und jeder Kombination kann ein Ergebnis zugewiesen werden.

- Bezüglich der ermittelten Ergebnisse existiert eine Präferenzfunktion des Entscheidungsträgers.

- Die getroffene Wahl kann man nicht wieder korrigieren.

- Die Alternativen müssen sich gegenseitig ausschließen.

In jedem Fall ist eine Alternative $\mathrm{zu}$ wählen (auch die Unterlassungsalternative muss berücksichtigt werden, falls eine Handlung nicht gefordert wird).

Abb. 39: Grundmodell der Entscheidungstheorie.

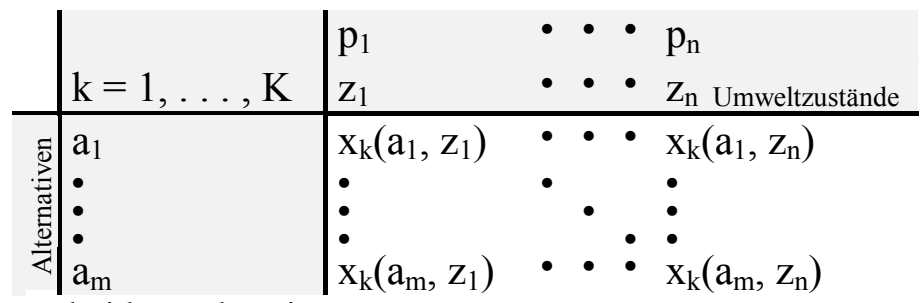

Nicht immer sind alle Voraussetzungen erfüllt, das Grundmodell der Entscheidungstheorie ist nur ein Gerüst, das weiter verfeinert werden muss. Die Vorteile dieses Gerüsts liegen in der Neutralität gegenüber den unterschiedlichsten Anwendungsfällen und in seiner flexiblen Ausbaufähigkeit.

Bei einer konkreten Anwendung müssen die Grundbegriffe präzisiert werden. Es muss geklärt werden, welche die relevanten Alternativen sind, wie die Ergebnisse ermittelt werden, wovon sie abhängen, welche Umweltzustände mit welcher Wahrscheinlichkeit vorkommen können und welche Werte für die Ausprägungen der Kriterien möglich sind (LAUX 1998). Die Kritik des Grundmodells der Entscheidungstheorie richtet sich vor allem auf die Unvollständigkeit des Modells: Es wird nur die letzte Phase des Entscheidungsprozesses, die Alternativenauswahl, in den Ansatz einbezogen. Die anderen Phasen, wie die Problemerkennung oder die Suche nach Informationen werden außer Betracht gelassen. Der Entscheidungsträger wird als geschlossenes System betrachtet, das Zustandekommen der Entscheidungsprämissen ebenso wenig berücksichtigt wie die vielseitigen wechselseitigen Beziehungen zwischen dem Entscheidenden und seiner Umwelt (WERNER 1992).

Die Darstellung des Grundmodells ist aber bei vielen Alternativen oder Zuständen sehr aufwendig, bei unendlicher Anzahl der Alternativen ganz unmöglich. Es gibt noch zwei weitere Modelltypen: graphische Modelle und mathematische Modelle. Diesen Modellen liegen die Annahme der kontinuierlichen Entscheidungsvariablen und sehr viele Alternativen zu Grunde. Die graphische Darstellung versagt im Falle von mehr als 2 Zielgrößen. 


\subsection{Präferenz}

Die Präferenz ist eine Einsicht des ETs, aufgrund welcher er eine Alternative einer anderen vorzieht oder beide als gleich ansieht (vgl. EISENFÜHR \& WEBER 1999). Zur Lösung eines Entscheidungsproblems wird die Präferenz abgebildet und gemessen. Eine zukunftsorientierte Entscheidung zeichnet sich dadurch aus, dass die Präferenz eines ETs aus einer Bewertung der Konsequenzen der Alternativen abzuleiten ist.

Auf Mengen der Entscheidungsalternativen werden durch binäre Relationen Ordnungsstrukturen eingeführt. Äquivalenz- und Ordnungsrelationen stellen die Grundlage der mathematischen Entscheidungstheorie dar. Sie werden auch Indifferenz- bzw. Präferenzrelationen genannt.

Eine Präferenzrelation (auch als schwache Ordnung, oder schwache Präferenz bezeichnet) ist eine Relation $(\mathrm{R} \subseteq \mathrm{X} \times \mathrm{X})$, die vollständig (linear) und transitiv ist. Die Vollständigkeit einer Relation impliziert ihre Reflexivität. Eine schwache Ordnungsrelation kann in eine numerische Beziehung (mittels Wertfunktion) übertragen werden. Die Ordnung nennt man deswegen „schwach“, weil es immer noch Alternativen geben kann, die als gleichrangig angesehen werden (SCHNEEWEISS 1991, S.93). Eine starke Ordnung bezüglich der Alternativen liegt dann vor, wenn es keine zwei Alternativen gibt, zwischen denen der Entscheidungsträger indifferent ist.

Bei den Entscheidungen höherer Dimensionalität stellt sich das Problem der Aggregation der auf einzelne Zielkriterien bezogenen Präferenzrelationen zu einer Präferenzrelation über die Handlungsalternativen. Liegt nicht die Dominanz einer Alternative bezüglich allen Attributen vor, muss die Wichtigkeit der Dimensionen miteinander abgewogen werden. Diese Trade-off (Austausch-)Beziehungen werden durch den Übergang von der ordinalen zu der metrischen Skalierung der Kriterien und die Verknüpfung dieser auf Einzelkriterien bezogenen Nutzenmaße zu einer Gesamtnutzenfunktion erleichtert (BUXMANN 1999).

Im Laufe des Entscheidungsprozesses ist die Präferenz des Entscheidungsträgers an mehreren Stellen gefragt. Die Äußerung der Präferenz eines Entscheidungsträgers (ET) ist immer dann notwendig, wenn eine ,Mehrdimensionalität' in der Entscheidungssituation vorkommt.

- Die Höhenpräferenz ist eine Präferenz, mit welcher die Anordnung von Handlungsalternativen, die ein Ziel in unterschiedlichem Ausmaß erfüllen, erst möglich ist.

- Die Artpräferenz ordnet Alternativen im Fall von mehreren Zielen oder Kriterien an, die zumindest teilweise in Konflikt stehen und daher von den Alternativen in unterschiedlichem Ausmaß erfüllt werden.

- Die Zeitpräferenz ordnet die Alternativen in bezug auf die Zeitpunkte, zu welchen die Konsequenzen der Alternativen auftreten.

- Die (Un)Sicherheitspräferenz erlaubt die Anordnung der Alternativen, bei welchen die Konsequenzen von dem künftigen Umweltzustand abhängen und daher nicht mit Sicherheit bekannt sind.

- Die Gruppenpräferenz versucht, die individuellen Alternativenanordnung aller Gruppenglieder miteinander zu einer Gruppenanordnung zu verknüpfen. 


\subsubsection{Höhenpräferenz}

$A$ bezeichnet die Menge der Alternativen einer Entscheidungssituation, die anhand der Präferenz des Entscheidungsträgers geordnet werden sollen. Ist die Präferenzrelation vollständig und transitiv (d.h. es liegt eine schwache Ordnung vor) so ist sie numerisch durch eine Funktion $v: A \rightarrow \mathbb{R}$ darstellbar, welche die Ordnungsrelation $\succsim$ in die numerischen Beziehungen $>$ und $=$ überträgt $\left(v\left(a^{\prime}\right) \geq v\left(a^{\prime \prime}\right)\right.$ ist äquivalent zu a' $\left.\succsim a^{\prime \prime}\right)$ (SCHNEEWEISS 1991). Diese Funktion wird auch Wertfunktion $v$ genannt. Eine Wertfunktion ist bis auf eine positive und lineare Transformation eindeutig.

Diese Wertfunktion definiert nur eine Ordnung der Alternativen, nicht jedoch die Stärke der Präferenz zwischen den Alternativen. Sie wird auch als ordinale (nicht-messbare) Wertfunktion bezeichnet. Im Gegensatz dazu erlaubt eine messbare Wertfunktion (measurable value function) auch eine Abbildung der Stärke der Präferenz. Um das zu ermöglichen, muss der ET auch eine Präferenz hinsichtlich der Übergänge zwischen den Alternativen haben. Der ET muss die Übergänge (Unterschiede der Konsequenzen zweier Alternativen) miteinander vergleichen können. Die Präferenz hinsichtlich der Übergänge muss wiederum vollständig und transitiv sein.

\subsubsection{Artenpräferenz}

Im Fall von mehreren Entscheidungskriterien (Attributen) kann, unter gleichen Voraussetzungen wie im Fall eines Kriteriums, eine multiattributive Wertfunktion hinsichtlich mehrfacher Ziele abgebildet werden. Ihre additive Form setzt voraus, dass für jedes Attribut eine Wertfunktion existiert (eindimensionale Wertfunktion, Einzelwertfunktion). Die multiattributive Wertfunktion wird dann als Summe der gewichteten Einzelwertfunktionen definiert. Die rationale Begründung des additiven Wertmodells setzt die Unabhängigkeit der Bewertung aller Attribute voraus. Eine Präferenzunabhängigkeit zwischen zwei beliebigen Alternativen ist dann gegeben, wenn die Präferenz einer Alternative gegenüber einer anderen hinsichtlich eines Attributes (Zieles) unabhängig davon erhalten bleibt, welche Werte die anderen Attribute annehmen (EISENFÜHR \& WeBER 1999). Die Konsequenz der Präferenzunabhängigkeit ist die Existenz einer Einzelwertfunktion für jedes Zielattribut. Liegt die schwache Präferenzunabhängigkeit nicht vor, wird man versuchen, die Menge der Attributsausprägungen einzuschränken oder Attribute zusammenzufassen. Ein additives Modell (der nicht-messbaren multiattributiven Wertfunktion) ist nur in dem Fall gültig, wenn jedes Attribut (von allen anderen Attributen) präferenzunabhängig ist (einfache Präferenzunabhängigkeit) und wenn jede Teilmenge der Attribute von der jeweiligen Komplementärmenge präferenzunabhängig ist (wechselseitige oder starke Präferenzunabhängigkeit).

Für die Gültigkeit des additiven Modells auch für die messbare Wertfunktion wird zusätzlich die Bedingung der Differenzunabhängigkeit verlangt. Nach dieser Bedingung muss auch der Übergang zwischen zwei Alternativen präferenzunabhängig sein, d.h. die Präferenz bezüglich eines Übergangs zwischen zwei Alternativen in einem Attribut ist unabhängig von den Ausprägungen aller anderen Attribute.

\subsubsection{Risikopräferenz}

Bei Entscheidungen unter Risiko hängt die Konsequenz einer Alternative davon ab, welcher der möglichen Umweltzustände tatsächlich auftritt. Die Erwartung des Auftretens der möglichen 
Umweltzustände muss quantitativ messbar sein. Die Nutzenfunktion, wie die Wertfunktion in Risikosituationen bezeichnet wird, spiegelt in diesem Fall nicht allein die Konsequenz wider, sondern auch die Risikoeinstellung des ETs (EISENFÜHR \& WEBER 1999, LAUX 1998). Dabei werden weitere Bedingungen (Axiome) gestellt, unter deren Erfüllung eine Nutzenfunktion existiert (abgeleitet werden kann) und darstellbar ist. Neben der vollständigen Ordnung geht die Präferenzfunktion auch von der Stetigkeit aus.

Die additive Form der Funktion verlangt die Erfüllung der Bedingung der Unabhängigkeit (Substituierbarkeit). Bei Entscheidungen mit mehreren Zielen versucht man, die multiattributive Nutzenfunktion additiv zu zerlegen. Die Bedingung der Präferenzunabhängigkeit über die Attribute ist aber für die Gültigkeit eines additiven Modells nicht ausreichend. Die neben der Konsequenzpräferenz in einer Nutzenfunktion enthaltene Risikoeinstellung wird durch das Konzept der Nutzenunabhängigkeit berücksichtigt.

\subsubsection{Zeitliche Präferenz}

Das intertemporale Entscheidungsproblem ist dem Entscheidungsproblem unter mehrfacher Zielsetzung ähnlich. Eine intertemporale Wertfunktion, unter Gültigkeit der Bedingungen für eine Präferenzrelation und wechselseitige Präferenzunabhängigkeit, kann durch eine additive Wertfunktion abgebildet werden. Ist auch die Bedingung der Differenzunabhängigkeit erfüllt, so ist diese Funktion auch messbar. Für jede Zeitperiode kann eine eigene Einzelwertfunktion definiert werden. Das additive Modell kann mit weiteren Axiomen vereinfacht werden.

Das Diskontierungsmodell (EISENFÜHR \& WEBER 1999) benötig neben den oben erwähnten Bedingungen auch noch die Bedingung der Stationarität. Stationarität ist definiert als Invarianz der Präferenz gegenüber einer Verschiebung. Das Diskontierungsmodell geht von einer für alle Perioden identischen Einzelwertfunktion aus. Ein weiteres Modell der Weiteraxiomatisierung des additiven Modells ist das Modell von Harvey. Dieses Modell berücksichtigt zusätzlich die Bedingung der Ungeduld und der Konstanz der Präferenz bei Streckung. Nach der ersten Bedingung wird die früher aufgetretene Konsequenz als besser angesehen. Die zweite besagt, dass die Präferenz bei einer Streckung der Zeitreihe invariant bleibt. Beide Modelle stellen Spezialisierungen des additiven Modells dar.

\subsubsection{Gruppenpräferenz}

Die Aggregation der Präferenzvorstellungen aller Mitglieder einer Gruppe scheint dem Problem der Entscheidung bei mehreren Zielen ähnlich zu sein. Dieses Vorgehen setzt jedoch voraus, dass die Stärke der Präferenz eines Gruppenmitglieds mit der eines anderen Gruppenmitglieds verglichen werden kann. Die Möglichkeit eines interpersonellen (kardinalen) Wertvergleichs ist jedoch nach Meinung vieler Forscher nicht gegeben (EISENFÜHR \& WEBER 1999). Wird diese Möglichkeit angenommen, so wird im weiteren die Präferenzunabhängigkeit und die ordinale positive Assoziation vorausgesetzt. Wird aber diese Möglichkeit verneint, dann können nur die ordinalen Präferenzen der Gruppenmitglieder zu einer ordinalen Gruppenpräferenz aggregiert werden. 


\subsubsection{Outranking}

Das Outranking (Prävalenzsatz) bezeichnet eine Entscheidungstechnologie in der Situation mit unvollständiger oder widersprüchlicher Information.

Die klassische Entscheidungstheorie geht von einer Ordnungsrelation aus: zwei Handlungsalternativen sind demnach vergleichbar und transitiv. Der Entscheidungsträger ist in der Lage, im Vergleich zweier Alternativen eine Alternative der anderen vorzuziehen oder sie als indifferent $\mathrm{zu}$ bezeichnen. Wenn er eine Alternative a der Alternative b strikt vorzieht, und die Alternative $\mathrm{b}$ wird der Alternative $\mathrm{c}$ strikt vorgezogen, so muss auch a der Alternative $\mathrm{c}$ strikt vorgezogen werden.

Das Outranking geht davon aus, dass diese Bedingungen in einer konkreten Entscheidungssituation nicht erfüllt werden müssen. Die Präferenz nach dem Outranking wird in vier Grundsituationen modelliert, die beim Vergleich von zwei Alternativen vorkommen können.

\begin{tabular}{|c|c|c|}
\hline strikte Präferenz & $\mathrm{aPb}$ & $\begin{array}{l}\text { Die Alternative a wird der Alternatve } b \text { strikt } \\
\text { vorgezogen. }\end{array}$ \\
\hline Indifferenz & $\mathrm{aIb}$ & $\begin{array}{l}\text { Die Alternativen a und } \mathrm{b} \text { werden als indifferent } \\
\text { angesehen. }\end{array}$ \\
\hline schwache Präferenz & $\mathrm{aQb}$ & $\begin{array}{l}\text { Die Alternative } b \text { wird der Alternative a nicht strikt } \\
\text { vorgezogen, es ist aber unmöglich zu sagen, ob die } \\
\text { Alternative a der Alternative b strikt vorgezogen wird } \\
\text { oder ob a indifferent zu b ist. }\end{array}$ \\
\hline
\end{tabular}

Man versucht, die Gründe einer Präferenz einer Alternative gegenüber einer anderen mit den Gründen zu vergleichen, die das Gegenteil behaupten. Sind die Gründe dafür, dass die Alternative a der Alternative $b$ vorgezogen wird, stärker als die Gründe dafür, dass die Alternative $b$ der Alternative a vorgezogen wird, dann dominiert die Alternative a die Alternative b im Sinne der Outranking-Relation. Die Outranking-Relation ist weder vollständig noch transitiv.

\subsection{Multi - Criteria Analyse}

Das Ziel eines mehrkriteriellen Bewertungsverfahren besteht darin, aus einer Kriterienmenge, die eine Alternativenmenge hinsichtlich verschiedener Aspekte mehrdimensional beschreibt, eine Präferenzordnung für die Alternativen zu definieren.

Die Methoden der Multi-Criteria-Entscheidungen können in Entscheidungssituationen angewendet werden, in welchen mehrere Ziele angestrebt werden, zwischen welchen oft Konflikte auftreten und Konsequenzen der Alternativen in diversen Messeinheiten ausgedrückt werden können (HwANG \& YOON 1981).

Die Multi-Criteria Probleme werden üblicherweise in zwei Klassen eingeteilt (HwANG \& YoON 1981, ZIMMERMANN \& GUTSCHE 1991). 
- Multi-Attribut - Entscheidungen - Multi Attribute Decision Making (MADM)

- Multi - Objective - Entscheidungen - Multi Objective Decision Making (MODM)

Bei den MADM-Verfahren ist die Menge der zulässigen Alternativen diskret und vorbestimmt. Man spricht von diskreten Lösungsräumen. Jede Alternative wird hinsichtlich ihrer Attribute beurteilt. Die Attribute stellen in der Sprache des MADM die Ziele des Entscheidungsträgers dar.

Bei den MODM-Verfahren ist die Menge aller Alternativen nicht explizit vorbestimmt, die Zulässigkeit einer Alternative ist mit der Erfüllung vorgegebener Nebenbedingungen gegeben. Die Menge der zulässigen Alternativen enthält so grundsätzlich unendlich viele Elemente. Deshalb spricht man auch von stetigen Lösungsräumen. Die Ziele werden durch klare, quantifizierbare Zielfunktionen (objectives) definiert. Damit kann jeder Alternative bezüglich jedes Ziels ein bestimmter Wert zugeordnet werden.

".. The thrust of these models is to design the 'best' alternative by considering the various interactions within the design constraints which best satisfy the DM by way of attaining some acceptable levels of a set of some quantifiable objectives.." (HWANG \& YOON, 1981 S.3).

Da alle vorgegebenen Zielfunktionen gleichzeitig zu optimieren sind, bezeichnet man MODMModelle auch als Vektoroptimierungsmodelle. Die Gemeinsamkeiten der Modelle sind :

- Menge der quantifizierbaren Zielfunktionen (objectives)

- Menge der angegebenen Bedingungen

- Prozess der Berücksichtigung des Trade-Off

Tab. 9: Vergleich der MADM und MODM Verfahren (nach Hwang \& Yoon 1981).

\begin{tabular}{|l|l|l|}
\cline { 2 - 3 } \multicolumn{1}{c|}{} & \multicolumn{2}{c|}{ MADM } \\
\hline Kriterien (criteria) & Attribute (attributes) & Ziele (objectives) \\
\hline Ziele (objective) & implizit & explizit \\
\hline Attribute & explizit & implizit \\
\hline Bedingungen & eingearbeitet in Attribute & aktiv \\
\hline Alternativen & diskret und abzählbar & unendlich, stetig \\
\hline Interaktion mit ET & wenig & oft \\
\hline Verwendung & Auswahl / Bewertung & Design \\
\hline
\end{tabular}

Neben den klassischen MADM- Ansätzen und den MODM- Methoden unterscheidet man noch entscheidungstechnologische Ansätze (decision aid). Diese sind Methoden und Instrumente zur Informationsverarbeitung, mit denen der Entscheidungs- oder Problemlösungsprozess effizienter gestaltet werden kann (ZIMMERMANN \& GUTSCHE 1991, S.26). Effizienter bedeutet dabei, dass das Ausmaß der Zielerreichung unter Berücksichtigung des Ressourceneinsatzes gesteigert wird. Bei diesen Methoden werden auch unscharfe, unvollständige oder gar widersprüchliche Informationen (über Attribute und Handlungsalternativen) in den Entscheidungsprozeß einbezogen. Die Entscheidung wird ferner dem Entscheidungsträger überlassen. Nur wenn es der Entscheidungsträger ausdrücklich wünscht, wird ihm eine 'optimale' oder 'optimierende' Entscheidung vorgeschlagen. 
Tab. 10: Klassifikation von MADM - Methoden (In Anlehnung an HwANG \& YOON 1981, ZIMMERMANN \& GUTSCHE 1991).

\begin{tabular}{|c|c|c|c|}
\hline & Art der Information & $\begin{array}{c}\text { wesentliche Eigenschaft } \\
\text { von Informationen }\end{array}$ & Gruppen von Verfahren \\
\hline \multirow{3}{*}{\multicolumn{2}{|c|}{ keine Informationen }} & & Dominanz - Strategie \\
\hline & & & Maximin - Strategie \\
\hline & & & Maximax - Strategie \\
\hline \multirow{15}{*}{ 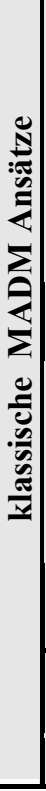 } & \multirow{12}{*}{$\begin{array}{l}\text { Informationen über } \\
\text { Attribute }\end{array}$} & \multirow{2}{*}{\begin{tabular}{|l}
$\begin{array}{l}\text { Anspruchsniveaus } \\
\text { (standard level) }\end{array}$ \\
\end{tabular}} & konjunktives Vorgehen \\
\hline & & & disjunktives Vorgehen \\
\hline & & \multirow{4}{*}{ ordinale Information } & lexikographische Methode \\
\hline & & & \begin{tabular}{|lll} 
lexikographische & Methode & mit \\
Halbordnung & & \\
\end{tabular} \\
\hline & & & Aspektweise Elimination \\
\hline & & & Permutations-Methode \\
\hline & & \multirow{5}{*}{ kardinale Information } & Lineare Zuordnungsmethode \\
\hline & & & Einfache additive Gewichtung \\
\hline & & & Hierarchische additive Gewichtung \\
\hline & & & Electre \\
\hline & & & Topsis \\
\hline & & $\begin{array}{|lll|}\text { Marginale } & \text { Rate } & \text { der } \\
\text { Substitution } & & \\
\end{array}$ & Hierarchische Tradeoffs \\
\hline & \multirow{3}{*}{$\begin{array}{l}\text { Informationen über } \\
\text { Alternativen }\end{array}$} & \multirow[t]{2}{*}{ Paarweise Präferenz } & Linmap \\
\hline & & & Interaktive SAW Methode \\
\hline & & Paarwise Proximity & MDS mit idealem Point \\
\hline
\end{tabular}

\subsubsection{Multi Attribute Decision Making}

Bei den Entscheidungen ohne Informationen über die Präferenzen des Entscheidungsträgers werden die Alternativen anhand ihrer extremen Werte beurteilt. Liegen die Informationen über Entscheidungskriterien (Attribute) in Form eines Anspruchsniveaus oder einer Mindestgrenze vor, dann werden nur solche Alternativen akzeptiert, die sie erreichen. Die Verfahren, bei denen nur ordinal-messbare Informationen (relative Wichtigkeit) über Entscheidungskriterien vorausgesetzt werden, gehen von einer attributweisen Rangordnung der Alternativen oder einem Gewichtvektor aus.

Die Methoden, welche kardinal messbare relative Wichtigkeit der Attribute voraussetzen, haben implizit einen kompensatorischen Charakter. Sie unterscheiden sich in der Weise der Ermittlung der besten Lösung. Die einfache additive Gewichtung ist das meist verbreitete und bekannte Verfahren. Ihre Stärke liegt darin, subjektive Einflüsse transparent $\mathrm{zu}$ machen und in das Verfahren einfließen zu lassen. Für alle Attribute wird die Höhenpräferenz in Form einer Wertfunktion und anschließend die Artenpräferenz in der Form von Gewichten bestimmt. Die Wertaggregation der Höhen- und Artenpräferenz wird additiv vorgenommen. Mit Gewichten wird der Einzelnutzen eines Attributes mit dem der übrigen Attribute vergleichbar. Die Vergabe von Gewichten ist holistisch, d.h. sie findet durch die ganzheitliche Betrachtung der Wichtigkeit eines Attributes in bezug auf sämtliche übrigen Attribute statt. Sie ist entkoppelt von der Bestimmung der Höhenpräferenz, konkrete Ausprägungen der Alternativen spielen dabei keine Rolle. 
Die hierarchische additive Gewichtung wird im Analytic Hierarchy Process (AHP) benutzt. Bei dieser Methode wird ein hierarchisches Zielsystem angewendet, das mit einem Ober- oder Erfolgsziel anfängt und weiter in Ziele, Kriterien und Unterkriterien zerlegt wird.

Der ET vergleicht je zwei Alternativen bezüglich eines Kriteriums einer Hierarchieebene und die Kriterien dieser Ebene in bezug auf ein Kriterium der übergeordneten Ebene. Eine Alternative $a_{i}$ wird mit allen anderen Alternativen $a_{j}$ bezüglich des Attributs $k$ verglichen. Der AHP geht nun davon aus, dass Paarvergleiche durch Verhältnisse ausgedrückt werden. Der Entscheidungsträger muss nun eine Auskunft geben, um wieviel eine Alternative $i$ hinsichtlich des Attributs $k$ besser ist als Alternative $j$. Um eine konsistente Matrix der Paarvergleiche zu konstruieren, die so wenig wie möglich von der empirisch gegebenen Paarvergleichsmatrix abweicht, wird der regressionsanalytische Ansatz oder die sog. Eigenwertmethode angewendet. Die Bestimmung der Gewichte verläuft analog $\mathrm{zu}$ der Wertfunktion. Entscheidend ist, dass - wie in der Nutzwertanalyse üblich - die Bestimmung der Gewichte unabhängig von der Wertfunktion erfolgt.

Die letzte Gruppe der Verfahren geht von einem paarweisen Vergleich der Alternativen aus. In LINMAP wird beispielsweise zuerst die Position der idealen Lösung gesucht, um die Alternativen nach dem Abstand zu ihr anordnen zu können. Dazu wird die Präferenz des Entscheidungsträgers in Form von geordneten Alternativenpaaren $k$ und $l$ festgelegt. Die Alternativen müssen paarweise verglichen werden. Ist die Präferenz konsistent, muss gelten, dass der Abstand der vorgezogenen Alternative $k$ von der unbekannten idealen Lösung kleiner ist als der von $l$. Die Position der idealen Lösung wird so gefunden, indem das Maß der Verletzung dieser Bedingung in der geäußerten Präferenz minimiert wird.

\subsubsection{Multi-objective decision making MODM}

Die Methoden der Multi-objective decision making werden in zwei Klassen eingeteilt (ZIMMERMANN \& GUTSCHE 1991): Methoden mit a priori Information (oder Kompromissmodelle) und interaktive Methoden mit progressiven Informationen (Tabelle 11).

Die erste Klasse fasst die Methoden zusammen, bei welchen der ET die Informationen über seine Präferenzvorstellungen vor dem Einsatz eines Verfahrens bekannt gibt (a priori Informationen). Die k-dimensionalen Zielfunktionsvektoren werden durch eine eindimensionale, reellwertige Funktion f (Kompromißfunktion) bewertet, in welcher die Präferenzvorstellungen des Entscheidungsträgers festgelegt sind. Die Voraussetzung dieser Methoden ist die numerische Lösbarkeit des Problems. Außerdem muss es unter den Optimallösungen der bewerteten Funktionen eine Lösung geben, die bezüglich des Vektormaximierungsproblems funktionaleffizient ist (Kompromisslösung). 
Tab. 11: Klassifikation von MODM - Methoden (nach Hwang \& Masud 1979).

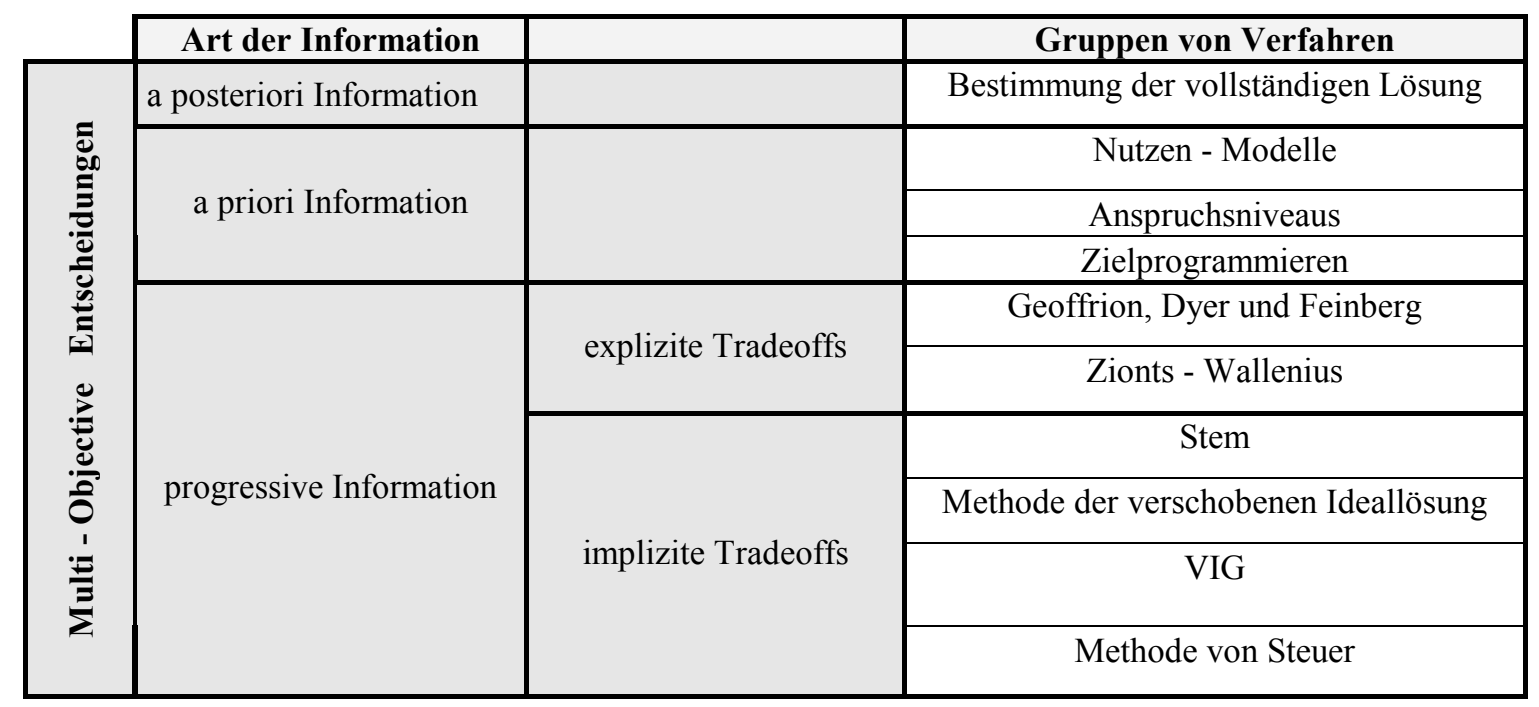

$\mathrm{Zu}$ diesen Verfahren zählen mehrere Klassen der Methoden, die an die MADM Methoden erinnern. Die Nutzenmodelle fassen sämtliche Zielfunktionskomponenten zu einer gemeinsamen Nutzenfunktion (Gesamtnutzenfunktion) U zusammen, die zu maximieren ist. Wie bei den multiattributiven Methoden stellt sich die Frage der Existenz und der Form einer solchen Funktion. Insbesondere die additive Form ist nur unter wechselseitiger Präferenzunabhängigkeit gegeben. Die Präferenzunabhängigkeit lässt sich durch eine geeignete Strukturierung des Entscheidungsproblems mindestens annäherend erreichen.

Die Verfahren mit Anspruchsniveau bezeichnen Methoden, bei welchen der Entscheidungsträger für alle Ziele bis auf eines reelle Zahlen $u$ als Mindestwerte (oder $o$ als Höchstwerte) angibt. Die Ziele des Entscheidungsträgers können so in Form eines Satisfaktionsziels angegeben werden. Ob das vorgegebene Zufriedenheitsniveau nur mit Gleichheit erreicht wird oder ob es wesentlich überschritten wird, hat keine Auswirkung auf die Präferenz.

Die Zielprogrammierung (Goal Programming) bezeichnet eine Klasse der Entscheidungsmethoden, bei welchen die optimalen, angestrebten Zielwerte vorgegeben sind. Da die gleichzeitige Erreichung aller dieser Zielwerte nicht möglich ist, müssen die Abstände von den Zielwerten präferenzorientiert bewertet und geordnet werden. Je nach der Art, wie die Zielwerte angenähert werden, unterscheidet man ein Archimedisches und ein Lexikographisches Zielprogrammieren. Im ersten Fall wird die Nähe $\mathrm{zu}$ vorgegebenen Werten durch eine Abstandsfunktion gemessen und bewertet. Im zweiten Fall sind die Zielwerte nach ihrer Wichtigkeit geordnet und ihre Erreichung wird nach ihrer Wichtigkeit in einer lexikographischen Reihenfolge angestrebt.

Bei interaktiven Verfahren wird die Unfähigkeit des Entscheidungsträgers berücksichtigt, seine Präferenzvorstellungen vollständig wiederzugeben. Der ET hat nur eine vage Vorstellung von der Zielfunktion oder von den individuellen Optima und dem Lösungsraum oder will die Entscheidung nicht nach reinem mathematischem Optimieren treffen. Die Anwendung progressiver Informationen bedeutet, dass sie erst während des Verfahrens verlangt werden. Im Mittelpunkt des Verfahrens steht die Interaktion mit dem ET. Der Entscheidungsträger macht lokale Angaben über seine Präferenzen und bestimmt damit, wie das Verfahren fortgesetzt 
werden soll. Lokal bedeutet, dass der Entscheider nicht eine gesamte Präferenzfunktion bezüglich aller Zielfunktionen angeben muss, sondern dass nur seine Präferenzen hinsichtlich einzelner Ziele oder Alternativen oder ihrer Umgebung erfragt werden. Die gefundenen Ergebnisse hängen neben der Alternativenmenge, der vektoriellen Zielfunktion und den allgemeinen Präferenzen auch davon ab, wie der interaktive Prozess abläuft. Die lokalen Informationen verkleinern den Lösungsraum oder die betrachteten Teile der vollständigen Lösung, bis eine funktional-effiziente Lösung gefunden wird.

\subsection{Entscheidungsunterstützungssysteme}

\subsubsection{Decision Support System}

Der Begriff 'Decision Support System' DSS wurde bereits zu Beginn der 70-er Jahre in den USA eingeführt (HÄNLE 1993). Als Kern der sich immer weiter entwickelnden Definition dieses Begriffes ist die Integration von Entscheidungsproblem, Computersystem und Entscheidungsträger (ET) zu sehen. In den letzten Jahren, dank fortgeschrittener Entwicklung der Informationstechnologien, ist die Domäne dieser Systeme breiter geworden und umfasst jede Art von computergestütztem System, das positiv auf eine Entscheidungsfindung wirkt. "EIS, ESS, GIS, OLAP, software agents, knowledge discovery systems and group DSS can all be lumped into the category of systems we call DSS" (POWER 1999).

Entscheidungsunterstützungssysteme unterstützen den ET in einer nicht- oder nur teilweise strukturierten Entscheidungssituation. Die semi-strukturierten Entscheidungsprobleme liegen im Bereich zwischen vollständig strukturierter und vollkommen unstrukturierter Entscheidung (vgl. SIMON zitiert nach WERNER 1992). Die strukturierten Probleme stellen keine großen Anforderungen an den Entscheidungsträger, sind programmierbar und können vom Rechner gelöst werden. Unstrukturierte Probleme müssen vom Entscheidungsträger ohne Hilfe des Rechners gelöst werden. Das DSS stellt keinen Entscheidungsautomat dar, d.h. es ist nicht in der Lage, den ET zu ersetzen. Es unterstützt ihn bei der Entscheidungsfindung. Die Entscheidungsunterstützung will helfen, rasch gute und nachvollziehbare Entscheidungen in den verschiedensten Entscheidungssituationen zu finden (CAMESELE 1998).

Ein DSS ist im Allgemeinen ein Informationssystem, das die vorhandenen problemrelevanten Informationen entscheidungsorientiert verarbeitet. DSS „.. pflegen, Entscheidungsträger in verantwortlicher Stellung in der Regel Entscheidungen vorzubereiten, meist unter erheblichem Einsatz an Ressourcen, Personal, Zeit und im Austausch mit vielen Interessierten " (HÄTTENSCHWILLER 1997). Nach JANSSEN (in SCHWAPP 1995) ist ein DSS „ein Computerprogramm, das Individuen oder Gruppen in ihrem Entscheidungsprozess hilft, das vielmehr die Urteilsfindung dieser Individuen unterstützt als ersetzt und das vielmehr die Wirksamkeit als die Rationalität eines Entscheidungsprozesses verbessert". Dabei ist DSS nicht als eine isolierte Technologie, sondern als ,eine umfassende Sichtweise des Managements, die Rolle computergestützter Systeme als Managementwerkzeuge zu verstehen “(HÄNLE 1993 S. 35). 
Ein DSS kombiniert die Vorteile des menschlichen Übersichts- und Urteilsvermögens mit der Zuverlässigkeit und Schnelligkeit von computergestützten Prozessen. Die Entscheidungsunterstützung bietet folgende Vorteile für die Entscheidungsfindung (HÄTTENSCHWILLER 1997):

- Schnelle Entscheidungen bieten einen Zeitvorteil.

- Effektivere Entscheidungen führen zu besseren Lösungen.

- Effizientere Entscheidungen sind weniger aufwendig.

- Transparentere Entscheidungen sind für Dritte besser verständlich.

- Rationale, reproduzierbare Entscheidungen werden in wiederholten Fällen unter gleichen Bedingungen gleich getroffen.

- Die Entscheidungsunterstützung ist interaktiv in allen Phasen des Entscheidungsprozesses.

Ein DSS besitzt folgende Eigenschaften (nach DENSHAM 1991, zitiert nach ENACHE 1994):

1) Design für die Lösung unstrukturierter Probleme.

2) Leistungsvolle und einfach handhabbare Oberfläche.

3) Fähigkeit zur flexiblen Kombination analytischer Modelle mit Daten.

4) Fähigkeit, mit Hilfe von Alternativen den Lösungsraum zu untersuchen.

5) Fähigkeit, verschiedene Stile der Entscheidungsunterstützung anzubieten.

6) Es erlaubt die interaktive Problemlösung mit Rückkopplung.

Tab. 12: Abgrenzung der Entscheidungsunterstützungssysteme (nach CZERANKA 1997)

\begin{tabular}{|c|c|c|}
\hline Art des Wissens, das für die & \multicolumn{2}{|c|}{ Art der Probleme } \\
\cline { 2 - 3 } Problemstellung notwendig ist & $\begin{array}{c}\text { struktuierte (oder } \\
\text { routinemäßige ) Probleme }\end{array}$ & $\begin{array}{c}\text { un - oder } \\
\text { semistruktuierte } \\
\text { Probleme }\end{array}$ \\
\hline $\begin{array}{l}\text { Quantitatives Schlußfolgern: } \\
\text { Numerische Berechnung }\end{array}$ & Datenverarbeitungssystem & $\begin{array}{c}\text { Decision - Support } \\
\text { System }\end{array}$ \\
\hline $\begin{array}{l}\text { Übergang: u.a. Entwicklung } \\
\text { heuristischer Methoden }\end{array}$ & $\begin{array}{c}\text { wissensbasiertes } \\
\text { Expertensystem }\end{array}$ & $\begin{array}{c}\text { Decision - Support } \\
\text { System }\end{array}$ \\
\hline $\begin{array}{l}\text { Qualitatives Schlußfolgern: } \\
\text { Intuition, Daumenregeln }\end{array}$ & Expertensystem & Menschlicher Experte \\
\hline
\end{tabular}

Um das Entscheidungsproblem lösen zu können, werden verschiedene Arten des Wissens benötigt (DENSHAM 1991, CZERANKA 1997). Das allgemeine Wissen wird benötigt, um die Problemumgebung zu erkennen. Das prozedurale Wissen entwickelt den Lösungsprozess und bestimmt die Parameter oder Werte der Modelle. Das strukturelle Wissen bezieht sich auf die Datenstruktur sowie die Umsetzung ausgewählter Modelle.

An der Entwicklung, Wartung und Anwendung eines DSS sind mehrere Personengruppen beteiligt. Der Entscheidungsträger ist der Nutzer von DSS, seine Entscheidungsprobleme werden mit Hilfe eines DSS gelöst. Der Entscheidungsberater ist der Vermittler der Entscheidungsfindung, oder der Benutzer von DSS. Die Methodiker pflegen die Modellbank, während die Informatiker für die Implementierung und Wartung von DSS sorgen.

Ein DSS besteht nach dem DDM-Paradigma aus folgenden Komponenten

- Komponente zur Ablauf- und Dialogsteuerung (Dialoge)

- Datenkomponente (Daten)

- Modell- oder Methodenkomponente (Modelle) 
Diese Subsysteme stehen in einer starken wechselseitigen Beziehung zueinander. Der Benutzer (Anwender) kommuniziert mit dem DSS über das Dialogmanagementsystem. Dieses steht in Verbindung mit beiden übrigen Komponenten.

Unter Dialogkomponente versteht man die Steuerung der Ein- und Ausgabe der Entscheidungsfindung. Die wichtigsten Komponenten dieses Subsystems sind der ET, das Terminal oder der PC und das Dialogmanagementsystem. Das Datensubsystem besteht aus einer Datenbank und einem Datenbankmanagementsystem. In die operative Datenbank (Front-endDatenbank) werden Daten aus einer zentralen Datenbank (Back-end-Datenbank) über ein Datenextraktions- oder -konvertierungssystem überführt (HäNLE 1993). Das Datenbankmanagementsystem enthält Funktionen, mit denen die Daten erfasst, abgerufen, modifiziert, selektiert und gelöscht werden können. Das Modellsubsystem unterstützt die Entscheidungsfindung mittels Modellen. Die Modelle stellen konkrete Entscheidungssuchen dar, welche aus den Methoden zusammengesetzt werden. Ein Modellbankmanagementsystem unterstützt die Modellbildung und -verwaltung. Die Verfügbarkeit von Entscheidungshilfen in Form von Modellen und Methoden der Entscheidungsfindung ist die wichtigste Eigenschaft von DSS. Es geht dabei in einer groben Gliederung um folgende Modelle:

- Modellierung der Entscheidungsalternativen

- Modellierung der Konsequenzen der Alternativen

- Modellierung der Bewertungskriterien

- Modellierung der Präferenzen des ET

- Synthese der Entscheidungsvorschläge

Die Entscheidungsunterstützungsmethoden übernehmen verschiedene Funktionen (CZERANKA 1997) im Entscheidungsprozeß. Die fachwissenschaftlichen Methoden unterstützen die Auswahl der bei einer bestimmten Fragestellung zu berücksichtigenden Kriterien. Aggregationsmethoden ermöglichen eine Datenreduktion bei Bewahrung eines möglichst hohen Informationsgehaltes. Standardisierungs- und Normalisierungsmethoden vergleichen die Kriterien miteinander. Bewertungsmethoden berechnen die zielbezogenen Wertigkeiten aus den Kriterienausprägungen. Evaluierungsmethoden vergleichen diese Wertigkeiten hinsichtlich der Ziele und ermitteln die in Frage kommenden Alternativen. Die Entscheidungsmethoden unterstützen die Auswahl der optimalen Alternative oder die Erstellung einer Rangordnung.

\subsubsection{GDSS (Group decision support systems)}

Ein DSS geht davon aus, dass der ET isoliert und einzeln seine Entscheidungen trifft. Im Gegensatz dazu unterstützen die GDSS eine ganze Gruppe von Entscheidungsträgern, eine gemeinsame Entscheidung zu treffen. Die Gruppenentscheidungsunterstützung basiert auf Kommunikation und sozial-psychologischen Aspekten der Gruppen. Ein GDSS kombiniert Computer, Kommunikation und Decision Support Technologien in einer Einheit. Ein GDSS erweitert ein herkömmliches DSS um die Kommunikation, ihre Planung und Leitung, und um eine strukturierte Entscheidungsanalyse.

Während ein GDSS eine Gruppe von ET in einem Entscheidungsprozeß unterstützt, werden Group Support Systeme für die gegenseitige Abstimmung der einzeln getroffenen Entscheidungen verwendet. Im Unterschied zu DSS muss die Dialogkomponente also einen Informationsaustausch unterstützen (Multiuser-Interface). 
Mit dem Aufkommen der globalen Netzwerke entstand eine vorher unbekannte Gruppenorganisationsform, die sog. virtuelle Gruppe. Der virtuelle Gruppenraum entsteht durch den Einsatz von GroupWare (integrierte Netzwerkanwendungen). Ihre Realisierung integriert die theoretischen Grundlagen, die im Rahmen von Computer Supported Cooperative Work (CSCW) spezifiziert werden. CSCW bezeichnet die theoretischen Grundlagen bzw. die Methodologien für Gruppenarbeit und deren Computerunterstützung.

Die GDSS eröffnen von ihrem Potential her weit mehr als eine Summe der Funktionalitäten von DSS und GroupWare. "Beispielsweise ist eine Wiederverwendbarkeit und Austauschbarkeit von umfassenden Anwendungsobjekten der Entscheidungsunterstützung (wie etwa Szenarien) zwischen kooperierenden Partnern ein neues Element, ein Exchangemanager eine diesbezügliche neue Funktionalität und die integrierte Arbeitsteilung zwischen verschiedenen Rollenträgern eine diesbezüglich neue Möglichkeit der Entscheidungsunterstützung" (HÄTTENSCHWILLER 1998, S.18).

Je nach dem Grad der Intensität der Zusammenarbeit innerhalb einer Gruppe kann zwischen Kommunikation, Koordination und Kooperation unterschieden werden. Bei der Zusammenarbeit können die Gruppenmitglieder am gleichen oder an verschiedenen Orten sein (räumliche Verteilung). Die zeitliche Verteilung von Gruppen unterscheidet eine synchrone Gruppenarbeit, wenn die Mitglieder in Realzeit kommunizieren, und asynchrone Gruppenarbeit, wenn die Kommunikation nicht in Realzeit erfolgt.

Die mit Hilfe der Computertechnologie unterstützten Aktivitäten einer Gruppe bestehen nach HÄNLE (1993) aus:

- Verknüpfung der verteilten Datenbeständen.

- Anwendung der gruppenbezogenen Techniken und Methoden.

- Informationsaustausch in vernetzter Umgebung.

Es werden mehrere Entwicklungsstufen von GDSS unterschieden, je nachdem, wie die Unterstützungsfunktion des Systems gestaltet ist:

Stufe 1: Kommunikationsunterstützung

Auf dieser Stufe wird der Informationsaustausch zwischen den Gruppenmitgliedern erleichtert (durch eine geeignete Strukturierung vereinfacht) und die Kommunikationsbarrieren werden abgebaut (vgl. WERNER 1992). Als technische Mittel zählen dazu z.B. e-mail, Großbildschirm, LAN usw.

\section{Stufe 2: DSS Funktionen}

GDSS dieser Art besitzen auch Techniken zur Modellierung und Komplexitätsreduzierung von Entscheidungsproblemen, Ausführung und Berechnung von Modellen, Interaktions- und Darstellungsoptionen etc. Auch Gruppentechniken wie Brainstorming, Delphi-Methode usw. werden auf dieser Stufe unterstützt.

Stufe 3: Systemsteuerung

Auf dieser Stufe werden die Kommunikations- und Prozessstrukturen durch das System selbst bestimmt (vgl. WERNER 1992). Die GDSS werden durch den Einsatz von 
Kommunikationsmustern und Expertenwissen charakterisiert (HÄNLE 1993). Das GDSS übernimmt somit die Aufgabe eines aktiven Mediators.

Werden verschiedene Gruppen zu einer übergeordneten Gruppe zusammengefasst, so entsteht eine Organisation als übergeordnete Struktur von Gruppen mit hierarchisch aufgebauten Zielen. In einer Organisation besteht zwischen den Gruppen in der horizontalen Richtung ein Wettbewerb, in der vertikalen Richtung eine Kooperation (vgl. HÄTTENSCHWILLER 1998). Eine Entscheidungsunterstützung in der Organisation erhält so eine weitere Dimension der kooperativen Zusammenarbeit, und die Systeme der Entscheidungsunterstützung werden danach auch als Organisational Decision Support Systems (ODSS) bezeichnet. 


\section{Räumliche Entscheidungen}

"What do $80 \%$ of all business and policy problems involved in creating livable communities have in common? Location, location, location. Whether responding to emergencies, finding a suitable locatio to store hazardous materials, determining the quality and extent of habitat, or setting quidelines for extracting natural resources, geospatial data and relationships are key to decision making" (ANONYMUS 1999F).

\subsection{Einleitung}

\subsubsection{Einführung und Motivation}

Räumlichkeit ist ein grundlegendes Strukturierungskonzept, 80\% der Informationen haben einen räumlichen Bezug. Mit räumlichen Entscheidungen ist der Mensch täglich konfrontiert, seine Bewegung erfolgt aufgrund einer Vielzahl unbewusster oder bewusster Entscheidungen. Diese werden mit Hilfe seiner kognitiven Fähigkeiten im Prozess mentaler Informationsverarbeitung getroffen, durch den die wichtigsten Aspekte der Realität abstrahiert, abgebildet, gespeichert und verknüpft werden.

Tab. 13: Vergleich von mentalen und formalen Modellen (in Anlehnung an KOLLARITS 1999).

\begin{tabular}{|c|c|c|c|}
\hline \multicolumn{2}{|c|}{ Aspekte } & Kognitive Modelle & Formale Modelle \\
\hline \multicolumn{2}{|c|}{ Eigenschaften } & $\begin{array}{l}\text { Subjektivität } \\
\text { Gebundenheit an die Umwelt } \\
\text { Erlernen } \\
\text { Unvollständigkeit }\end{array}$ & $\begin{array}{l}\text { Nachvollzierbarkeit } \\
\text { Transparenz } \\
\text { Wiederholbarkeit } \\
\text { Vollständigkeit / Konsistenz } \\
\text { Implementierbarkeit }\end{array}$ \\
\hline \multicolumn{2}{|c|}{ Charakteristik } & intuitiv-empirisch & logisch-deduktiv \\
\hline \multicolumn{2}{|l|}{ Ziel } & Orientierung & Abbildung für bestimmten Zweck \\
\hline \multirow[t]{2}{*}{ Struktur } & topologisch & Handlungsgrundlage & unvollständig \\
\hline & metrisch & relativ, verzerrt & absolut \\
\hline \multirow[t]{2}{*}{ Inhalt } & Objekte & gewichtet, schwer formalisierbar & ungewichtet, diskret \\
\hline & Vollständigkeit & Auswahl & vollständig für Objektkategorie \\
\hline
\end{tabular}

Die Entscheidungsträger treffen ihre Entscheidungen auf Basis ihrer Einschätzung der Realität, der Struktur und Zusammensetzung des beplanten Systems (vgl. REINBERG 1998). Bei leicht überschaubaren Problemen mit kleiner Komplexität sind Menschen in der Lage, schnell zu entscheiden und $\mathrm{zu}$ handeln. Dabei können Intuition, Erfahrung und subjektive Urteile angewendet werden. Menschen haben oft keine Schwierigkeiten mit der Verarbeitung unscharfer Informationen und der Anwendung unvollständig strukturierten Wissens. Die zu berücksichtigende Komplexität der Zusammenhänge und Einflüsse in dem realen System, sowie die immer komplexer werdenden Ansprüche und anzustrebenden Ziele führen dazu, dass die menschliche Kognitionsfähigkeit überfordert wird (Tabelle 13). Nach DöRNER (1992, zitiert nach REINBERG 1998) sind für die Unzulänglichkeit menschlichen Denkens vor allem folgende kognitive Eigenschaften verantwortlich:

- Langsamkeit des Denkens

- Unfähigkeit der effizienten Erfassung großer Informationsvolumina 
- Kompetenzbewahrungstendenz

- geringe ,Zuflusskapazität' des Gedächtnisses (Überwertigkeit des aktuellen Motivs).

Die Kognitionswissenschaft untersucht Situationen, in welchen rein kognitionsbasierte Entscheidungen an ihre Grenzen stoßen. Die mangelhaften kognitiven Fähigkeiten des Menschen werden in komplexen Entscheidungssituationen durch formale Modelle unterstützt (Tabelle 13). Ihre Aufgabe ist die Entlastung von Menschen durch geeignete Strukturierung und Aufzeigen der Lösungsmöglichkeiten von Entscheidungsproblemen.

Räumliche Entscheidungsfindung (spatial decision analysis, THILL 1999) behandelt Handlungssituationen, in welchen die Räumlichkeit für die Beurteilung der Alternativen von Bedeutung ist. Sie umfasst die Anwendung von Entscheidungsmethoden und Modellen in räumlicher, insbesondere geographischer Domäne. Die wichtigste Aufgabe räumlicher Entscheidungsfindung ist es, mit Hilfe formaler und implementierbarer Methoden dem Entscheidungsträger die Formulierung seiner Kriterien und der Abbildung seiner Präferenzen über Alternativen zu ermöglichen. Die mentalen Modelle werden dabei in formale überführt, auf Konsistenz überprüft, verarbeitet um schließlich durch die Präferenzabbildung eine Lösungsfindung zu ermöglichen. Die räumlichen Entscheidungsunterstützungssysteme (Spatial Decision Support Systems SDSS) sind Werkzeuge, welche den Entscheidungsträger durch Generierung oder Bewertung von räumlichen Alternativen oder durch Handhabung von entscheidungsrelevanten räumlichen Objekten und Beziehungen unterstützen.

Räumliche Entscheidungsfindung stellt einen Überlappungsbereich der GIScience und der Entscheidungstheorie dar (Abbildung 40). GIScience erforscht räumliche Konzepte, die mit Methoden der Entscheidungstheorie zur Lösung räumlicher Entscheidungsprobleme angewendet werden.

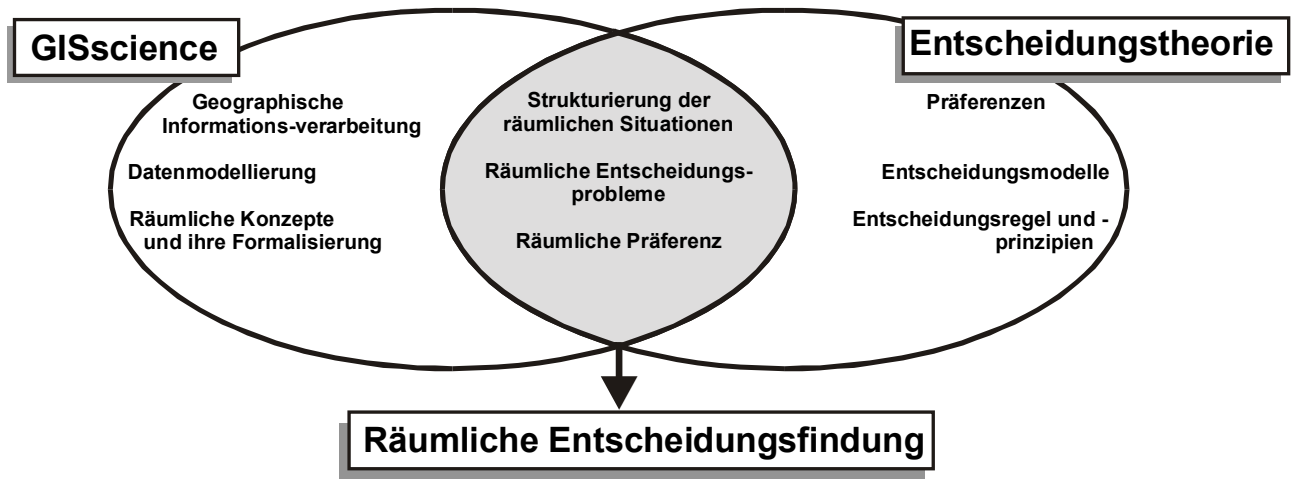

Abb. 40: Beziehungen der räumlichen Entscheidungsfindung.

Die Aufgaben räumlicher Entscheidungsfindung (Tabelle 14) reichen von der Untersuchung der räumlichen Aspekte angestrebter Ziele eines Entscheidungsträgers bis zur Konstruktion räumlicher Entscheidungsunterstützungssysteme. Während als indirektes Produkt der Entscheidungstheorie das Entscheidungsunterstützungssystem und der GIScience das Geographische Informationssystem (GIS) angesehen werden kann, werden bei der Konstruktion von SDSS die Ergebnisse beider Wissenschaften im gleichen Masse angewendet. 
Tab. 14: Aufgaben der räumlichen Entscheidungsfindung.

\begin{tabular}{c|} 
Identifikation \\
Strukturieren \\
Abbilden \\
Anpassen \\
Konstruieren
\end{tabular}

\author{
der Räumlichkeit in Zielen \\ räumlicher Entscheidungsprobleme \\ räumlicher Präferenz \\ räumlicher Entscheidungsmodelle \\ räumlicher Entscheidungsunterstützungssysteme
}

\subsubsection{Anwendungsübersicht}

Räumliche Entscheidungsfindung findet Anwendung in allen Bereichen, in welchen zwischen alternativen Handlungen mit unterschiedlichen räumlichen Konsequenzen entschieden werden soll. Fragestellungen wie Ausscheidung potentieller Schutzgebiete, Entscheidungen über Flächennutzungen, flächenbezogene Bewertung, Bestimmung des Absatzgebietes oder Standortsuche und -relokation können mit Hilfe räumlicher Entscheidungsfindung gelöst werden.

Der an räumliche Entscheidungen traditionell reiche Bereich ist die Raumplanung. Sie befasst sich mit der Koordination von Maßnahmen, welche auf die Standortqualität wirken und wirken sollen und welche die Verteilung der Standortqualität über die Bevölkerung verändern (BöCKEMANN 1993, S.4). Standorte konstituieren Siedlungsstrukturen, diese sind Grundlage für soziale Prozesse, Rahmen für marktlichen Austausch und Gegenstand politischer Steuerung (VOIGT 1999). Der Gegenstand der Raumplanung ist das räumliche Nebeneinander des gesellschaftlichen Zusammenlebens. Raumplanung ist der Versuch, das Nebeneinander konkurrierender und komplementärer Raumnutzungen rational zu gestalten (FRANK G. 1997).

Wegen der konkurrierenden Nutzungsabsichten der Umweltressourcen findet räumliche Entscheidungsfindung immer breiteren Einsatz auch im Naturschutz. Naturschutz versteht sich als Gesamtheit der Maßnahmen zur Erhaltung und Förderung der natürlichen Lebensgrundlagen, aller Lebewesen, insbesondere von Pflanzen und Tieren wildlebender Arten und ihrer Lebensgemeinschaften sowie zur Sicherung von Landschaften und Landschaftsteilen in ihrer Vielfalt und Eigenart (VOGEL \& BLASCHKE 1996). Ihre Aufgaben, die in Verbindung mit räumlichen Entscheidungen in vielfältigen Beziehungen stehen, umfassen beispielsweise Artenschutz, Biotopschutz, Ausweisen von Reservaten, Schutz der abiotischen Naturgüter Wasser, Boden und Luft, Steuerung der Landnutzung etc.

Als weitere Anwendungsbereiche räumlicher Entscheidungsfindung können Geomarketing und teilschlagspezifische Landbewirtschaftung (Precision Farming) erwähnt werden:

Geomarketing (business geographics) ist eine interdisziplinäre Fachrichtung, welche die Wirtschaftsgeographie mit der GIS Technologie verbindet. Es versucht, durch einen zielorientierten Einsatz der GIS Technologie und der geographischen Datenanalyse und Visualisierung eine höhere Qualität der marktrelevanten Maßnahme von Entscheidungsträgern effektiver und effizienter $\mathrm{zu}$ erzielen. GIS wird in diesem Umfeld als strategisches Managementinstrument verstanden. Eine besondere Rolle als Grundlage für Geomarketing und Vertrieb sowie zur Optimierung der Geschäftsprozesse kommt den Geodaten (Dataware) zu. Räumlich referenzierte, betriebsinterne Daten (Lieferanten und Kunden) werden mit betriebsexternen Marktdaten verbunden (soziodemographische Daten und Daten der administrativen und statistischen Einheiten), um unternehmerische Entscheidungen vorzubereiten und zu unterstützen. Ein zielorientierter Einsatz der Geodaten im Rahmen des Geomarketing 
ermöglicht verbesserte Marktsegmentierung und Gebietstypisierung anhand von Kundenmerkmalen. Standort- und Kundenpotentialanalyse, Wahl der Filialstandorte, regionale Anpassung der Produktpalette, Wahl der Absatzgebiete, Steuerung der Distributionsplanung, Werbemitteleinsatzplanung, Marktpotentialuntersuchungen, Konkurrenz- und Wettbewerbsanalysen - Standortwahl und Standortevaluierung zählen zu den Anwendungsbeispielen des Geomarketings.

Precision farming oder Präzisionspflanzenbau (teilschlagspezifische Landbewirtschaftung) ist räumlich angepasste Steuerung komplexer Produktions- und Bearbeitungsgänge in der Landwirtschaft mit Hilfe der geographischen Informationssysteme und des Globalen Positioning Systems (GPS). Das bedeutet, dass Aussaat, Düngung und Pflanzenschutz gemäß den Unterschieden in Boden und Pflanzenbestand bemessen werden. Teilschlagspezifische Bewirtschaftung ermöglicht die zeitnahe Regelung von Pflanzbeständen nach Standortpotential und aktuellem Versorgungszustand der Pflanzen mit bodengebundenen Wachstumsfaktoren. Ihr Ziel ist es, durch eine ökologische und ökonomische Optimierung des Betriebes eine betriebswirtschaftlich gerechtfertigte und nachhaltige Landnutzung zu ermöglichen. Dabei spielt die räumliche Entscheidungsfindung eine wichtige Rolle bei der Beurteilung lokaler bis regionaler Nutzungskonflikte bezüglich der Landnutzung und des Boden- und Gewässerschutzes und bei Behandlungsentscheidungen einzelner Teilschläge. Man spricht von einem Paradigmenwechsel in der Landwirtschaft, der alle Bereiche der modernen Landwirtschaft umfasst.

\subsubsection{Stand der Forschung und Literaturübersicht}

Für die Entwicklung der räumlichen Entscheidungsfindung waren u.a. die Aktivitäten der NCGIA (National Centre for Geographic Information Analysis) von großer Wichtigkeit. Die Bedeutung der räumlichen Entscheidungsunterstützungssysteme in Kombination mit der Nutzung der GI und GIS war bereits in der Initiative 6 [Spatial Decision Support Systems 1990-1993] und später in der Initiative 17 [Collaborative Spatial Decision Making 1995] erkannt. CIESIN (Center for International Earth Science Information Network) hat während eines dreijährigen Forschungsprojekts ein räumliches Entscheidungsunterstützungssystem [Active Response Geographic Information System] entwickelt, dessen Aufgabe die Unterstützung einer kollaborativen Entscheidungsfindung ist.

In Europa befasste sich das im Rahmen der „Telematics Programme“ der Europäischen Kommission finanzierte Projekt GeoMed mit den Möglichkeiten, die verteilten Geoinformationen und GIS für den Prozess der Entscheidungsfindung zugänglich zu machen. Das assoziierte Projekt ZENO beschäftigte sich mit der Entwicklung eines Mediationssystems, das die Problemformulierung, Strukturierung und anschließende Argumentation im Rahmen eines Mediationsentscheidungsverfahrens organisieren sollte.

Im Prozess der Festlegung von nationalen Forschungsprioritäten für die Jahre 1996-2005 hat UCGIS (University Consorcium for Geographic Information Science) seine Mitglieder aufgefordert, bis zu 5 Themenvorschläge einzureichen. Im Bereich GIS in Decision-Making wurden folgende Projekte eingereicht:

- GIS und Entscheidungstechniken

- Institutionale Barrieren für Nutzung von GIS in Entscheidungsfindung 
- Forschung an Verknüpfung zwischen GIS Forschung und Business/RegierungsEntscheidungen

- Decision support systems als integrierende Plattform für effektives und effizientes Management der Naturressourcen

Das erste Projekt bezieht sich auf die methodische Seite der räumlichen Entscheidungsfindung. Es umfasst die Überprüfung der vorhandenen und die Entwicklung neuer Entscheidungsmethoden für die Anwendung in der räumlichen Domäne. Explizit sollen die unscharfen Entscheidungsmethoden und multimedialen Repräsentationen in der Entscheidungsfindung mittels GIS untersucht werden. Auch die multikriterielle Integration und Präferenzabhängigkeit der Kriterien wurde von dem Vorschlag direkt angesprochen.

Das zweite Projekt beabsichtigte die Untersuchung mangelhafter Verbreitung (Penetration) der Methoden räumlicher Entscheidungsfindung in den Organisationen. Es sollten die Fragen beantwortet werden, warum GIS nicht zur Entscheidungsfindung herangezogen wird, was sich an GIS ändern soll, um den Bedürfnissen der Entscheidungsträger besser Rechnung zu tragen und den Grund für die mangelhafte Akzeptanz (Bewusstsein) von GIS als eines entscheidungsunterstützenden Werkzeuges zu ermitteln.

Die diesbezüglichen Anwendungsgebiete und Grundlagenforschungen stammen aus unterschiedlichen Bereichen. Im deutschsprachigen Raum beschäftigte sich MANDL (1994, 1995, 1998) mit theoretischen Aspekten räumlicher Entscheidungsunterstützung in GIS. SCHMIDT (1996, 1998) untersuchte unscharfe räumliche Entscheidungsprozesse und Konfliktanalyse in raumbezogenen Planungs- und Entscheidungsprozessen, CZERANKA $(1996,1997)$ befasste sich mit räumlichen Entscheidungsunterstützungssystemen in Naturschutz und Landschaftspflege, REINBERCK (1998) befasste sich mit der Simulation planerischer Entscheidungsfindung mit Hilfe kalkulatorischer Bewertungsmodelle. Landschaftsökologische Bewertung mit anschließender multikriterieller Optimierung werden von GrABAUM \& MEYER (1997) vorgestellt. Auch die Arbeiten von weiteren Autoren [SCHWAP (1994, 1995, 1996), BOGNER (1999), SCHWARZV.RAUMER $(1996,1997)$, LÜTHY (1998)] sind in diesem Kontext von Bedeutung.

Im englischsprachigen Raum sind die Arbeiten von EASTMAN (1999), MALCEWSKI (1996, 1997, 1998, 1999A, 1999B), JANKOWSKI (1995, 1999A, 1999B), DENSHAM (1991, 1994), CARVER (1996A, 1996B, 1999), ENACHE (1994), KEENAN (1997), NYERGES (1996), YuANG (1997) für die räumliche Entscheidungsfindung von Bedeutung. GIDMA (International Research Group on Geographic Information and Multicriteria Decision Analysis) wurde als eine Forschungsgruppe mit dauerhaften Forschungsaktivitäten im Bereich der Geoinformation und der multikriteriellen Entscheidungsfindung auf internationaler Ebene ins Leben gerufen.

Neben den GIScience oder GISsystem spezifischen Zeitschriften wird das Thema der räumlichen Entscheidungsfindung von der Zeitschrift der GIDMA Gesellschaft, Journal of Geographic Information and Decision Analysis (JGIDA), behandelt. 
Tab. 15: Beispiele der Entscheidungssituationen, bei welchen die Räumlichkeit der Alternativen von besonderer Bedeutung ist.

\begin{tabular}{c|l} 
Standortsuche & $\begin{array}{l}\text { Positionierung einer Feuerwehrüberwachungsstelle (MALCZEWSKI et al. 1997); } \\
\text { (KWAN M.P. 1998) } \\
\text { Positionierung eines Einzelhandels (KWAN M.P. 1998) } \\
\text { (Müll)Deponiestandortsuche (BLASCHKE 1997) }\end{array}$ \\
\hline Zonierung & Forstliches Abgrenzungssystem (CAMERON \& ABEL 1996) \\
\hline Eignung, & $\begin{array}{l}\text { Landnutzung (KWAN M.P. 1998) } \\
\text { Bewertung von Freiräumen als potentielles Wohnbauland } \\
\text { (ScHWARZ-V.RAUMER 1996) } \\
\text { Wohn- und. Betriebsstandortekonzept (SCHWAPP 1994, 1995) } \\
\text { Baueignung (WOLF 1999) } \\
\text { Neuabgrenzung der landwirtschaftlichen Vorrangzonen } \\
\text { Wohnstandortqualität (SCHWARZ v. RAUMER 1997) } \\
\text { Habitatmodellierung (BLASCHKE 1997) } \\
\text { Baulandbewertung (REINBERG \& BRÖTHALER 1997) }\end{array}$
\end{tabular}

Alle diese Aktivitäten und Veröffentlichungen belegen das wachsende Interesse an räumlicher Entscheidungsfindung.

\subsection{Räumliche Entscheidungsprobleme}

Ein räumliches Entscheidungsproblem ist ein Entscheidungsproblem (vgl. Kapitel 6.1) mit räumlichen Strukturmerkmalen, die als zielrelevante Konsequenzen der Alternativen zum Ausdruck kommen. In Abbildung 41 wird ein allgemeines Entscheidungsproblem um entscheidungsrelevante Räumlichkeit erweitert.

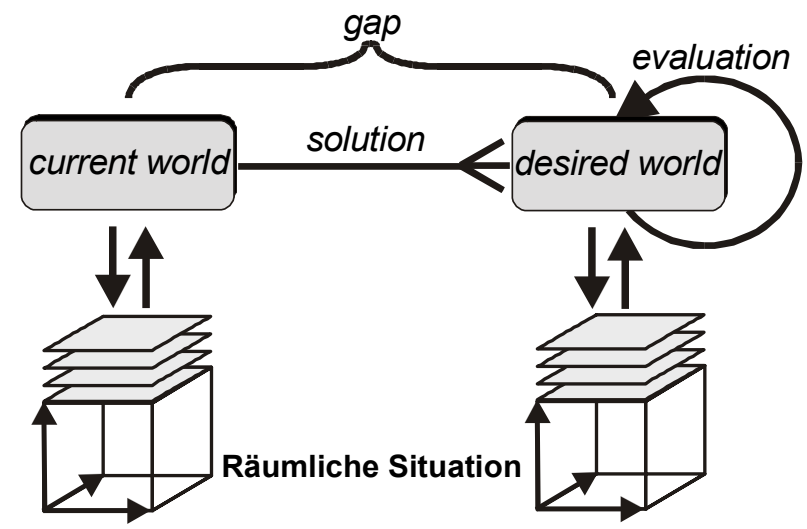

Abb. 41: Schematische Erweiterung eines Entscheidungsproblems um die Räumlichkeit.

Um ein räumliches Entscheidungsproblem lösen zu können, muss die Entscheidungssituation modelliert werden, müssen die entscheidungsrelevanten Aspekte bewertet, Präferenzen des Entscheidungsträgers abgebildet und aggregiert, und schließlich die Alternativen angeordnet werden. Im Falle räumlicher Entscheidungsprobleme geht es bei allen diesen Schritten um räumliche Konzepte (Abbildung 42). 


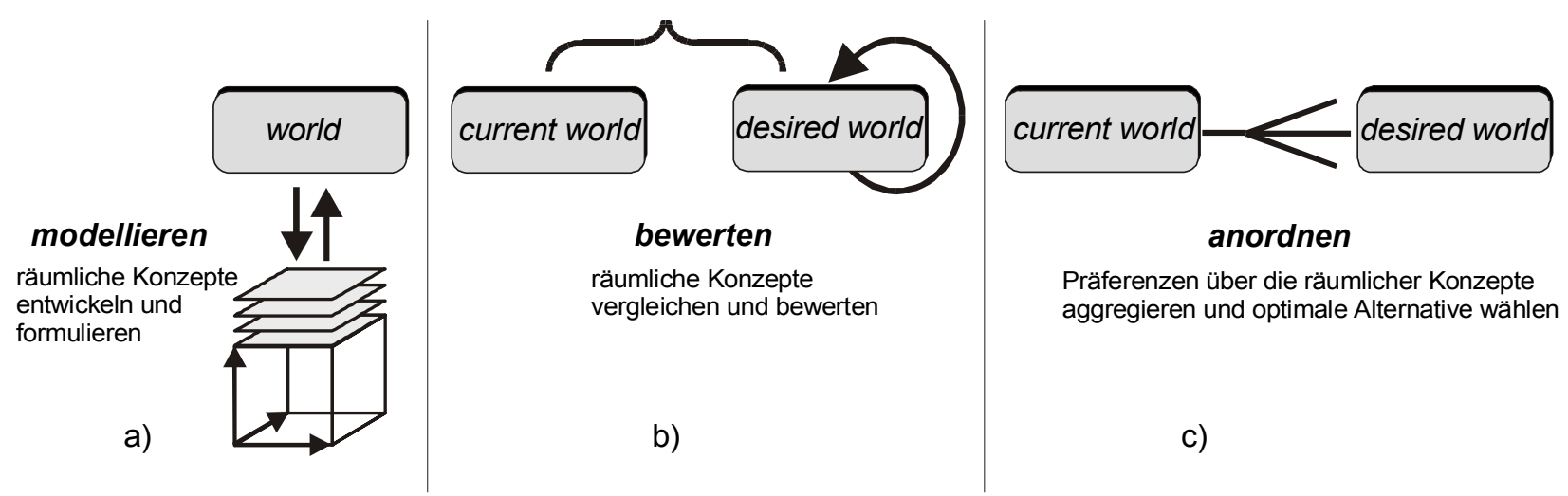

Abb. 42: Prozess der räumlichen Entscheidungsfindung.

Modellieren ist ein Prozess des Abstrahierens, während dessen die relevanten Konzepte aus der Realität erkannt und benannt werden, und ein Prozess des Strukturierens, das die abstrahierten Konzepte in Beziehungen zueinander stellt, so dass ein Modell entsteht. Bei der Bewertung müssen räumliche Konzepte miteinander verglichen werden. Dabei spielt räumliche Ähnlichkeit (vgl. Kapitel 5.2) eine wichtige Rolle. Der Unterschied eines aktuellen und eines idealen (der Zielsetzung am besten entsprechendes) Zustandes kann nur mit dem Konzept der räumlichen Ähnlichkeit erfasst werden. Oft enthält die Bewertung einen Vergleich zwischen mehreren Zuständen (vorherige und geplante), so dass sie selbst einen Entscheidungsaspekt besitzt (Verträglichkeits- bzw. Konfliktanalyse). Anordnen bedeutet die Wichtigkeit der Konzepte miteinander abzuwägen. Aggregieren erlaubt eine Anordnung solcher Modellelemente, die wegen ihrer Komplexität bezüglich mehrerer Eigenschaften (Dimensionen) bewertet werden.

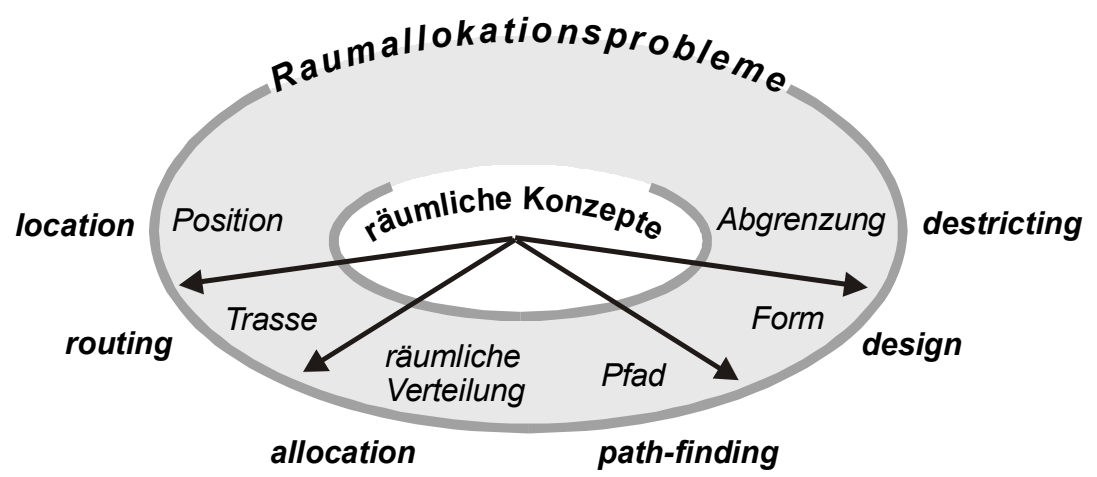

Abb. 43: Räumliche Entscheidungsprobleme und zugrundeliegende Konzepte (in Anlehnung an CZERANKA $(1997,1998)$, LAURINI \& THOMPSON (1992)).

Räumliche Entscheidungsprobleme lassen sich nach ihren Eigenschaften und Aspekten in Problembereiche einteilen (vgl. CZERANKA (1997, 1998), LAURINI \& THOMPSON 1992):

- Standortfindung (location): Finden eines optimalen Ortes oder Standortes

- Standort- oder Einzugsbereichsanalyse (allocation) Zuweisung einer optimalen Menge vorhandener räumlicher Objekte zu einem optimalen Ort im Raum

- Pfadsuche (route-finding) und Trassenlegung (path-finding): Finden einer optimalen Verbindung von zwei oder mehreren räumlichen Objekten.

- Zonierung (re-districting): Abgrenzung räumlicher Einheiten

- Layout (Design): Zuordnung von Nutzungen oder Funktionen zu Flächen nach bestimmten Gesichtspunkten. 
Die Standortsfindung (location modeling) ist eine zielgerichtete Suche nach einer Position für eine Aktivität (Einrichtung) wie Verkaufsstelle, Feuerüberwachungseinrichtung etc. Eine Übersicht der Standortsfindungsprobleme kann in CHURCH (1999) gefunden werden. Die Einzugsbereichsanalyse (allocation) ordnet oft in Anschluss an die Standortfindung (location/allocation Problem) einer mit räumlicher Position eindeutig festgelegten Aktivität einen räumlichen Bereich potentieller Inanspruchnahme der Aktivität zu.

Von ANONYMus (1999G) untersuchte Landnutzungsentscheidungsprobleme werden wie folgt gegliedert: (i) Landallocation Entscheidungen beabsichtigen eine Einteilung der Landschaft in mehrere Teile und schreiben die Behandlung oder Nutzung einzelner Teile vor. Bei (ii) Umweltentscheidungen wird ebenfalls die Landschaft in mehrere Teile zerlegt und anschließend müssen die environmentalen Fragestellungen entschieden werden, die mit dieser Zerlegung verbunden sind (z.B. Habitateignung, mitigation strategy). Risikomanagemententscheidungen identifizieren das Risiko in der Landschaft und entwickeln Szenarien um dieses Risiko zu beseitigen oder zu vermindern (z.B. Feuerüberwachung).

Räumliche Entscheidungssituationen sind durch die große Anzahl der Alternativen mit räumlichen Ausprägungen der Kriterien gekennzeichnet. Die angestrebten Ziele stehen in der Regel in Konflikt zueinander und eine Lösung ist von mehreren Entscheidungsträgern mit unterschiedlichen Präferenzen bezüglich der relativen Wichtigkeit der Alternativen und Kriterien zu wählen (vgl. MALCZEWSKI 1998). Die Kriterienausprägungen sind oft qualitativer Natur und unterliegen zeitlicher Veränderung. Die räumlichen Entscheidungsprobleme weisen einen hohen Komplexitätsgrad und sehr unterschiedlich strukturierte Problemstellungen auf (vgl. REINBERG 1998, LEUNG 1997). Die Lösung solcher Probleme verlangt ein großes Informationsvolumen und das Einfließen von Wissen und Werturteilen. Zur Lösung stehen jedoch meistens nur unvollständige Informationen zur Verfügung. Dies trägt dazu bei, dass die räumlichen Entscheidungssituationen extrem komplex und zumindest teilweise intransparent sind.

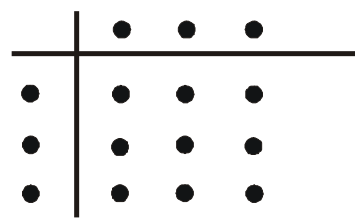

a)

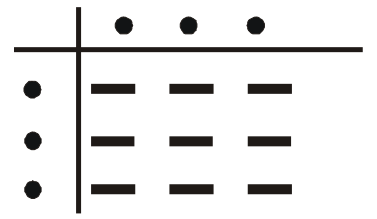

b)

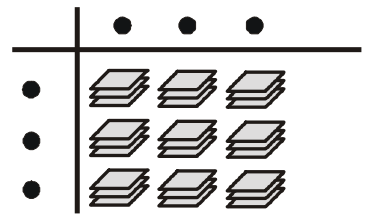

c)

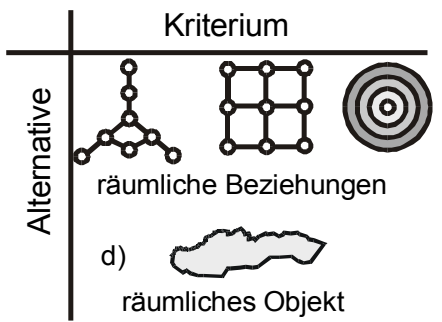

Abb. 44: Dimension der Entscheidungskriterien (in Anlehnung an VAN HERWIJNEN \& RIETVELD 1999): a) 0-dimensionales Entscheidungsproblem; b) 1-dimensionales Problem; c) 2-dimensionales Problem; d) Ausprägungen der räumlichen Entscheidungskriterien.

Räumliche Entscheidungsprobleme unterscheiden sich von a-räumlichen vor allem dadurch, dass die Entscheidungskriterien räumliche Attributsausprägungen enthalten: Entweder als Kriterienwerte oder als Strukturierungsmerkmale. Nach VAN HERWIJNEN \& RIETVELD (1999) ist die Dimension eines räumlichen Entscheidungsproblems gleich der größten Dimension ihrer Kriterien. In Abbildung 44 sind Entscheidungskriterien unterschiedlicher Dimension dargestellt.

Ein räumliches Konzept mit der Dimension 1 und 2 kann in Konzepte der anderen oder der gleichen Dimension transformiert werden: Beispielsweise kann die Entfernung zweier Orte ins 
0D Konzept (Zeit- oder Finanzkosten) oder ins 2D Konzept (Akzeptanzflächen der Distanzbeziehungen) transformiert werden.

\subsection{Modellierung räumlicher Entscheidungssituation}

Eine räumliche Entscheidungssituation setzt sich aus der räumlichen Situation, welche den räumlichen Bezug entscheidungsrelevanter Elemente beschreibt, und den Komponenten einer Entscheidungssituation (Alternativen, Kriterien) zusammen.

\subsubsection{Räumliche Situation}

Eine räumliche Situation besteht aus räumlich gleich referenzierbaren Geoobjekten, ihren Beziehungen sowie ihren Ereignissen. Die Elemente einer räumlichen Situation, die räumlichen Objekte oder Phänomene, sind durch individuelle räumliche Eigenschaften sowie die räumlichen Eigenschaften ihrer lokalen Konfiguration beschreibbar (vgl. Kap. 5.1).
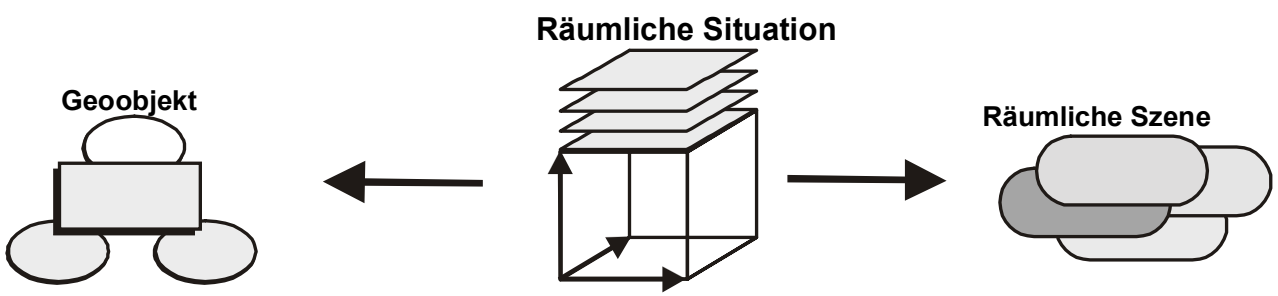

Abb. 45: Bestandteile einer räumlichen Situation.

Eine räumliche Szene beschreibt eine Konfiguration mehrerer Elemente einer räumlichen Situation. Die Ereignisse beschreiben den Inhalt einer Veränderung der Elemente oder der räumlichen Szene, während die Prozesse den Mechanismus der Veränderung beschreiben. Die Eigenschaften der Geoobjekte und räumlichen Szenen werden in den Kapiteln 5.1 und 5.2 weitgehend beschrieben. An dieser Stelle soll nur auf die Zusammenhänge mit räumlichen Entscheidungsproblemen eingegangen werden.

Ein Element wird durch seine räumlichen und thematischen Eigenschaften sowie ihre zeitliche Veränderung definiert. Die individuellen räumlichen Eigenschaften (Form, Größe, Ausrichtung) sind für die räumliche Entscheidungsfindung nur so weit interessant, inwieweit sie die relative Position der Elemente beeinflussen. Sie können zur Entscheidungsfindung beitragen, stellen aber kein Abgrenzungskriterium räumlicher Entscheidungsprobleme dar. Die individuellen räumlichen Eigenschaften können jedoch für eine höhere Dimension der Kriterienausprägungen sorgen. Statt nach der ursprünglichen (eventuell sehr komplexen) Geometrie eines räumlichen Objektes kann dieses nach seiner Approximation, Teilen seiner Zerlegung oder Abstraktion (s. Kap. 5.1.2) beschrieben werden (Abbildung 46). 


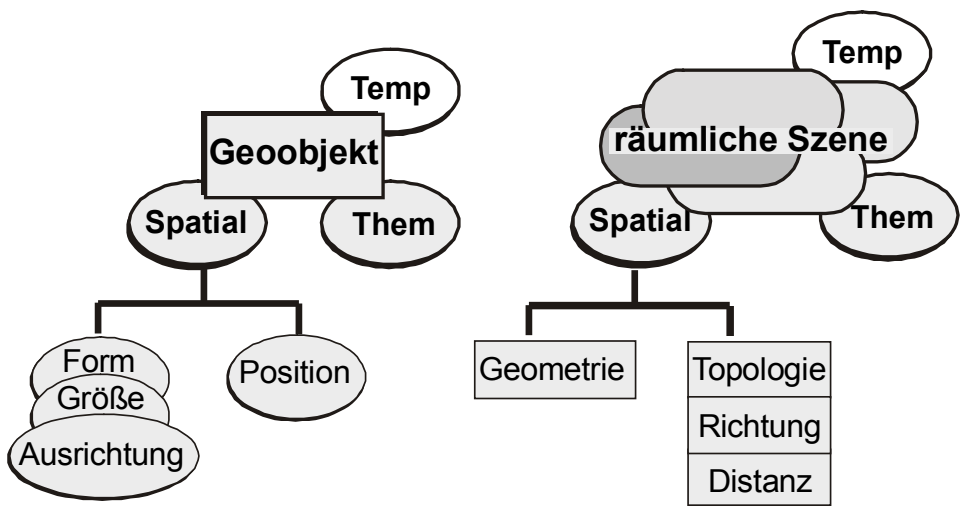

Abb. 46: Eigenschaften eines räumlichen Elementes und einer räumlichen Szene.

Die räumlichen Beziehungen entscheidungsrelevanter Objekte sind ein wesentliches Differenzierungsmerkmal der räumlichen Entscheidungssituation. Die Theorie der räumlichen Beziehungen ist daher für die räumliche Entscheidungsfindung von großer Bedeutung: Sie untersucht Beziehungsarten, welche in der räumlichen und insbesondere geographischen Domäne zur Beschreibung der relativen Lage angewendet werden können. Individuelle Beziehungen wie Topologie, Distanz und Richtung beschreiben die paarweisen Beziehungen der Elemente einer räumlichen Situation, während die räumliche Verteilung und Muster die Anordnung der Elemente in einer räumlichen Szene aggregiert beschreiben (Abbildung 47).

Integration individueller Beziehungen sowie Ableitung der unbekannten Beziehungen ist das Thema von räumlicher relation algebra. Sie ermöglicht die Verarbeitung räumlicher Szenen. Die Ähnlichkeit der Beziehungen, gemessen mit Hilfe von qualitativen oder quantitativen Konzepten, ist für den Vergleich und die Anordnung räumlicher Alternativen unerlässlich.
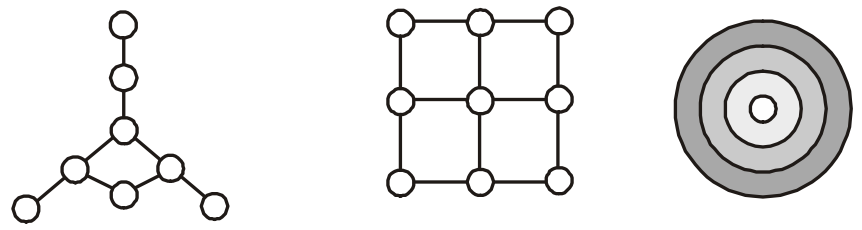

Abb. 47: Beziehungsarten einer räumlichen Situation: topologische Beziehungen, Richtungsbeziehungen und Distanzbeziehungen.

Auch zeitliche Änderung der Beziehungen mit zugrundeliegenden Ereignissen und die zeitlichen Beziehungen der Ereignisse sind für die räumliche Entscheidungsfindung wichtig. Sie beschreiben die Dynamik der räumlichen Situation aus der Sicht der Handlungsmöglichkeiten zur Beseitigung eines Entscheidungsproblems.

Verschiedene an einer räumlichen Entscheidungssituation beteiligte Akteure können unterschiedliche Konzepte zur Entscheidungsfindung anwenden. Die Modellierung einer räumlichen Situation setzt eine Integration von unterschiedlichen Konzeptualisierungen voraus. In Abbildung 48 ist der Prozess einer solchen Integration schematisch dargestellt. 


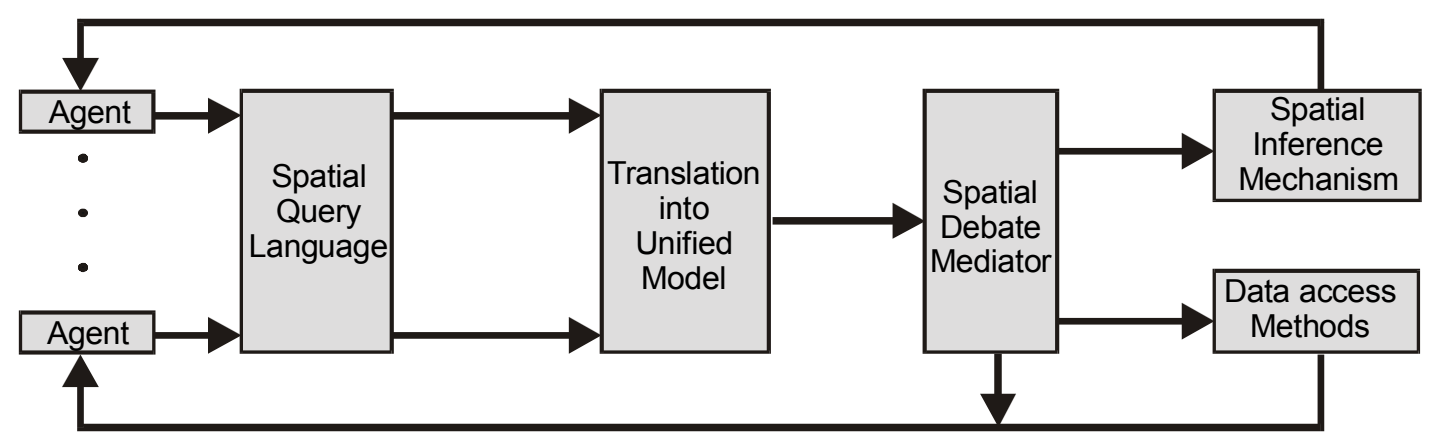

Abb. 48: Integration von unterschiedlichen Konzepten einer räumlichen Situation (nach PAPADIAS \& EGENHOFER 1996b)

Die Entscheidungsträger (Agenten) modellieren ihre eigene räumliche Situation als Grundlage eines Entscheidungsproblems. Ihre Konzepte werden verglichen und in ein einheitliches Modell überführt. Vor allem qualitative Beziehungen (nah, weit, nördlich, westlich, in, außerhalb) können unterschiedliche Bedeutung für die Agenten besitzen. Die Güte einer solchen Angleichung wird von einem Vermittler (Mediator) während einer Rückkopplung mit den Agenten überwacht. Liegt eine einheitliche und widerspruchsfreie räumliche Situation vor, so kann die Entscheidungssuche fortgesetzt werden.

\subsubsection{Räumliche Entscheidungssituation}

Eine räumliche Situation wird durch Festlegung von Alternativen und Kriterien zur räumlichen Entscheidungssituation erweitert.

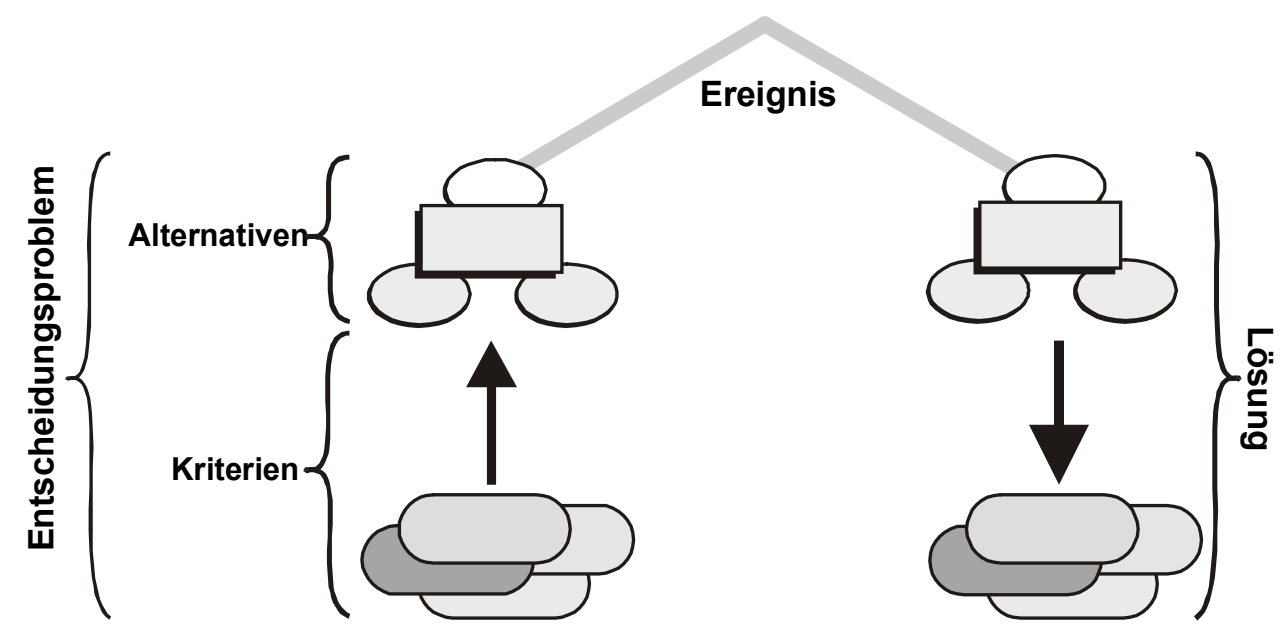

Abb. 49: Bestandteile räumlicher Entscheidungssituationen.

Zur Lösung eines räumlichen Problems wird ein räumliches Objekt und/oder ein Ereignis gesucht, durch welches die räumliche Situation der Zielvorstellungen des Entscheidungsträgers am besten angeglichen wird. Das zu ändernde räumliche Objekt zusammen mit dem Ereignis seiner zielgerichteter Änderung stellt eine Alternative dar (Abbildung 49). Zu ihrer Bewertung werden räumliche Beziehungen $\mathrm{zu}$ anderen entscheidungsrelevanten räumlichen Objekten herangezogen. 
Eine Alternative ist ein räumliches Objekt, welches eine Handlungsmöglichkeit zur Behebung des Entscheidungsproblems räumlich repräsentiert. Sie setzt sich aus den Elementen und den Ereignisse zusammen, die zur Lösung des Entscheidungsproblems führen können (Abbildung 49).
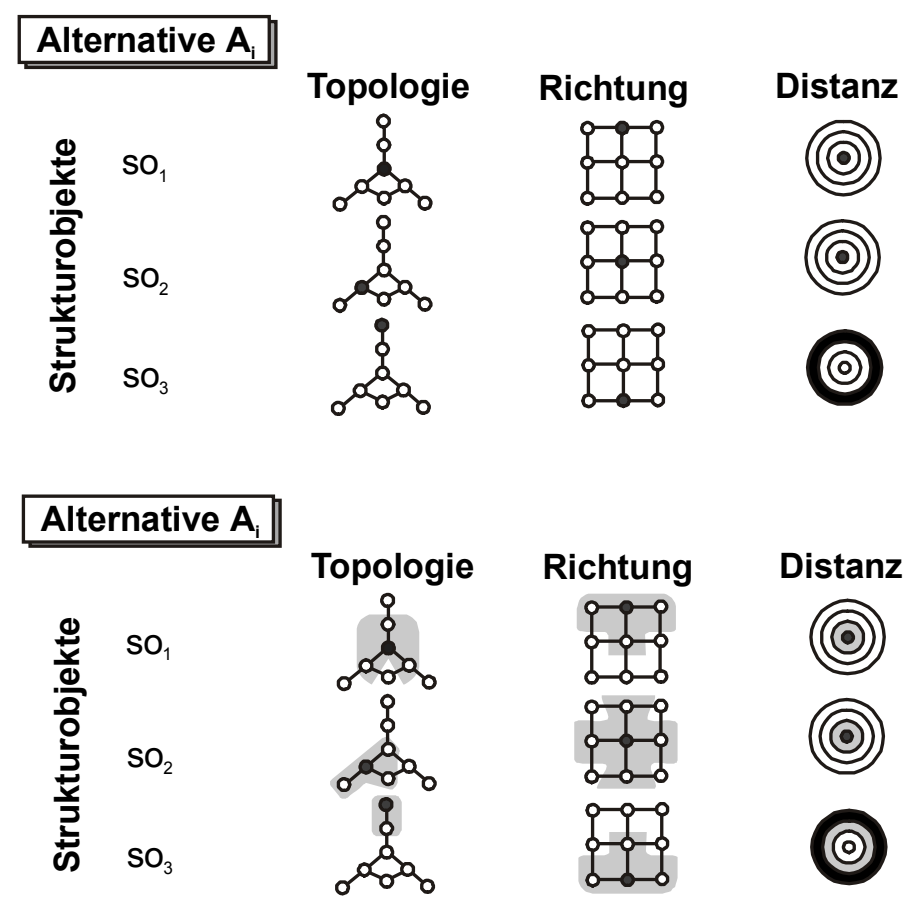

Abb. 50: Räumliche Beziehungen als Ausprägungen räumlicher Kriterien: (oben) sichere Beziehungen; (unten) unsichere Beziehungen in konzeptioneller Nachbarschaft.

Der positionale Aspekt der Alternativen wird durch eine oder mehrere Mengen der räumlichen Objekte definiert, die sog. Strukturobjekte. Die Strukturobjekte sind Mengen, die in einer bewertungs- oder präferenzabbildenden Beziehung zu der Menge der räumlichen Alternativen stehen.

Ein Entscheidungskriterium ist eine auf das verfolgte Ziel des Entscheidungsproblems bezogene Eigenschaft der Alternativen, anhand welcher die räumliche Präferenz des ETs abgebildet wird. Die Entscheidungskriterien können eine thematische oder geometrische Komponente betreffen. Sie setzten sich aus den Strukturobjekten und ihren Beziehungen zu den Alternativen zusammen (Abbildung 49).

Bei der Festlegung eines Entscheidungskriteriums sind drei Schritte zu unterscheiden. Es muss eine Eigenschaft der Alternativen gefunden werden, nach welcher der Zielerfüllungsgrad beurteilt werden kann. An dieser Eigenschaft werden Äquivalenzklassen definiert. Eine Äquivalenzklasse stellt eine Menge der Ausprägungen eines Entscheidungskriteriums dar, die aufgrund ihrer Ähnlichkeit gleich zur Erfüllung des Zieles beitragen. Im letzten Schritt müssen die Äquivalenzklassen präferenzorientiert geordnet werden.

Die Kriterien unterscheiden sich u.a. nach ihrem Kompensationscharakter. So können Kriterien für die Ausscheidung der Alternativen (Alternativenbedingungen, constraint) und Kriterien für Alternativenbewertung (Bewertungskriterien) unterschieden werden. Während die constraints die 
unzulässigen potentiellen Alternativen ausschließen, dienen die Bewertungskriterien zu einer Alternativenordnung. Die constraints (auch als harte Kriterien bezeichnet) lassen keine Kompensation von schlechten Werten durch sehr gute Ausprägung in einem anderen Attribut zu. Die Bewertungskriterien erlauben, die Menge der effizienten Lösungen zu bestimmen und sind Ausgangspunkt der Präferenzaggregation. Sie lassen eine Kompensation grundsätzlich zu, wobei die Kompensation von der Entscheidungsregel bestimmt wird. Werden in einer Entscheidungsregel Mindestgrenzen eines Bewertungskriteriums festgelegt, bezeichnet man sie als satisfaction constraint. Die Bewertungskriterien beschreiben die in früheren Schritten definierten Alternativen und ermöglichen, die effizienten von den unzulässigen oder dominierten zu trennen.

Die Beziehungen der Alternativen zu ihren Kriterien können zur Beschreibung räumlicher Entscheidungsprobleme herangezogen werden: Statische Alternativen sind Alternativen, welche der selben zeitlichen Periode angehören wie die räumlichen Kriterien (Abbildung 51a). Bei dynamischen Alternativen gehören die Kriterien $\mathrm{zu}$ mehreren unterschiedlichen zeitlichen Perioden, während die Alternativen nur einer zeitlichen Periode angehören. Gehören die Alternativen einer räumlichen Entscheidungssituation $\mathrm{zu}$ unterschiedlichen Zeitperioden, so bilden diese eine Strategie, d.h. eine zusammengesetzte Alternative, deren elementare Entscheidungen in Interdependenzen zueinander stehen (Abbildung 51c). Eine Strategie liegt dann vor, wenn die Alternativen einer Entscheidungssituation Strukturobjekte einer anderen bilden. Die Lösung einer Fragestellung in einem solchen Fall schränkt die möglichen Lösungen der anderen Fragestellung ein.

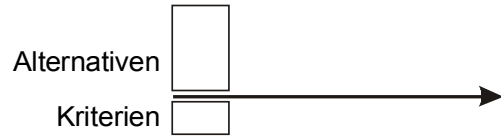

a)

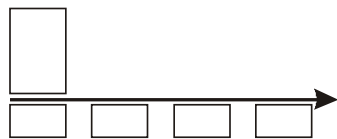

b)

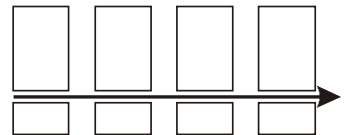

c)

Abb. 51: Zusammensetzung der Entscheidungsprobleme: a) statische Alternativen, b) dynamisches Entscheidungsproblem, c) Strategie.

In einer Entscheidungssituation wird zwischen dem Entscheidungsraum (decision space) und dem Ergebnisraum (decision outcome space) unterschieden (MALCZEWSKI et al. 1999). Der erste ist der Definitionsbereich der Alternativen, der zweite der Definitionsbereich der Kriterien. Die räumlichen Entscheidungsprobleme zeichnen sich dadurch aus, dass der geographische Raum den Entscheidungsraum darstellt (Abbildung 52).

Multikriterielle Entscheidungsfindung kombiniert bei der Präferenzabbildung tabellarische Ergebnisse mit graphischen Visualisierungstechniken. Dabei können Methoden angewendet werden, die ursprünglich für die statistische multivariate Datenexploration entwickelt wurden (Histogram, Scatterplot, Profile, Icongrafiken wie Chernoff Gesichter, Andrews Kurven, etc.). Während diese in der Statistik zum Finden von Clustern ähnlicher Daten, zur Detektion von Ausreißern und untypischer Daten oder zur Charakterisierung der Variabilität angewendet werden, ist ihr primäres Ziel in der multikriteriellen Analyse die Untersuchung der Kompensation (Trade-off) zwischen konfliktären Kriterien und die Suche nach einer ,optimalen' Lösung des Entscheidungsproblems (MALCZEWSKI et al. 1997). 


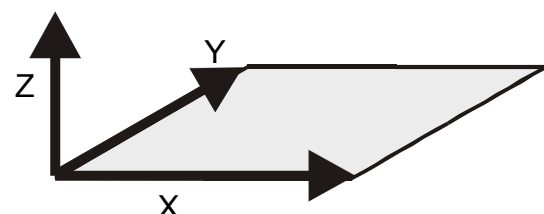

a)

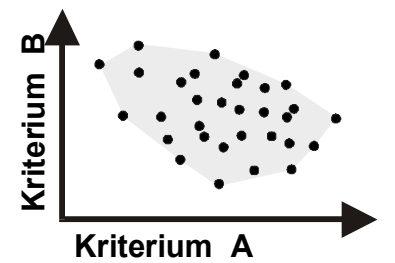

b)

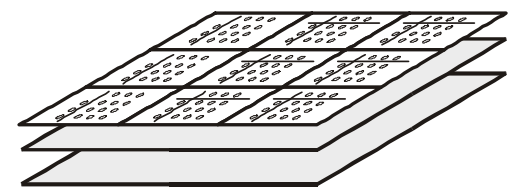

c)

Abb. 52: Entscheidungsraum (a) und Ergebnisraum (b) einer räumlichen Entscheidungssituation, schematisch dargestellt als Zusammensetzung der räumlich - referenzierten Entscheidungsprobleme (c).

Als Beispiele zur Visualisierung von Zusammenhängen in einer räumlichen Entscheidungssituation können payoff matrix und Scatterplot Kartenmatrix dienen (MALCZEWSKI et al. 1997).

Die Payoff-Matrix ist eine quadratische Matrix von individuell optimierten Kriterien (Abbildung 53). Die Matrix kann zur Aufdeckung von positiv und negativ idealen Lösungen dienen. Die Diagonale der Matrix enthält die ideale Lösung, d.h. die beste Ausprägung der separat behandelten Kriterien. Die jeweils schlechteste Ausprägung der Spalten stellt die negativ ideale Lösung dar. Die Kriterienausprägungen in der Matrix können sowohl numerisch als auch graphisch dargestellt werden.

Eine bivariate Scatterplot/Karte Matrix ist eine quadratische Matrix von paarweisen Vergleichen der Kriterien (Abbildung 53). Unter der Diagonale wird dabei der paarweise Vergleich in dem Ergebnisraum visualisiert, während oberhalb der Diagonale der Vergleich in dem geographischen Entscheidungsraum dargestellt wird.

\section{Payoff Matrix}

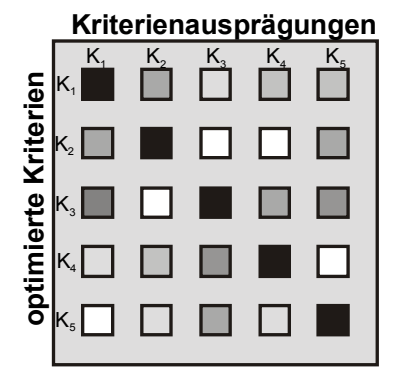

Scatterplot / Map Matrix

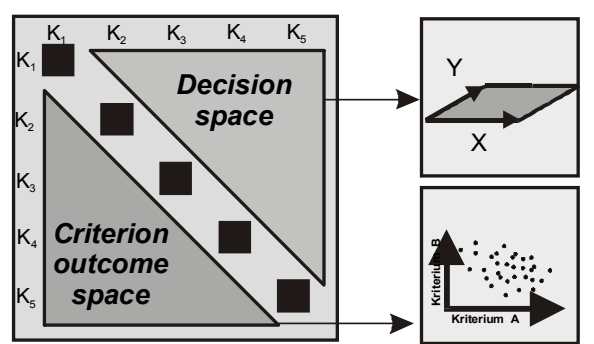

Abb. 53: Graphische Hilfsmittel zur Erforschung von Zusammenhängen in der Entscheidungssituation.

Auch weitere Methoden des visuellen Data Mining ${ }^{5}$ können zur Erforschung beider Entscheidungsräume angewendet werden.

\footnotetext{
5 Ansätze zur Entdeckung von (neuer) Struktur in sehr großen Datenbeständen: Regelmäßigkeiten, Abhängigkeiten, Trends - Informationen, die aus den ursprünglichen Gegebenheiten der Erfassung der Daten nicht direkt abzulesen sind.
} 


\subsection{Räumliche Präferenz}

Na schön soll es sein! Ein ziemlich großes Fleckchen guter Erde an einem sanften sonnigen Südhang, nicht zu nahe am Stadtzentrum, aber auch nicht sehr weit draußen. Der Straßenlärm muss weit weg und der Bach nahe, aber auch nicht zu nahe sein. Relativ billig muss das Ganze natürlich sein oder wenigstens sehr groß. Das Grundstück sollte mit dem Bus gut erreichbar sein, und mit dem Fahrrad sollte man mehr oder weniger sicher in den Park fahren können. Die Gegend im Süden gefällt mir gut aber weiter nördlich ist's auch nicht schlecht. Schön soll's einfach sein. Hast du's gefunden in deinem Computerkastl? ... Das kann doch nicht schwer sein! (REINBERG 2000)

Das tägliche Leben wird von zeitlichen und räumlichen Erwägungen stark geprägt. Fortbewegung im Raum als eine elementare räumliche Handlung geschieht als Folge vielseitiger räumlicher Überlegungen. In einfachen Situationen fällt es den Menschen leicht, zeitliche und räumliche Aufgabenstellungen zu lösen. Sie verfügen über eine Repräsentation vom räumlichen und zeitlichen Wissen, das zu schnellem und effizientem Lösen der räumlichen Alltagsprobleme eingesetzt wird. Die Menschen verwenden räumliche Metaphern dazu, auch andere Domänen zu strukturieren. Die Fähigkeit des räumlichen und zeitlichen Schließens ist eine grundlegende Fähigkeit der Menschen. Sie wird in Kombination mit räumlicher Präferenz zur Lösung räumlicher Wahl- und Entscheidungssituationen eingesetzt.

Unter einer Präferenzrelation wird ein Bewertungskriterium verstanden, das die Intensität des Strebens nach den mit der Ergebnisdefinition festgelegten Zielgrößen zum Ausdruck bringt und damit den Entscheidungsträger bei der Auswahl einer Handlungsalternative unterstützt (vgl. HÄNLE 1993). Die räumliche Präferenz ist eine Ansicht des ETs, welche eine Anordnung der Alternativen nach ihrer räumlichen Position erlaubt. Der Entscheidungsträger zieht eine Alternative nach ihrer relativen Position einer anderen vor oder bezeichnet sie als äquivalent. Räumliche Präferenz ist kein neuer Begriff: In der Mikroökonomie wird sie beispielsweise zur Definition des vollkommenen Marktes ${ }^{6}$ angewendet, in der Psychologie ist sie bei der Verhaltensuntersuchung von Bedeutung.

Das Vorhandensein einer räumlichen Präferenz im alltäglichen Leben kann mit häufigen sprachlichen Aussagen belegt werden: So drückt beispielsweise die Aussage "das ist zu weit" eine sich auf Distanzbeziehungen stützende nicht kompensierbare Präferenz aus. Die räumliche Präferenz wird in diesem Fall in Form eines Ausschlußkriteriums, einer Art Satisfaktionsbedingung geäußert. In solcher Situation wird oft die momentane Position des Entscheidungsträgers (subjektive "Hier" Raumorientierung) als räumliches Objekt angewendet, das zur Bewertung von Alternativen dient. In dem Zitat am Anfang des Kapitels wird ein räumliches Entscheidungsproblem aus der Sicht eines Wohnungssuchenden vorgestellt. Eine "optimale" Wohnung muss bezüglich ihrer relativen Position mehrere Kriterien erfüllen, welche anhand räumlicher Beziehungen beschreibbar sind. Die Kriterien enthalten darüber hinaus

${ }^{6}$ Die Definition des vollkommen Marktes geht u.a. davon aus, dass kein Nachfrager einen Anbieter aufgrund seines Standortes vorzieht, dass also keine räumliche Präferenz vorliegt (STOBBe 1991). 
unscharfe Äußerungen, welche die Präferenzabbildung zusätzlich erschweren. Die räumliche Präferenz kann mit Hilfe der positionsbezogenen Konzepte abgebildet und gemessen werden. Die Anwendung der räumlichen Konzepte bei der Modellierung der räumlichen Präferenz stellt eine Verbindung zur GIScience dar.

Die räumliche Präferenz kann zur Abgrenzung räumlicher Entscheidungsprobleme sowie zur Strukturierung räumlicher Entscheidungssituation beitragen. Sie kann ebenfalls zur Strukturierung anderer Präferenzarten (Risikopräferenz und Zeitpräferenz) beitragen: Ein Entscheidungsträger kann eine differenzierte Risikopräferenz bezüglich der räumlichen und thematischen Unsicherheit besitzen.

Die Position der räumlichen Präferenz im Präferenzsystem der Entscheidungstheorie ist bisher kaum erforscht. FRANCK (1997) geht auf die Ähnlichkeit von Zeit und Raum ein: aufgrund der subjektiven Zentrierung des Raumes und der Zeit sind die dem Zentrum (HIER und JETZT) nahe gelegenen Positionen wichtiger als die ihm fernen. Dementsprechend ist das Gegenteil von Diskontierung der 'zeitlichen Entfernung' mit Hilfe von Zinsen die 'räumliche Diskontierung' der räumlichen bzw. geographischen Entfernung. Im Unterschied zur Zeit besitzt der Raum kein objektives Zentrum und keine objektive Richtung. Es gibt also keinen einheitlichen Ausgangspunkt der Diskontierung und die Rate der Diskontierung kann je nach dem Standpunkt und von jedem Standpunkt nochmals nach jeder Richtung unterschiedlich ausfallen (FRANCK 1997). Dennoch kann eine Struktur der 'räumlichen Diskontraten' z.B. in der räumlichen Struktur von Grundstückpreisen beobachtet werden: "Ein Grundstück mit Seeblick ist teuerer als ein sonst gleiches, an dem man von der Nähe der Attraktion nichts spürt. ...Umgekehrt sinkt der Wert eines Grundstückes, wenn sich schmutzige, hässliche oder sonst störende Nutzung in der Nachbarschaft breit macht" (FRANCK 1997).

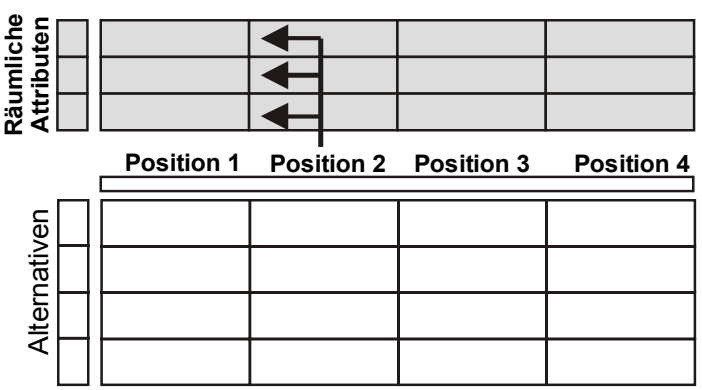

a)

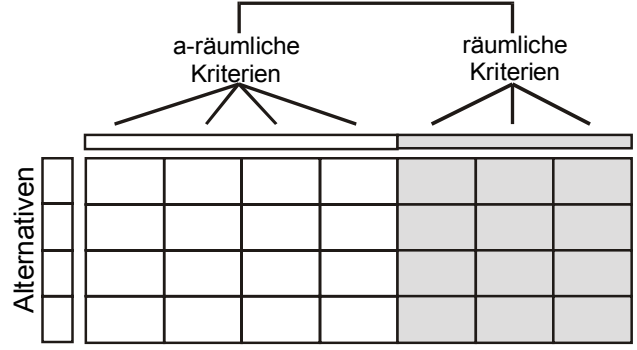

b)

Abb. 54: Modellierung räumlicher Präferenz: a) als Artenpräferenz ähnlich wie bei der zeitlichen Präferenz; b) als kombinierte Höhen- und Artenpräferenz der nicht-räumlichen räumlichen Kriterien.

Wegen der Ähnlichkeit der zeitlichen und räumlichen Präferenz liegt nahe, die räumliche Präferenz, ähnlich wie im Fall der zeitlichen Präferenz, als Artenpräferenz zu modellieren, indem man jede räumliche Position als ein Entscheidungskriterium betrachtet (Abbildung 54a). Wegen der objektiven Zentrums- und Ausrichtungslosigkeit des Raumes könnte man diese, wie es in Abbildung 54a dargestellt wird, nur anhand mehrerer Kriterien gewinnen. Da der Mensch nicht gegenüber absoluten (xyz Koordinaten) sondern gegenüber relativen Positionen (in Beziehung zu räumlichen Strukturobjekten) des Raumes eine Präferenz besitzt, würde die Anwendung der 
räumlichen Präferenz als Artenpräferenz ein assoziiertes Entscheidungsproblem enthalten (Abbildung 54b).

Zur Lösung räumlicher Entscheidungsprobleme ist die räumliche Präferenz nicht in jedem Fall unerlässlich. Die Lösung von räumlichen Problemen kann dadurch gefunden werden, dass die Räumlichkeit in eine andere Größe (beispielsweise Kosten oder Dauer des Transportes) überführt wird. Ist das in einer räumlichen Entscheidungssituation bei allen Kriterien möglich, so führt diese Transformation zur Lösungsfindung. Eine solche Transformation kann aber gleichzeitig ein Risiko verbergen, die räumliche Präferenz nur teilweise oder gar ungeeignet auszudrücken. Dies kann dazu führen, dass die Relationen über Alternativen anhand der transformierten Größe zu Intransitivitäten neigen. Ebenfalls kann die räumliche Präferenz versteckte Präferenzen anderer Arten enthalten.

Räumliche Entscheidungssituationen mit ausschließlich räumlichen Zielen, sicheren Informationen und statischem Zeitbezug zeichnen sich durch das Vorhandensein nur räumlicher Präferenzen des ETs aus. Die Alternativen werden nur nach ihrem Raumbezug (Position) bewertet und angeordnet. An die räumliche Präferenz werden ähnliche Anforderungen gestellt, wie an die übrigen Präferenzarten. Die rationalen Entscheidungen setzen Vollständigkeit und Transitivität der räumlichen Präferenzrelation voraus. Die Vollständigkeit verlangt, dass jedes Alternativenpaar miteinander vergleichbar ist. Die Transitivität verlangt elementare Komposition der Präferenzbeziehungen. Sind die räumlichen Präferenzrelationen vollständig und transitiv, so können sie durch eine Funktion ersetzt werden. Eine Präferenzfunktion $\Phi_{\mathrm{s}}$ ist eine Funktion, die jeder Alternative einen Präferenzwert zuordnet, deren numerische Beziehungen zu den Präferenzrelationen über die Alternativen äquivalent sind. Um kardinale Aussagen über die Differenzen der Präferenzwerte der Alternativen $\mathrm{zu}$ erlauben, muss die Bedingung der vollständigen und transitiven Ordnung auch für sie gelten.

Die räumliche Präferenz kann selten nur durch ein Kriterium bzw. Ziel ausgedrückt werden. Diese Eigenschaft ist den inhärenten Eigenschaften des Raumes zuzuschreiben. Bei mehreren räumlichen Kriterien kann es zu einer Zielkonkurrenz kommen, wenn die bessere Erfüllung eines Zieles die Erfüllung eines zweiten vermindert. Eine Aggregation der räumlichen Präferenz, die mit Hilfe mehrerer Kriterien angegeben wird, stellt neue Anforderungen an die Abbildung der Präferenz. Eine einfache additive Form der Aggregation setzt die Präferenzunabhängigkeit der Kriterien voraus. Dies meint, dass die Präferenz anhand zweier Kriterien nicht von der Ausprägung eines dritten Kriteriums abhängen darf, sofern diese bei den Alternativen gleich ist (s. Kapitel 6.6). Die messbare additive Form der Präferenzaggregation verlangt noch die Präferenzunabhängigkeit bezüglich der Differenzen der Präferenzwerte. Daraus folgt, dass die räumliche Präferenz die Axiome der Höhen- und Artenpräferenz erfüllen muss.

Die Präferenzabhängigkeit ist wegen der Vielfältigkeit möglicher Interdependenzen eher die Regel. Die Entdeckung versteckter Präferenzen kann mit Hilfe von Verzerrung erreicht werden. Die räumliche Szene wird auf präferenzunabhängige Weise verzerrt und der ET wird nach der Änderung seiner Präferenz gefragt. Auch die Überprüfung von schon getroffenen Lösungen kann auf diese Weise vorgenommen werden.

Die räumliche Präferenz kann allein oder in Kombination mit anderen Präferenzarten (Zeitpräferenz, Risikopräferenz) zur Lösung eines Entscheidungsproblems herangezogen werden. Die Kombination der Präferenzen erfordert immer Vergleich und Abwägung der 
zugrundeliegenden Ziele. Besteht zwischen den Zielen eine Konkurrenz, dann hat eine bessere Erfüllung eines Zieles die Verschlechterung der anderen zur Folge. In einer Situation, in welcher der Entscheidungsträger sowohl eine räumliche als auch eine a-räumliche Präferenz besitzt, ist eine Lösungsfindung nur durch eine geeignete Kombination der beiden möglich. Sind die zugrundeliegenden Ziele kompensierbar, so ist der ET bereit, auf die besseren Konsequenzen einer Alternative bezüglich der a-räumlichen Ziele zu Gunsten der besseren Konsequenzen der räumlichen Ziele zu verzichten und umgekehrt.

\subsection{Räumliche Entscheidungsmodelle}

Zur Lösung räumlicher Entscheidungsprobleme haben sich vor allem multikriterielle Methoden der Entscheidungstheorie als geeignet erwiesen (s. Kap. 6.7).

Zur Formalisierung der Zusammenhänge innerhalb der Entscheidungsmodelle wurden mehrere Ansätze entwickelt (MALCZEWSKI 1996, JANKOWSKI 1995, KARACAPILIDIS et al. 1999, EASTMAN 1999, JANG \& EASTMANN 2000). Diese gehen davon aus, dass die Darstellungsweise des Grundmodells der Entscheidungstheorie an die räumlichen Fragestellungen angepasst werden muss. Das Modell der räumlichen Entscheidungsfindung umfasst demnach die Strukturierung und Repräsentation einer Entscheidungssituation bezogen auf eine räumliche Fragestellung während des Entscheidungsprozesses.

Die räumlichen Entscheidungsprobleme können nach der von der Entscheidung betroffenen Komponente in positionale und thematikbezogene Entscheidungsprobleme gegliedert werden. Während bei den ersten die Position der Gegenstand der Entscheidung ist, ändert sich die Position bei der zweiten Klasse nicht, lediglich die thematischen Eigenschaften werden von der Entscheidung betroffen. Die Änderung einer thematischen Eigenschaft der Alternativen als Ziel eines räumlichen Entscheidungsproblems setzt voraus, dass die (betroffenen) thematischen Attribute eine Auswirkung auf die geometrisch-thematischen Beziehungen haben. Die räumlichen Fragestellungen können demnach in zwei Klassen gegliedert werden: Position / Assignment.

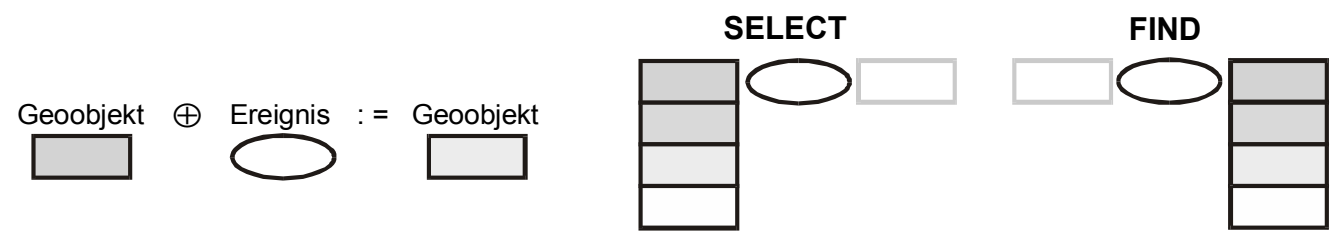

Abb. 55: Schematische Darstellung der positionalen Entscheidungsprobleme.

Allen positionalen räumlichen Entscheidungen liegt eine elementare Fragestellung SELECT oder FIND zugrunde (Abbildung 55). Die objektbezogenen Entscheidungssituationen (SELECT) bezeichnen die Suche nach einem Geoobjekt. Die Alternativenmenge ist explizit bekannt und wird aus vorhandenen Objekten konstruiert. Die feldbezogenen Fragestellungen (FIND) beschreiben eine Suche nach der Position. Die Alternativen sind nicht explizit bekannt, sie müssen zuerst generiert werden. Diese Gliederung folgt somit der Identität von den der Alternativen zugrundeliegenden Geoobjekten: Die Fragestellung SELECT betrifft existierende 
Elemente mit eigener Identität, während FIND die Bildung von Elementen mit einer neuen Identität beabsichtigt.

Die thematikbezogene Fragestellung (ASSIGNMENT) bezeichnet eine Situation, in welcher die Position der Alternativen nicht eindeutig ist, sie kann von mehreren (oder allen) Alternativen geteilt werden. Der Gegenstand der Entscheidung ist eine eindeutige Zuordnung der thematischen Eigenschaften zu den räumlichen Komponenten der Alternativen.

Die elementaren räumlichen Fragestellungen können, der Zusammensetzung räumlicher Alternativen zufolge, nach den zugrundeliegenden Prozessen oder Ereignissen weiter spezialisiert werden. In Tabelle 16 werden einige Ereignisse aufgelistet, welche als Bestandteil der Alternativen angewendet werden können. Diese sind nach der Komponente eines Geoobjektes gegliedert, welche sie betreffen können.

Tab. 16: Individuelle Ereignisse der Entscheidungsprobleme.

\section{räumliche Komponente}

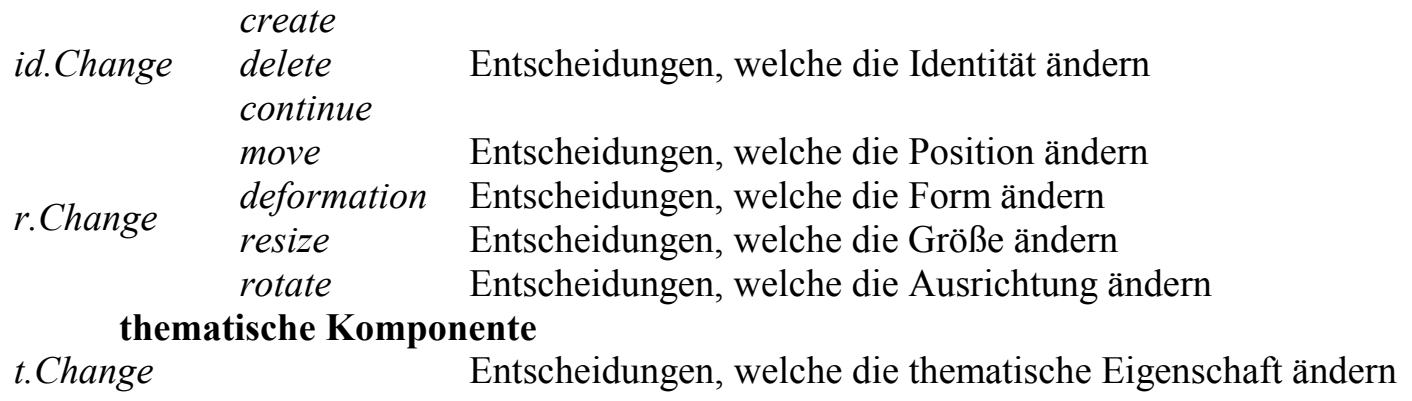

Durch die Ereignisse der Klasse id.Change wird ein Objekt mit einer neuen Identität gebildet (create), oder eines mit vorhandener Identität gelöscht (delete). Das Ereignis continue bezeichnet die weitere Existenz eines Geoobjektes mit unveränderter Identität. Bei den Ereignissen create und delete können aufgrund der geänderten Identität Beziehungen jeder Art geändert werden. Die geometrische Komponente der Strukturobjekte bleibt unverändert.

Die Klasse $r$.Change fasst die Ereignisse zusammen, durch die Änderungen der geometrischen Komponente (Position, Form, Größe, Ausrichtung) der ausgewählten Alternative erfolgen. Neben den individuellen Änderungen können auch Änderungen in der lokalen Konfiguration der Alternativen vorkommen (s. Tabelle 17).

Tab. 17: Ereignisse, welche die lokale Konfiguration der Alternativen ändern (in Anlehnung an ClarAmOUNT \& THÉRIAUlT 1995).

re-allocation $\quad$ Eine Änderung der Alternative ändert auch die geometrische Komponente eines in der lokalen Konfiguration befindlichen SO

split Durch die Änderung der Alternative entsteht in ihrer lokalen Konfiguration mindestens ein neues Element

union Durch die Änderung der Alternative verliert in ihrer lokalen Konfiguration mindestens ein vorhandenes Element seine Identität

Unter t.CHANGE sind Ereignisse zusammengefasst, bei welchen ein thematisches Attribut der ausgewählten Alternative geändert wird. 
Eine SELECT Entscheidung kann mit Ereignissen verbunden werden, die jede ihrer Komponenten betreffen. Ist der Output eines Ereignisses nicht vorgegeben, kann an seine Stelle die Entscheidung eines anderen Submodells gesetzt werden.

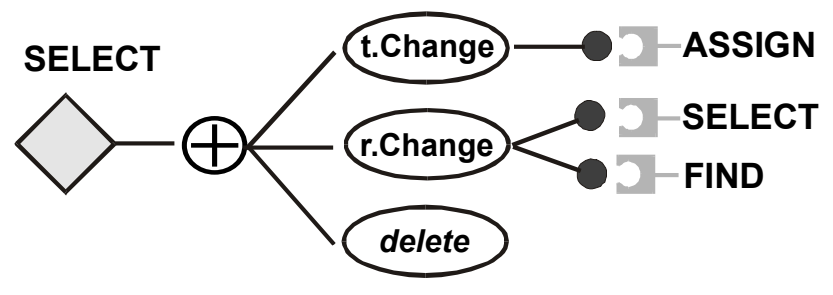

Die Entscheidung (SELECT ; t.Change) richtet sich nach den thematischen Eigenschaften der Alternativen. Die Auswahl der Alternative verfolgt das Ziel, eine ihrer Attributsausprägungen zu ändern oder neu zuzuordnen. Eine Strategie dieser Entscheidungsart bezeichnet eine Menge der aufeinander folgenden elementaren Entscheidungen in der gleichen oder in unterschiedlichen Perioden. Die zuzuordnende thematische Eigenschaft kann von dem Entscheidungsträger vorgegeben oder mit Hilfe einer ASSIGN Entscheidung bestimmt werden.

Die Entscheidung (SELECT ; r.Change) beabsichtigt die Änderung der geometrischen Komponente von der Alternative zugrundeliegenden Zielobjekten. Die neue Ausprägung eines geometrischen Attributes kann vom Entscheidungsträger vorgegeben werden oder mit Hilfe der Entscheidung FIND gefunden werden.

Die Entscheidung (SELECT ; delete) hat den Verlust der Identität von Geoobjekten, die eine Alternative bilden, zum Ziel. Die räumliche Komponente der Zielobjekte wird dem Zwischenraum der räumlichen Situation zugeordnet. Das Ereignis delete benötigt keinen entscheidungsrelevanten Output.

Die Entscheidung (FIND ; create) bezeichnet eine Entscheidung, deren Ziel die Bildung eines Geoobjekts mit neuer Identität ist. Von dieser Entscheidung können alle räumlichen Attribute und ihre Kombinationen betroffen werden (Form, Größe, Ausrichtung und Position).

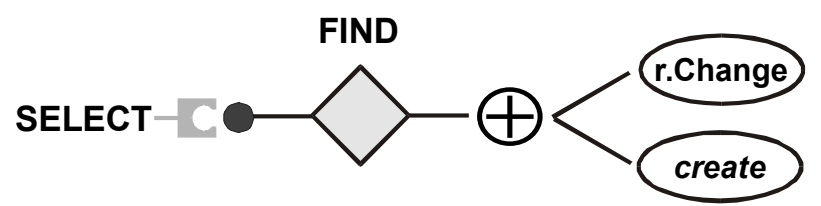

Die Entscheidung (FIND ; r.Change) hat zum Ziel, die geometrische Komponente eines vorgegebenen oder mit Hilfe von SELECT gefundenen Zielobjekts zu ändern. Neben den individuellen Änderungen eines geometrischen Attributs (move, deformation, resize, rotation) kann auch ihre Kombination das Ziel der Entscheidung sein. Die Änderung der morphologischen Eigenschaften der aneinander grenzenden Objekte einer lokalen Konfiguration ist komplizierter als in dem Fall allein stehender Objekte. Jede Veränderung einer morphologischen Eigenschaft eines Objektes verursacht eine Veränderung von mindestens einem Objekt in der lokalen Anordnung des geänderten Objektes. Das Ergebnis einer solchen Änderung kann mit drei Veränderungen der lokalen Konfiguration beschrieben werden (split, merge, re-allocation). 


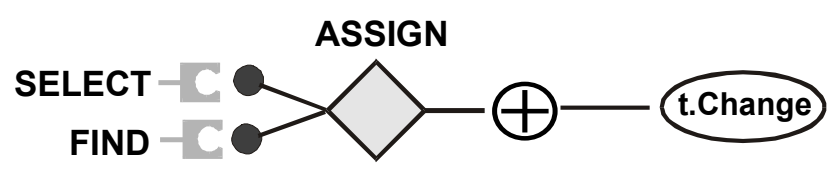

Die Entscheidung (ASSIGN ; t.Change) besteht aus der Bestimmung der Zuordnungen von thematischen und geometrischen Eigenschaften zu den ZO Objekten oder Punkten des Raumes, so dass die constraints (C) und satisfaction constraints (SC) erfüllt werden. Ist eine solche Zuordnung in einer Periode nicht möglich, besteht das Problem aus der Bestimmung einer Reihenfolge der zu erfüllenden constraints in verschiedenen zeitlichen Perioden.

\subsection{Spatial Decision Support System}

Ein räumliches Entscheidungsunterstützungssystem (spatial decision support system SDSS) beinhaltet alle Komponenten eines DSS, erweitert um die Verwaltung und Manipulation von geographischen Daten, normalerweise im Kontext eines GIS. Wird ein DSS für einen Themenbereich mit räumlicher Dimension entwickelt (d.h. eins oder mehrere Merkmale mit einer räumlichen Verteilung sind bei der Problemlösung zu beachten) oder hat die Lösung, die mit dem DSS erarbeitet wird, einen räumlichen Bezug, so kann das analytische System als Spatial Decision Support System bezeichnet werden.

"SDSS is an interactive, computer-based system designed to support a user or group of users in achieving a higher effectiveness of decision making while solving a semi-structured spatial decision problem" (MALCZEWSKI 1998).

Die Aufgabe der Entscheidungsunterstützung wurde schon in früheren Zeiten dem GIS zugeordnet. GRIMSHAW (1994) definiert beispielsweise GIS als "a group of procedures that provide data input, storage and retrieval, mapping and spatial analysis for both spatial and attribute data to support the decision making activities of the organisation". Die von GIS momentan geleistete Entscheidungsunterstützung kann jedoch nur für Entscheidungsunterstützung im weiteren Sinne zutreffen (JANKOWSKI 1995, ENACHE 1994).

Das data-information-knowledge-intelligence Paradigma (HARRIS et al. 1992) zeigt eine Abgrenzungsmöglichkeit eines (S)DSS von verwandten Technologien. Die Daten mit zweckbezogener Semantik werden in Informationen konvertiert, die wiederum mit Hilfe der Ursache-Wirkung-Analyse in Wissen überführt werden können. Wissen wird zur Intelligenz, wenn es in neue Ideen integriert wird, die eine zielbewusste Zukunftsvorstellung erlauben. "In this perspective, integrating GIS, DSS and spatial analysis into SDSS constitutes a step towards converting knowledge into intelligence" (ENACHE 1994).

In einem SDSS muss jedoch die Funktionalität eines DSS um bestimmte Fähigkeiten erweitert werden. So sind ein Mechanismus für den Input von räumlichen Daten, eine Repräsentation räumlicher Beziehungen und Strukturen, analytische Techniken räumlicher und geographischer Analyse sowie kartographische Darstellung der Ergebnisse in einem solchen System unerlässlich. Ein SDSS wird daher als ein System verstanden, das aus vier Komponenten konstruiert wird:

- Datenmanagement-System (spatial Data Base Management System), 
- analytische Modellierungsfähigkeiten und analytische Prozeduren (Model Base Management System MBMS),

- Display und Berichtgenerator,

- Benutzeroberfläche

Bezüglich der Abgrenzung der Komponenten gibt es jedoch auch alternative Vorschläge (vgl. DENSHAM 1991, CZERANKA 1997).

Ein SDSS umfasst ähnlich wie ein DSS drei technologische Ebenen (ENACHE 1994). Eine SDSS toolbox ist eine Komponentensammlung von Hardware und Software, die in einer Komposition zur Bildung mehrerer Systemmodule führen kann. Ein SDSS Generator stellt eine Menge der Module dar, mit deren Hilfe man ein spezifisches SDSS generieren kann. Ein spezifisches SDSS ist eine Anwendung der Technologie (vgl. SpRAgUE, zitiert nach DenSHAM 1991).

DenSHAM (1991) unterscheidet 5 funktionale Aufgaben in der Technologie. Der SDSS toolsmith entwickelt neue Werkzeuge für die SDSS toolbox. Der technical supporter fügt neue Komponenten zum SDSS Generator hinzu. Der SDSS builder versammelt Module in einen spezifischen SDSS. Der Vermittler (intermediary) sorgt für die physische Interaktion mit dem System. Der Entscheidungsträger (decision maker) ist für die Entwicklung, Implementierung und Steuerung der ausgewählten Lösung verantwortlich.

Die Architektur eines räumlichen Gruppenentscheidungsunterstützungssystems (collaborative spatial decision support systems) ist grundsätzlich die gleiche wie die von SDSS mit zusätzlichen Komponenten für Kollaboration. Diese muss Zusammenarbeit mit verteilten Daten erlauben, interaktive Entwicklung von Szenarien und Kriterien unterstützen, sowie Bewertung der Szenarien und Anpassung ihrer Komponenten gewährleisten, bis schließlich alle Kriterien zutreffen. Besonders wegen fortschreitender Durchsetzung partizipativer Planungs- und Entscheidungsmethoden erlebt die Gruppenzusammenarbeit in GIS-Umgebungen eine rasche Entwicklung: "Some of the more interesting and exciting developments in the field of collaborative spatial decision making include the use of GIS on the Internet as a tool for public consultation and participation in the decision making processes regarding decisions of national importance" (CARVER 1996). 


\section{Anwendung der Geoinformationen zur Entscheidungsfindung in der Forstwirtschaft}

\subsection{Beitrag der GIScience zur Forstwirtschaft}

Die Methoden geographischer Informationsverarbeitung und -übermittlung helfen dabei, komplexe Zusammenhänge im Raum-Zeit-Gefüge zu überschauen, um effektiv und effizient zu handeln. Die GIScience als Wissenschaft über die Geoinformation verfügt über das Potential, die raumbezogenen Wissenschaften durch die Grundlagenforschung zu räumlichen Konzepten zu unterstützen. Mit GISystem stellt sie auch ein Werkzeug zur Anwendung und zur Forschung durch Anwendung zur Verfügung.

Die Tabelle 18 stellt die Forstwissenschaften und GIScience bezüglich des herrschenden Paradigmas gegenüber. Die GIScience baut auf dem (geographischen) Raum und räumlichen Konzepten auf. Die Position räumlicher Objekte und sich daraus ergebende vielfältige Beziehungen, räumliche Verteilungen und Muster sind dabei von Bedeutung. Wie diese von Nutzer oder Informationsgesellschaft ${ }^{7}$ wahrgenommen, abgebildet und verwendet werden, ist Gegenstand der Forschung der GIScience.

Die Forstwissenschaft stellt dem räumlichen Konzept den Nutzen als zentralen Begriff gegenüber. Nutzen in diesem Zusammenhang entsteht durch Verfügung oder Verzehr von materiellen oder immateriellen Objekten, bei welchen Bedürfnisse von Menschen entsprechend deren Zielsetzung erfüllt werden. Der einer spezifischen Zielsetzung entsprechende Nutzen in der Forstwirtschaft kann als Reaktion auf die Eingriffe in natürliche Prozesse betrachtet werden. Diese verfolgen das Ziel, die zur Verfügung stehenden Ressourcen räumlich und zeitlich umzuverteilen.

Tab. 18: Gegenüberstellung der GIScience und der Forstwissenschaft.

\begin{tabular}{c|c|c} 
Paradigma & Forstliches Paradigma & GIScience Paradigma \\
\hline zentraler Begriff & Nutzen & räumliches Konzept \\
Bestandteile & Waldökosystem & Räume \\
& Zustände / Prozesse / Interaktion & Objekte / Beziehungen \\
& Funktion & Ereignisse \\
Beschreibung & zeitliche und räumliche & Position \\
& Struktur, \\
Mittel & (Zeit-Räumliche Ordnung) & Verteilung / Muster \\
leitendes Prinzip & (Umverteilung von Ressourcen & Formulieren / Formalisieren \\
\hline
\end{tabular}

7 Geospatial Information Community (GIC) ist nach ANONYMUS (1999a1) "a collection of systems or individuals who can successfully share digital geospatial information, that is, features. This implies that the members of the community share common "chunks" of the world, definitions, interests, mutual awareness, and common technology sufficiently that they have the capability to share the information". 
Der Nutzen weist eine räumliche Verteilung auf, welche das Ergebnis von Prozessbeeinflussung und Dynamik ihrer Interaktion darstellt.

Die Zielerfüllung wird in der Forstwirtschaft in dem Gefüge von Zeit und Raum des Waldes angestrebt. Der Nutzen des Waldes ist sowohl auf die Bäume selbst, als auch auf ihre interaktive Wirkung bezogen: der Wald bildet einen Raum, dessen Nutzbarkeit durch die Anzahl der Aktivitäten bestimmt wird, die innerhalb eines zeitlichen und räumlichen Rahmens (Handlungsspielraumes) durchgeführt werden können (vgl. VoIGT 1999). Auch Konzepte von Zustand, Interaktion und Dynamik sind hierbei von Bedeutung.

Die von UCGIS formulierten Forschungsthemen der GIScience (ANONYMUS 1996) sind eine Momentaufnahme aktueller Themen dieser Wissenschaft (s. Kapitel 3.7). Zumindest indirekt können sie alle für die Forstwissenschaft von Bedeutung sein oder diese unterstützen. Im folgenden wird auf die Bedeutung einiger Themen in der Forstwissenschaft eingegangen (s. Abbildung 56).

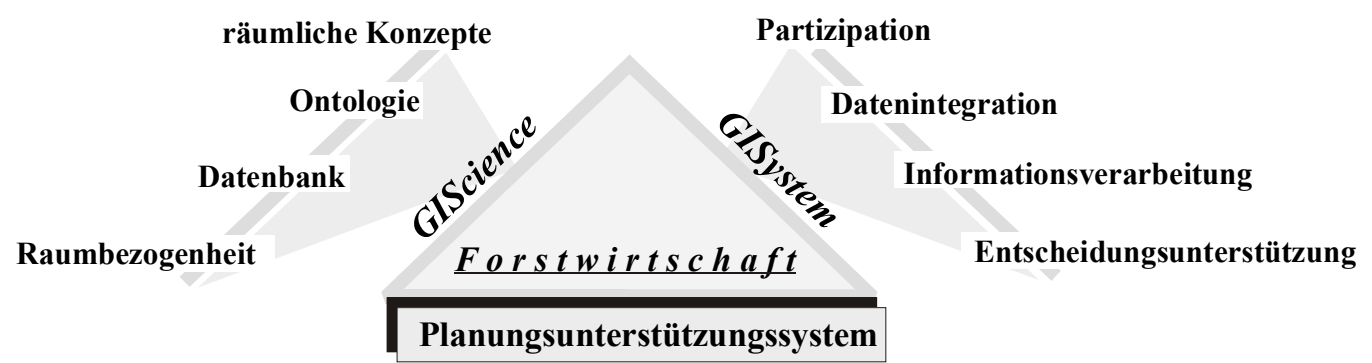

Abb. 56: Unterstützung Forstlicher Planung durch GIScience und GISystem.

Der Hauptgegenstand der GIScience ist das räumliche Konzept. Sie versucht, die räumlichen Konzepte, die in anderen Wissenschaften formuliert wurden, zu untersuchen, zu integrieren und zu ergänzen. Räumlichkeit und räumliche Konzepte sind Werkzeuge der forstlichen Planung. Mit ihrer Hilfe können Ziele verfeinert und zielgerichtete Handlungen beurteilt werden. Waren die früheren räumlichen Konzepte einfach und der mentalen Fähigkeit der Förster einfach zugänglich, so muss heute der Umgang mit ihnen durch ihre zunehmende Komplexität zumindest erst geübt werden.

Forstwirtschaft operiert mit einem komplexen räumlichen Gebilde, dem Wald. Mit seiner Ausdehnung nimmt er den geographischen Raum in Anspruch, er füllt ihn aus: „Wälder sind Räume - Räume mit besonderen biologischen Bedingungen und spezifischen, komplexen, systemaren Gesetzmäßigkeiten" (KURTH et al. 1994, S.107).

Der forstlich relevante Raum ist vielfältig: $\mathrm{Er}$ ist auf unterschiedlichen Skalen mit unterschiedlichen Objekten und Beziehungen aufgebaut. „Waldökosysteme sind hierarchische Systeme. Die sie treibenden Prozesse (Evolution, Sukzession, Systemerneuerung, Bestandesentwicklung, Bildung von Organen, Assimilation und Stoffaufnahme) laufen aufäußerst unterschiedlichen Zeit- und Raumskalen" (PRETZSCH 1997). Forst-relevante Räume sind beispielsweise:

- Der Raum eines Baumes und seiner nahen Umgebung aus der Sicht von Wachstumsbedingungen, Stabilität und Nutzung 
- Der Raum eines Bestandes und seiner nahen Umgebung für die Umsetzung individueller Ziele und Aufgaben (Detailplanung)

- Der Raum eines Bestandeskomplexes mit seinen Nachbarschaftsbeziehungen (Bereichsplanung)

- Der Raum einer Betriebsklasse als Referenzeinheit der Nachhaltigkeit (Gesamtplanung)

Die forstliche Handlung betrifft diese Räume unter vielfältiger Zielsetzung und mit unterschiedlicher Auswirkung.

In unterschiedlichen Räume können unterschiedliche Konzepte (Elemente und Beziehungen) von Bedeutung sein. Verschiedene forstlich relevante räumliche Konzepte können in der Walderneuerung, der Walderschließung, in der räumlichen Ordnung und der Waldeinteilung gefunden werden: Das Neben-, Um- und Übereinander. Räumliche Struktur in der Forstwirtschaft gewinnt an Komplexität. In den Reinbeständen konnte sie vernachlässigt werden, in den Mischwäldern ist sie eine zentrale Eigenschaft geworden: „Eine Eigenschaft von Wäldern, die nach der Umwandlung von strukturarmen Beständen verstärkt in den Vordergrund tritt, ist ihre Struktur-Determinierung. Die Bestandesstruktur stellt die Wuchsbedingungen von Einzelbaum und Bestand ein, die Wuchsbedingungen determinieren den Zuwachs, und der Zuwachs äußert sich wiederum in Strukturveränderungen von Baum und Bestand" (PRETZSCH 1997). GIScience unterstützt die Forstwirtschaft in der Aufgabe, die zielgerichteten Konzepte zu formulieren und diese zur Entscheidungsfindung einzusetzen.

Der forstliche Raum entspricht einem large-scala space (s. Kap. 3.4), er ist größer als der Mensch und seine Erforschung und Strukturierung verlangt Bewegung durch ihn. Die Regelung dieses Raumes setzt jedoch einen small-scala space voraus, in welchem die Objekte bewegt und manipuliert werden können. Die Interaktion beider Raumarten, die bei der Bildung der räumlichen Konzepte von Bedeutung ist, wird von der GIScience untersucht.

Der Räumlichkeit in der Forstwirtschaft, so wie sie die Zielsetzung und Zielerfüllung beeinflusst, wird immer mehr Aufmerksamkeit geschenkt: Welche Räume sind für die Forstwirtschaft und ihre einzelne Disziplinen von Bedeutung? Wie ist ihre Interaktion im Planungsprozess? Welche Konzepte werden in welchen Räumen und auf welchen Skalen für die Gewährleistung des Nutzens wichtig? Ist die Präferenz bezüglich dieser Konzepte räumlich ausgeprägt?

Die forst-eigenen räumlichen Konzepte prägen die Gestaltung des forstlichen Planungsmodells nicht allein. Die Komplexität der neuartigen Anforderungen an den Wald verlangt eine Integration forst-fremder Konzepte mit den forstlichen in eine nahtlose Einheit. Der Raum dient dabei als gemeinsame Referenz der vielfältigen Ansprüche an das gemeinsame Objekt des Interesses, den Wald. Die räumlichen Konzepte von Raumplanung, Landschaftsplanung und Naturschutz können dabei als Beispiele betrachtet werden. Jede dieser Disziplinen geht von eigenen räumlichen Objekten und Beziehungen aus, die durch eigene Struktur gekennzeichnet sind. Seien das Standorte, Biotope, ,patches“ oder „,corridors ${ }^{\text {“8 }}$, forstliche Planung ist mit neuartigen und fachfremden räumlichen Konzepten konfrontiert, die sie nicht entwickelt hat und für welche sie keine traditionelle Präferenz besitzt. In der Tat kommt es heute in der Forstplanung zu einer Verflechtung verschiedener Ontologien.

\footnotetext{
${ }^{8}$ Konzepte der Landschaftsplanung
} 


\section{Datenbankentwicklung}

Die mentale Verarbeitung wachsender Mengen von planungsrelevanten räumlichen Konzepten übersteigt die kognitiven Fähigkeiten des Menschen. Ihre computergestützte Verarbeitung setzt eine Transformation räumlicher Konzepte in Datenmodelle voraus. Mit wachsender Komplexität des forstlichen Planungsmodells steigen die Ansprüche an die Verarbeitung der planungsrelevanten Informationen. Die Informationen müssen in geeigneter Form gespeichert und verwaltet werden, sie müssen für die Beteiligten einfach zugänglich und beliebig strukturierbar sein. Der Aufwand zur Bereitstellung und Anwendung der Informationen im Entscheidungs- und Planungsprozess kann als Maß der Effektivität der Informationsverwaltung betrachtet werden.

Die Entwicklung einer Datenbank besteht aus der Konkretisierung der Beziehung zwischen den relevanten Aspekten der Realität und dem internen Formalismus eines Datenbanksystems. Die Phänomene und Prozesse der realen Welt werden dabei durch digitale Objekte und rechnerische Operationen repräsentiert. Die Überführung eines mentalen, räumlichen Modells der Realität in ein formales computergestütztes muss den kognitiven Fähigkeiten des Menschen entgegenkommen, sie unterstützen. GIScience begleitet also durch die Suche nach geeigneter Repräsentation und ihrer Implementierung die Bildung einer forstlichen Datenbank.

Bei der Datenbankentwicklung stellen sich vor allem zwei Problembereiche, welche die Pflege und den Austausch der Informationen betreffen:

- Nachhaltiges Datenmanagement

- Interoperabilität der Geoinformation

Unter nachhaltigem Datenmanagement wird die „Aufbereitung, Dokumentation und Bereitstellung von Fachdaten in einer Form verstanden, die dem Anspruch genügt, Daten dauernd und optimal zum Nutzen der gegenwärtigen und künftigen Generationen zur Verfügung zu stellen. Die Daten werden dem Nutzer damit so präsentiert bzw. hinterlassen, dass sie als Entscheidungsgrundlage jederzeit nutzbar sind" (SCHULZE et al. 1999). Die nachhaltigen Datenbestände sollen personenunabhängig, in ein Zeit-Raum-Bedeutungs-Gefüge eingebunden und nach den jeweiligen Zielsetzungen und Fragestellungen kombinierbar sein.

Die Interoperabilität wird als Fähigkeit eines Systems verstanden, Informationen mit anderen Benutzergruppen $\mathrm{zu}$ teilen und Prozesse in Kooperation $\mathrm{zu}$ steuern. Die formalisierten räumlichen Konzepte einer Kommunität und/oder einer Fragestellung sind Ausgangspunkt einer Ontologie.

\section{Konzeptionelle Fragen der Bildung eines Planungsunterstützungssystems}

Mit zunehmender Komplexität der Entscheidungs- und Datenverarbeitungsprozesse kann den vielfältigen Aufgaben der forstlichen Planung nur durch eine effiziente computergestützte Arbeitsweise Rechnung getragen werden. Dabei ist die Gewährleistung der funktionalen Rationalität der Planung, die auf der Beziehung der vorhandenen Informationen und der Qualität der Entscheidung aufgebaut ist, nur ein Teil der verlangten Funktionalität eines solchen Systems. Die kommunikative Rationalität, welche Offenheit, Entschlossenheit, Partizipation, Dialog, Kompromiss und Konsensussuche während des Planungsprozesses voraussetzt, spielt eine immer größere Rolle in der Planung. GIScience trägt hier zur Überbrückung der „,communicate versus calculate“ Dichotomie der Planung bei (NEDOVIC-BUDIC 1999). 


\subsection{GIS Einsatz in der Forstwirtschaft}

Ein Informationssystem ist eine Komposition von Hardware, Software und Daten, welche in ihrer Interaktion mit dem Benutzer Informationen erzeugen und verwalten. Die Informationen als Aussagen, die den Erkenntnisstand eines Subjektes in einem Problembereich erweitern (vgl. ERNI \& LEMM 1998), sind Grundlage von Entscheidungen. Die Aufgabe der Informationssysteme ist es, Informationen $\mathrm{zu}$ strukturieren und zusammenzuführen, ihren Zugriff zu erleichtern, Zusammenhänge und Abhängigkeiten aufzuzeigen, Erklärungsansätze zu unterstützen und Entscheidungsfindung auf eine breitere Grundlage zu stellen (vgl. SPELLMANN 1991).

Die Vorteile des GIS als einer Plattform des betrieblichen Informationssystems, als zentrales Planungsinstrument wurden bereits Anfangs der 90er Jahre entdeckt (STROBL 1988, BITTER 1990, GRIESS 1990): „Die Forsteinrichtung ist ein Geographisches Informationssystem, das betriebsinterne Daten mit Hilfe von Wachstumssimulationen laufend aktuell hält und das gemeinsam mit aktuellen, betriebsexternen Daten ein kybernetisches Rückkoppelungssystem beeinflusst, welches die Grundlage für eine optimale ökologisch-ökonomische Bewirtschaftung eines Forstbetriebes darstellt" (GRIESS 1990). Mittlerweile ist das GIS in vielen Forstbetrieben Standardausstattung geworden.

Die Unterstützung des Planungs- und Produktionsprozesses durch GIS ist vielfältig. „Planners can apply geographic information technologies in all aspects of the planning process, including data collection and storage, data analysis and presentation, planning and/or policy making, communication with the public and decision makers, and planning and/or policy implementation and administration" (NEDOVIC-BUDIC 1999). Während auf der strategischen Ebene sowie in Wissenschaft und Forschung GIS bereits einen Einsatz gefunden hat, werden die Möglichkeiten dieser Technologie auf der operationellen Ebene im täglichen Betriebsgeschehen immer noch eher zögernd genutzt (LÖFFLER 1998).

Nach SCHMIDTKE (1989) und SEKOT (1993) kann ein GIS die Informationssituation des Betriebsleiters durch viele Anwendungsmöglichkeiten positiv beeinflussen:

- Verbesserung der Datenbasis durch Übernahme von Fremddaten

- Verbesserung der Datenverfügbarkeit

- Aktualisierung der Daten durch ständigen Informationsfluss

- Rationalisierung der Kartographie

- Erweiterung der Kartographie

- Gewinnung neuer Information durch Verknüpfung der Daten nach beliebiger Fragestellung

- Entwicklung neuer raumbezogener Inventur-, Kontroll- und Planungsmethoden.

\section{Datenintegration}

Eine wichtige Eigenschaft des GIS, die man sich bis jetzt am meisten zu Nutzen gemacht hat, ist die Fähigkeit, vielfältige Daten durch gemeinsame Referenz zu verknüpfen. Der geographische Raum, der als Referenz dient, spielt dabei eher eine passive Rolle. Seine Aufgabe ist es, die Informationen mit gleichem oder annährend gleichem Bezugsobjekt zu verknüpfen. Das Potential der Integration eines GIS hat in früheren Phasen zu seiner Entwicklung stark beigetragen. Bereits das erste GIS, „Canada Geographic Information System“, sollte für die Automatisierung der 
Informationsverarbeitung sorgen, welche in Kartenform von Canada Land Inventory zusammengestellt wurden (s. Kap. 4.1).

Tab. 19: Anwendungsbeispiele von GIS in der Forstwirtschaft.

\begin{tabular}{|c|c|c|}
\hline Anwendungsbereich & Anwendung & Autor \\
\hline $\begin{array}{l}\text { Datenintegration } \\
\text { und Kartographie }\end{array}$ & $\begin{array}{l}\text { Integrales IS } \\
\text { Kartographie }\end{array}$ & $\begin{array}{l}\text { GRIESS (1990), STROBL (1990), } \\
\text { BITTER (1990) } \\
\text { ROTTMANN \& SCHREYER (1991) }\end{array}$ \\
\hline $\begin{array}{l}\text { Modellierung } \\
\text { und Analyse }\end{array}$ & $\begin{array}{l}\text { typenorientierte Kontrollstichprobe } \\
\text { Kartierung der Bauarteneignung } \\
\text { Erschließungsplanung } \\
\text { Regionalisierung von Klimadaten } \\
\text { Waldzustandsinventur und -analyse } \\
\text { Flächenfragmentierung, Strukturelle Diversität } \\
\text { Fahrspuranalyse } \\
\text { Kartierung und Auswertung von Waldschäden } \\
\text { Abgrenzung von Wuchsräumen }\end{array}$ & $\begin{array}{l}\text { BITTER (1998B) } \\
\text { LEXER et al. (1997) } \\
\text { SHIBA et al. (1990), HENTSCHEL } \\
\text { (1999), PACOLA (1999) } \\
\text { MUES (2000) } \\
\text { FAISST et al. (1998) } \\
\text { TRAUB \& KLEINN (1997) } \\
\text { STEINMAYR et al. (1997) } \\
\text { SIEDE (1991), SCHERRER (1993) } \\
\text { JANSEN et al. (1999) }\end{array}$ \\
\hline $\begin{array}{l}\text { Modellierung und } \\
\text { Entscheidungsfindung }\end{array}$ & $\begin{array}{l}\text { Einsatz der Technologie } \\
\text { Verjüngungsplanung auf Bestandesebene } \\
\text { ökologische Raumbewertung } \\
\text { Umweltverträglichkeit bei der Walderschließung } \\
\text { Holzernteplanung }\end{array}$ & $\begin{array}{l}\text { SPORS et al. (1994) } \\
\text { VACIK (1999) } \\
\text { BÖGEL et al. (1998) } \\
\text { DOUKAS (1997) } \\
\text { LÜTHY (1998) }\end{array}$ \\
\hline
\end{tabular}

In vielen Anwendungsbeispielen spielt die Datenverwaltung und expliziter Raumbezug der betrieblichen Daten eine zentrale Rolle. Die Zusammenführung und Interpretation forstrelevanter Daten war vor dem Einsatz von GIS nur partiell möglich und eine Synopsis schwierig (TRÄNKNER \& SIEDE 1989). Ohne sie ,ist eine moderne Forsteinrichtung noch nicht durchführbar“ (GRIESS 1990).

An die Planungsinformationen werden in einem integrierten GIS in mittleren und größeren Forstbetrieben auch Buchhaltung, Rechnungswesen und Controlling angeknüpft (LÖFFLER 1998). Das betriebliche Forst-Information-System (FIS) der Firma Abies besteht beispielsweise aus den untereinander verknüpften Modulen Holzeinschlag, Faktura, Betriebsbuchführung, Lohn, Dynamisches Betriebswerk, Planung, Auftrag und Jagd (Kessler \& Schöllman 1997). Ein interessantes Beispiel einer Verknüpfung der Daten auch über die Grenze eines Forstbetriebs hinaus stellt das Wald-Ökologische Forstliche Informationssystem (WÖFIS) des Landes Thüringen dar, das die Globalinformationen und Informationen auf Forstamtebene miteinander kombiniert (KOCH \& SCHMALTSCHINSKI 1997).

Eine zweite wichtige Eigenschaft eines GIS ist seine Fähigkeit, durch eine gemeinsame Referenz Informationen $\mathrm{zu}$ verknüpfen und dadurch neue Informationen $\mathrm{zu}$ erzeugen. GIS stellt somit einen Informationsgenerator für raumbezogene Sekundärinformationen dar (SEKOT 1993).

\section{Modellierung und Simulation}

Der Rationalisierungszwang der heutigen Zeit führt zur Änderung der traditionellen Planungen im Wald. So wird beispielsweise gefordert, die physische Präsenz auf der Fläche einer 
Waldeinheit zumindest teilweise durch rechnergenerierte Informationspräsenz zu ersetzen (LÖFFLER 1998). Diese Entwicklung entspricht dem Bemühen, die gemeinsame Referenz der Daten zur Entdeckung und Analyse der Zusammenhänge zu nutzen und somit den Informationsgehalt der bestehenden Datenbestände besser auszuschöpfen.

Ein GIS kann Grundlage für die Herleitung von Wachstumsmodellen (lokalen Standortsertragstafeln) sowie von waldbaulichen Behandlungsmodellen sein. GeoInformationssysteme erscheinen geeignet, als Ergänzung zu Buchführung und Rechnungswesen das IS von Forstbetrieben zu verbessern. Weitere Ansatzmöglichkeiten liegen im Bereich der quantitativen Methoden zur Beschreibung von räumlichem Verteilungsmuster, Struktur, Flächenformen und Fragmentierung (TRAUB \& KLEINN 1997).

Ein GIS bietet mit seiner visuellen Komponente die Möglichkeit, den Informationsinhalt durch wissenschaftliche Visualisierung (visuelle Datenexploration während direkter Interaktion) und visuelle Kommunikation darzustellen.

\section{Entscheidungsunterstützung}

Mit zunehmender Informationsüberflutung reichen die kognitiven Fähigkeiten der Menschen nicht aus, um die Entscheidungsprobleme $\mathrm{zu}$ überschauen und rational $\mathrm{zu}$ entscheiden. Die Äußerung der Präferenzen ist angesichts der Komplexität immer schwieriger und wird von dem Entscheidungsträger verweigert. Der Raumbezug trägt zusätzlich zur Komplexität der Entscheidungssituation bei. Der Raumbezug ist dabei bei den wichtigsten Entscheidungen wie bei dem Aufbau der räumlichen Ordnung, beim Wegebau mit Rücksicht auf die Erschließung und die Holzerntekosten (KOCH \& SCHMALTSCHINSKI 1997) unerlässlich und die Konsequenzen der Alternativen sind von großer Bedeutung. Das entscheidungsrelevante Potential von GIS ist während der gesamten Informationsverarbeitungskette vorhanden: Die Entscheidungsunterstützung durch die Überführung der Daten zu Informationen und weiter zum Wissen hat bereits in früheren Anwendungsbereichen stattgefunden. GIS erwies sich in diesen Fällen als ein geeignetes Tool für Controlling. In dem letzten Anwendungsbereich, der Entscheidungsunterstützung im engeren Sinne, werden auch die Präferenzabbildung eines Entscheidungsträgers und die transparente Lösungsdarstellung unterstützt.

Die Internetfähigkeiten eines GIS (WebGIS), welche die Präsentation von Geoinformationen und den verteilten Zugriff auf die raumbezogenen Daten erlauben, steigern das Potential eines GIS für ein offenes Planungsunterstützungssystem. Wegen seiner Universalität sowie der wachsenden Anzahl der Nutzer wird Web-Access mit dem Public access gleichgesetzt (NedOVIC-BUDIC 1999). 


\subsection{Räumliche Handlungssituation in der Forstwirtschaft}

Dieses Kapitel beschreibt exemplarisch räumliche Handlungssituationen, in welchen Entscheidungsprobleme auftreten. Diese Beispiele beschreiben schematisch die Elemente räumlicher Szene sowie die Alternativen- und Kriterienbildung.

Das Ziel dieser Beispiele ist es, die Formulierung und Anwendung von räumlichen Konzepten zu zeigen, die zur Kriterienbildung und Alternativenbeurteilung herangezogen werden können. Die Beispiele der Handlungssituationen sind so formuliert, dass an ihnen die Anwendung der räumlichen Entscheidungsfindung sowie die Struktur räumlicher Entscheidungsprobleme näher erläutert werden kann. Die Beispiele wurden formuliert, um das Problembewusstsein räumlicher Entscheidungsfindung und das Bewusstsein über ihre Einsatzmöglichkeiten in der Forstwirtschaft $\mathrm{zu}$ stärken. In Tabelle 20 sind die hervorgehobenen Hauptmerkmale der folgenden Entscheidungsprobleme zusammengefasst.

Die Beispiele werden sich auf die Räumlichkeit und ihren Einfluss auf die Entscheidungsfindung beschränken. Die thematischen Eigenschaften der Geoobjekte werden nur dann explizit herangezogen, wenn es für die räumliche Komponente der forstlichen Geoobjekte nötig wird. In den Beispielen dieses Kapitels werden Konzepte vorgestellt, keine konkreten Daten. Eine Lösung von konkreten Entscheidungsproblemen, für welche Geodaten eingesetzt werden können, setzt die Äußerung von Präferenzen eines Entscheidungsträgers voraus. Die gefundene Lösung ist dann die 'optimale' nur für die gegebene Zielsetzung und Präferenzen. Eine Verallgemeinerung solcher Lösungen ist nicht möglich. Aus diesem Grund wurde bei der Formulierung der Beispiele den räumlichen Konzepten größere Bedeutung beigemessen.

Tab. 20: Hervorgehobene Merkmale der räumlichen Entscheidungsprobleme.

\begin{tabular}{l|l}
\multicolumn{1}{c|}{ Entscheidungsprobleme } & \multicolumn{1}{c}{ Merkmale } \\
\hline Technologieeinsatz & räumliches Muster \\
Wahl der Waldentwicklungs-Zieltypen & räumliche Diversität \\
Walderschließung & räumliche Beziehungen eines Graphen \\
Verjüngung & $\begin{array}{l}\text { Komplexe Entscheidungssituation mit } \\
\text { interdependenten Entscheidungen }\end{array}$
\end{tabular}

\subsubsection{Technologieeinsatz}

\subsubsection{Motivation}

Entscheidungen über den Einsatz von Technologie sind wichtige operative Entscheidungen. Mechanisierte Ausführung der Arbeitsprozesse ist in allen Fällen der manuellen vorzuziehen, wo dadurch andere Ziele nicht im erheblichen Maße verletzt werden. Die Entscheidungssituation wird am Beispiel eines Harvestereinsatzes (Kranvollernter) betrachtet, der in den letzten Jahren vor allem wegen der Arbeitssicherheit und Wirtschaftlichkeit weiter zugenommen hat. In niedersächsischen Landesforsten werden bereits rund 50\% des Holzeinschlages mit Vollerntern durchgeführt. Das Design der Handlungssituation ist jedoch auf ein beliebiges Technologiemittel übertragbar. 
Das Handlungsproblem besteht darin, geeignete Flächen für den Einsatz des Harvesters (Einsatzorte) abzugrenzen. Der Entscheidungsträger kann bei dieser Fragestellung verschiedene Ziele verfolgen: Monetär messbare Wirtschaftlichkeit des Einsatzes, Minimierung der Umweltbelastung und der Arbeitsbelastung, etc. Die Ziele stehen u.U. in Konkurrenz zueinander: Die naturschonenden Arbeitsausführungen zeichnen sich oft durch hohe physische Belastung der Waldarbeiter sowie durch hohe Kosten aus. Die mechanisierte Arbeitsausführung ist nicht naturschonend, entlastet jedoch Arbeiter und ist meistens wirtschaftlicher als die motor-manuelle. Die Räumlichkeit kann in dieser Fragestellung den Kriterienbewertungen zugrunde liegen oder als Ausprägung der Entscheidungskriterien dienen.

\subsubsection{Modellierung der räumlichen Situation}

Die Einsatzorte einer Technologie sind flächenhafte Objekte (Polygone eventuell mit Löchern). Für ihre Ausscheidung und Bewertung können Beziehungen zu folgenden räumlichen Objekten von Bedeutung sein:

- Waldeinteilung

- Bodeneinheiten/Standortseinheiten

- Wegenetz

- Geländemodell

Sowohl die Waldeinheiten als auch die Boden-/Standortseinheiten stellen flächenhafte räumliche Objekte dar. Das Wegenetz wird als Zusammensetzung linienartiger Objekte (Graph) modelliert (s. Kap. 8.3.3). Das Gelände kann als Tesselation ${ }^{9}$ modelliert werden.

Tab. 21: Entscheidungsrelevante räumliche Beziehungen bei Technologieeinsatz.

\begin{tabular}{l|l|c}
\multicolumn{1}{c|}{ Geoobjekte/ Felder } & \multicolumn{1}{|c}{ geometrische Beziehungsart } & Beziehungsarten \\
\hline Waldeinheiten, & Polygon / Polygon-Beziehungen & Topologie \\
Standortseinheiten & Polygon / Linie (Graph)-Beziehungen & Distanz, Topologie \\
Wegenetz & Polygon / Zelle (Tesselation)-Beziehungen & Topologie \\
Geländeform &
\end{tabular}

Räumliche Beziehungen, welche für die Entscheidung relevant sein können, sind in Tabelle 21 aufgelistet: Als topologische Beziehungen zwischen dem Einsatzort und dem Bestand/Standort kommen insgesamt 6 Fälle in Betracht, welche mit dem topologischen Modell der Inhaltsinvariante (s. Kap. 5.3.2.1) unterschieden werden können (Abbildung 57).

Detailliertere topologische Invarianten (z.B. Komponenteninvarianten) können mögliche Beziehungen weiter verfeinern. Die metrischen Konzepte, mit welchen die Beziehungen

${ }^{9}$ Eine Tesselation kann z.B. wie folgt definiert werden:

$S$ sei eine abgeschlossene Untermenge von $\mathfrak{R}^{\mathrm{d}}, \mathfrak{I}=\left\{s_{1}, \ldots s_{n}\right\}$, wobei $s_{i}$ eine abgeschlossene Untermenge von $\mathrm{S}$ ist. $\mathrm{s}_{\mathrm{i}}{ }^{\circ}$ ist das Innere von $\mathrm{s}_{\mathrm{i}}$. $\mathfrak{I}$ wird Tesselation genannt, wenn ihre Elemente folgende Bedingungen erfüllen:

$\mathrm{s}_{\mathrm{i}}{ }^{\circ} \cap \mathrm{s}_{\mathrm{j}}{ }^{\circ}=\varnothing$ für $\mathrm{i} \neq \mathrm{j}$

$\bigcup_{i=1}^{n} s_{i}=S$ 
quantifiziert werden können (Kap. 5.3.2.8), sorgen ebenfalls für eine detailliertere Auflösung der Beziehungen.

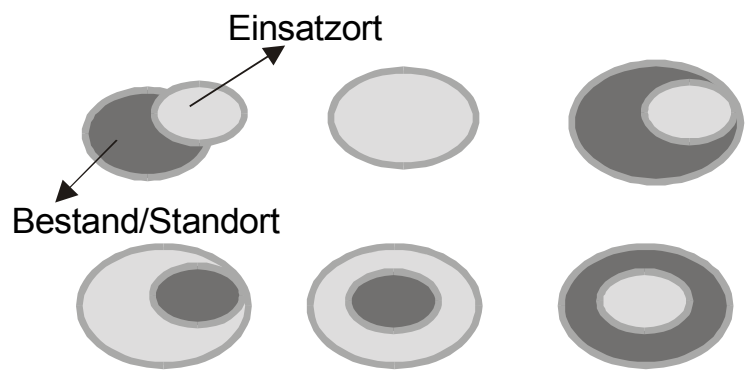

Abb. 57: Mögliche topologische Beziehungen der Inhaltsinvariante zwischen den geeigneten Einsatzorten und dem Bestand/Standort.

Die Beziehungen zwischen den potentiellen Einsatzorten und dem Wegenetz bzw. der Transportgrenze hängen davon $\mathrm{ab}$, ob diese aus der konzeptionellen Sicht als Objekte mit oder ohne Rand modelliert werden (s. auch Kap. 8.3.3). In dem ersten Fall, wenn der Rand des Wegenetzes für die Fragestellung nicht von Bedeutung ist, erlaubt die Inhaltsinvariante nur 3 unterschiedliche Fälle (Abbildung 58:1-3), während in dem zweiten Fall zusätzlich 3 Beziehungen möglich sind (Abbildung 58:4-6).

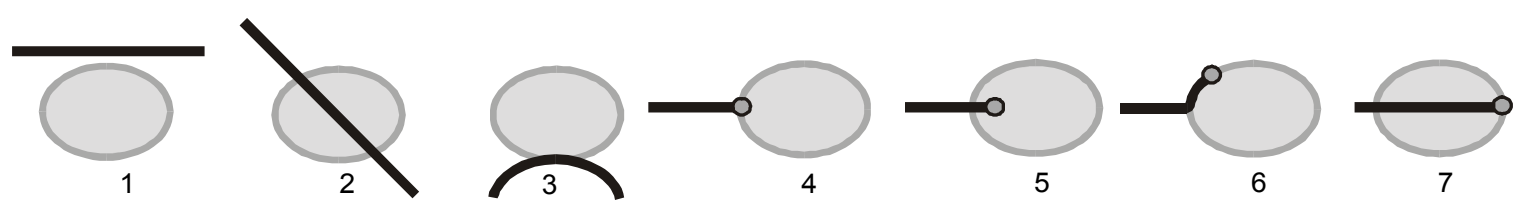

Abb. 58: Mögliche topologische Beziehungen der Inhaltsinvariante zwischen den Wegen und polygonhaften räumlichen Objekten: 1-3) ohne Rand 4-6) zusätzliche Beziehungen für Wege mit Rand.

Abhängig von der räumlichen Datenstruktur des Geländemodells können die Geländeelemente von Raster- oder von TIN ${ }^{10}$-Zellen gebildet werden. In beiden Fällen ist die Neigung und Exposition der Zellen für ihre ganze Ausdehnung konstant. Zugängliche Flächen werden als Komposition der Zellen mit entsprechender Hangneigung gebildet. Jede zusammenhängende zugängliche Fläche wird als ein Kompositionsobjekt gebildet. Der räumliche Zusammenhang des Kompositionsobjektes (topologische Beziehung meet) kann mit der Dimension des gemeinsamen Randes eingeschränkt werden. Dies kann die Beziehungen der Einsatzorte zueinander beeinflussen: zwei für den Einsatz geeignete Flächen, die in einem Punkt oder an einer Linie kleiner Länge aneinander grenzen, können zu zwei unterschiedlichen Kompositionsobjekten gehören.

Die Kompositionsobjekte können Löcher enthalten. In diesem Fall schließt das Kompositionsobjekt eine für den Einsatz ungeeignete Fläche in seinem Inneren ein. Diese Fälle sind graphisch in Abbildung 59 beschrieben. Umgekehrt ist es möglich, dass eine geeignete Fläche von einer unzugänglichen umschlossen wird und sie daher unerreichbar wird.

${ }^{10}$ TIN - Triangulated irregular network, dreieckförmige Raumzerlegung geeignet für die Modellierung kontinuierlicher Phänomene 
A)

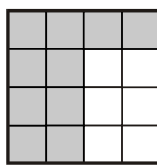

B)

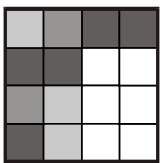

1)
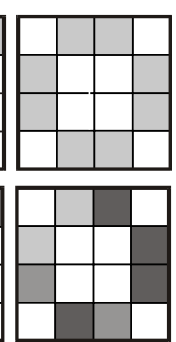

2)

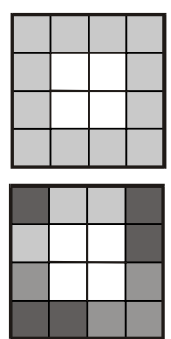

3)
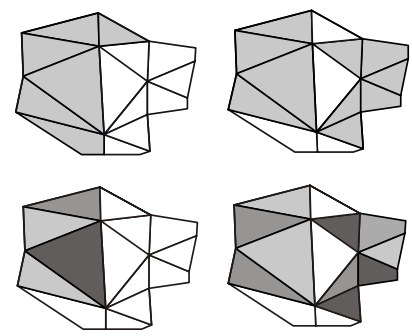

4)

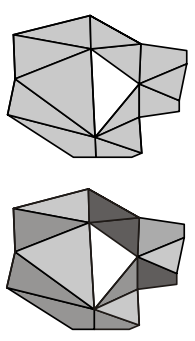

6)

Abb. 59: Komposition von Zellen der Geländerepräsentation: (1-3) Rasterrepräsentation, (4-6) TIN Repräsentation; A) zweiwertige Bewertung, B) mehrwertige Bewertung; 1,4) 1D meet zusammenhängende Zellen; 2,5) 0D meet zusammenhängende Zellen; 3,6) eingeschlossene Fläche.

Als ein weiteres räumliches Konzept in diesem Beispiel wird die räumliche Verteilung (räumliches Muster) der geeigneten Einsatzorte erläutert. Es ist vorstellbar, dass aufgrund eines stark gegliederten Reliefs die geeigneten Einsatzorte klein sind und weit voneinander zerstreut liegen. Dies würde den Einsatz der mechanisierten Arbeitsausführung gegenüber der manuellen in bezug auf andere Ziele (Wirtschaftlichkeit des Einsatzes, Umweltschonung) benachteiligen. In Abbildung 60 ist das Konzept des räumlichen Musters schematisch dargestellt: die Flächen mit unterschiedlicher Eignung für den Technologieeinsatz liegen unregelmäßig verteilt (a) oder konzentriert in Bereichen mit gleicher Eignung (Cluster) (b) oder konzentriert in einem Bereich.

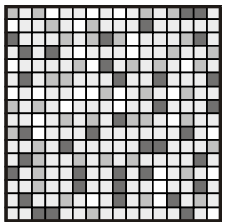

a)

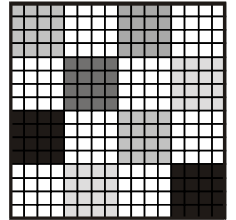

b)

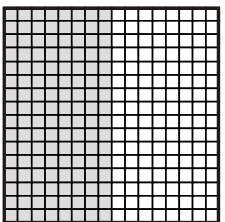

c)

Abb. 60: Konzept des räumlichen Musters: a) unregelmäßige Verteilung nicht zusammenhängenden Eignungsflächen; b) Verteilung der zusammenhängenden Bereiche (Cluster) mit gleicher Eignung; c) zusammenhängende 2-wertige Eignung für den Einsatz.

Die räumlichen Beziehungsarten können unterschiedliche Eigenschaften des Verteilungsmusters beschreiben:

- Topologische Beziehungen betreffen die Aggregation einzelner Einsatzorte im Cluster, sie beschreiben seine innere Struktur (Abbildung 61a).

- Distanzbeziehungen drücken räumliche Ausdehnung des Clusters aus und daher seine Größe (Abbildung 61b).

- Richtungsbeziehungen beschreiben die Ausrichtung und Form des Clusters (Abbildung 61c).

Jede Beziehungsart beschreibt eine 'Dimension' der Musterbeschreibung. Zu einer integralen Musterbeschreibung müssen diese geeignet aggregiert werden. Beziehungstabellen ${ }^{11}$ (Abbildung 62) stellen einen Ausgangspunkt der Aggregation dar.

\footnotetext{
${ }^{11}$ Quadratische Tabelle paarweiser Beziehungen von Objekten einer räumlichen Szene.
} 


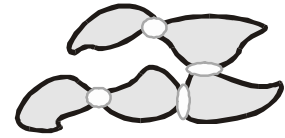

a)

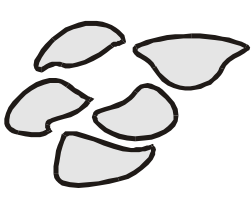

b)
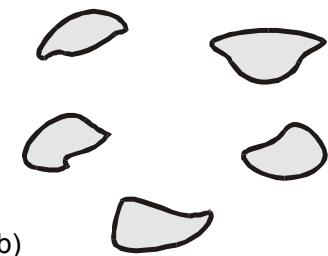

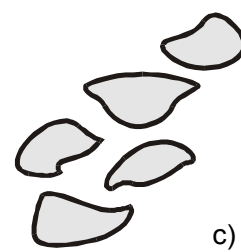

c)

Abb. 61: Dimensionen der Musterbeschreibung: a) Aggregation; b) Größe; c) Form und Ausrichtung.

In der Point Pattern Analysis ${ }^{12}$ wird beispielsweise ein nearest neighbor index als Verhältnis der empirischen und erwarteten durchschnittlichen Entfernung zum nächsten Nachbarn als ein Maß zur Beschreibung des räumlichen Musters angewendet. Die Situation bei den Punkten ist jedoch durch fehlende räumliche Ausdehnung vereinfacht.

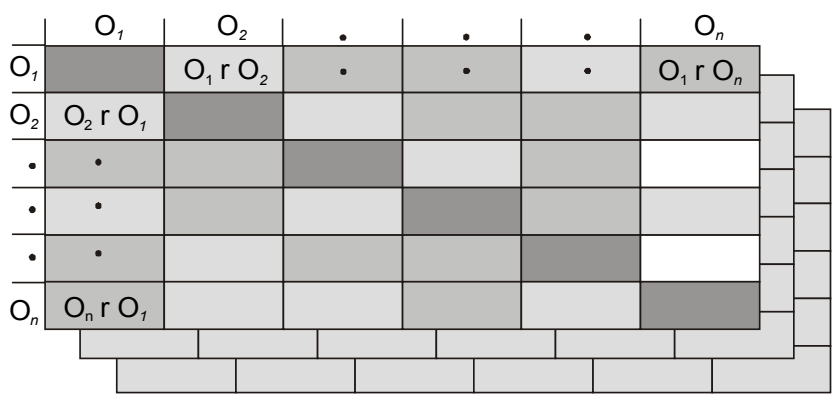

Abb. 62: Beziehungstabellen einzelner ,Dimensionen’ der Musterbeschreibung.

\subsubsection{Entscheidungsfindung}

Bei der Fragestellung über den Technologieeinsatz kann die gesuchte Entscheidung als Zuordnung einer Arbeitsausführung einem Ort oder als Abgrenzung des Einsatzortes für eine Arbeitsausführung (Technologie) betrachtet werden. Die Lösungssuche kann in zwei Schritten formuliert werden, indem im ersten Schritt harte, nicht kompensierbare Entscheidungskriterien (constraint) zur Ausscheidung der potentiellen Einsatzorte angewendet werden und diese im zweiten Schritt einer Entscheidung über den Einsatz unterzogen werden.

Der Technologieeinsatz wird vor allem durch die Geländezugänglichkeit und Bodenbelastbarkeit, sowie durch die Bestockungseigenschaften bestimmt. Beispielsweise für den Einsatz eines Harvesters galten in SPORS et al. (1992) Flächen mit einer Hangneigung kleiner 30\% als „zugänglich“, als ,geeignet" gering bis mäßig gefährdete Standorte (abgeleitet nach den bodenökologischen Eigenschaften: Wasserhaushalt und geologisches Substrat) und als ,geeignet“" nach Bestandeseigenschaften Nadelholzrein- und -mischbestände ab zweiter Durchforstung im Durchmesserbereich 12 bis $35 \mathrm{~cm}$.

Diese Kriterien können als nicht kompensierbar (constraints) angenommen werden. Wird diese Fragestellung mit Kompensationsmöglichkeit einer schlechten Ausprägung bezüglich eines Kriteriums mit einer besseren Ausprägung bezüglich eines anderen Entscheidungskriteriums zugelassen, so bekommt die Fragestellung einen größeren Spielraum für die gesuchte Entscheidung. Die Hangneigungen zwischen 20-30\% können beispielsweise als „bedingt

12 Point Pattern ist ein räumliches Muster der Verteilung einer Punktmenge. Bei seiner Analyse sind die räumlichen Eigenschaften der gesamten Punktmenge von Bedeutung. 
geeignet“ angenommen werden, ebenfalls mäßig gefährdete Standorte und Nadelholzmischbestände.

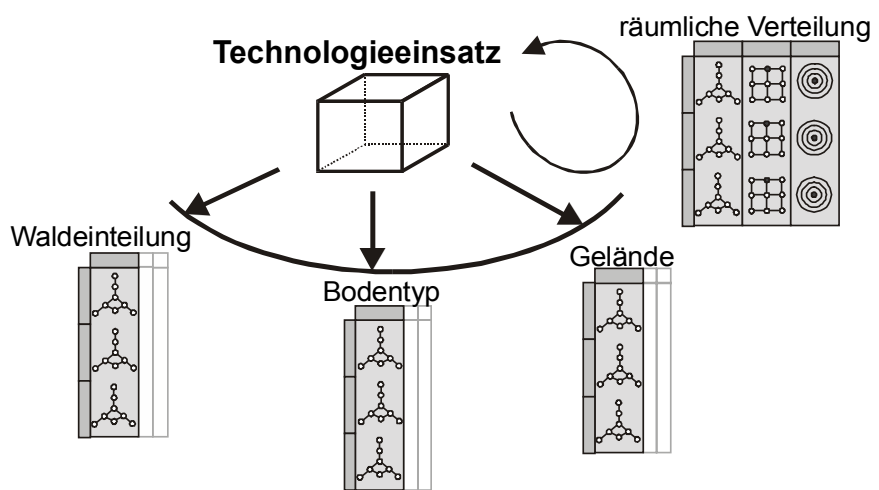

Abb. 63: Räumliche Konzepte der Handlungssituation für den Technologieeinsatz.

Als weitere mögliche Kriterien können beispielsweise der Anteil des potentiellen Einsatzortes an der Bestandesfläche, Transportmöglichkeit des technologischen Mittels in den Bestand/Einsatzort, etc. angenommen werden. Der Entscheidungsträger kann eine möglichst einheitliche Durchführung der geplanten Maßnahmen in einem Bestand anstreben. Er ist aber bereit, sollte es möglich sein, auf relativ großem Anteil der Bestandesfläche die Technologie einzusetzen, diese der manuellen Arbeitsausführung vorzuziehen. Die Verteilung der potentiellen Einsatzorte im Bestand kann ebenfalls von Bedeutung sein. Kleine Flächen, die im Bestand weit voneinander liegen, können als ungeeignet angesehen werden. Die potentiellen Einsatzorte können nach ihrer Zugänglichkeit mit dem Kriterium "Abstand vom Wegenetz" bewertet werden. Um große bestandesübergreifende Zersplitterung der Einsatzorte $\mathrm{zu}$ vermeiden, wird der Harvester nur dort eingesetzt, wo eine entsprechend große Konzentration der Einsatzorte vorliegt.

Die bevorstehende Entscheidung setzt die Beurteilung der Zielerfüllung für jede konkrete räumliche Situation eines oder mehrerer potentiellen Einsatzorte voraus. Räumliche Konzepte dienen in diesem Beispiel als Strukturierungskonzepte der Zielerreichung. Für den Einsatz der Technologie wird überall dort entschieden, (i) wo die eigenen Eigenschaften eines potentiellen Einsatzortes (Größe usw.) und (ii) wo die räumliche Anordnung der potentiellen Einsatzorte günstig sind.
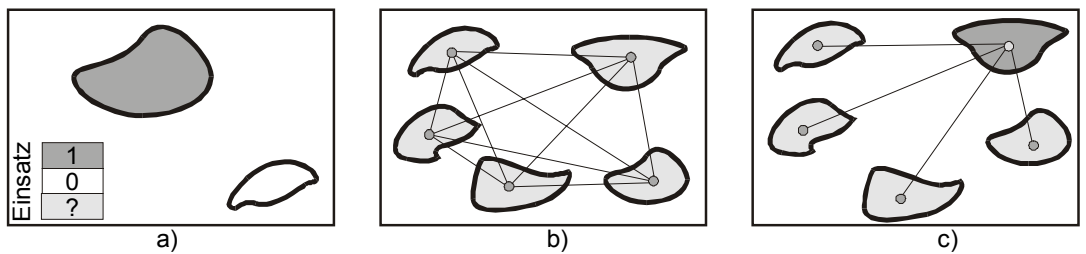

Abb. 64: Mögliche Designs der Entscheidungssituation: a) individuelle Entscheidungen anhand eigener Eigenschaften potentieller Einsatzorte, b) Entscheidungen anhand räumlicher Konfiguration von potentiellen Einsatzorten, c) Entscheidungen anhand der räumlichen Beziehungen zu bereits als vorteilhaft klassifizierten Einsatzorten.

In Abbildung 64 sind diese Situationen schematisch dargestellt: in (a) kann für den Einsatz unabhängig von der räumlichen Konfiguration entschieden werden, in (b) wird anhand der 
räumlichen Anordnung entschieden und in (c) wird anhand der räumlichen Beziehungen zu den bereits als vorteilhaft klassifizierten Einsatzorten entschieden.

\subsubsection{Wahl der Waldentwicklungszieltypen}

\subsubsection{Motivation}

Das Regierungsprogramm zur langfristigen, ökologischen Waldentwicklung (LÖWE) verpflichtet, die Grundsätze eines naturnahen Waldbaus auf ökologischer Grundlage als bindende Handlungsanweisung für die Gestaltung der Landesforsten zu berücksichtigen. Diese Grundsätze werden in Form des angestrebten Waldzustandes formuliert, dessen Leitbild durch die Waldentwicklungstypen beschrieben wird.

Je nach Standort werden für jede Waldeinteilungseinheit bis $\mathrm{zu} 5$ alternative Waldentwicklungstypen empfohlen, die nach der Standorteignung geordnet sind. Neben dem Ziel der Standorteignung kann der Entscheidungsträger auch noch weitere Ziele anstreben, in welchen die Räumlichkeit enthalten sein kann. Beispielsweise kann er an der räumlichen Diversität der flächenbezogenen Waldeinheiten in bezug auf den Bestandestyp (Baumartenzusammensetzung) interessiert sein. Das Ziel der bevorstehenden Entscheidung ist demnach eine bestmögliche Zuordnung der Waldentwicklungstypen zu den Waldeinheiten in bezug auf das Ziel der Standorteignung und der räumlichen Diversität.

\subsubsection{Modellierung der räumlichen Situation}

Die räumliche Diversität stellt das entscheidungsrelevante räumliche Konzept der Entscheidungssituation dar. Die Diversität ist ein Maß der Variabilität in nominalen Merkmalen. Sie ist das Gegenstück der Varianz metrisch skalierter Merkmale. Ein Diversitätsmaß, das sowohl die Anzahl unterschiedlicher Arten (Objekte oder Phänomene mit bestimmten Eigenschaften) $(s)$ als auch ihre Häufigkeit $\left(p_{i}\right)$ berücksichtigt, soll bestimmten Anforderungen genügen (PIELOU 1975, zitiert nach BACKOWSKI et al. 1997):

- für eine bestimmte Ausprägung von $s$ gilt, das Diversitätsmaß soll sein Maximum dann erreichen, wenn alle $p_{i}$ gleich sind

- wenn alle $p_{i}$ gleich sind, soll das Maß mit steigendem $s$ wachsen.

In der Literatur kann eine Vielzahl an Indizes gefunden werden (SHANNON, SIMPSON, GoOD zitiert nach BACKOWSKI et al. 1997), welche Diversität beschreiben.

Für die räumliche Diversität sollen hier nicht nur die Anzahl der Arten und ihre Häufigkeit, sondern auch die Anzahl unterschiedlicher räumlicher Beziehungen $\left(s_{r i}\right)$ und ihre quantitativen Eigenschaften $\left(q_{r i}\right)$ von Bedeutung sein. Für ein räumliches Diversitätsmaß können dann folgende zusätzliche Bedingungen definiert werden:

- Für eine bestimmte Ausprägung von $s_{r i}$, soll das Diversitätsmaß sein Maximum dann erreichen, wenn alle $q_{r i}$ gleich sind, und

- wenn alle $q_{r i}$ gleich sind, soll das Maß mit steigendem $s_{r i}$ wachsen.

Für ein räumliches Diversitätsmaß müssen räumliche Beziehungsarten sowie Konzepte ihrer Quantifikation gewählt werden, welche für die Fragestellung von Bedeutung sind. Für die lokalen Diversitätsmaße muss auch ein Raumausschnitt relativ am einem Zentrum definiert werden (Umgebung), von welchem aus man die räumliche Diversität beschreibt. Im einfachsten Fall wird 
die ganze Umgebung als eine einzige Beziehung definiert. Diese kann als topologische oder distanzbasierte Nachbarschaft definiert werden: Im ersten Fall werden alle räumliche Objekte betrachtet, deren Beziehung zu dem zentralen räumlichen Objekt "meet" ist. Im zweiten Fall bilden die Nachbarschaft alle Objekte, die eine nicht leere Schnittmenge mit der Akzeptanzfläche einer gewählten Distanzbeziehung haben (s. Kap. 5.3.3).

Die räumliche Diversität bezogen auf den Bestandestyp ist ein Maß, das die lokale räumliche Verteilung der Waldeinheiten mit von der zentralen Waldeinheit unterschiedlicher Baumartenzusammensetzung beschreibt. Als dem Diversitätsmaß zugrundeliegende räumliche Konzepte können topologische und metrische (Distanz, Richtung) Beziehungen angenommen werden. Die räumlichen Beziehungen werden in dem Kriterium ,räumliche Diversität‘ aggregiert.

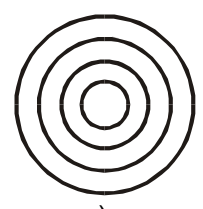

a)

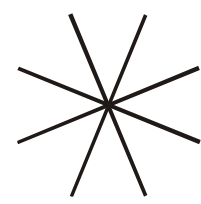

b)

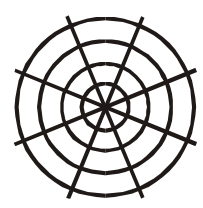

c)

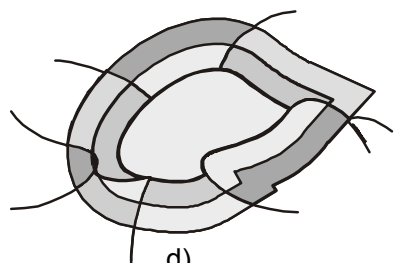

d)

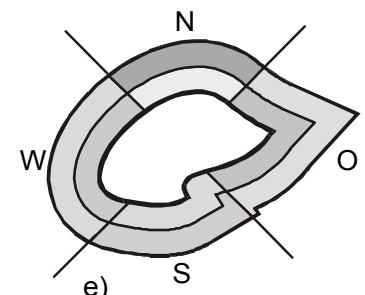

Abb. 65: Räumliche Konzepte und lokale Umgebung: a-c) distanz- und richtungsbasierte Nachbarschaft und ihre Kombination; d) kombinierte topologische und distanzbasierte Nachbarschaft; e) kombinierte topologische und richtungsbasierte Nachbarschaft.

In Abbildung 65 sind schematisch räumliche Nachbarschaftskonzepte sowie unterlegene Konzepte dargestellt. Die unterschiedlichen Beziehungsarten können unterschiedliches Gewicht bei ihrer Aggregation erhalten.

\subsubsection{Entscheidungsfindung}

Die Entscheidungssituation lässt sich je nach der Fragestellung und den aufgetretenen Interdependenzen mit drei Designs beschreiben (s. Abbildung 66).

Im ersten Design soll einer Waldeinheit in Nutzung ein solcher alternativer Waldentwicklungszieltyp (WET) zugeordnet werden, welcher bezüglich beider Ziele (Standortseignung und Diversität) den Präferenzen des Entscheidungsträgers am besten entspricht. In diesem Design tritt nur Konkurrenz zwischen der Standorteignung und dem Beitrag zur Diversität der Waldentwicklungszieltypen auf. 


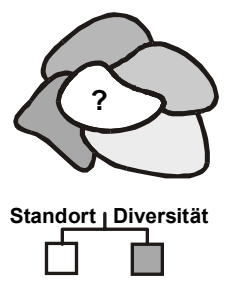

a)

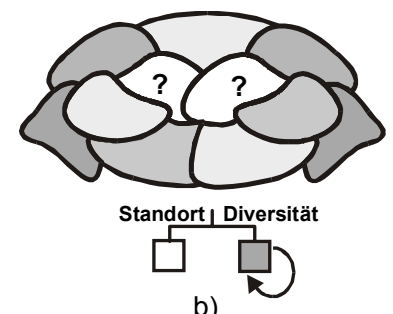

b)

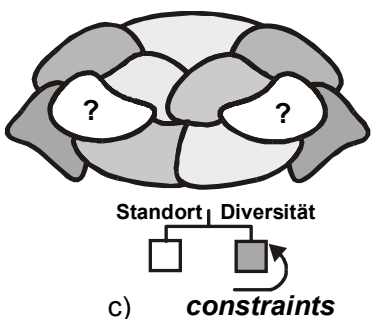

c) constraints

Abb. 66: Mögliche Interdependenzen in der Entscheidungssituation: a) Konkurrenz eines räumlichen und eines a-räumlichen Kriteriums, b-c) zusätzliche Konkurrenz zweier räumlicher Kriterien.

Im Design 2 soll eine Entscheidung für zwei benachbarte Waldeinheiten getroffen werden, deren Zielerreichungen bezüglich der räumlichen Diversität aufgrund der Nachbarschaft beider Waldeinheiten gegenseitig konkurrieren. Die Entscheidung für eine Waldeinheit beeinflusst somit die Entscheidung der zweiten Waldeinheit.

Im Design 3 kommt es zu einer Konkurrenz zwischen räumlich entfernten Waldeinheiten durch eine zusätzliche Einschränkung der angestrebten Anteile von Baumarten. Um eine bestimmte Baumartenzusammensetzung einer Betriebsklasse $\mathrm{zu}$ erreichen, müssen auch für den Standort weniger geeignete Waldentwicklungstypen (WET) angenommen werden. Dabei soll auch räumliche Diversität bei der Verteilung von WET's berücksichtigt werden.
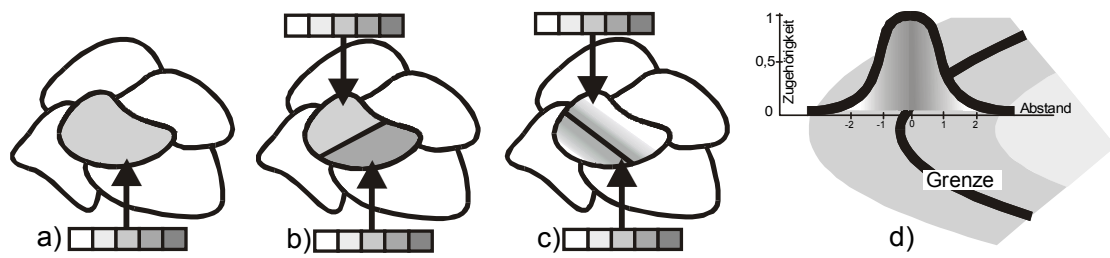

Abb. 67: Design 1 der Entscheidungssituation und seine möglichen Modifikationen: a) einheitlicher Standortstyp auf ganzer Fläche der Waldeinheit; b) zwei Standortstypen in der Waldeinheit; c) räumlich unsicherer Rand der Standortseinheit; d) fuzzy-basierte Darstellung räumlicher Unsicherheit.

In Abbildung 67 sind mögliche Fälle des Design 1 schematisch dargestellt. Der Fall (a) stellt eine einfache Situation dar, in welcher jeder Waldeinheit nur ein bestimmter Standorttyp (und somit nur bis 5 alternativen WET's) entspricht. In dem Fall (b) stockt der Bestand auf zwei unterschiedlichen Standorten, somit entstehen (bei der Erhaltung seiner Grenze) bis zu 2 x 5 alternative WET's, jeweils 5 für jeden Standorttyp. In dem Fall (c) ist die Grenze beider Standortstypen räumlich unsicher, möglicherweise stockt der Bestand auch nur auf einem der Standorte.

Abbildung 68a-b stellt das Design 2 der Fragestellung schematisch dar. Mindestens zwei (höchstens allen) Waldeinheiten soll ein WET zugeordnet werden, wobei zwischen diesen Konkurrenz besteht. Die Abbildung 68c beschreibt den letzten Fall, in dem es zur Konkurrenz aufgrund einer Nebenbedingung kommt. 


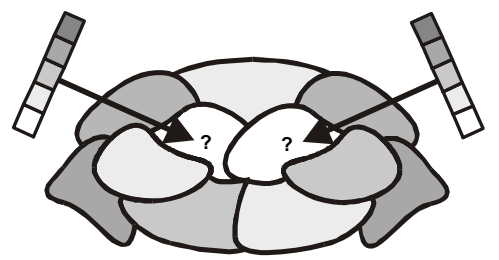

a)

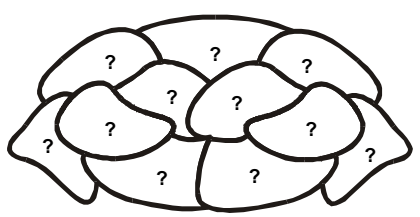

b)

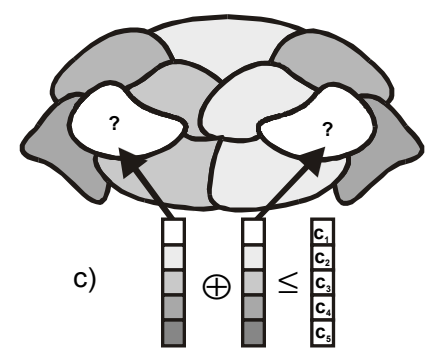

Abb. 68: Design 2 (a-b) und 3 (c) der Entscheidungssituation: a) zwei räumlich-benachbarte Entscheidungssituationen; b) Vielzahl räumlich-benachbarter Entscheidungssituationenen; c) zwei konkurrierende, räumlich nicht zusammenhängende Entscheidungssituationenen.

In allen diesen Handlungssituationen stellt die Räumlichkeit ein Entscheidungskriterium dar. In jedem Design der Entscheidungssituation müssen Präferenzen bezüglich der räumlichen Szene geäußert werden und diese mit Präferenzen bezüglich des a-räumlichen Kriteriums (Standortseignung) aggregiert werden: Die bevorstehende Entscheidung betrifft die Bereitschaft des Entscheidungsträger, einen WET mit schlechterer Standortseignung anzunehmen, um eine bessere räumliche Diversität für eine Waldeinheit zu erzielen. Die Entscheidungsfindung kann durch räumliche Unsicherheit zusätzlich erschwert werden.

\subsubsection{Walderschließung durch Wege}

\subsubsection{Motivation}

Die Walderschließung bildet zusammen mit der Waldeinteilung die horizontale Gliederung des Waldes. Sie ist eine unerlässliche Voraussetzung der forstlichen Produktion, indem sie den Wald zugänglich macht und gleichzeitig Ausgangspunkt zur Erfüllung anderer Aufgaben ist. Sie ist zudem Voraussetzung für nachhaltige Bewirtschaftung, setzt aber auch die Kräfte der Zerstörung natürlicher Lebensräume (Erosion, Exploitation) in Bewegung. Der Waldwegebau kann schließlich auch bleibende Schäden in der Landschaft verursachen (DIETZ et al. 1984).

Die Walderschließung ist ein hierarchisch aufgebautes Gebilde. Der Wald wird zuerst an das öffentliche Verkehrsnetz angeschlossen, dann werden die einzelnen Waldteile durch Verkehrsanlagen im Wald verbunden und schließlich die einzelnen Teilflächen an die Hauptverkehrslinien im Wald angeschlossen. Die Walderschließung ist eine Flächenerschließung, da die flächenbezogenen Wirtschaftseinheiten zugänglich gemacht werden sollen.

Die optimale Lösung der Erschließung kann nicht als statisch angesehen werden. Die wichtigen Kriterien der Lösungssuche unterliegen einer zeitlichen Änderung. Daher ist in der Regel keine über längere Zeiträume konstante optimale Lösung möglich. Entsprechend des hierarchischen Aufbaus wird die Walderschließung in Basiserschließung durch die Fahrwege und Feinerschließung durch die Rückewege gegliedert. Beide Wegearten unterscheiden sich von einander in bezug auf Konstruktion, Zugänglichkeit und Art der Fahrzeuge, für die sie bestimmt sind. 
Tab. 22: Konzepte der Walderschließung.

Wegedichte
Wegeabstand
Erschließungsprozent
Erschließungsbandbreite
Rückeentfernung
Mehrfacherschließung

Zur Entscheidungsfindung in der Walderschließung wurden unterschiedliche Bewertungskalküle entwickelt (DIETZ et al. 1984). Bei diesen wird die Vorteilhaftigkeit der Erschließungsvarianten vorwiegend an denjenigen Konzepten gemessen, die monetär meßbar sind. Methoden der Investitionsrechnung stellen die am häufigsten angewendeten Entscheidungsmodelle bei monetär meßbaren Entscheidungskriterien dar. Andere Methoden wie Kosten-Wirksamkeits-Analyse (KWA) und Nutzwertanalyse (DIETZ et al. 1984) erlauben die Berücksichtigung auch anderer, nicht monetär meßbarer Zielkriterien. Beispiele räumlicher Konzepte, welche zur Beurteilung der alternativen Varianten der Walderschließung angewendet werden, können Tabelle 22 entnommen werden.

\subsubsection{Modellierung der räumlichen Situation}

Ein Wegenetz kann als ein Graph modelliert werden. Ein Graph ist ein Tripel G = (V, E, I), wobei $\mathrm{V}$ eine nichtleere Knotenmenge und $\mathrm{E}$ eine Kantenmenge ist. Eine Inzidenzabbildung I ordnet jeder Kante ein Knotenpaar aus V zu. Eine nicht-leere und endliche Liste von paarweise adjazenten Kanten heißt ein Weg. Je nach den Eigenschaften eines Graphen werden verschiedene Typen unterschieden: Ein zusammenhängender Graph ist ein Graph, in dem jedes Knotenpaar durch mindestens einen Weg verbunden ist. Ein zusammenhängender schleifenloser Graph heißt Baum.
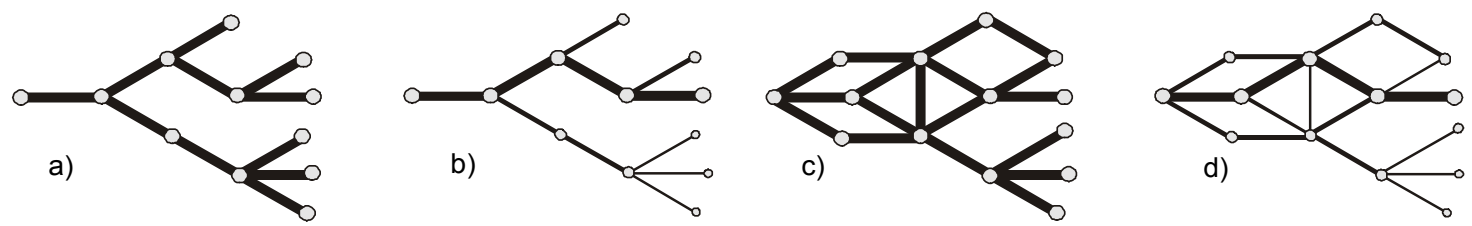

Abb. 69: Wegenetz als Graph: a) spezieller Typ des Graphen, Baum, als einfaches Geoobjekt; b) ein Baum als zusammengesetztes hierarchisch aufgebautes Geoobjekt; c) einfacher Graph; d) zusammengesetzter hierarchisch aufgebauter Graph.

Ein Wegenetz kann aus konzeptioneller Sicht als ein einfacher (Abbildung 69a,c) oder als ein hierarchisch aufgebauter Graph modelliert werden (Abbildung 69b,d). Ein spezieller Typ des Graphen, ein Baum (Abbildung 69a,b), kann die Beschaffenheit des Wegenetzes in manchen Situationen gut beschreiben.

Aus der Sicht der konzeptionellen Modellierung tauchen Fragen auf, die nur in konkreter Fragestellung von dem Entscheidungsträger beantwortet werden können. So sind beispielsweise Fragen $\mathrm{zu}$ beantworten, welche die Bedeutung der Schnittmenge eines Weges und eines Bestandes betreffen: 
- Sind Fälle mit unterschiedlichen topologischen Beziehungen eines Weges zu einem Bestand in Bezug auf die Erschließungsgüte zu unterscheiden?

- Sind die Kanten eines Graphen zu unterscheiden, oder werden alle als gleich ansehen und wird somit nur ein netzwerkbasiertes Objekt modelliert?

- Beeinflussen die quantitativen Eigenschaften oder die genauere Art der Beziehung die Präferenz des Entscheidungsträgers?

- Welche Bedeutung ist der Form des Weges zuzuschreiben, solange sie nicht die Beziehungen beeinflusst?

- Wie kann die Zugänglichkeit eines Bestandes mit Hilfe räumlicher Beziehungen beschrieben werden?

Da ein Graph ein zusammengesetztes Objekt darstellt, treten in dieser Fragestellung 2 unterschiedliche Beziehungen auf:

- Beziehungen der Kanten/des Graphen zu räumlichen Objekten

- Beziehungen der Kanten zueinander

Die erste Art beschreibt die relative Position räumlicher Objekte (Teilflächen, Standorte, Geländeform usw.) zu dem Wegenetz, während die zweite sich mit der Zusammensetzung des Graphen bzw. des Wegenetzes befasst.

\section{Beziehungen zu räumlichen Objekten}

Die räumlichen Beziehungen eines Graphen werden von den Beziehungen seiner Kanten abgeleitet. Die möglichen topologischen Beziehungen eines Graphen (G) hängen vom Typ des Graphen ab. Der Rand eines Graphen $(\partial \mathrm{G})$ wird von den Knoten gebildet, die nur zu einer Kante inzident sind. Ein Graph kann demzufolge auch keinen Rand besitzen, wenn jeder Knoten zu mindestens zwei Kanten inzident ist. In einem Baum wird der Rand von den Endknoten eines Blattes (Knoten ohne Nachfolger) und von der Wurzel (Knoten ohne Vorgänger) gebildet. Hat ein Graph keinen Rand, so können nur solche topologische Beziehungen abgeleitet werden, die von dem Inhalt seiner Schnittmengen unabhängig sind (Abbildung 70).

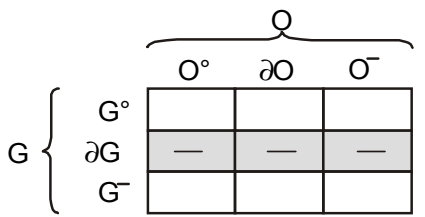

a)

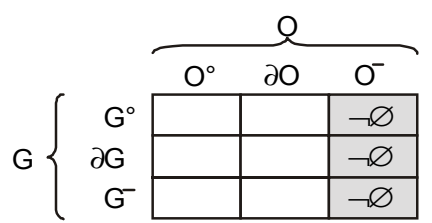

b)

Abb. 70: Einschränkung der Einträge der Intersectionmatrix (Kap. 5.3.2.1.) eines Graphen: a) Graph ohne Rand; b) nach der Bedingung der Größenordnung.

Die zweite Eigenschaft der räumlichen Szene, welche die möglichen Beziehungen beeinflussen kann, ist die Größe der räumlichen Objekte. Sind die räumlichen Objekte (viel) kleiner als die vom gesamten Wegenetz eingeschlossene Fläche, so kann im weiteren angenommen werden, dass die Schnittmenge des Äußeren der Objekte und des Randes, des Inneren und des Äußeren eines Graphen immer nichtleer ist (Abbildung 70). 


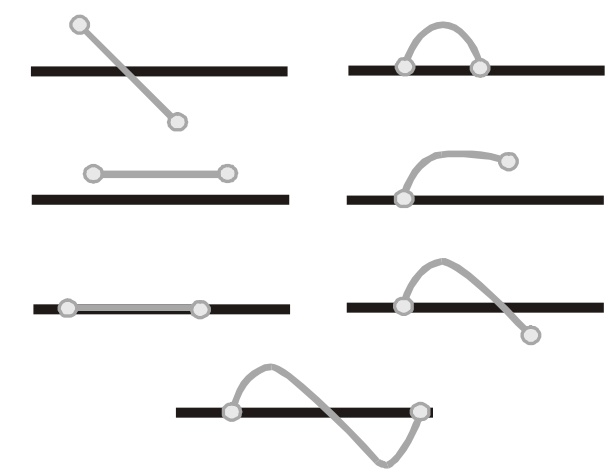

A)

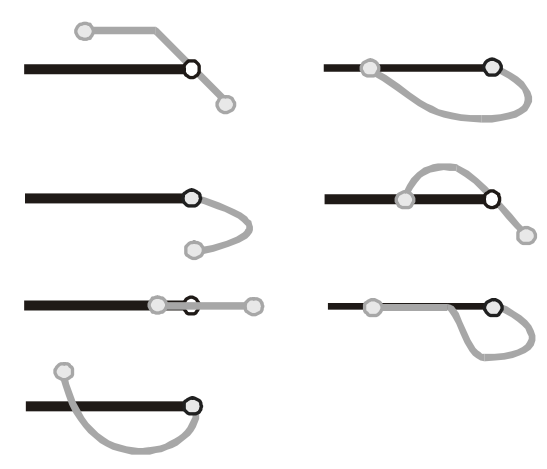

B)

Abb. 71: Beziehungen eines Pfades mit linienartigen räumlichen Objekten: A) mit der in der Abb. 70 definierten Einschränkung; B) zusätzliche Beziehungen im Fall ohne Einschränkung.

Besitzt ein Graph keinen Rand, so können mit Inhaltsinvariante nur 7 Fälle unterschieden werden (Abbildung 71a). Ein Graph mit Rand hat 14 mögliche Beziehungen (Abbildung 71a-b).

Tab. 23: Anzahl der möglichen topologischen Beziehungen eines Graphen nach der leer-nichtleeren Beschreibung der Schnittmengen.

\begin{tabular}{c|c|c} 
räumliches Objekt & Graph ohne Rand & Graph mit Rand \\
\hline Polygon & 3 & 7 \\
\hline Linie & 7 & 14
\end{tabular}

Bei den Beziehungen eines Graphen zu flächenhaften Objekten können je nach dem Typ des Graphen 3 bzw. 7 Fälle vorkommen. Die möglichen Beziehungen der Inhaltinvariante werden in Tabelle 24 und Abbildung 72 beschrieben.

a)

b)

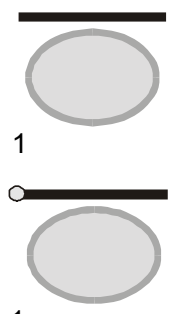

1

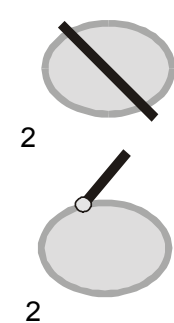

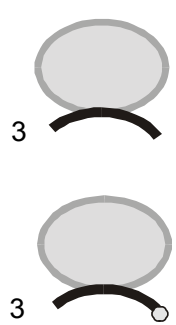

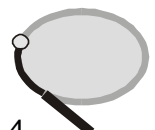

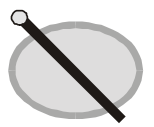

5

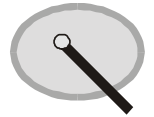

6

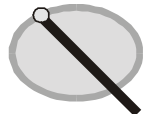

7

Abb. 72: Beziehungen eines Pfades zu polygonhaften Objekten: a) ohne, b) mit Berücksichtigung des Randes des Pfades.

Neben der Inhaltsinvariante der topologischen Beziehungen können auch andere Invarianten (Anzahl und Dimension der Komponenten) angewendet werden, welche die Beziehungen eines Graphen näher beschreiben. In einem Graph können bei Komponenten auch ihre Zugehörigkeit zur Kante untersucht werden: Die Fälle in Abbildung 73a beschreiben aus der Sicht der Inhaltinvariante und der Komponenten die gleichen Beziehungen, die jedoch in bezug auf die an der Beziehung beteiligten Kanten nicht gleich ist. Auch die Beziehungen des Knoten, der zu mehr als 2 Kanten inzident ist, können als Ausgangspunkt der Beschreibung topologischer Beziehungen eines Graphen dienen (Abbildung 73b). 
a)
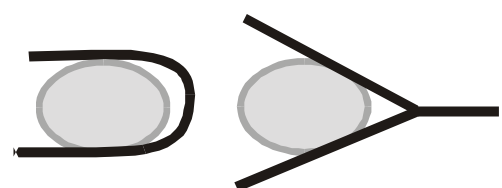

b)
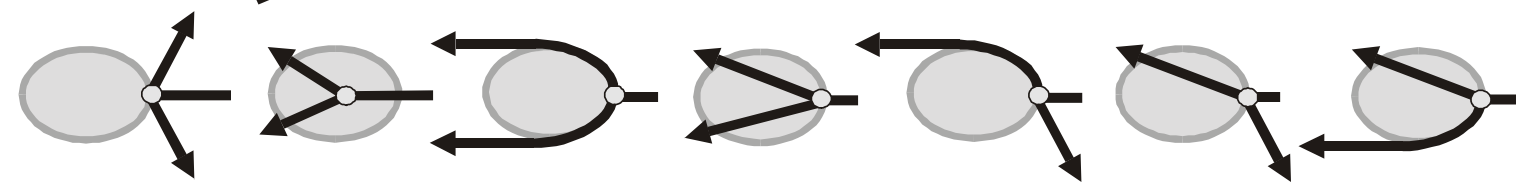

Abb. 73: Weitere topologische Beziehungen des Graphen: a) nach der Anzahl der beteiligten Kanten; b) nach der Position des Verzweigknoten.

Zur näheren Beschreibung der topologischen Beziehungen können auch Konzepte zur Quantifizierung räumlicher Beziehungen angewendet werden (s. Kap. 5.3.2.8)
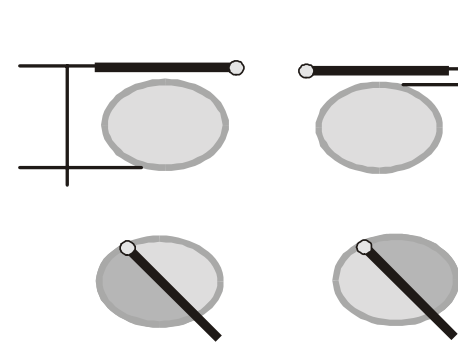

a)

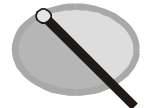

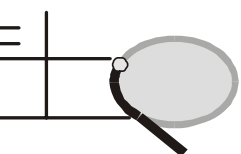

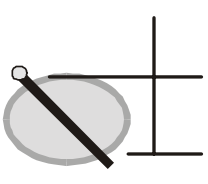

b)

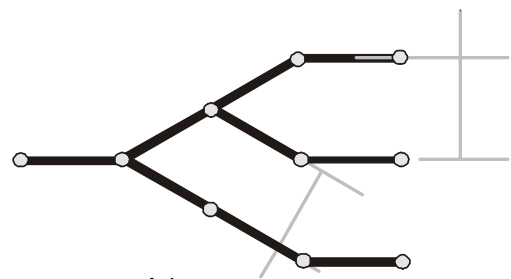

Abb. 74: Metrische Verfeinerung der räumlichen Konzepte: a) splitting und closeness; b) metrische Beschreibung der Kante-Kante Beziehung.

Die Beziehungen eines Wegenetzes zu dem Gelände stellen aus konzeptioneller Sicht Beziehungen eines Graphen zu einer Raumzerlegung (Tesselation) dar. Eine Tesselation ist eine den Raum vollständig füllende Komposition sich nicht überschneidender flächenhafter Objekte (Zellen) (Abbildung 75).

Eine Kante des Graphen kann eine oder mehrere Zellen schneiden und eine Zelle kann von einer oder mehreren Kanten geschnitten werden. Bei der ganzheitlichen Betrachtung des Wegenetzes (Graphen) als ein Objekt kann eine Zelle von dem Graphen geschnitten oder berührt werden, oder die Schnittmenge der Objektteile ist leer. Diese drei Fälle sind in der Abbildung 75 schematisch dargestellt.

\begin{tabular}{|c|l|l|}
\hline disjoint & intersect & \multicolumn{1}{c|}{ meet } \\
\hline$Z \cap G=\varnothing$ & $Z^{\circ} \cap G \neq \varnothing \wedge$ & $\partial Z \cap G \neq \varnothing \wedge$ \\
& $\partial Z \cap G \neq \varnothing$ & $Z^{\circ} \cap G=\varnothing$ \\
\hline
\end{tabular}



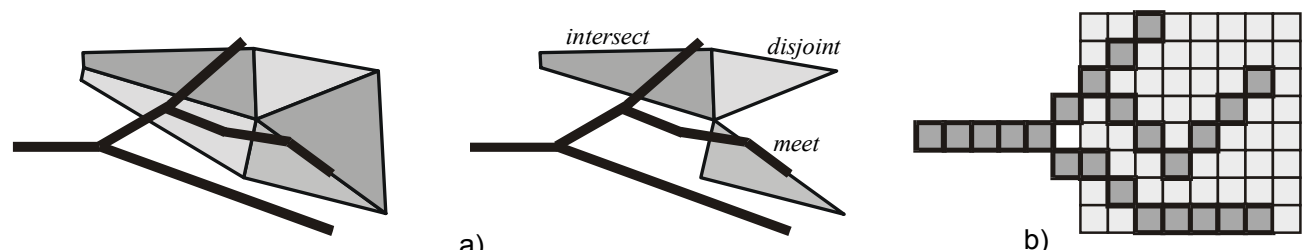

Abb. 75: Unterschiedliche Beziehungen eines Graphen und einer Tesselation: a) Beziehungen eines Graphen mit TIN Zellen; b) Repräsentation eines Graphen in Rasterdatenstruktur.

Die Anwendung und Interpretation der Richtungsbeziehungen in einem Graphen wird durch die Ausdehnung des Graphen erschwert. Wird der Graph als ein einfaches räumliches Objekt betrachtet, so können die Richtungsbeziehungen ,nach außen' und ,nach innen' unterschieden werden. Die Beziehungen ,nach außen' modellieren die Akzeptanzflächen der Beziehungen außerhalb der approximativen Ausdehnung des Objektes, die ,nach innen' diejenigen innerhalb der Approximation. Als approximative Ausdehnung des Graphen kann beispielsweise das minimal umgebende Recheck (minimal bounding rectangle MBR) angewendet werden. Bei den Beziehungen nach innen entscheidet die Art der Approximation über den Mittelpunkt des Graphen, von dem aus die Richtungen angegeben werden.

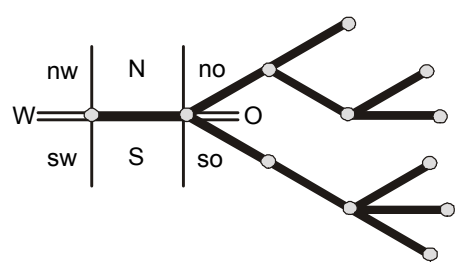

a)

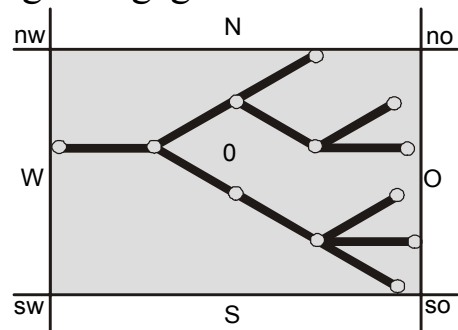

b)

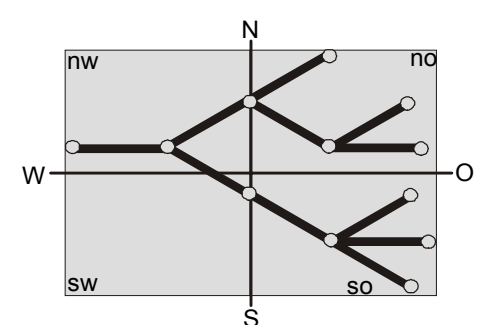

c)

Abb. 76: Richtungsmodelle des Graphen: a) Beziehungen der Kanten; b) projektionsbasierte Beziehungen ,nach außen'; c) projektionsbasierte Beziehungen ,nach innen'.

Die Richtungsbeziehungen nach außen spielen wegen der Größenordnungsbedingung keine wichtige Rolle. Alle oder fast alle räumliche Objekte befinden sich mindestens teilweise im neutralen Bereich der Approximation. Bei der Richtungsbestimmung nach innen wird die Approximationsfläche in Bereiche aufgeteilt, denen eine Richtung zugeordnet wird. Diesen Beziehungen kann eine punktförmige oder räumlich ausgedehnte neutrale Zone zugrunde liegen. Um dem willkürlich gewählten Mittelpunkt der Approximation an Gewicht zu nehmen, kann die neutrale Zone die gleiche Form wie die Approximation haben.

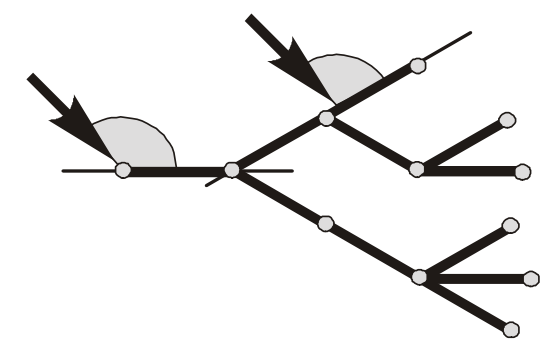

Abb. 77: Richtungsbeziehungen einer Kante bezogen auf eine vorgegebene Richtung (z.B. Windrichtung). 
Distanzbeziehungen können, je nach der konzeptionellen Fragestellung, gleiche oder ungleiche Bandbreiten zugrunde liegen (Abbildung 78). Ein wichtiges Konzept der Walderschließung ist die mehrfache Erschließung. Diese liegt vor, wenn eine Fläche in mehreren Bändern (Akzeptanzflächen) der gleichen Beziehung liegen. Das ist in Abbildung 78 schematisch dargestellt. Jede Kante des Graphen wird um ein räumliches Band einer Beziehung erweitert, wobei die Flächen mit mehrfacher Zugehörigkeit zu den Erschließungsbändern als mehrfach erschlossenen Fläche gilt.
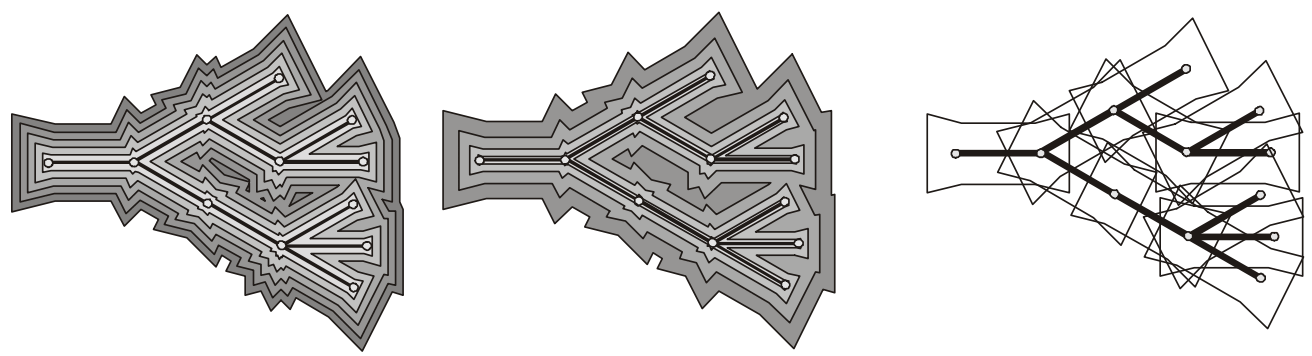

Abb. 78: Distanzbeziehungen eines Graphen: (links) equidistante Distanzbeziehungen, (mitte) ungleiche Akzeptanzflächen der Distanzbeziehungen, (rechts) Überlappung der Akzeptanzflächen.

\section{Kante/Kante Beziehungen}

Die Beziehungen der Kanten eines Graphen zueinander können ebenfalls als Kriterien zur Entscheidungsfindung verwendet werden. Aus der Sicht der Variantenbewertung stellen sie die eigenen Eigenschaften der Alternativen dar, sie beschreiben die Art der Zusammensetzung der Alternativen. In der Abbildung 79 sind einige Konzepte solcher Beschreibungen schematisch dargestellt.

Die Zusammensetzung kann auch mit räumlichen Beziehungsarten beschrieben werden. So beschreibt z.B. die kürzeste netzwerkbasierte Entfernung den Pfad zwischen zwei Knoten/Kanten. Die Anzahl der Kanten eines Pfades stellt den Grad der topologischen Adjazenzbeziehung dar. Zur Formbeschreibung des Weges kann die euklidische Entfernung in Verhältnis zur netzwerkbasierten Entfernung gesetzt werden.

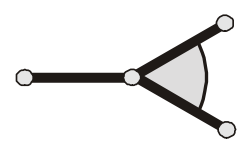

a)

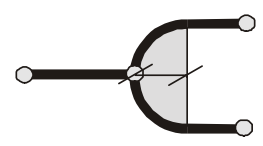

b)

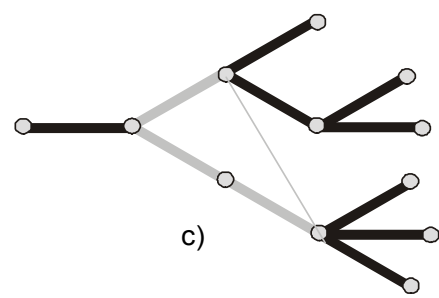

Abb. 79: Konzepte der Formbeschreibungen eines Pfades: a) Von zwei Kanten geschlossener Winkel; b) Radius eines von zwei Kanten umschlossenen Kreises; c) Vergleich der euklidischen und netzwerkbasierten Distanz zweier Knoten.

Neben den räumlichen Beziehungen können auch formbeschreibende Parameter eines Graphen angewendet werden. Der Winkel, der durch zwei adjazente Kanten gespannt wird oder der Durchmesser eines Kreises, welcher der Biegung einer oder mehrerer Kanten entspricht, können für die Alternativenbeurteilung von Bedeutung sein. 


\subsubsection{Entscheidungsfindung}

Die Entscheidung bezüglich der Erschließung eines Waldgebietes kann in zwei Formen auftreten. Die erste betrifft die Festlegung der Alternativen und entspricht somit der räumlichen Entscheidung FIND. Bei dieser Art der Entscheidung sind die Alternativen nicht von Anfang an bekannt und müssen zuerst gefunden werden. Die zweite Form der Entscheidung betrifft die Wahl zwischen zwei oder mehreren alternativen Erschließungen, die explizit vorgegeben sind und deren zielbezogene Vorteilhaftigkeit beurteilt werden muss. Diese Art der Entscheidung entspricht somit der räumlichen Entscheidung SELECT (vgl. Kap. 7.5.).

\section{Alternativensuche}

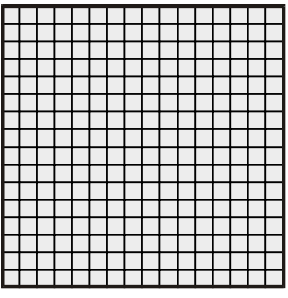

a) Zerlegung

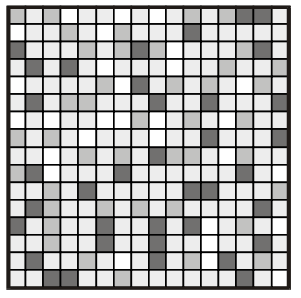

b) Raumbewertung

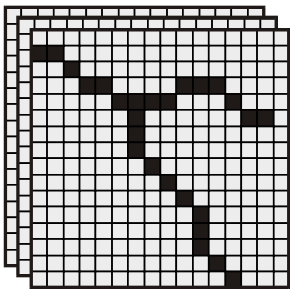

c) Komposition

Abb. 80: Schritte der Alternativengenerierung.

Die Generierung der Alternativen der Walderschließung erfolgt in drei Schritten: (i) Raumzerlegung, (ii) Raumbewertung und (iii) Komposition der Zellen. Während der Raumzerlegung wird der geographische Entscheidungsraum in Zellen zerlegt, welche die kleinste Raumauflösung darstellen. Eine Zelle ist somit eine Flächeneinheit mit homogenen entscheidungsrelevanten Eigenschaften. Bei der Raumbewertung wird jeder Zelle ein Präferenzwert zugeordnet, der den Beitrag dieser Flächeneinheit zur Zielerfüllung aus Sicht des Entscheidungsträgers beschreibt. Dieser stützt sich auf die räumlichen Beziehungen der Zelle. In dem letzten Schritt werden Alternativen gebildet, indem die möglichen und funktional-effizienten Kombinationen der Zellen gesucht werden.

\section{Variantenwahl}

Bei der Wahl einer Erschließungsvariante geht man von vorhandenen Alternativen aus und sucht diejenige, welche bezüglich ihrer Zielerfüllung den höchsten Präferenzwert besitzt. Zur Beurteilung einer alternativen Erschließung können die räumlichen Beziehungen des Wegenetzes mit forstlich relevanten räumlichen Objekten hinzugezogen werden. Für die Entscheidungssituation relevante Beziehungsarten können aus Tabelle 24 entnommen werden.

Tab. 24: Entscheidungsrelevante Beziehungsarten der Entscheidungssituation

\begin{tabular}{c|c|c|c|c|} 
& Waldeinheiten & DGM & Bodentyp & andere Objekte \\
\hline Wegenetz & $\begin{array}{c}\text { Topologie } \\
\text { Distanz } \\
\text { (Richtung) }\end{array}$ & Topologie & Topologie & $\begin{array}{c}\text { Topologie } \\
\text { Distanz }\end{array}$ \\
\hline
\end{tabular}

Die Güte der Walderschließung hängt in erster Linie von der Beziehungen der Waldeinheiten (Teilflächen) zum Wegenetz ab. Die Anzahl der direkt erschlossenen Waldeinheiten, die eine von „,disjoint“" verschiedene topologische Beziehung mit dem Wegenetz haben, kann als ein Konzept 
zur Beurteilung der Vorteilhaftigkeit von Alternativen angenommen werden. Als indirekt erschlossene Flächen können Waldeinheiten verstanden werden, die aufgrund ihrer topologischen Beziehungen als nicht direkt erschlossen und gleichzeitig aufgrund ihrer Distanzbeziehungen zum Wegenetz nicht als unerschlossen bezeichnet werden können. Als unerschlossene Waldeinheiten können solche Flächen bezeichnet werden, deren Distanzbeziehung zum Wegenetz einen Schwellenwert übersteigt, oder welche sich im Inneren von Objekten befinden, die als nicht überwindbar gelten. Indirekte Erschließung kann mit Distanzmodellen /min/ max/ durchschnittliche Entfernung\} modelliert werden, da es sich in der Regel um räumlich ausgedehnte Objekte handelt. Außerdem kann die Distanz nach der hangneigungsabhängigen Richtung (bergab, bergauf, höhenlinienparallel) spezifiziert werden. Die metrische Entfernungsgröße kann aber auch durch andere Ansätze (z.B. gewichtete Entfernung einer Kostenoberfläche) ersetzt werden.

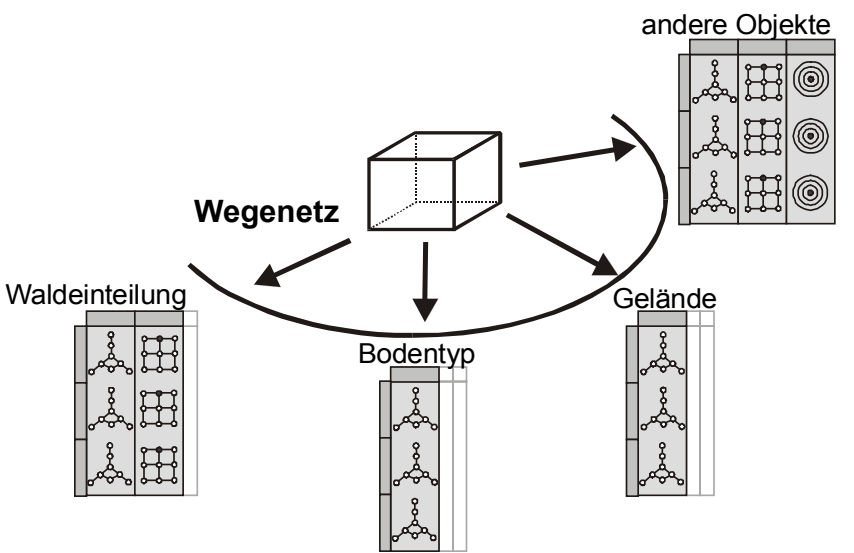

Abb. 81: Entscheidungssituation über Walderschließung.

Die Geländezugänglichkeit kann anhand der räumlichen Beziehungen eines Wegenetzes zu den Zellen einer Geländerepräsentation betrachtet werden. Dabei kommt es nicht nur auf die Beziehungen zu den Zellen selbst, sondern auch auf die Reihenfolge dieser Beziehungen an.

\subsubsection{Verjüngung}

\subsubsection{Motivation}

Die Waldverjüngung ist die Ablösung der alten Baumgeneration durch jüngere Bäume. Die Verjüngungsplanung als ein Teil der waldbaulichen Planung umfasst jene Maßnahmen, welche die planmäßige Erneuerung einer Waldgeneration auf bisher von Wald eingenommenen Flächen zum Ziel haben.

Die Walderneuerung wird durch den Verjüngungshieb eingeleitet und gesteuert. Die Stellung der Junggeneration gegenüber der alten sowie die zeit-räumliche Abfolge des Verjüngungsfortschrittes prägt das Tempo und das räumliche Muster der Walderneuerung (SCHÜTZ 1999). Die bevorstehenden Entscheidungen betreffen WO, WANN und WIE soll verjüngt werden. Das Ziel dieser Entscheidung ist sowohl Nutzen (Ernte) als auch dynamische Erhaltung des Ertragspotentials des Waldes. Räumliche Ordnung, deren Ziel es ist, eine "ordnunggemäße und nachhaltige Bewirtschaftung des Waldes durch planmäßige Ausnutzung und Beeinflussung 
der horizontalen und vertikalen Wald- und Bestandesgliederung maßgeblich zu fördern" (KURTH et al. 1994, S.76), kann nur durch Nutzungseingriffe hergestellt werden.

Tab. 25: Konzepte des Waldaufbaus (In Anlehnung an SCHÜTZE 1999, KURTH et al. 1994, MAYER 1992).

\begin{tabular}{l|l} 
Waldaufbauform & $\begin{array}{l}\text { Durch regelmäßige Anwendung charakteristischer Holzerntemaßnahmen und } \\
\text { Verjüngungsverfahren hergestellte typische Waldstrukturen: } \\
\text { Schlagfolgeordnung, Plenterordnung }\end{array}$ \\
Hieb & $\begin{array}{l}\text { Jeder Eingriff in die Bestockung, bei dem ein Baum oder mehrere Bäume bis } \\
\text { hin zu ganzen Beständen gefällt werden. } \\
\text { Dient dem Vorbereiten, Einleiten und Fortsetzen der Verjüngung sowie der } \\
\text { Ernte hiebsreifer Bäume. } \\
\text { Die Beziehung neuer zu alter Baumgeneration bei der Generationsablösung: } \\
\text { Kahlhieb, Schirmhieb, Saumhieb, Femelhieb, Plenterhieb. } \\
\text { Eine Fläche, auf der eine Hiebs- oder Verjüngungsmaßnahme in } \\
\text { Schlag }\end{array} \quad \begin{array}{l}\text { konzentrierter Form ausgeführt wird. Die flächenweise Führung der } \\
\text { Verjüngungshiebe führt zur Entstehung eines Schlages. } \\
\text { Unterteilung des Waldes in Schläge, auf denen der Baumbestand besonders } \\
\text { altersmäßig ziemlich einheitlich ist und auf denen waldbauliche Maßnahmen } \\
\text { (Verjüngung, Pflege, Ästung, Durchforstungen usw.) zeitlich und räumlich } \\
\text { isoliert voneinander stattfinden. } \\
\text { Charakterisiert dadurch, daß alle Entwicklungstufen auf kleinster Fläche } \\
\text { nebeneinander stehen. }\end{array}$
\end{tabular}

Die Entscheidungen über Walderneuerung stellen ein komplexes Problem dar, das nicht eindeutig lösbar ist, "da ökologische, betriebs- und volkswirtschaftliche, überwirtschaftliche und waldbautechnische Aspekte, exakte und nur gutachtlich faßbare Wertungen gleichzeitig zu optimieren sind" (MAYER 1992, S.298).

Die Entscheidungen über Verjüngung werden wegen der Komplexität der betroffenen Entscheidungsprobleme sowie ihren vielfältigen Interdependenzen daher meistens subjektivempirisch getroffen. Die Nutzungsentscheidungen stützen sich auf Erfahrung, visuellen Eindruck und Einfühlungsvermögen, die bisher nur unzureichend formalisierbar sind.

Tab. 26: Konzepte der räumlichen Ordnung des Verjüngungsablaufes (In Anlehnung an SPEIDEL 1972, KURTH et al. 1994, MAYER 1992).

\begin{tabular}{l|l} 
seitliches Schützen & $\begin{array}{l}\text { Nachbarlicher Schutz bei seitlich schräg auftreffenden Faktoren: Sturm, } \\
\text { Windeinwirkung, Untersonnung, Verhagerung, Rauhfrost, } \\
\text { Immissionsschäden }\end{array}$ \\
Nachbarschaftsbeziehungen, \\
Deckungsschutz \\
Lagerung von Beständen zum Zweck des seitlichen Schutzes. Die \\
Nachbarschaftsbeziehungen treten dort auf, wo zwei Bestände etwa \\
linearaneinander grenzen. Der Umfang des Deckungsschutzes kann in \\
Bestandeskomplex \\
Schlagreihe \\
Gruppe von Beständen mit Nachbarschaftsbeziehungen \\
Hezifische Form des Bestandeskomplexes mit Nachbarschafts- \\
beziehungen zwischen den Bauteilen. Eine Reihe von Beständen, die \\
sich in der Richtung der Hauptgefahr aneinander reihen und dieser \\
entgegengesetzt genutzt werden. \\
Eine dauernd fest begrenzte Waldfläche, für die nach außen \\
Selbständigkeit der Bestockung durch Trauf, im Inneren eine solche \\
Altersabstufung in bestimmter Richtung angestrebt und erhalten wird, \\
dass sich die Schläge ungefährdet in gesamtwirtschaftlich \\
zweckmäßiger Weise folgen können.
\end{tabular}


Im folgenden wird die räumliche Situation der Waldverjüngung schematisch beschrieben. Es wird auf die Beziehungen und räumlichen Objekte eingegangen, die für eine Entscheidungsfindung in dieser komplexen Handlungssituation von Bedeutung sein können. Ein integrales Entscheiden ist nicht möglich, eine Dekomposition der Teilaspekte der Waldverjüngung ist zwar möglich, ihre Zusammenführung jedoch wegen vielfätiger Interdependenzen sehr schwierig. Die folgende Beschreibung kann als Skizze einer räumlichen Entscheidungsunterstützung dienen, die von einem GIS geleistet werden kann.

\subsubsection{Modellierung der räumlichen Situation}

Die Verjüngung wird auf einer im Vergleich mit einem Bestand größeren Waldfläche konzipiert, um eine kohärente räumliche Ordnung zu gewährleisten. Diese Verjüngungsfläche wird nicht auf einmal verjüngt, sondern in räumlich und zeitlich gestaffelten Operationen. Die Teile der Verjüngungsflächen, auf welchen die Verjüngungsschritte gleichzeitig erfolgen, werden Teilflächen genannt (Abbildung 82). Die Hiebsarten sind das technische Instrumentarium zur Optimierung des Erneuerungsprozesses, ein Hieb stellt ein Ereignis der räumlichen Situation dar.
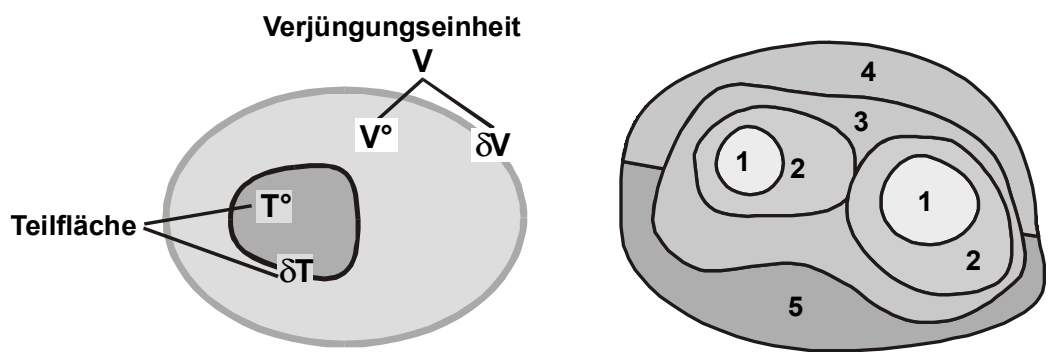

Abb. 82: Konzepte der Walderneuerung: (links) Teilfläche und Verjüngungsfläche, (rechts) komplexe räumliche Situation und zeitlicher Ablauf der Verjüngung am Beispiel des Femelschlages.

Die räumliche Situation der Verjüngungsplanung ist äußerst komplex. In der Regel wird Naturverjüngung mit mehreren in Art, Form und Stärke unterschiedlichen Auflösungen des Altbestandes durchgeführt (SCHÜTZ 1999). Die Verjüngungsstellung wird den konkreten Gegebenheiten (Baumarten und ihre Zusammensetzung, Standort, Gelände, etc.) angepasst.

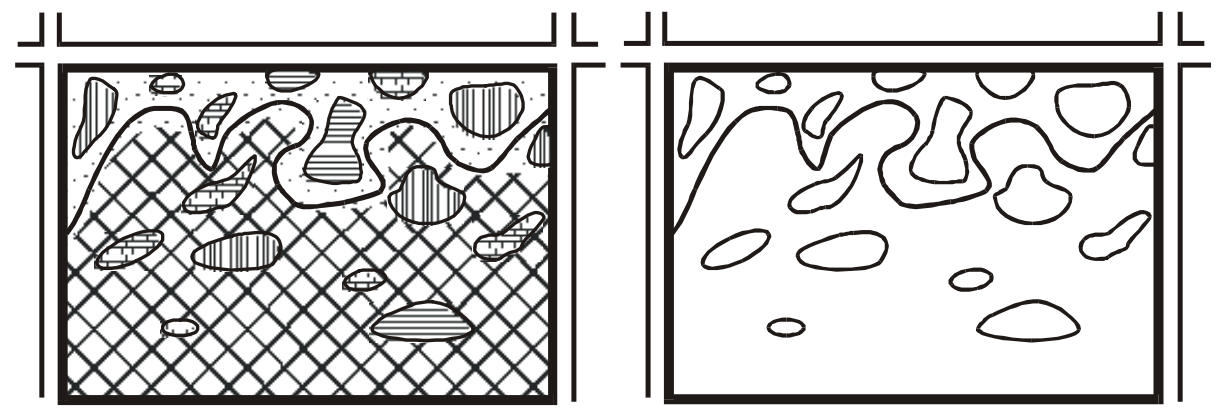

Abb. 83: Komplexität der Walderneuerung am Beispiel des Saumfemelschlags (eigene Graphik nach SEEHOLZER 1922 In: MAYER 1992): (links) ursprüngliche Situation nach SEEHOLZER, (rechts) eine Vereinfachung der räumlichen Situation.

Die jeweils geräumten Flächen und die zeitliche Folge dieser bilden den Grundriß und Aufriß der Bestockung (KURTH et al. 1994, S.108). Während der Verjüngung entstehen räumliche Objekte unterschiedlicher Form, Größe und Ausrichtung, deren Beziehungen zueinander den Grundriß und Aufriß der Bestockung prägen. Die Komplexität einer Verjüngungssituation ist auch in 
Abbildung 83 deutlich zu erkennen, welche das Prinzip des Saumfemelschlages (MAYER 1992) schematisch beschreibt.

Die wichtigsten Beziehungen der räumlichen Situation bei Walderneuerung sind die räumlichen Beziehungen einer Verjüngungsfläche und ihrer Teilflächen (s. Abbildung 84:1), sowie der Teilflächen zueinander (Abbildung 84:2). Dabei können alle Beziehungsarten (Topologie / Distanz / Richtung) oder ihre Kombination von Bedeutung sein. Als für die Handlungssituation relevante räumliche Objekte kommen u.a. Boden- und Standorteinheiten, Wegenetz und Transportgrenze sowie Geländeform in Betracht. Die räumlichen Beziehungen der Teilflächen zu Standortseinheiten bzw. zum Wegenetz oder zur Transportgrenze sind in Abbildung 84 (:3 bzw. :4) schematisch dargestellt.

Tab. 27: Zusammenfassung entscheidungsrelevanter Beziehungen von Verjüngungsflächen.

\begin{tabular}{l|c|l} 
& \multicolumn{2}{|c}{ Verjüngungsfläche $\left(\mathbf{V}_{\mathbf{i}}\right)$} \\
\hline \multicolumn{1}{c|}{ Objekt/ } & Geometrietyp & \multicolumn{1}{c}{ Beziehungen } \\
\hline Teilfläche $\left(\mathrm{T}_{\mathrm{j}}\right)$ & $\mathrm{P} / \mathrm{P}$ & covers, in, equal \\
Standortseinheit $\left(\mathrm{S}_{\mathrm{j}}\right)$ & $\mathrm{P} / \mathrm{P}$ & $\begin{array}{l}\text { equal, overlap, covers, coveredBy, } \\
\text { in, contain, }\end{array}$ \\
Waldeinheit $\left(\mathrm{W}_{\mathrm{j}}\right)$ & $\mathrm{P} / \mathrm{P}$ & equal, covers, contain, \\
Wegenetz, Transportgrenze & $\mathrm{L} / \mathrm{P}$ & 3 (siehe Abbildung 72a) \\
Gelände & $\mathrm{P} / \mathrm{P}$ & 3 (siehe Abbildung 75$)$
\end{tabular}
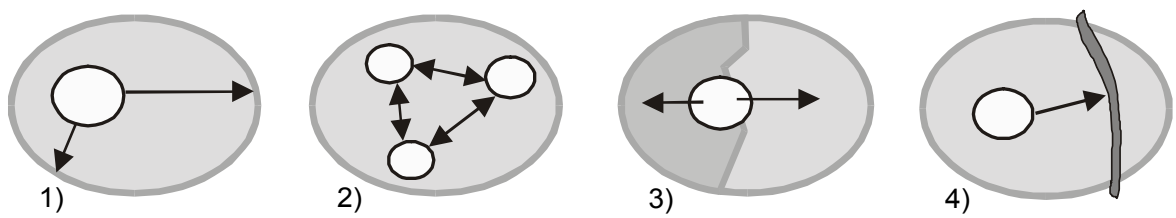

Abb. 84: Relevante Beziehungen der räumlichen Situation bei Walderneuerung: 1) Beziehungen einer Teilfläche zur Verjüngungsfläche; 2) Beziehungen der Teilflächen zueinander; 3) Beziehungen einer Teilfläche zu Standortseinheiten; 4) Beziehungen einer Teilfläche zu Wegenetz/Transportgrenze.

Die möglichen topologischen Beziehungen zwischen einer Verjüngungsfläche und einer Teilfläche werden durch die Bedingung des Enthaltenseins der Teilflächen $\left(T_{i}\right)$ in der Verjüngungsfläche $(\mathrm{V})$ eingeschränkt. Die Bedingung lässt nur drei topologische Beziehungen der Inhaltsinvariante für Polygon/Polygon-Objekte zu (siehe Abbildung 85): \{covers, in, equal\}. Die Beziehung \{covers\} lässt sich weiter nach der Dimension der Rand-Rand Komponente ( $\partial \mathrm{V} \cap$ $\partial \mathrm{T})$ sowie der Anzahl von $\left(\mathrm{V}^{\circ} \cap \mathrm{T}^{\top}\right)$ Komponenten verfeinern.
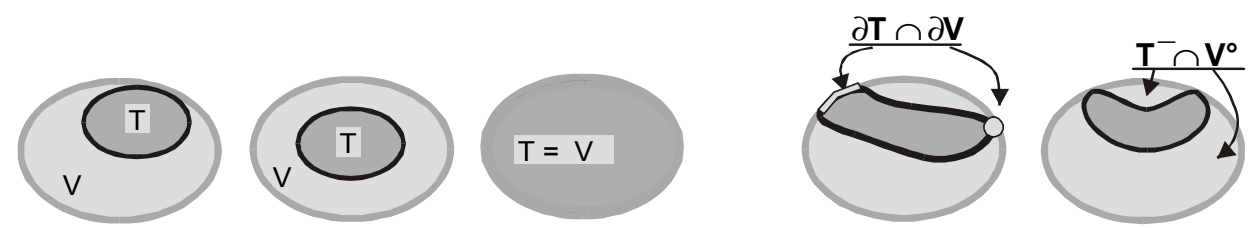

Abb. 85: Topologische Beziehungen der Inhaltsinvariante zwischen Verjüngungsfläche (V) und der Teilfläche (T).

Die topologischen Beziehungen der Teilflächen zueinander müssen die Bedingung der leeren $\left(T_{i}^{\circ} \cap T_{j}^{\circ}\right)$ Schnittmenge erfüllen. Die möglichen Beziehungen der Inhaltsinvariante sind dann 
$\{$ disjoint, meet $\}$. Die Beziehung $\{$ meet $\}$ kann dabei eine Spezialform annehmen, wenn der innere Rand der Teilfläche $i$ sich mit dem äußeren Rand der Teilfläche $j$ schneidet (Abbildung 86). Darüber hinaus kann sie auch mit weiteren Invarianten verfeinert werden.

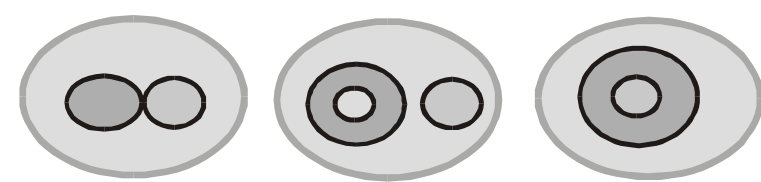

Abb. 86: Topologische Beziehungen der Teilflächen zueinander nach der Inhaltsinvariante.

Die Teilflächen können zu Standortseinheiten in folgenden Beziehungen stehen: \{disjoint, meet, overlap, coveredby, in\}. Es wird vorausgesetzt, dass die Teilflächen kleiner sind als Standorteinheiten. Daher werden die Beziehungen \{equal, covers, contains\} ausgeschlossen.

Tab. 28: Zusammenfassung der entscheidungsrelevanten Beziehungen von Teilflächen.

\begin{tabular}{l|c|l}
\multicolumn{1}{c|}{ Objekt/ } & \multicolumn{2}{|c}{ Teilfläche $\mathbf{~}_{\mathbf{i}} \mathbf{)}$} \\
\hline Teilfläche $\left(\mathrm{T}_{\mathrm{j}}\right)$ & Geometrietyp & \multicolumn{1}{c}{ Beziehungen } \\
Standorteinheit $(\mathrm{S})$ & $\mathrm{P} / \mathrm{P}$ & meet, disjoint \\
Wegenetz, Transportgrenze & $\mathrm{P} / \mathrm{P}$ & intersect, meet, covers, in, equal \\
Gelände & $\mathrm{L} / \mathrm{P}$ & 3 (siehe Abbildung 72a) \\
& $\mathrm{P} / \mathrm{P}$ & 3 (siehe Abbildung 75)
\end{tabular}

Die Beziehungen der Teilflächen sowie der Verjüngungseinheit zu Wegenetz bzw. Transportgrenze entsprechen den Beziehungen in Abbildung 72 (Kap. 8.3.3). Es sind nur drei unterschiedliche Beziehungen der topologischen Inhaltsinvariante möglich, da der Rand der Transportgrenze bzw. des Weges für diese Fragestellung nicht von Bedeutung ist.

Für die Bestimmung von Distanzbeziehungen zwischen räumlich ausgedehnten Objekten muss ein Distanzmodell (min, max, mini-max, average; s. Kap. 5.3.3) gewählt werden. Die Richtungsbeziehungen der Teilflächen sowie ihrer Anordnung werden vor allem in bezug auf eine vorgegebene Richtung (Wind- und Sturmrichtung) bestimmt. Die Geländeform (Exposition und Neigung) beeinflusst die Lichtverhältnisse in Teilflächen und bestimmt daher die Präferenzen bei der Anordnung (Richtungsbeziehungen) der Teilflächen. In Abbildung 87 sind Beziehungsmodelle zwischen den Teilflächen und der Verjüngungseinheit dargestellt.

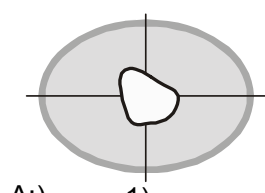

A:)

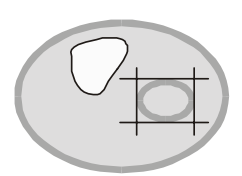

2)

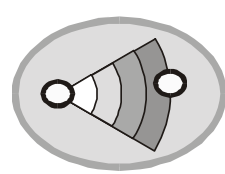

3)

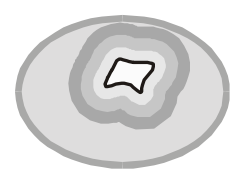

4)

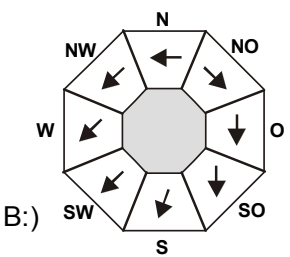

Abb. 87: Schematische Darstellung der räumlichen Beziehungen zwischen den Teilflächen und der Verjüngungseinheit: A) Richtungsbeziehungen (1) der Verjüngungsfläche 'nach innen', (2) der Teilflächen; (3) Distanzbeziehungen zwischen den Teilflächen, (4) zwischen der Teilfläche und der Verjüngungsfläche; B) Hiebsrichtung in Abhängigkeit von Geländeneigung, Hiebsschüssel (nach WAGNER 1911, zitiert nach KURTH et al. 1994, vereinfacht). 
Form, Größe und Ausrichtung der Verjüngungshiebe sind ebenfalls von Bedeutung. In ihrer Zusammenwirkung beeinflussen sie Prozesse (Lichtregime, Bodenfeuchtigkeit, Extremtemperaturen, usw.), welche die Verjüngung steuern oder zumindest beeinflussen können.

Die Beziehungen der Teilflächen zueinander beschreiben den Fortschritt der Verjüngung: Neben den Beziehungen bezüglich der $x y$ Koordinaten (Grundriß der Bestockung) sind auch Beziehungen bezüglich der $z$ Koordinate (Aufriß der Bestockung) von Bedeutung.

N

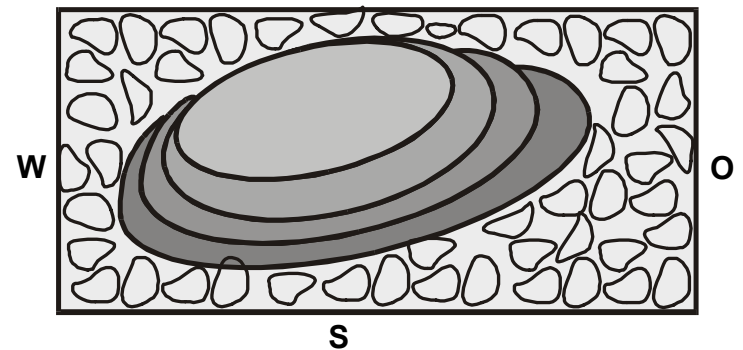

$\mathbf{N}$

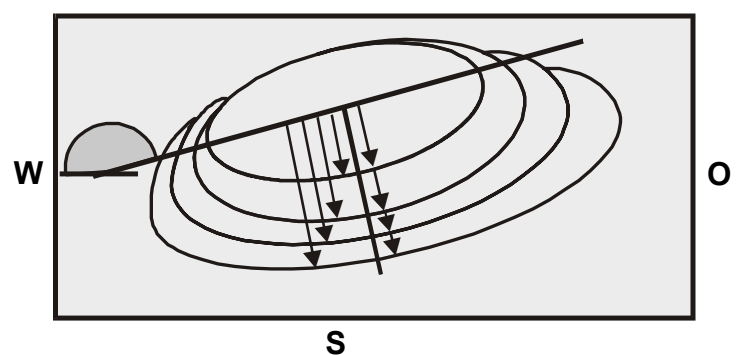

Abb. 88: Beziehungen der Teilflächen zueinander (in Anlehnung an VANSELOW 1949, zitiert nach MAYER 1992).

Diese werden anhand der Bestandeshöhe oder des Alters für Bestände dargestellt. Sie müssen eine Bedingung erfüllen, welche die Anzahl möglicher Beziehungen auf 3 einschränkt: Die bezüglich der dritten Dimension zu vergleichenden räumlichen Objekte haben alle den gleichen Anfang - die Erdoberfläche bzw. den 0-Referenzpunkt für das Alter. Die möglichen Beziehungen $\{$ equal, overtops, overtopped_by $\}$ können mit den topologischen Beziehungen in der Ebene kombiniert werden, wie das in Abbildung 89 dargestellt ist. Diese Beziehungen können die verschiedenen Grundstellungen bei der Generationsablösung bzw. bei den Hiebsarten (Kahlhieb, Schirmhieb, Saumhieb, Femelhieb) modellieren.

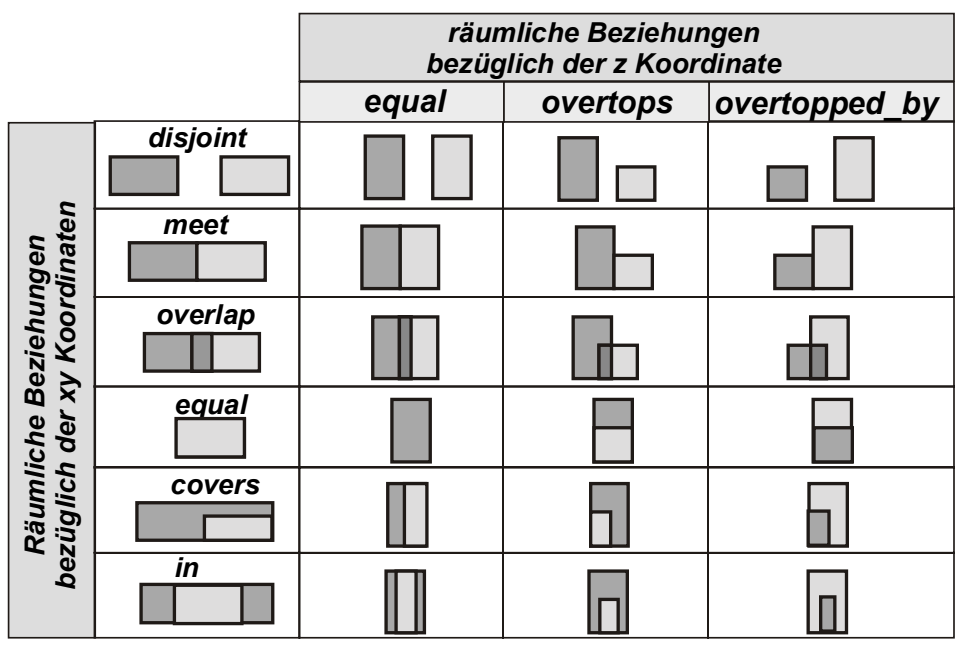

Abb. 89: Räumliche Beziehungen bezüglich der Höhe (z Koordinate).

Die Anordnung der Teilflächen zueinander, festgelegt durch Art, Intensität und Tempo der Verjüngung, bestimmt die räumliche Ordnung des Waldaufbaus. Bei dem Aufbau der räumlichen Ordnung im schlagweisen Wald (,räumliche Ordnung im Großen“, KURTH et al. 1994) sind 
Beziehungen der Schläge in einer Schlagreihe zueinander, der Schlagreihen eines Hiebszuges zueinander sowie der Hiebszüge zueinander von Bedeutung. Beispiele dieser Beziehungen sind in Abbildung 90 in Deckungsschutzsystemen des Fachwerkes schematisch dargestellt (WAGNER 1924, zitiert nach KURTH et al. 1994).
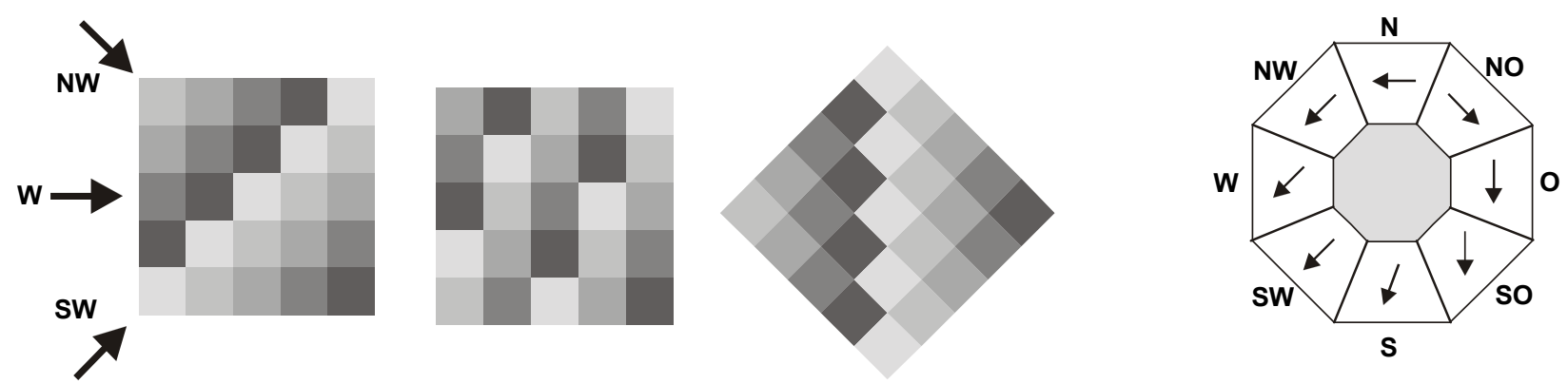

Abb. 90: Deckungsschutzsysteme für das Fachwerk (nach WAGNER 1928 zitiert nach KURTH et al. 1994, verändert).

\subsubsection{Entscheidungsfindung}

Wegen der Komplexität der Zusammenhänge ist bei der Waldverjüngung nicht die Entscheidungsfindung im engen Sinne (Abbildung der Präferenzen), sondern eher die Entscheidungsunterstützung im weiteren Sinne sinnvoll: Bereitstellung und Handhabung räumlicher Informationen, Modellierungsunterstützung zur Erstellung von Szenarien und Alternativen etc. Nicht der schablonenhaften Durchsetzung generalisierter Schemata, sondern einer kreativen Suche (Exploration) nach Lösungsmöglichkeiten soll hier der Vorzug gegeben werden.

Die Verjüngungsplanung bedeutet Hiebsart, Position, Form und Anzahl der Teilflächen und ihre zeitliche Folge festzulegen. Die bevorstehenden Entscheidungen in der Verjüngungsplanung betreffen die Position \{WO\} der Verjüngung, den Zeitpunkt ihres Anfanges \{WANN\} sowie Art und Fortschritt $\{\mathrm{WIE}\}$.

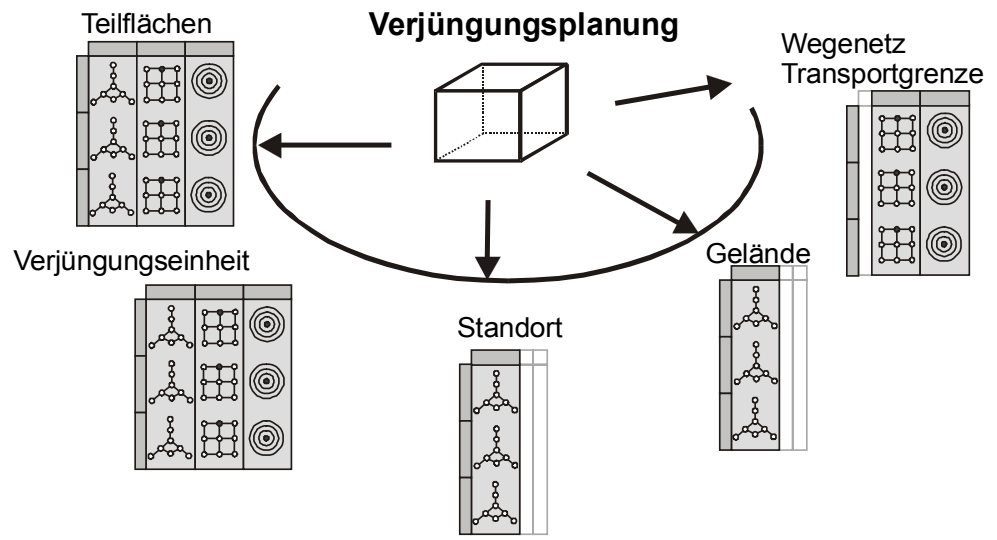

Abb. 91: Entscheidungssituation der Verjüngungsplanung.

Aus der räumlichen Sicht sind vor allem folgende Entscheidungen von Interesse: 
- Die Abgrenzung der Verjüngungsfläche ist eine Nutzungsentscheidung im Raum-ZeitGefüge. Die Hiebsreife der Bestände und die beabsichtigte räumliche Ordnung sind zu berücksichtigen.

- Entscheidungen über die Einleitung der Verjüngung betreffen die Anzahl, Form und Größe der Teilflächen. Die Naturverjüngungswirtschaft stellt besondere Anforderungen an Form und Größe der Schläge.

- Entscheidungen über die Fortsetzung der Verjüngung sind Entscheidungen über die räumliche Anordnung der Verjüngung. Eine bestimmte Folge und räumliche Disposition der Teilflächen wird als räumliche Anordnung (räumliche Ordnung) der Verjüngung bezeichnet (SCHÜTZ 1999).

Entsprechend den oben genannten Entscheidungen kann die Fragestellung der konkreten Entscheidungssituation unterschiedlich geäußert werden. Die Handlungsfreiheit hängt dann davon $\mathrm{ab}$, ob es sich um eine Einleitung oder Fortsetzung der Verjüngung handelt. Die einzelnen Entscheidungen weisen eine Abhängigkeit auf. Bei der Festlegung der Verjüngungseinheit oder später bei der Entscheidung über die Einleitung der Verjüngung kann der Verjüngungsablauf oder -fortschritt impliziert werden.

Die Entscheidung über die Abgrenzung der Verjüngungseinheit ist eine räumliche Entscheidung in dem Sinne, dass dabei die Nutzungsreife der einzelnen Bestände mit ihrer räumlichen Lage in dem räumlichen Gefüge abgewogen werden muß. Ein Bestand verliert um so mehr an Selbständigkeit, je mehr er z.B. auf seitlichen Schutz (Nachbarschaftsbeziehungen) angewiesen ist.

Die Entscheidung über die Einleitung und Fortsetzung der Verjüngung ist eine räumliche Entscheidung, da Position, Form, Größe und Ausrichtung der Teilflächen so gesucht werden, dass eine kohärente räumliche Ordnung gewährleistet wird.
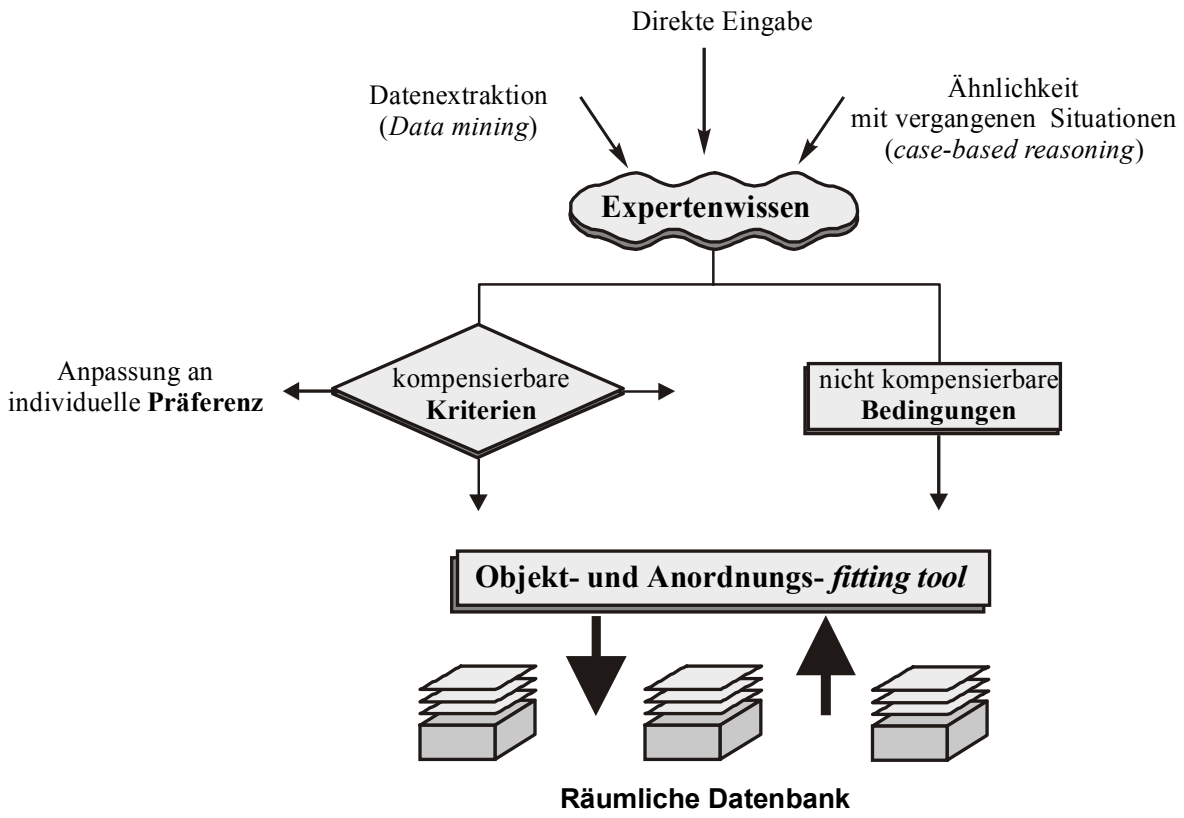

Abb. 92: Schematische Darstellung der räumlichen Entscheidungsunterstützung bei Verjüngungsplanung. 
Die Entscheidungsunterstützung bei der Verjüngungsplanung kann als Bereitstellung von Expertenwissen und Modellierungswerkzeug dem Entscheidungsträger dienen (s. Abbildung 92). Das Expertenwissen kann direkt angegeben oder aus ähnlichen Handlungssituationen in der Vergangenheit aus einer räumlichen Datenbank extrahiert werden. Dieses wird nach seiner Bedeutung für die gegebene Handlungssituation in nicht-kompensierbare (harte) Kriterien (constraints) und kompensierbare (weiche) Kriterien eingeteilt. Die weichen Entscheidungskriterien bieten einen Spielraum für die Äußerung individueller Präferenzen des Entscheidungsträgers.

Die Kriterien und Bedingungen werden weiter an ein Modellierungswerkzeug (fitting tool) gegeben, mit welchem man den Entscheidungsraum nach einer, optimalen' Position und Anordnung der Teilflächen interaktiv durchsuchen kann. In der Abbildung 93 wird die interaktive Suche unterschiedlicher Eigenschaften eines Objektes oder einer Anordnung dargestellt.
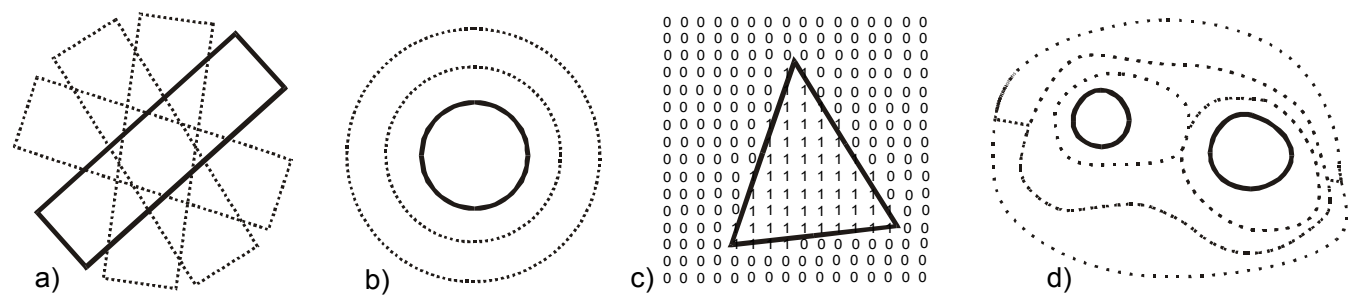

Abb. 93: Objekt- und Anordnung - Suche: a) Ausrichtung des Vierecks; b) Größe des Kreises; c) Position des Objektes mit Hilfe einer Shape-Matrix (vgl. FLUSSER 1991); d) Anpassung der Anordnung von Objekten.

\subsection{Schlussfolgerung zur Anwendung der Geoinformationen in der Forstwirtschaft}

Forstliche Planung ist eine räumliche Planung: Erscheinungsformen und Strukturierungen des Waldes lassen sich nur durch Einordnung in Raum-Zeit-Beziehungen erklären (KURTH et al. 1994). Die Waldbewirtschaftung ist eine flächenweise differenzierte Steuerung der natürlichen Prozesse, deren Ziel eine ausgewogene Erfüllung der Nutz-, Schutz- und Erholungsfunktion des Waldes ist.

Das prägende Prinzip der Forstwirtschaft ist das Streben nach Nutzen sowie nach seiner Sicherheit. Die inhärente Räumlichkeit der Forstwirtschaft ist durch die räumliche Prägung des Nutzens charakterisiert: Durch die räumlichen Objekte und ihre Beziehungen, an welcher der Nutzen gemessen wird. Die Räumlichkeit des Nutzens wird durch räumliche Beziehungen beschrieben und lässt sich anhand dieser in andere Größen überführen. Monetäre oder zeitliche Größen stehen hierfür beispielsweise zur Verfügung.

Räumliche Präferenz in der Forstwirtschaft ist Ausdruck räumlicher Auflösung eines Zieles. Sie stützt sich notwendigerweise auf die räumlichen Konzepte, welche im Rahmen des jeweiligen Paradigmas der Waldregelung von Bedeutung sind. In den forstlichen Entscheidungen ist die räumliche Präferenz nicht transparent. Dem 'Gesetz des Örtlichen', das die Standortsbezogenheit aller Maßnahmen prägt, steht das Streben nach 'räumlicher Ordnung' gegenüber, welches die örtlichen Schadbedrohungen mindert sowie Einteilung und Erschließung des Waldes vornimmt. 
Die Sicherheit des Nutzens enthält räumliche Aspekte, ihre Präferenzen werden jedoch in nicht kompensierbarer Form als strikte Anweisungen und Bedingungen formuliert.

Der wachsende Druck zur Bildung räumlicher Präferenz in der Forstwirtschaft scheint von außerhalb zu kommen. Die zunehmenden Konflikte der Raumnutzung, die Berücksichtigung von außerbetrieblichen Interessen bei der Waldregelung führen dazu, dass forstliche und forst-fremde räumliche Konzepte in der Entscheidungsfindung kombiniert werden müssen. Jede Entscheidungsfindung setzt aber die Existenz einer zielbezogenen Präferenz des Entscheidungsträgers voraus. Die Einbeziehung fremder Konzepte in die Entscheidungsfindung, gegenüber der die Entscheidungsträger (Förster) keine oder nur unvollständige Präferenz besitzen, führt zu ,quasi“ Entscheidungen, die weder transparent noch rational sind.

Die behandelten Beispiele stellen nur eine kleine Auswahl räumlicher Handlungssituationen in der Forstwirtschaft dar: Waldeinteilung, Funktionszuordnung, Durchforstung u.a. besitzen ebenfalls räumliche Aspekte, welche Gegenstand einer Entscheidung werden können.

Für das bessere Verständnis der Räumlichkeit, sowie für die Vollständigkeit und die Transparenz einer Entscheidungssituation und ihrer computergestützten Abbildung ist es nötig, räumliche Konzepte $\mathrm{zu}$ formulieren und für die Implementierung zu formalisieren. Die formalen bzw. formalisierten räumlichen Konzepte können jedoch einem Entscheidungsträger zu eng sein, wenn sie nicht genau seine Absicht und Zielvorstellung zum Ausdruck bringen: Die Diskrepanz subjektiver und nicht transparenter Konzepte auf einer Seite und objektiv formaler und widerspruchsfreier Konzepte auf der anderen, trägt zusätzlich zur Komplexität räumlicher Entscheidungsprobleme bei. Die räumliche Entscheidungsfindung muss die Konzepte immer auf ihre Vollständigkeit und Aussagekraft für den Entscheidungsträger prüfen.

Versteht der Entscheidungsträger die räumliche Situation nicht, oder vermisst er in ihr einen für die Entscheidung relevanten Aspekt, so kann seine Bereitschaft zur Äußerung der Präferenzen oder zur Annahme einer vorgeschlagenen Lösung sinken. In anderen Fällen kann auch das Gegenteil auftreten, wenn er aus mangelnder Übersicht und Präferenzbewusstsein eine durch computergestützte Datenverarbeitung vorgeschlagene Lösung unkritisch und oft falscherweise als seinen Präferenzen am besten entsprechend annimmt.

Trotz der vielfältigen Anwendungsmöglichkeiten wird das Einsatzpotential von Geographischen Informationssystemen nicht ausreichend genutzt. Das hat vermutlich Gründe in folgenden Bereichen:

- mangelnde Akzeptanz der Informationstechnologie wegen Systemfehlern

- mangelnde Akzeptanz der Informationstechnologie wegen mangelnder Konzeptbildung

Die Computertechnologie hat immer noch mit fehlender Akzeptanz als Reaktion auf die fehlgeschlagenen Euphorien bezüglich ihres Einsatzes in der Vergangenheit zu kämpfen. Dies gilt in gleicher Weise auch für GIS. Wegen ihrer oft schwierigen Bedienbarkeit und der hohen Komplexität waren sie bis vor kurzem nur einem engen Kreis der „Experten“ vorbehalten. Durch neuere Entwicklungen, die vor allem auf die Forschungsergebnisse der GIScience zurückzuführen sind, werden die Geographischen Systeme immer benutzerfreundlicher und somit einem breiteren Benutzerkreis zugänglich. Die Internetfähigkeit der GIS erlaubt schließlich jedem, seinen Kenntnissen entsprechend auf die Geoinformationen zuzugreifen und sie zu verarbeiten. Der Informationsaustausch in den proprietären Systemen war bis vor kurzem 
unmöglich oder mangelhaft. Durch Standardisierung können offene GIS Produkte (OpenGIS) entwickelt werden, welche Daten aus unterschiedlichen Quellen beliebig verknüpfen können.

Das Verstehen der Geoinformationen ist untrennbar mit dem Verstehen ihrer Konzepte verbunden. Das modellbasierte Denken, in dem die Konzepte aus der Realität abstrahiert, in Datenmodellen abgebildet und schließlich verarbeitet werden, muss erworben bzw. erlernt werden. Fehlende Kompetenz im Verstehen und Handeln mit räumlichen Konzepten kann zu Ablehnung oder zu übertriebenen Erwartungen auf Seiten des Benutzers führen. Die Übernahme von fremden Konzepten ohne ausreichende oder sogar fehlerhafte Interpretation ist genauso gefährlich wie falsche oder gefälschte Informationen. Die Präferenz bezüglich fremder Konzepte kann nur langsam gebildet werden, wenn man sie vollständig versteht und ihre Bedeutung anerkannt wird. 


\section{Anwendung räumlicher Entscheidungsfindung am Beispiel des Harverstereinsatzes}

\subsection{Einführung in die Entscheidungssituation}

Das folgende Beispiel zeigt eine Anwendung räumlicher Entscheidungsfindung an einem einfachen forstlich relevanten Entscheidungsproblem. Das Beispiel wurde bereits in den Kapiteln 2.4 und 8.3 angesprochen. Es wurde gewählt, um den Entscheidungsprozess in räumlicher Domäne zu konkretisieren. Die Fragestellung dieses Beispieles stammt aus SpORS et al. (1992).

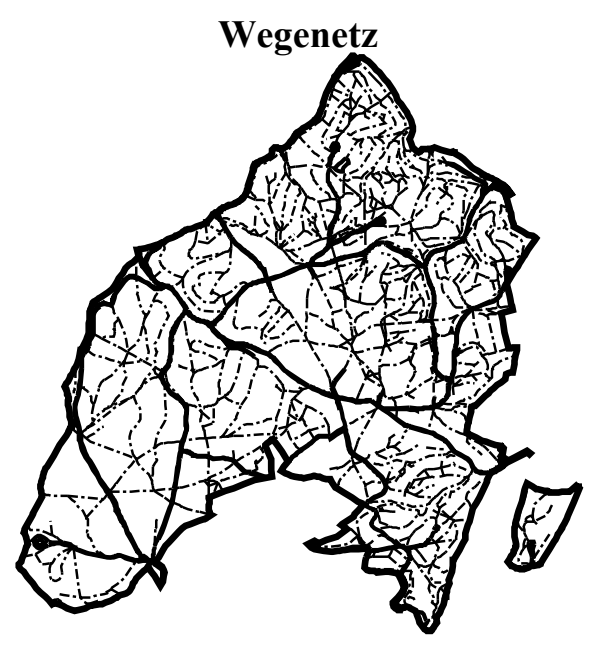

$1: 60000$

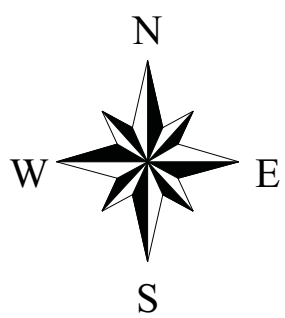

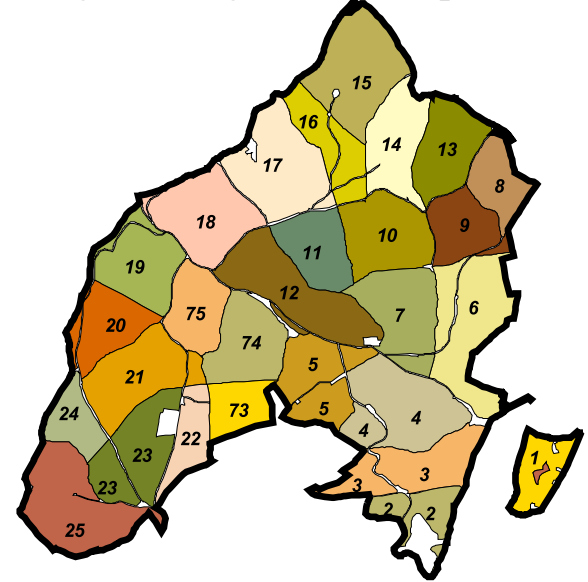

Forsteinrichtung

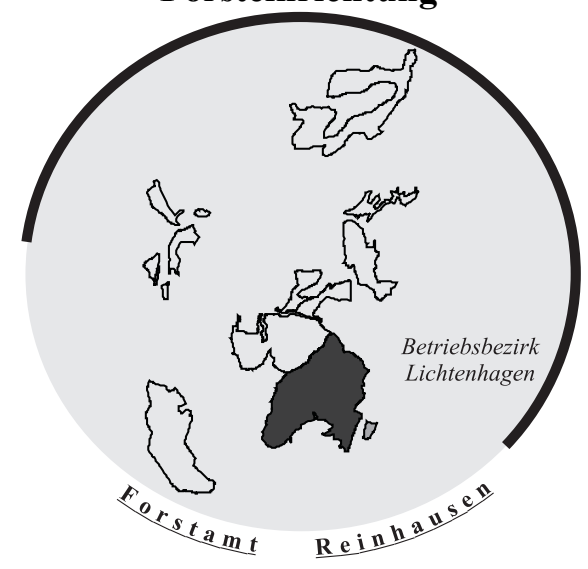

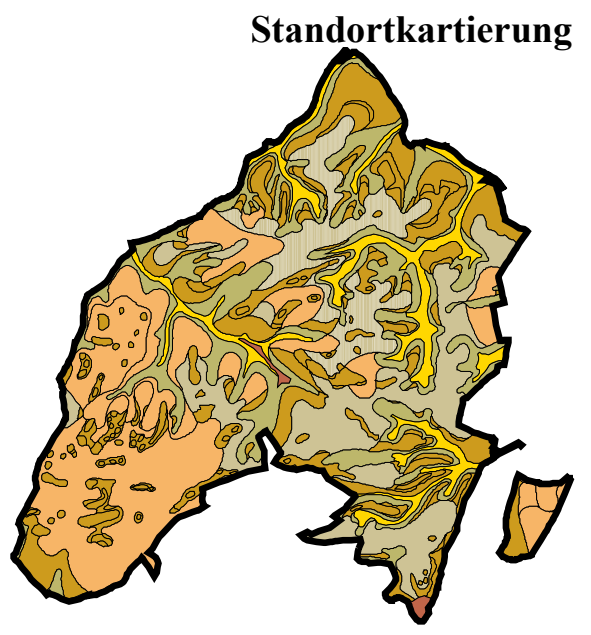

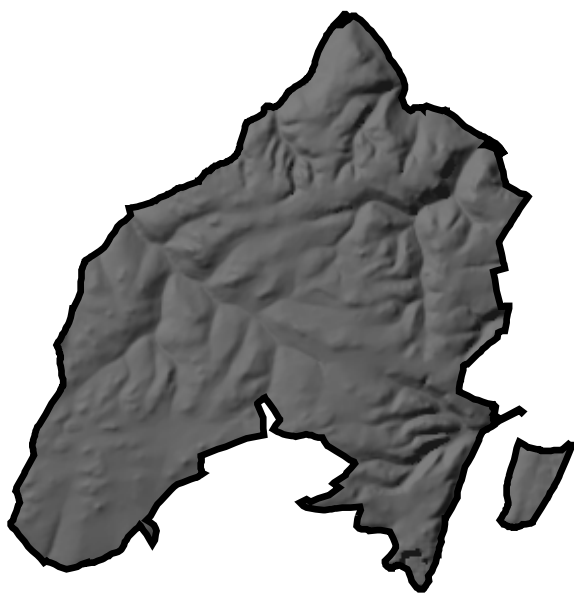

Geländemodell

Abb. 94: Die für die Fragestellung entscheidungsrelevanten Geoinformationen des Betriebsbezirks Lichtenhagen. 
Wie bereits in Kapitel 2.4 gezeigt wurde, handelte sich in der ursprünglichen Fragestellung nicht explizit um ein Entscheidungsproblem, sondern um eine bedingte räumliche Suche nach geeigneten Einsatzorten. Die Berücksichtigung mehrerer, teilweise konfliktärer Kriterien sowie die Beachtung von Kompensationsmöglichkeiten einer schlechten Ausprägung eines Kriteriums durch eine bessere Ausprägung eines anderen Kriteriums, lässt die Komplexität einer solchen Fragestellung wesentlich ansteigen.

Das Beispiel wurde, wie in SPORS et al. (1992), für den Betriebsbezirk Lichtenhagen des Niedersächsischen Staatlichen Forstamtes Reinhausen realisiert (Abbildung 94). Er befindet sich im Wuchsbezirk Göttinger Wald, Wuchsgebiet Südniedersächsisches Bergland. Seine Fläche umfasst 625 ha. Die im Beispiel verwendeten Geodaten (Abbildung 94) gehören zum Datenbestand des Instituts für Forstliche Biometrie und Informatik der Universität Göttingen. Die Daten der Forsteinrichtung stammen aus dem Jahr 1991, die der Standortskartierung von 1967. Beide wurden vom Niedersächsischen Forstplanungsamt mit Sitz in Wolfenbüttel bezogen. Das Digitale Geländemodell (DGM) wurde vom Amt für Landvermessung und Geobasisinformation Niedersachsen (LGN) 1992 erstellt.

\subsection{Struktur der Entscheidungssituation}

Das Ziel der anstehenden Entscheidung ist es, geeignete Einsatzorte der mechanisierten Arbeitsausführung (Harvester) bei der Nadelholzdurchforstung im Betriebsbezirk Lichtenhagen abzugrenzen. Die zu berücksichtigenden Kriterien des Entscheidungsträgers sind schematisch in der Abbildung 95 dargestellt.

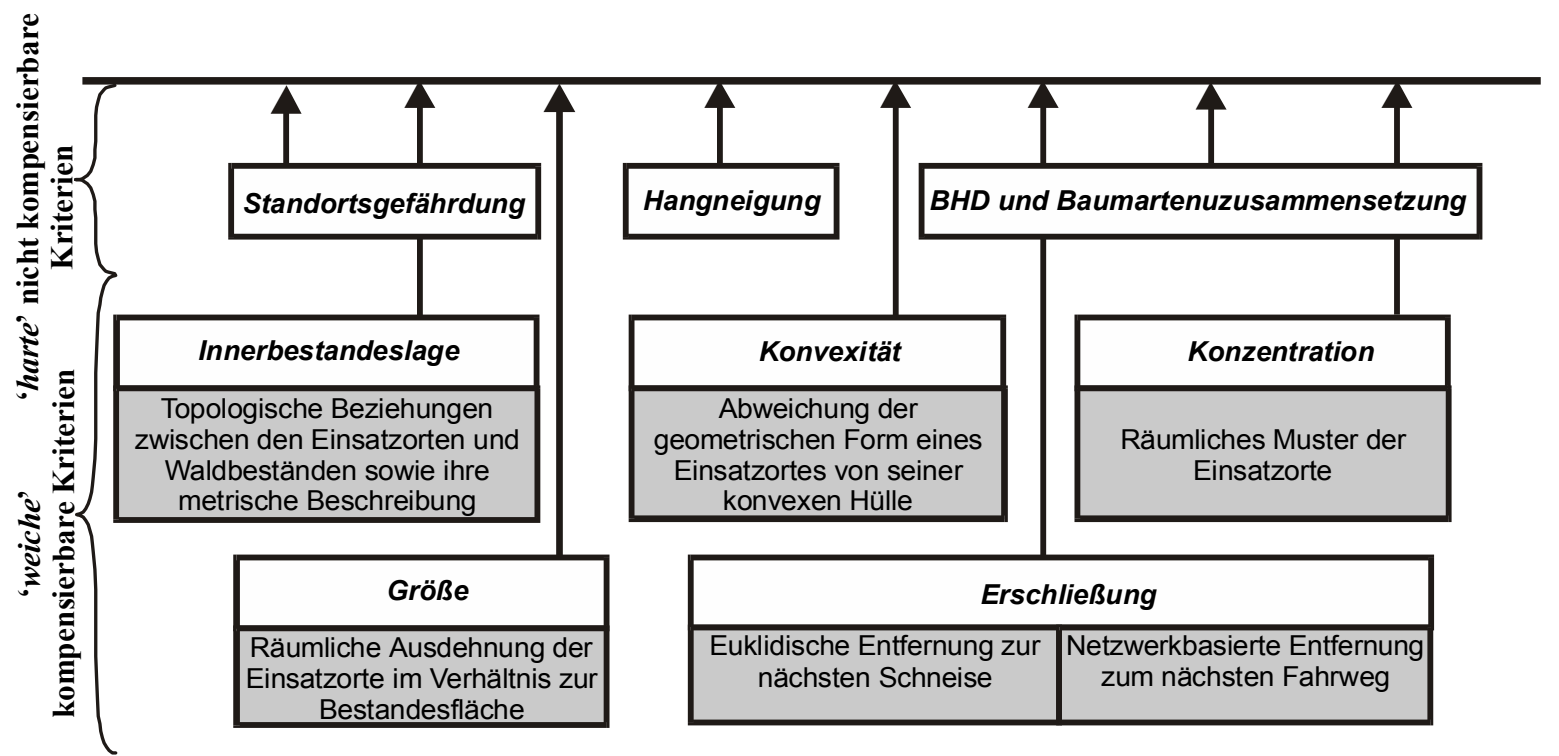

Abb. 95: Die bei der Suche nach geeigneten Einsatzorten für den Harvester zu berücksichtigenden Entscheidungskriterien. Erweiterung gegenüber der Fragestellung in SPORS et al. (1992) um die kompensierbaren Kriterien.

Die ursprünglichen Auswahlkriterien nach SPORS et al. (1992) werden als ,harte' Bedingungen (constraints) angenommen, die keine Kompensation zulassen. Diese werden um weitere, sog. 
,weiche' Kriterien ergänzt, welche die Vorteilhaftigkeit des Harvestereinsatzes in konkreten räumlichen Situationen detaillierter beschreiben (vgl. Kap. 7.3). Während die ,harten' Kriterien zum Ausschluß der für den Harvestereinsatz nicht geeigneten Flächen bestimmt sind, dienen die ,weichen' Kriterien zur Abgrenzung der vom Entscheidungsträger bevorzugten Einsatzorte.

In der Entscheidungssituation werden nur zwei Alternativen berücksichtigt: Es soll darüber entschieden werden, ob in einer räumlichen Bezugseinheit der Harvester eingesetzt werden soll oder nicht. Danach sollen der ersten Alternative alle Flächen zugeordnet werden, auf welchen der Harvestereinsatz möglich ist und gleichzeitig auch als vorteilhaft angesehen wird. Der zweiten Alternative sollen dann die Flächen zugewiesen werden, an welchen der Harvestereinsatz möglich aber nicht vorteilhaft ist.

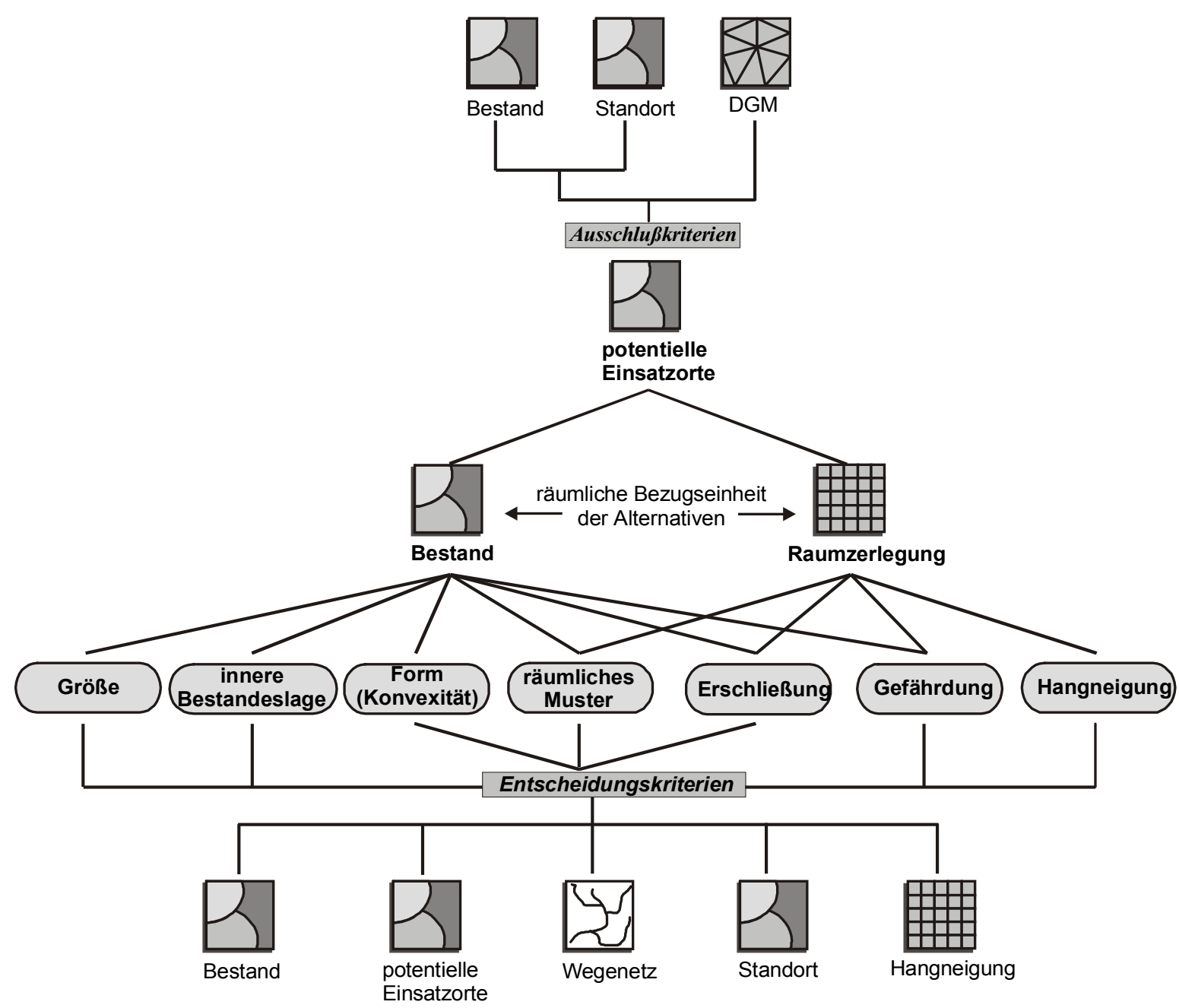

Abb. 96: Semantischer Aufbau der Entscheidungssituation und Ableitung der Entscheidungskriterien.

Der räumliche Bezug der Alternativen wird sowohl für den Bestand als auch für die Zelle einer Raumzerlegung modelliert. Im ersten Fall dient der Bestand als Bezugseinheit und Träger der entscheidungsrelevanten Kriterienausprägungen der Alternativen. Jede räumlich - zusammenhängende Einsatzfläche (potentieller Einsatzort) innerhalb eines Bestandes wird als eine Alternative behandelt. Im zweiten Fall wird eine Zelle als Bezugseinheit der Alternativen angenommen. Der Entscheidungsraum (die räumliche Ausdehnung der potentiellen Einsatzorte, siehe Abbildung 97) wird durch eine Partition in Zellen zerlegt. In jeder Zelle wird 
der Harvestereinsatz präferenzorientiert beurteilt. Dabei können die Kriterien, welche sich auf eine qualitative räumliche Beziehung räumlich ausgedehnter Objekte stützen (z.B. die topologischen Beziehungen des Bestandes und Einsatzortes), nicht berücksichtigt werden.

Alle modellierten Entscheidungskriterien beschreiben die Vorteilhaftigkeit der räumlichen Bezugseinheit für den Harvestereinsatz. Die Entscheidung soll in Form einer Mindestgrenze (eines Anspruchsniveaus) getroffen werden, die bei der ganzheitlichen Betrachtung der aggregierten Präferenzwerte und einer interaktiven Überprüfung durch den Entscheidungsträger angegeben wird. Für die einzelnen Entscheidungskriterien wird die Höhenpräferenz (Wertfunktion, Kap. 6.6) abgeleitet. Diese werden dann mit Hilfe der Artenpräferenz miteinander verknüpft (Kap. 6.6.2). Der Schwellenwert der Gesamtpräferenz als gesuchte Entscheidung kann dann interaktiv gefunden werden.

\subsubsection{Entscheidungskriterien}

\subsubsection{Nicht kompensierbare Bedingungen}

Der Harvestereinsatz wird für Nadelholzrein- und -mischbestände ab zweiter Durchforstung mit einer BHD-Spanne von $12-35 \mathrm{~cm}$ vorgesehen (Abbildung 97). Als obere Grenze der verträglichen Hangneigung beim Harvestereinsatz wird 30\% angenommen.

Aus bodenökologischen Gründen wird der Harvestereinsatz auf Standorte mit geringer Gefährdung eingeschränkt. Die Standortseigenschaften Wasserhaushalt und Substrat wurden in dem ursprünglichen Beispiel zur Ausscheidung von Gefährdungsstufen (Standortsgefährdung) angewendet (Tab. 29). In SPORS et al. (1992) werden die möglichen Kombinationen der Gefährdungsstufen nach einzelnen Standortseigenschaften (I/II, I/III, I/IV ...., IV/III, IV/IV) außer Acht gelassen. Im weiteren werden diese Kombinationen als kompensierbare Entscheidungskriterien angewendet. Für das Beispiel wurden als Ausschlußbedingungen für den Harvestereinsatz die Gefährdung nach Wasserhaushalt I - II und nach Substrat I angenommen.

Tab. 29: Klassifizierungsregel zur Ausscheidung von Gefährdungsstufen von Waldstandorten (nach SPORS et al. 1992).

\begin{tabular}{|c|c|c|c|c|}
\hline Standortstyp & $\begin{array}{l}\text { Stufe I } \\
\text { hochgradig gefährdet }\end{array}$ & \begin{tabular}{|l} 
Stufe II \\
stark gefährdet
\end{tabular} & \begin{tabular}{|l} 
Stufe III \\
mäßig gefährdet
\end{tabular} & \begin{tabular}{|l} 
Stufe IV \\
gering gefährdet
\end{tabular} \\
\hline $\begin{array}{l}\text { nach } \\
\text { Wasserhaushalt }\end{array}$ & $\begin{array}{l}1,2,3 \text { Schluchten } \\
5 \text { nasse Tal- und } \\
\text { Grundwasserstandorte } \\
6 \text { Quellbereiche } \\
\text { 15,16 Naßgley u. Moore } \\
\text { 28, 29 Steilabstürze }\end{array}$ & $\begin{array}{l}4 \text { V-Täler } \\
7 \text { frische Talstandorte } \\
12 \text { wechseltrocken } \\
13 \text { Stauwasserstandorte, } \\
\text { naß } \\
17,21 \text { feuchte Hanglagen }\end{array}$ & $\begin{array}{l}8 \text { grundwasserferne Täler } \\
18,22 \text { nachhaltig frische } \\
\text { Hanglagen } \\
\text { 14 Stauwasserstandorte, } \\
\text { frisch }\end{array}$ & $\begin{array}{l}\text { alle nicht } \\
\text { genannten } \\
\text { Schlüsselziffern }\end{array}$ \\
\hline nach Substraten & $16 \mathrm{I}-\mathrm{V}$ Moore & $\begin{array}{l}\text { 3.1, 3.2, 3.3, 3.4 Lösse } \\
\text { 4.3, 4.4, Lehme üb. Kalk } \\
\text { 7.1, 7.2, 7.3, 7.4 Alluvien } \\
\text { 5.1, 5.2 hochanstehende } \\
\text { Tone }\end{array}$ & $\begin{array}{l}\text { 2.3, 2.4, verlehmte arme } \\
\text { Silikatb. } \\
\text { 5.3 Tone } \\
6.3,6.4 \text { verlehmte reiche } \\
\text { Silikatb. }\end{array}$ & $\begin{array}{l}\text { alle nicht } \\
\text { genannten } \\
\text { Schlüsselziffern }\end{array}$ \\
\hline
\end{tabular}

In der Abbildung 97 sind die Ausschlußbedingungen des Harvestereinsatzes räumlich umgesetzt. Die einzelnen Ausschlußkarten (constraints map) werden anschließend verknüpft und potentielle Einsatzorte für den Harvester abgeleitet. Die Karten der potentiellen Einsatzorte in der Abbildung 97 zeigen sowohl die Bestände in welchen der Harvester eingesetzt werden kann, als auch die 
möglichen Einsatzflächen innerhalb der Bestände. Die Aufgabe der Entscheidungsfindung ist es, die Bestände sowie die Einsatzorte entsprechend der Präferenz des Entscheidungsträgers abzugrenzen.

Abb. 97: Ableitung potentieller Einsatzorte: Die nicht-kompensierbaren Kriterien (constraints) mit ihrer räumlichen Umsetzung (oben), die durch Verschneidung der constraints entstandenen potentiellen Einsatzorte.

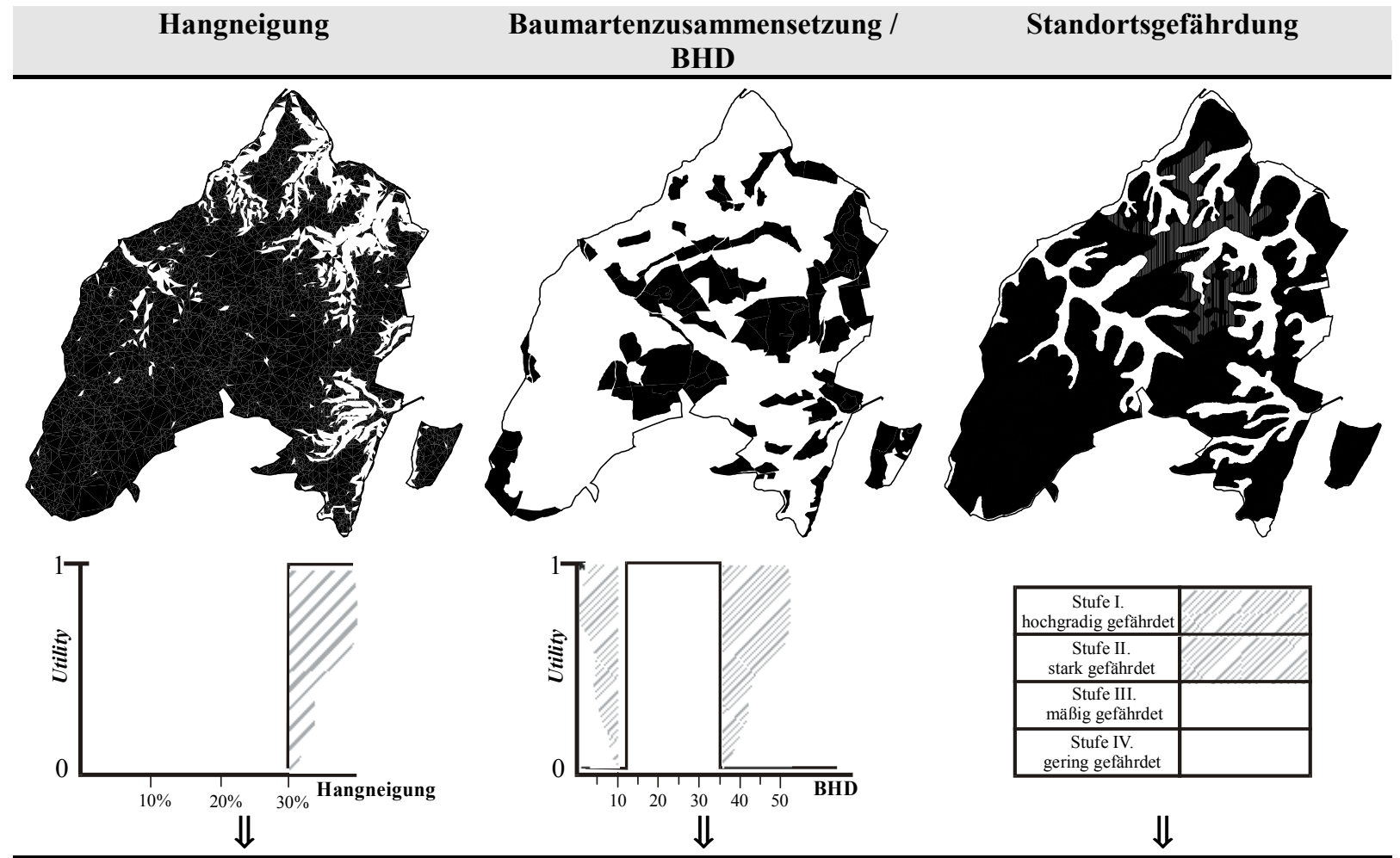

\begin{tabular}{ccc}
\hline & potentielle Einsatzorte des Harvesters \\
\hline Bestände, in welchen & Ausdehnung möglicher Einsatzorte & $\begin{array}{c}\text { Gegenüberstellung von beiden } \\
\text { räumlichen Bezugseinheiten }\end{array}$ \\
Harvestereinsatz möglich ist. & innerhalb des Bestandes & .
\end{tabular}

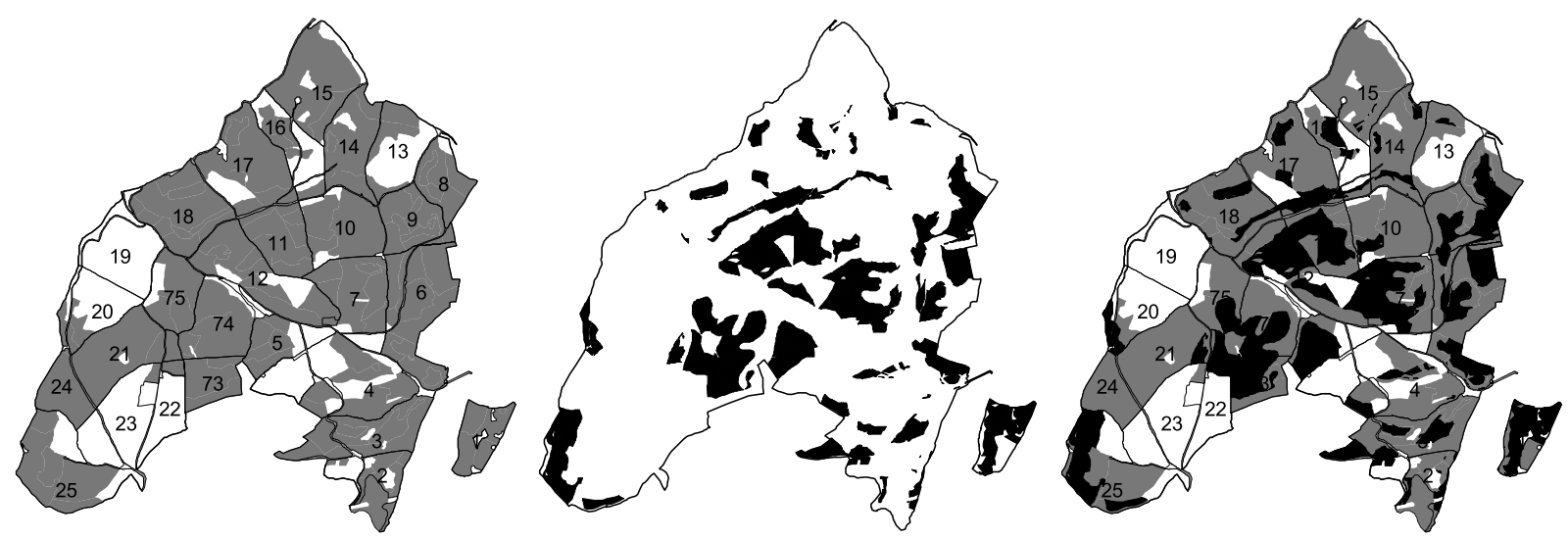

Einsatzort $\square$ Bestand

Maßstab 1: 60000 


\subsubsection{Entscheidungskriterien}

\section{Innerbestandeslage}

Jede der möglichen topologischen Beziehungen der Inhaltsinvariante in $\mathbb{R}^{2}$ kann die relative räumliche Lage der Einsatzorte zu den Beständen beschreiben. Als vorteilhafter werden jedoch Einsatzorte (E) angesehen, welche eine nicht-leere Schnittmenge mit dem Rand eines Bestandes ( $\partial \mathrm{B})$ haben: $\mathrm{E} \cap \partial \mathrm{B} \neq \varnothing \succ \mathrm{E} \cap \partial \mathrm{B}=\varnothing$. Dieses Kriterium ermöglicht es die von den unbedeutenden \{meet, disjoint\} Beziehungen verschiedenen topologischen Beziehungen in drei Klassen einzuteilen, welche sich in ihrer Präferenz unterscheiden (Tab. 30). Die erste Beziehungsklasse \{equal, contains\} beschreibt den Fall des vorteilhaftesten Einsatzes, die dritte Klasse $\{i n\}$ den ungünstigsten Fall des Einsatzes. Die letzteren zwei Klassen lassen sich mit Hilfe metrischer Konzepte detaillierter beschreiben (siehe Kap. 5.3.2.8).

Tab. 30: Die entscheidungsrelevanten topologischen Beziehungen der Einsatzorte und Bestände (nach der Inhaltsinvariante) und ihre mögliche Quantifikation.

\begin{tabular}{c|c|c|c}
\hline Bestand & $\begin{array}{c}\text { Equal } \\
\text { contains }\end{array}$ & $\begin{array}{c}\text { covers } \\
\text { covered By } \\
\text { overlap }\end{array}$ \\
\hline Quantifikation & - & $\begin{array}{c}\text { area splitting } \\
\text { closeness }\end{array}$ \\
$\begin{array}{c}\text { Präferenz- und } \\
\text { Indifferenz- } \\
\text { Relationen }\end{array}$ & Indifferent
\end{tabular}

\section{Konvexität}

Konvexität bezeichnet eine geometrische Eigenschaft eines räumlichen Objektes. Eine Menge $C$ in $\mathbb{R}^{n}$ heißt konvex, wenn für jedes Punktepaar $p_{1}, p_{2}$ der Menge $C$ auch die Strecke zwischen $p_{1}$ und $p_{2}$ in $C$ enthalten ist. Die konvexe Hülle einer Punktmenge $S$ in $\mathbb{R}^{n}$ ist der Abschluß der kleinsten konvexen Menge in $\mathbb{R}^{n}$ die $S$ enthält (Abbildung 98).

Die entscheidungsrelevante Interpretation dieses Kriteriums ist die notwendige Mehrfahrt des Harvesters in den gleichen Bestandesteilen. Die Konvexität der Einsatzorte wurde mit Abweichung der Flächen der Einsatzorte zu ihrer konvexen Hülle quantifiziert (Abbildung 98). Eine Alternative ist die Quantifikation von Inkonvexität durch den Index Quiescence (SCHNEIDER R. 1993), welcher rechnerisch allerdings sehr aufwendig ist. 

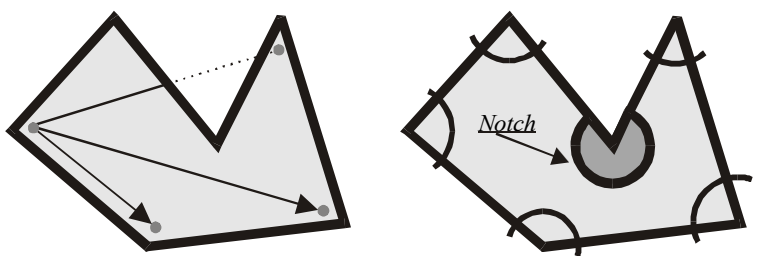

A)
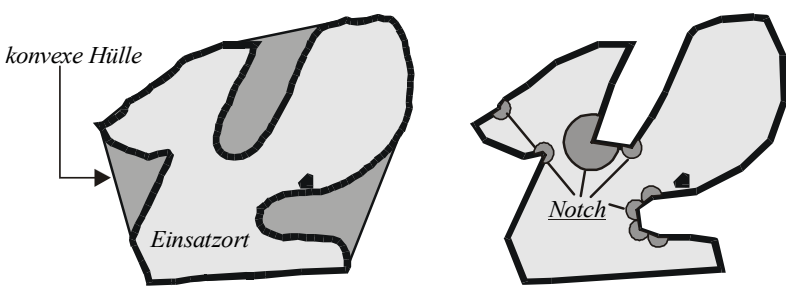

B)

Abb. 98: Konzepte der Konvexität räumlicher Objekte: (A) Konzept der Konvexität und des Notches; (B) konvexe Hülle und Beschreibung der Inkonvexität eines potentiellen Einsatzortes.

Der Index Quiescence quantifiziert die Abweichung eines Polygons von seiner konvexen Hülle. Er setzt sich aus drei Einzelparametern: Dither, Umfangsabnahme und Flächendifferenz zusammen, die zu einer einzigen Kennzahl komprimiert werden:

Quiescence $=\frac{(8 \times(a \times b)+2 \times c)}{10}$

Umfangsabnahme: $a=\frac{(\text { Umfang }(\text { Einsatzort })-U m f a n g(\text { konvexeHülle }))}{U m f a n g(\text { Einsatzort })}$

Dither: $\quad b=f($ Notchesnorm $)$

Flächendifferenz: $c=\frac{(\text { Fläche }(\text { konvexeHülle })-\text { Fläche }(\text { Einsatzort }))}{\text { Fläche }(\text { konvexeHülle })}$

Die Zittrigkeit (Dither) ist eine Funktion der Notchesnorm. Ein Notch (Abbildung 98) beschreibt eine Stelle der Inkonvexität eines Polygons. Es liegt dann vor, wenn der Innenwinkel zwischen zwei aneinanderfolgenden Kanten größer ist als $180^{\circ}$. Ein Polygon ohne Notches ist ein konvexes Polygon. Ein n-Eck kann höchstens n-3 Notches haben. Die Anzahl der Notches kann durch Division mit (n-3) auf den Bereich [0,1] normiert werden. Liegt der Wert der Notchesnorm nahe 0 , ist das Objekt ausgeprägt konvex. Geht der Wert gegen 1, zeichnet es sich durch eine starke Konkavität aus. In beiden Fällen ist die Zittrigkeit des Polygons als gering einzustufen. Die maximale Anzahl der Richtungsänderungen ist dann vorhanden, wenn sich eine Konkavität regelmäßig mit einer Konvexität abwechselt.

\section{Größe}

Die Größe des Einsatzortes, sowohl in ihrer absoluten als auch ihrer relativen auf die Bestandesfläche bezogenen Form, ist für die Vorteilhaftigkeit des Einsatzes von großer Bedeutung. Je kleiner die Einsatzfläche, desto unvorteilhafter erscheint der Einsatz des Harvesters. Darüber hinaus wird es als vorteilhafter empfunden, wenn der ganze Bestand mit gleicher Arbeitsausführung (Harvester, motormanuelle Alternative) bei der Durchforstung behandelt wird. Desgleichen werden größere Einsatzflächen innerhalb eines Bestandes als vorteilhafter angesehen.

\section{Erschließung}

Die Erschließung ist u.a. ein Maß für den Transportaufwand des Harvesters zu dem Ort seines Einsatzes. Das Maß stützt sich auf das zugrundeliegende räumliche Konzept der Distanz. Sie besteht aus zwei Komponenten, die jeweils auf einer anderen Metrik basieren (vgl. Kap. 5.3.3). 
Die äußere Erschließung bezeichnet in diesem Beispiel die kürzeste netzwerkbasierte Entfernung zum nächsten Fahrweg, die Entfernung also, welche der Harvester auf dem Rückeweg zurücklegen muß, um vom Fahrweg zum Einsatzort zu gelangen. Die innere Erschließung bezeichnet wiederum die kürzeste euklidische Entfernung des Einsatzortes zu dem Rückeweg mit kleinster Netzwerkentfernung zum Fahrweg (Abbildung 99).
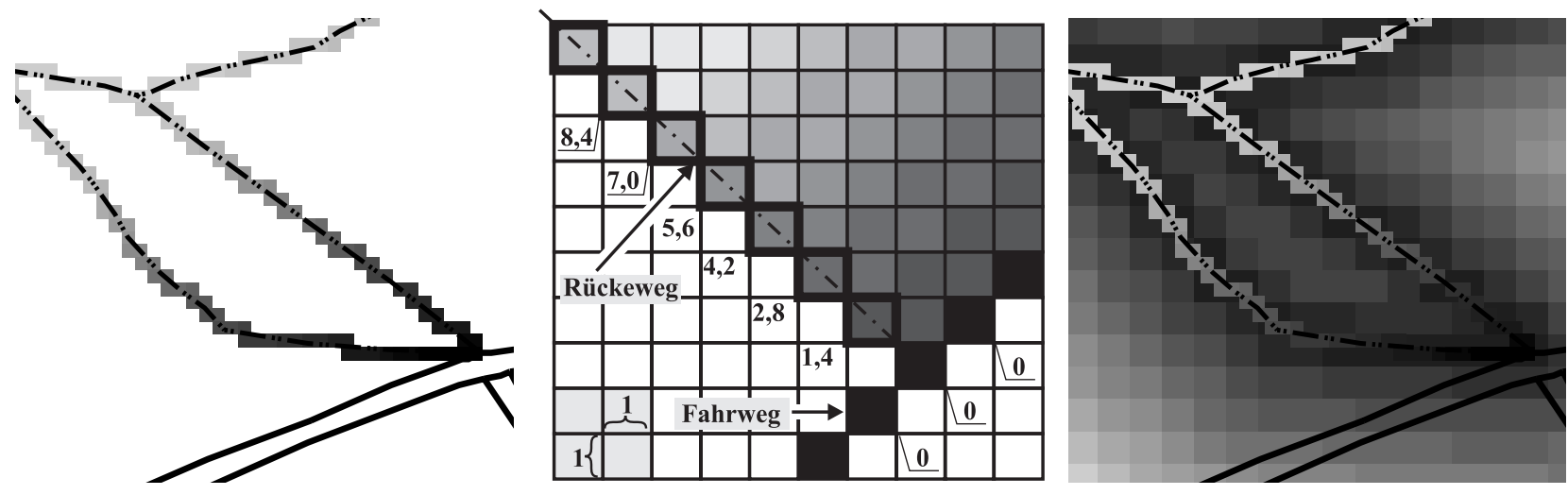

Abb. 99: Konzeption der Erschließung von Einsatzorten: (links) netzwerkbasierte äußere Erschließung, (mitte) schematische Darstellung der Erschließungskonzepte, (rechts) räumliche Darstellung beider Konzepte.

Für das Beispiel werden beide Erschließungsdistanzen berücksichtigt. Bei der Bestimmung der netzwerkbasierten Entfernung zum nächsten Fahrweg werden nur solche Rückewege berücksichtigt, welche für den Harvester aufgrund der Hangneigung keine unpassierbaren Stellen aufweisen. Aufgrund der Unterbrechung einiger Rückewege durch solche Stellen werden aus der Berechnung auch alle Rückewege ohne einen Anschluss an einen Fahrweg ausgeschlossen.

\section{Konzentration}

Als entscheidungsrelevante Eigenschaft der räumlichen Verteilung von Einsatzorten wird ihre gegenseitige euklidische Entfernung angenommen. Über die Vorteilhaftigkeit eines einzelnen Einsatzortes hinaus ist die Konzentration von Einsatzorten für die Entscheidungsfindung von Bedeutung. Eine größere Konzentration auch solcher Einsatzorte, die nach eigenen Eigenschaften für den Einsatz des Harvesters eher ungünstig ausfallen, wird der Entscheidungsträger positiver bewerten als solche, die vereinzelt in ihrer nahen Umgebung vorkommen. Desgleichen wird der Entscheidungsträger die Nähe zu einem einzigen Einsatzort mit großem Präferenzwert für den Harvestereinsatz als vorteilhaft einstufen (Abbildung 100).

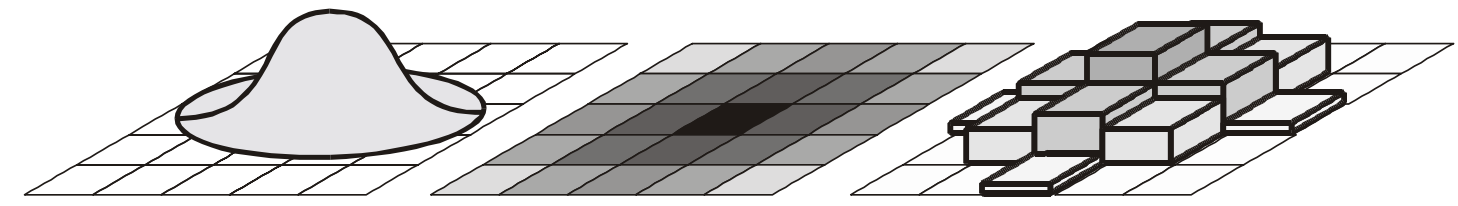

Abb. 100: Konzept der Konzentration von Einsatzorten als Dispersion ihrer Präferenzen für den Einsatz eines Harvesters: (links) die Funktion der Präferenzdispersion und (mitte, rechts) ihre zellenbasierte Umsetzung.

In der Abbildung 100 ist das Konzept der Konzentration von Präferenzen der potentiellen Einsatzorte als (kumulative) Dispersion der Präferenzwerte dargestellt. Von jedem potentiellen Einsatzort aus wird seine Präferenz als Funktion der euklidischen Entfernung modelliert. Diese kann bei der Bewertung von anderen Einsatzorten mitberücksichtigt werden. 


\section{Baumartenzusammensetzung}

Im zulässigen Bereich der Baumartenzusammensetzung (Nadelholzrein- und -mischbestände) wird die Vorteilhaftigkeit des Einsatzes durch den perzentualen Anteil der Nadelholzarten detaillierter beschrieben.

\section{Hangneigung}

Im Bereich der zulässigen Hangneigung $(<30 \%)$ wird ein Intervall ausgeschieden, in welchem der Entscheidungsträger aus Sicherheitsgründen den Einsatz des Harvesters als weniger vorteilhaft ansieht. Für das Beispiel wurde das Intervall $(20,30)$ definiert. Um den Harvester in diesem Intervall einzusetzen, müssen die Ausprägungen in anderen Entscheidungskriterien besser ausfallen.

\section{Standortsgefährdung}

Das Entscheidungskriterium Standortsgefährdung wird in seinen Ausprägungen getrennt nach seinen Komponenten betrachtet. Dies erlaubt auch eine transparente Behandlung solcher Fälle, in welchen sich die Gefährdung des Standortes nach dem Wasserhaushalt und dem Substrat unterscheiden (z.B. Gefährdung nach dem Wasserhaushalt III und nach dem Substrat IV).

\subsubsection{Bestimmung der Wertfunktion}

Die Entscheidungsfindung soll dem Entscheidungsträger die Abbildung seiner Präferenz in Einzelwertfunktionen erleichtern. Zur Lösung dieser Aufgabe wurden in der Entscheidungsforschung mehrere Ansätze entwickelt, bei welchen dem Entscheidungsträger in einer Befragung einfache Wertvergleiche vorgegeben werden, zwischen welchen er eine Indifferenz- oder Präferenzaussage zu treffen hat: direct-rating Methode, Methode der gleichen Wertdifferenzen oder Halbierungsmethode. Die Methoden der Wertfunktionsbestimmung sind weitgehend in der Literatur beschrieben (EISENFUEHR \& WEBER 1993, LAUX 1998, SCHNEEWEISS 1991). Da sie für das Beispiel nicht von großer Bedeutung sind, wird im weiteren auf eine detaillierte Beschreibung verzichtet.
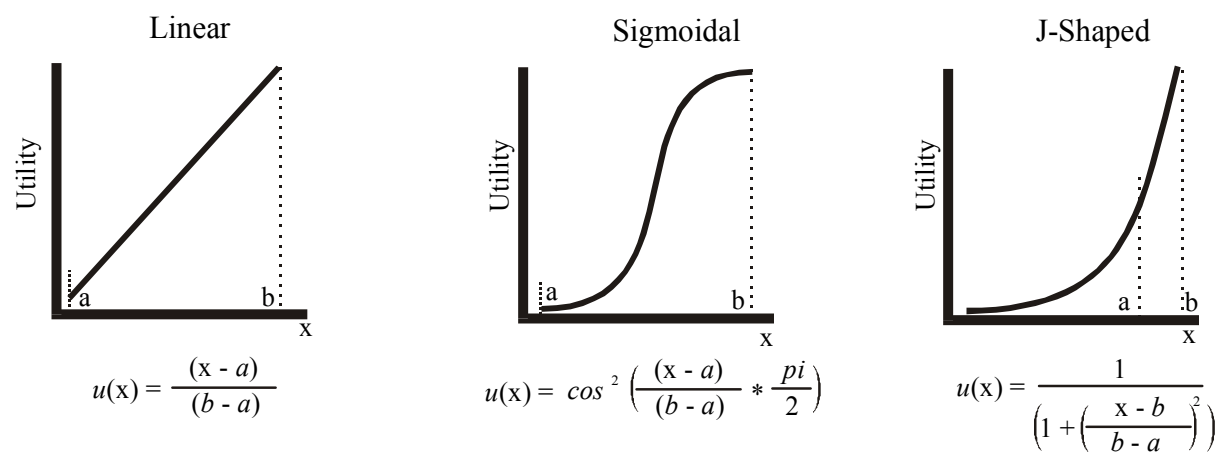

Abb. 101: Unterschiedliche Typen der Wertfunktion zur Abbildung der Präferenzen (Nutzen, utility) hinsichtlich der Konsequenzen der Alternativen (Ausprägungen der Entscheidungskriterien).

Die abzuleitende Wertfunktion wird in einem Diagramm dargestellt, das auf der Abszissenachse die Ausprägungen der Entscheidungskriterien (Konsequenzen der Alternativen) und auf der Ordinatenachse die Werte der Wertfunktion, üblicherweise auf das Intervall [0,1] normiert, enthält. In der Abbildung 101 sind drei Typen der Wertfunktionen dargestellt. 
Abb. 102: Kompensierbare, weiche' Entscheidungskriterien (factors) und ihre räumliche Umsetzung.

\section{modellierte \\ Wertfunktion}

Übersicht $(1: 60$ 000) der umgesetzten Kriterien mit den bestandesbezogenenen potenziellen Einsatzorten als räumliche Bezugseinheit
Detailansicht (1:18 000) der umgesetzten Kriterien bezogen auf die Zellen der Raumzerlegung als räumliche Bezugseinheit
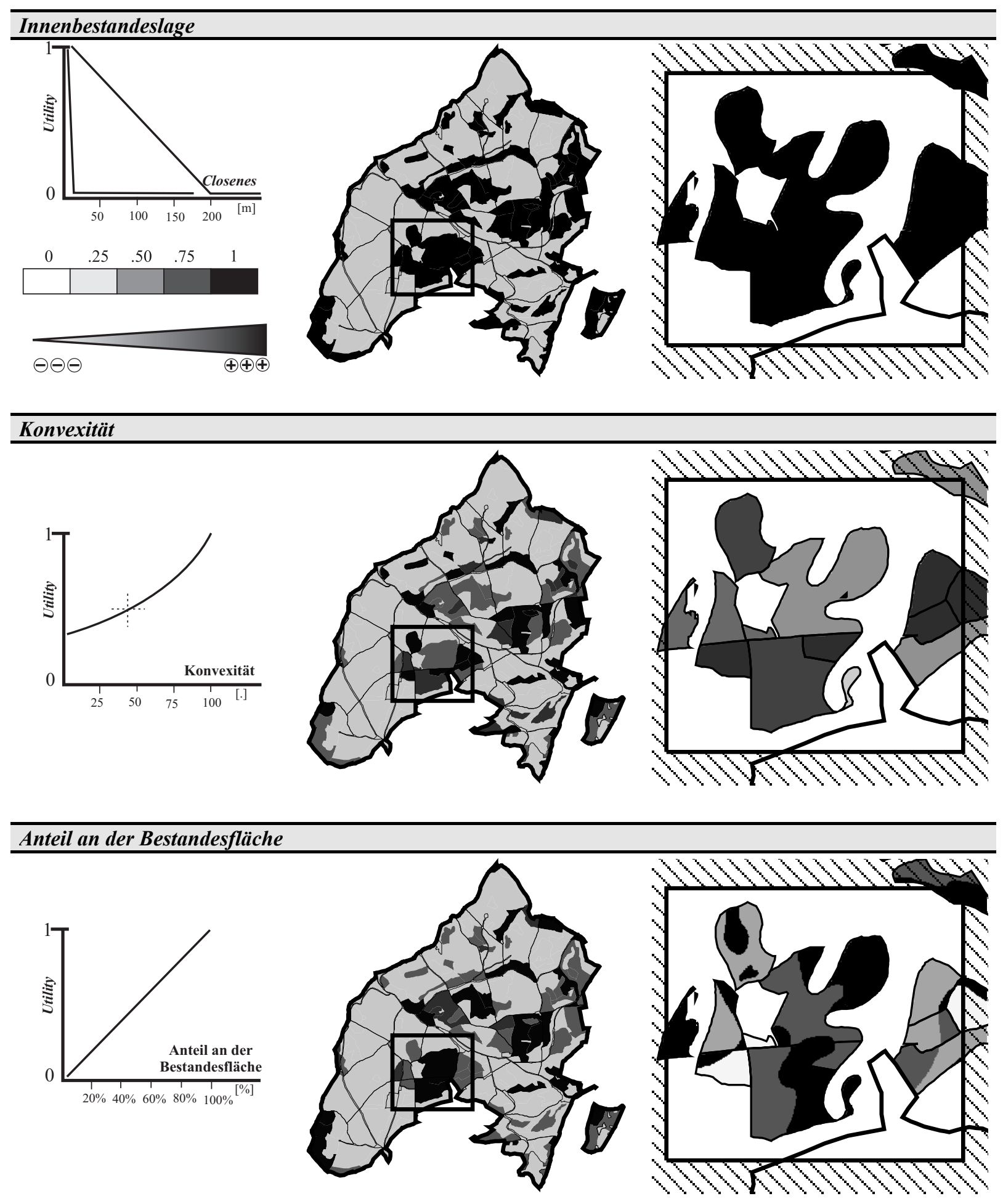

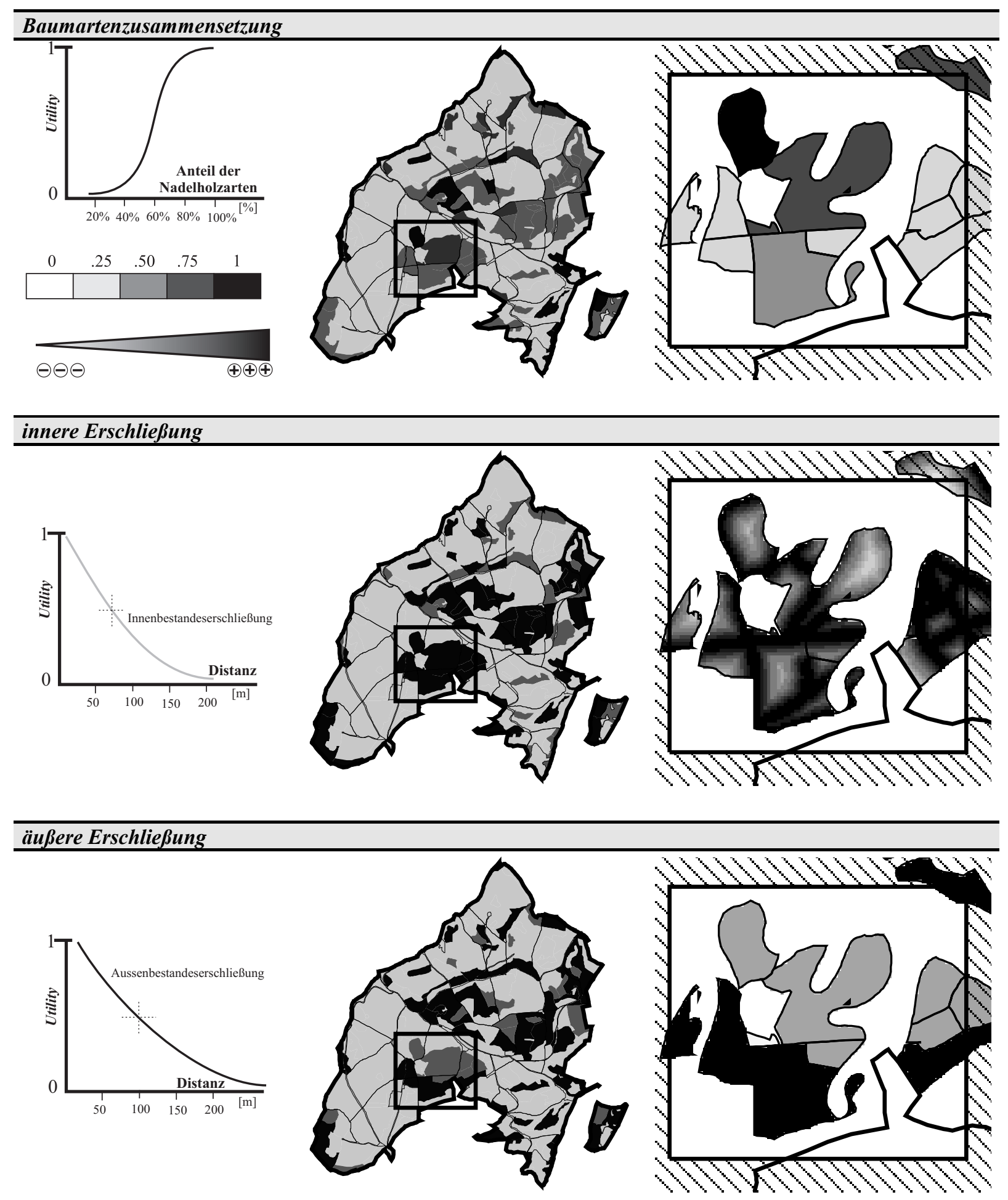

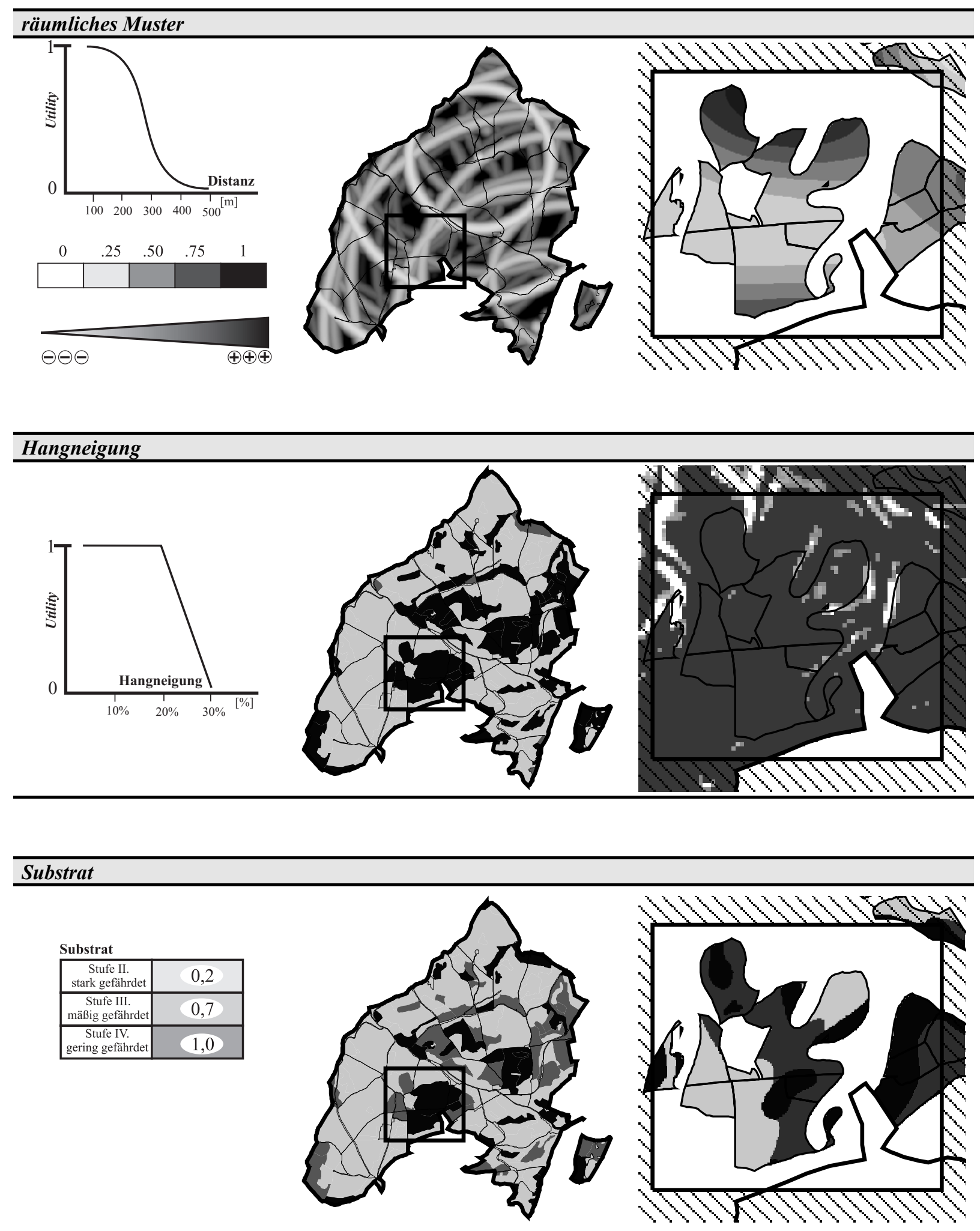


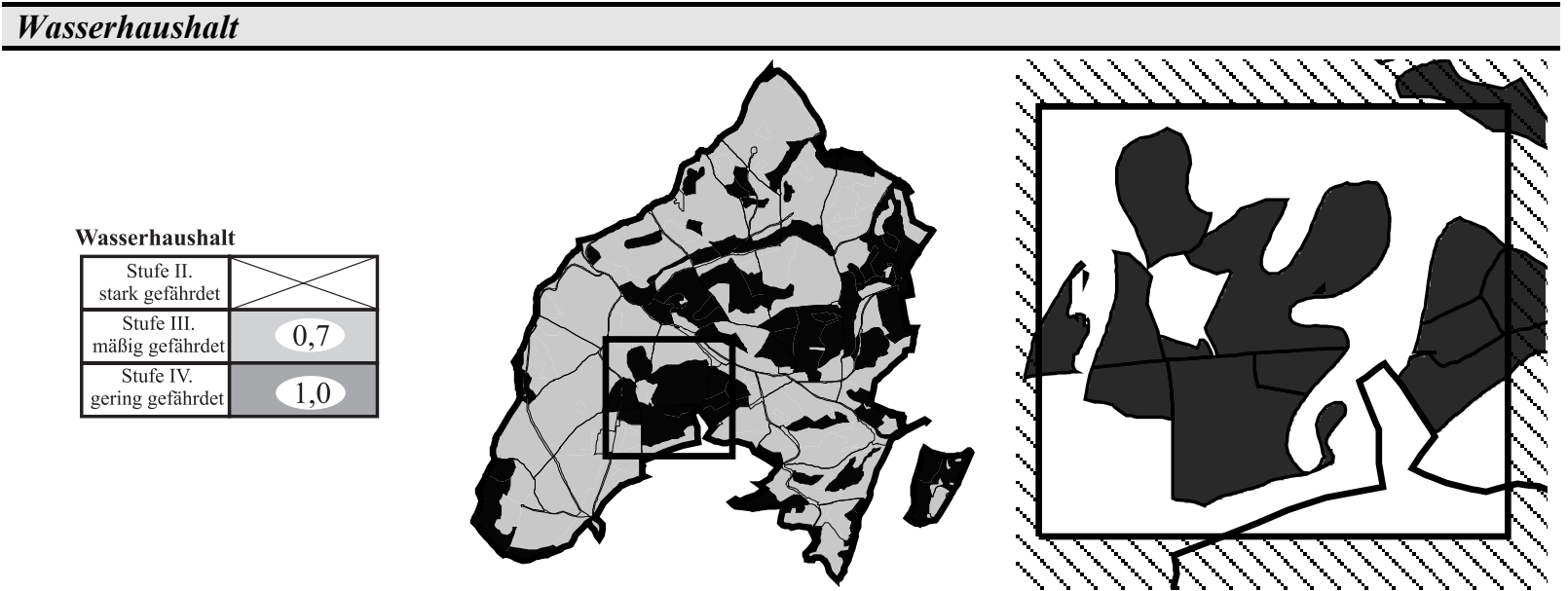

Die Umsetzung der Entscheidungskriterien ist in der Abbildung 102 dargestellt: Die Wertfunktion (v) bildet die Präferenz (Kapitel 6.6) des Entscheidungsträgers ab, seine Einsicht über die Vorteilhaftigkeit einer Alternative in bezug auf eine andere Alternative. Durch eine Abbildung der Präferenzrelationen hinsichtlich einzelner Kriterien (Alternative a wird Alternative $b$ vorgezogen) in eine numerische Beziehung v(a) $>v(b)$ ist die Aggregation mehrerer Kriterien erleichtert (vgl. Kapitel 6.6). Die Ergebnisse oder Konsequenzen der Alternativen bezüglich der einzelnen Kriterien (z.B. Hangneigung von 20\%) werden mittels Wertfunktionen in individuelle Präferenzwerte transformiert (der Hangneigung 20\% wird entsprechend der zugehörigen Wertfunktion der Präferenzwert von 1 zugeordnet). Neben der Präferenzabbildung werden die Ausprägungen der Entscheidungskriterien mittels Wertfunktionen normiert und somit miteinander vergleichbar gemacht.

Entsprechend den unterschiedlichen räumlichen Bezugseinheiten der Alternativen (Kapitel 102) wurden für die Abbildung 102 unterschiedliche Darstellungsweisen gewählt: Im ersten Fall, dargestellt im Übersichtsansicht (1: 60 000), dient jede zusammenhängende Fläche innerhalb eines Bestandes (bestandesbezogene potentielle Einsatzorte), welche den harten Kriterien genügt, als eine Alternative. Innerhalb dieser bleibt der Präferenzwert, abgebildet mit Hilfe der entsprechenden Wertfunktion, konstant. Im zweiten Fall, dargestellt im Detailansicht (1 : 18 000), bildet jede einzelne Zelle der Raumzerlegung eine Alternative. Der Präferenzwert kann auch innerhalb eines Bestandes variieren. Die beiden Fälle entsprechen der Dichotomie der konzeptionellen Raummodelle (Kapitel 3.5): Die bestandesbezogenen potentiellen Einsatzorte entsprechen dem Objektmodell (entity view), in dem eine endliche Anzahl der vorher generierten Alternativen in Betracht gezogen wird. Die zellebezogene Repräsentation der Alternativen entspricht dem Modell der kontinuierlichen Änderung (field) mit unendlich vielen Alternativen, derer Konsequenzen sich von Position zu Position ändern können.

\subsubsection{Entscheidungsregel}

Als Entscheidungsregel (siehe Kap. 6.5) für die Verknüpfung von Einzelpräferenzen werden zwei multikriterielle Entscheidungsmethoden angewendet: Nutzwertanalyse und Ordered Weighted Averaging. 
Die Nutzwertanalyse (NWA) (einfache additive Gewichtung) ist eine Klasse von Vorgehensweisen, die sich in der speziellen Art der Ermittlung von Höhen- und Artenpräferenzen unterscheiden (vgl. Kap. 6.7). Unter einem Nutzwert lässt sich "der subjektiv beeinflusste Wert einer Handlungsvariable zur Befriedigung eines definierten Bedarfs" verstehen (BIETHAN et al. 1992, S. 235). Für alle Attribute werden zuerst die Höhenpräferenzen bestimmt, indem die Wertfunktionen abgeleitet werden. Die Einzelnutzwerte werden anschließend normiert. Die Artenpräferenz wird in der Form der (normierten) 'Wichtigkeit' der Attribute durch deren Gewichte ausgedrückt. Die Wertaggregation der Höhen- und Artenpräferenz wird additiv vorgenommen.

Durch die Gewichtung wird der Einzelnutzen eines Attributes mit dem der übrigen Attribute vergleichbar. Die Bedingung der Präferenzunabhängigkeit muss erfüllt werden (vgl. Kap. 6.6). Die Quotienten der Gewichte können nicht als Substitutionsraten interpretiert werden. Die Existenz einer Substitutionsrate setzt voraus, dass der Entscheidungsträger bereit ist, die Substitution zwischen verschiedenen Attributen vorzunehmen. Das wird bei der NWA nicht vorausgesetzt. Die Substitutionsraten verlangen Wertvergleiche zwischen Ausprägungsdifferenzen verschiedener Attribute, daher müssen die Attribute mindestens auf Intervallskalenniveau messbar sein. Für die Substitution müssen kontinuierlich viele Attributsausprägungen und damit kontinuierlich viele Alternativen vorliegen (die NWA behandelt endlich viele Alternativen). Außerdem beruht die Attraktivität der NWA darauf, dass man auch ohne Substitutionsraten auskommt.

Ordered Weighted Averaging (OWA) geht auf die Arbeit von YAGER (1988) zurück. Der ursprünglich als Aggregationsoperator zweier fuzzy Zahlen entworfene Ansatz besitzt die Fähigkeit, den Durchschnitt zweier unscharfer Mengen im Intervall zwischen seinen extremen Werten (AND oder MIN) und (OR oder MAX) kontinuierlich zu kontrollieren. Durch YAGER (1988) und EASTMAN \& JIANG (1995) wurde dieser Ansatz in eine Entscheidungsregel implementiert, welche die Kompensation der Entscheidungskriterien direkt zu kontrollieren erlaubt. Neben den kriterienbezogenen Gewichten (Artenpräferenz) der Nutzwertanalyse, welche die Wichtigkeit der Kriterien miteinander vergleicht, führen die Ordnungsgewichte (order weights) einen Mechanismus ein, mit dessen Hilfe die Kriterien nicht nach ihrem qualitativen Inhalt, sondern nach der Reihenfolge ihrer Ausprägungen zusätzlich bewertet werden.
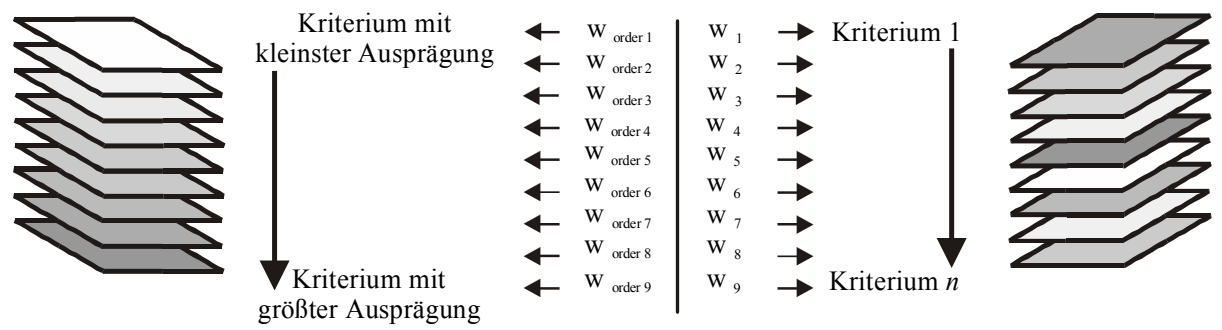

Abb. 103: Konzepte der Gewichtszuordnung bei der Nutzwertanalyse und der Ordered Weighted Averaging.

Die Ordnungsgewichte $\left(w_{\text {order }} i\right)$ sind nicht an die Kriterien gebunden, sondern an eine Position der Reihenfolge ihrer Ausprägungen. Ist beispielsweise die Reihenfolge der Ausprägungen der Kriterien $\{\mathrm{A}, \mathrm{B}, \mathrm{C}\}$ an der Position $\left\{\mathrm{x}_{1}, \mathrm{y}_{1}\right\} \quad[\mathrm{B}, \mathrm{C}, \mathrm{A}]$, dann werden die Ordnungsgewichte folgendermaßen zugeordnet: $\mathrm{B} * \mathrm{w}_{\text {order } 1}+\mathrm{C} * \mathrm{w}_{\text {order } 2}+\mathrm{A} * \mathrm{w}_{\text {order }} 3$. Ist die Reihenfolge der 
Kriterienausprägungen an der Position $\left\{\mathrm{x}_{2}, \mathrm{y}_{2}\right\}[\mathrm{C}, \mathrm{B}, \mathrm{A}]$, dann werden die Ordnungsgewichte folgendermaßen zugeordnet: $\mathrm{C} * \mathrm{w}_{\text {order } 1}+\mathrm{B} * \mathrm{w}_{\text {order } 2}+\mathrm{A} * \mathrm{w}_{\text {order } 3}$.

Die Ordnungsgewichte erlauben neben der Kompensationsmöglichkeit einer schlechteren Ausprägung eines Kriteriums durch die bessere Ausprägung eines anderen Kriteriums gleichzeitig eine Anpassung der Entscheidungsregel an das Risikoverhalten des Entscheidungsträgers. Die Ordnungsgewichte $\{1,0,0\}$ bedeuten eine Risikoaversion des Entscheidungsträgers, da dem Kriterium mit minimaler Ausprägung das gesamte Gewicht zugeordnet wird. Diese Kombination der Ordnungsgewichte entspricht der ,Maximin' Entscheidungsregel, bei welcher als optimal diejenige Alternative angesehen wird, bei der das schlechteste Ergebnis am besten ist ("Pessimisten - Regel"). Die Ordnungsgewichte $\{0,0,1\}$ ordnen das Gewicht dem Kriterium mit maximaler Ausprägung zu und entsprechen somit der ,Maximax' Entscheidungsregel, bei der wiederum diejenige Alternative als optimal angesehen wird, bei der die höchste Attributsausprägung am größten ist. In beiden Fällen werden die übrigen Attributsausprägungen vernachlässigt, sie sind durch extreme Gewichtung charakterisiert und bieten keine Möglichkeit der Kompensation. Die Ordnungsgewichte $\{0.33,0.33,0.33\}$ ordnen jeder Position der Kriterienreihenfolge das gleiche Gewicht $\mathrm{zu}$ und entsprechen somit der einfachen additiven Gewichtung. Diese Kombination der Ordnungsgewichte erlaubt die volle Kompensation der Kriterien.

Die Eigenschaften der OWA Entscheidungsregel im bezug auf das Risikoverhalten lassen sich anhand der folgenden drei Parameter beschreiben (JIANG \& EASTMAN 2000):

$$
\begin{array}{ll}
\text { ANDness }=(1 /(n-1)) \sum_{i=1}^{n}\left((n-i) W_{\text {order }}\right) & \mathrm{n} \text {..Anzahl der Kriterien } \\
\text { ORnees }=1-\text { ANDness } & \begin{array}{l}
\mathrm{i} \text {..Ordnung des Kriteriums } \\
\text { Kriteriums }
\end{array} \\
\text { TRADEOFF }=1-\sqrt{\frac{n \sum\left(W_{\text {order } i}-1 / n\right)^{2}}{n-1}} &
\end{array}
$$

Die Eigenschaft ,ANDness' beschreibt die Risikoaversion des Entscheidungsträgers, indem sie den Grad beschreibt, wie nah der Operator an der ,Minimax' Regel liegt. Die Eigenschaft ,ORness' beschreibt die Risikofreude oder den Grad, wie nah der Operator an die ,Maximax' Regel liegt.
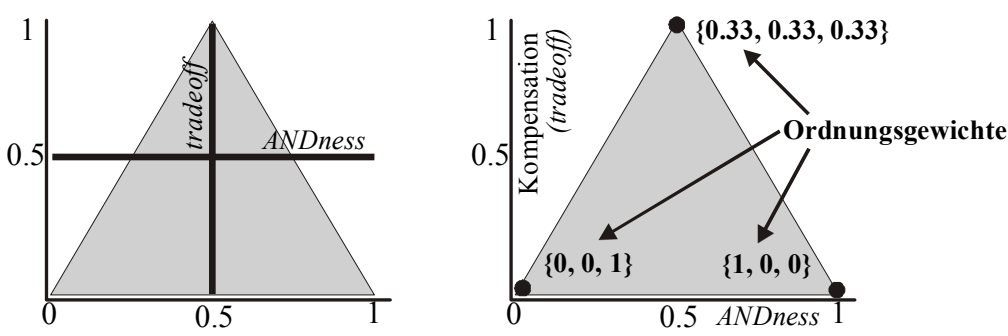

Abb. 104: Entscheidungsstrategie beim Ordered Weighted Averaging: Entscheidungsspielraum zwischen der Kompensation und der Risikofreude bzw. Riskoaversion der OWA (In Anlehnung an JIANG \& EASTMAN 2000).

Der Parameter ,Tradeoff' beschreibt die Kompensationsmöglichkeit der Kriterien. In der Abbildung 104 ist der Spielraum zwischen Risikoaversion und Risikofreude auf der einen Seite und der Kompensation auf der anderen schematisch dargestellt. 


\subsection{Ergebnisse und Diskussion}

Die Abbildung 97 zeigt die potentiellen Einsatzorte des Harvesters: Die Flächen, welche den ,harten', nicht kompensierbaren Kriterien genügen und als Entscheidungsraum für die gesuchte Lösung dienen. Bei genauer Betrachtung der entscheidungsrelevanten Kriterien soll die Präferenz des Entscheidungsträgers für den Harvestereinsatz auf diesen Flächen untersucht werden. Für die einzelnen Entscheidungskriterien werden Höhenpräferenzen abgefragt (Abbildung 102) und mit Hilfe der Entscheidungsregel (Kap. 6.5) für unterschiedliche Artenpräferenzen aggregiert.

Die Tabelle 32 enthält die in Form von Gewichten verwendeten Artenpräferenzen für beide Entscheidungsregeln. Die Nutzwertanalyse (NWA) wird sowohl für die Bestände (NWA1) als auch für die Zellen der Raumzerlegung (NWA2) als Bezugseinheiten der Alternativen angewendet. Das Ordered Weighted Averaging (OWA) aggregiert die Einzelpräferenzen für die Bezugseinheit eines Bestandes. Die Entscheidung wird als Mindestgrenze der Gesamtpräferenz angegeben.

Tab. 31: Gewichte (Artenpräferenz) die zur Aggregation der Entscheidungskriterien in den Entscheidungsregeln verwendet wurde.

\begin{tabular}{|c|c|c|c|c|c|c|}
\hline \multicolumn{3}{|c|}{ Nutzwertanalyse (NWA) } & \multicolumn{4}{|c|}{ Ordered Weighted Averaging (OWA) } \\
\hline & Kriterium & Gewicht & Kriterium & OWA1 & OWA2 & OWA3 \\
\hline \multirow{7}{*}{$\frac{\pi}{z}$} & Bestandeslage & ב.05 & 1.tes Kriterium & 0 & 1 & 2.5 \\
\hline & Konvexität & .15 & 2.tes Kriterium & 0 & 0 & 2.1 \\
\hline & Bestandesfläche & .25 & 3.tes Kriterium & 0 & 0 & 1.8 \\
\hline & Innere Erschließung & .05 & 4.tes Kriterium & 0 & 0 & 1.4 \\
\hline & Äußere Erschließung & .2 & 5.tes Kriterium & 0 & 0 & 1.1 \\
\hline & Baumartenzusammensetzung & .1 & 6.tes Kriterium & 0 & 0 & .7 \\
\hline & Dispersion & .2 & 7.tes Kriterium & 1 & 0 & .4 \\
\hline \multirow{4}{*}{$\sum_{z}^{N}$} & \multirow{4}{*}{$\begin{array}{l}\text { Wasserhaushalt } \\
\text { Substrat } \\
\text { Innere Erschließung } \\
\text { Hangneigung } \\
\text { Konzentration }\end{array}$} & \multirow{4}{*}{$\begin{array}{l}.2 \\
.2 \\
.1 \\
.1 \\
.4\end{array}$} & ANDness & 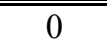 & 1 & .663 \\
\hline & & & ORness & 1 & 0 & .337 \\
\hline & & & Tradeoff & 0 & 0 & .799 \\
\hline & & & & & & \\
\hline
\end{tabular}


Abb.105: Ergebnisse der Nutzwertanalyse (Modelle NWA1 und NWA2 nach der Tabelle 32) mit Schwellenwerten $[0.3,0.5,0.7]$. Alle Einsatzorte, deren Nutzenwert größer ist als der Schwellenwert (Mindestwert), werden für den Harvestereinsatz vorgesehen. Die schwarzen Flächen zeigen die Flächen, wo der Harvestereinsatz nach den modellierten Präferenzen als vorteilhaft angesehen wird.

\begin{tabular}{ccc}
\hline & NWA1 & \\
\hline$>.3$ & $>.5$ & $>.7$ \\
\hline
\end{tabular}
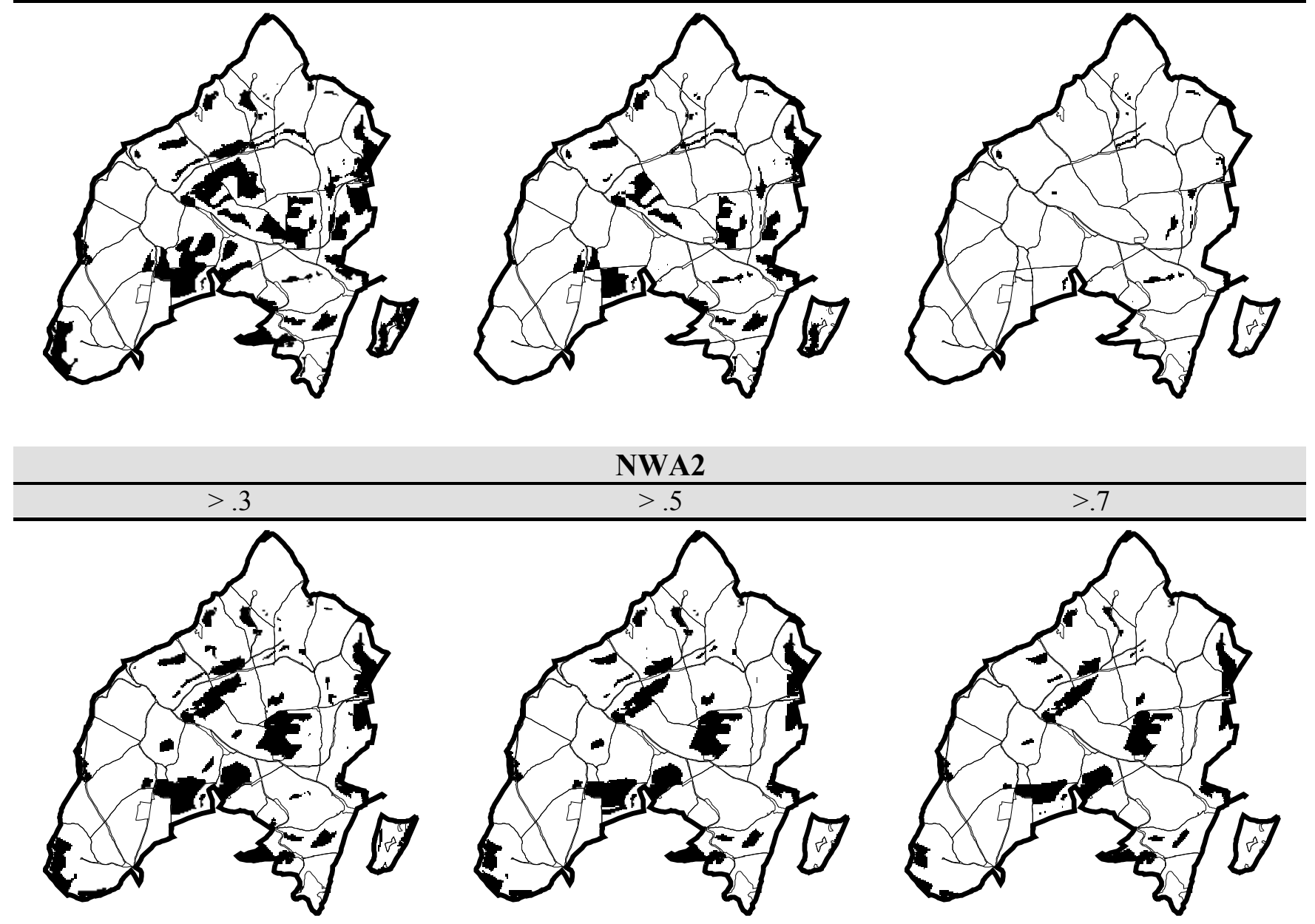

In den Abbildungen 105 und 106 sind die Ergebnisse der in der Tabelle 32 eingeführten Entscheidungsmodelle dargestellt. Jede Abbildung zeigt eine der möglichen Entscheidungen, die sich aus den abgebildeten Präferenzen und dem gewählten Schwellenwert ergeben. 
OWA1 (MAX)
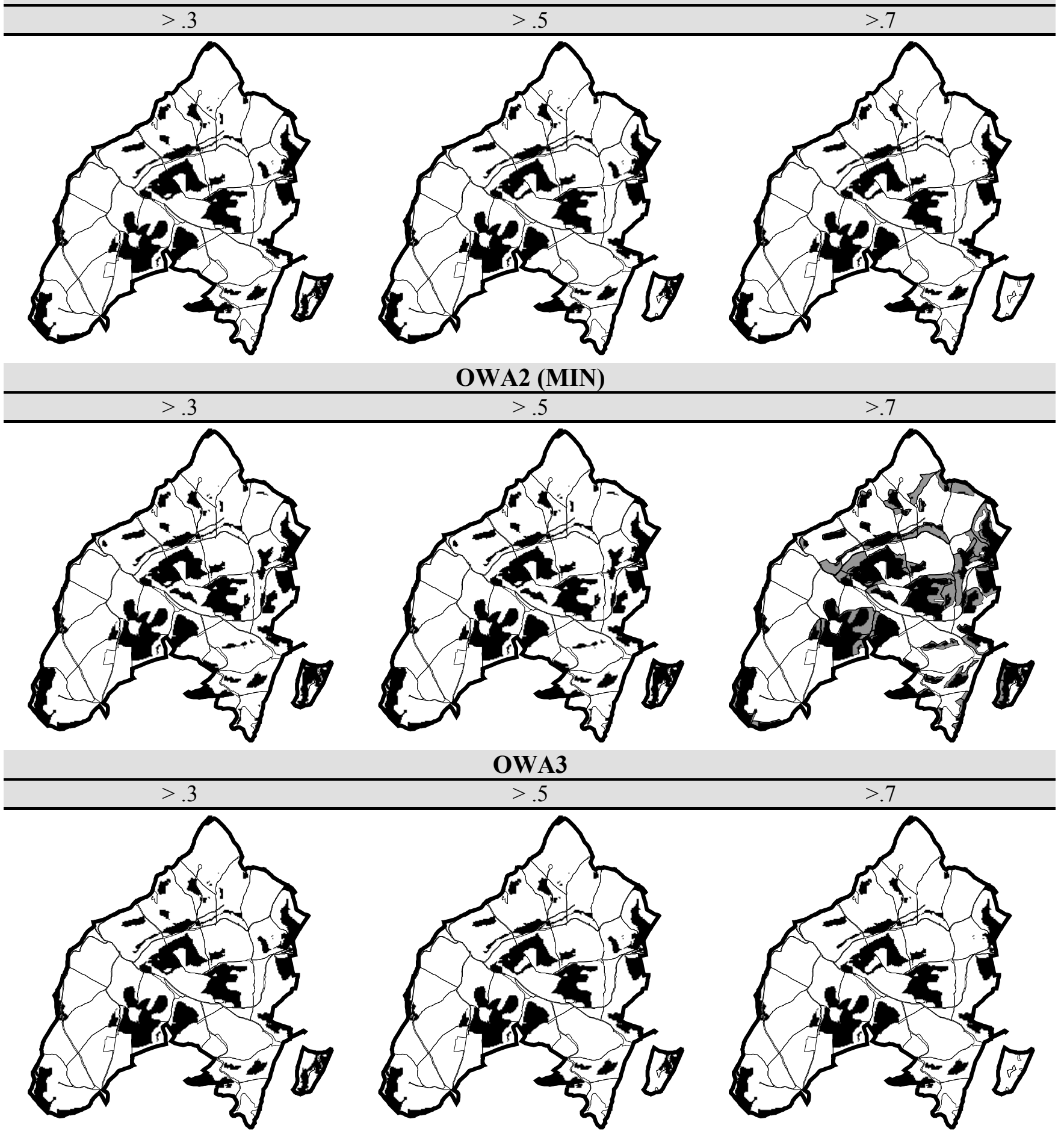

Abb.106: Die Ergebnisse des Ordered Weighted Averaging (Modelle OWA1, OWA2, OWA3 nach der Tabelle 32) mit Schwellenwerten (Mindestwerten) $[.3, .5, .7]$. Alle Einsatzorte, deren Nutzenwert größer ist als der Schwellenwert (Mindestwert), werden für den Harvestereinsatz vorgesehen. Die schwarzen Flächen zeigen die Flächen, wo der Harvestereinsatz nach der modellierten Präferenz als vorteilhaft angesehen wird. 
Die Entscheidungssuche erfolgt in zwei Schritten: Zuerst werden die Entscheidungskriterien (außer der Konzentration) bewertet und zur Gesamtpräferenz verknüpft. Mit Hilfe eines Mindestwertes der Gesamtpräferenz werden Einsatzorte gewählt, die aufgrund ihrer eigenen Eigenschaften als geeignet für den Harvestereinsatz erscheinen. Im zweiten Schritt wird die räumliche Konzentration als letztes Entscheidungskriterium angewendet, unter Berücksichtigung von welchem auch weitere, im ersten Schritt nicht ausgewählte Einsatzorte den Schwellenwert überschreiten können. Abbildung 107 zeigt die Konzentration der Einsatzorte als Dispersion der Präferenzen der im ersten Schritt ausgewählten Einsatzorten: Während (links) ein ,kummulierter' Einfluß als Summe der dispersen Präferenzen von verschiedenen nahe liegenden Orten erlaubt ist, wird (rechts) nur der größte Einfluss (Maximum der sich überschneidenden dispersen Präferenzen) eines nahe liegenden Einsatzortes modelliert.
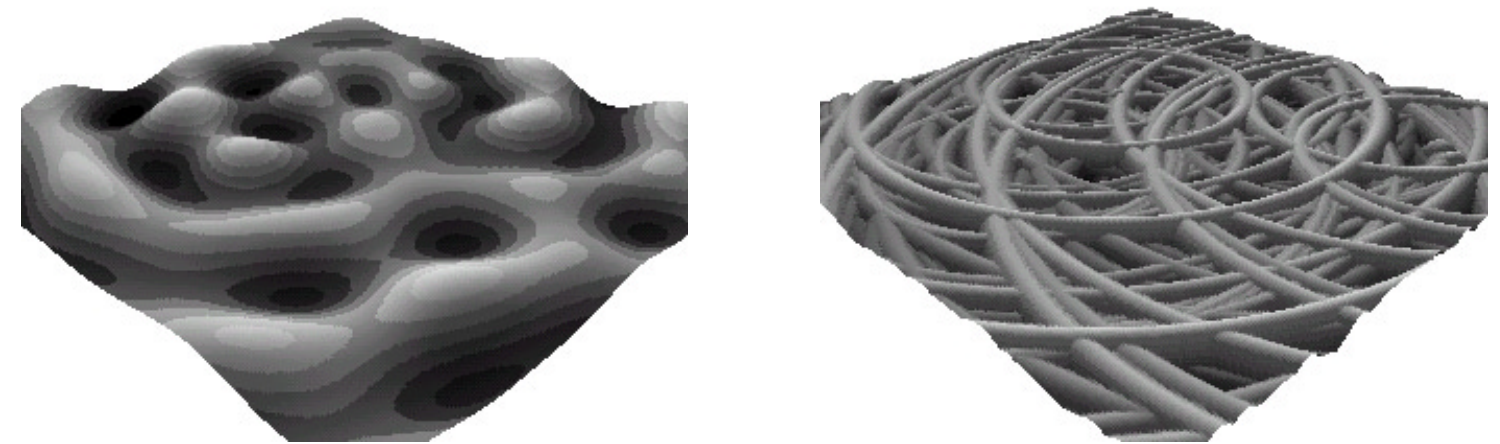

Abb. 107 : Konzepte der räumlichen Konzentration der Einsatzorte als distanzbasierte Dispersion der Präferenzwerte: (links) Summe und (rechts) maximaler Wert der über das Modellgebiet verteilten Präferenzen. Als zugrundeliegende Entscheidung wurde die des Modells NWA mit dem Schwellenwert 0.5 angewendet.

Abbildung 108 zeigt die Auswirkung des Kriteriums ,räumliche Konzentration' im Detail- und Übersichtsansicht. Während die schwarzen Flächen die bereits im ersten Schritt ausgewählten Orte darstellen, zeigen die grauen Flächen die unter Berücksichtigung der räumlichen Konzentration dazugekommenen Einsatzorte.

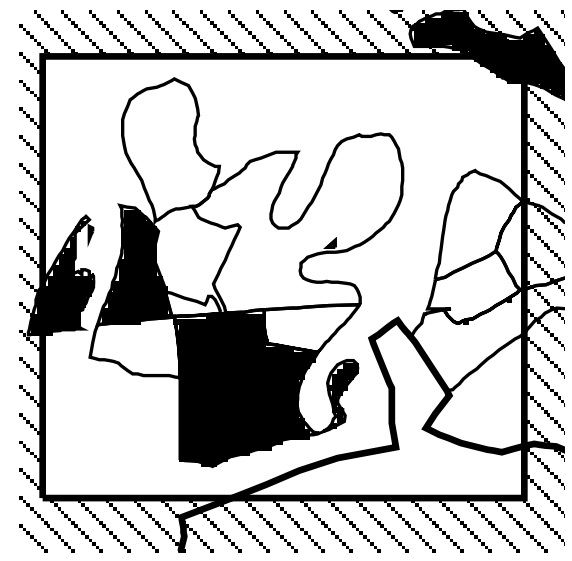

$1: 16000$
M

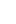

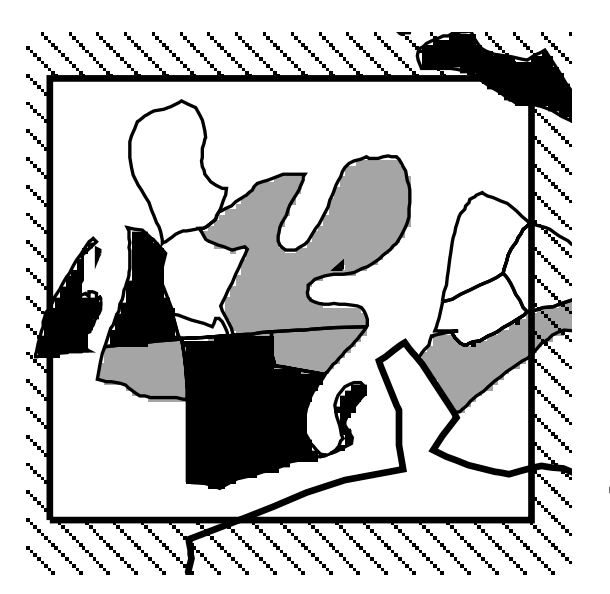

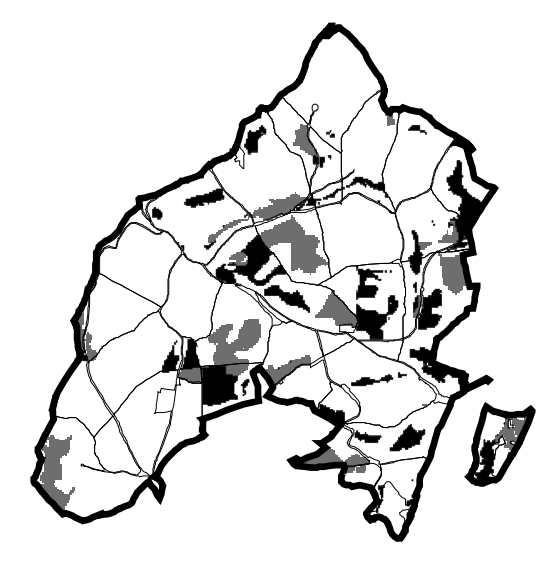

$1: 60000$

Abb. 108: Der Einfluß der Konzentration von Einsatzorten auf die Entscheidung: (links) Detailansicht der Entscheidung von Modell NWA (0.5) ohne und (Mitte) mit Berücksichtigung der Konzentration als Entscheidungskriterium, (rechts) Vergleich der beiden Entscheidungen in der Übersichtsansicht. 
Beide Entscheidungsregeln (NWA und OWA) kontrollieren unterschiedliche Inhaltspunkte der Entscheidungssituation: Während die Nutzwertanalyse eine volle Kompensation einer schlechten Ausprägung eines Kriteriums durch eine bessere in einem anderen erlaubt, richtet sich die OWA auf die Kontrolle dieser Kompensation zwischen den extremen Minimax- und MaximaxEntscheidungen.

Die Ergebnisse der Entscheidungsmodelle unterliegen der Subjektivität der Entscheidungsfindung. Die zu wählende Entscheidungsregel sowie die geäußerten Präferenzen bestimmen die Entscheidung. Die Entscheidungstheorie bietet einen axiomatisch aufgebauten Rahmen des rationalen Verhaltens, ohne dabei die Subjektivität der Zielsetzung und der Präferenzen bezüglich der Konsequenzen einer Entscheidung einzuschränken.

Die hier behandelte Entscheidungssituation zielte mit dem Schwerpunkt auf die Modellierung von entscheidungsrelevanten räumlichen Phänomenen. Die formale Ergebnis- und Sensitivitätsanalyse ist nur unter Einbeziehen konkreter Präferenzen eines Entscheidungsträgers möglich. Die Geoinformationsverarbeitung hilft im Prozeß der räumlichen Entscheidungsfindung dabei, die Präferenzen anhand geeigneter Konzepte abzufragen und räumlich umzusetzen. Die Abbildung der Präferenzen sowie ihre Aggregation ist Gegenstand der Entscheidungstheorie. Welche Entscheidung besser ist, kann die (präskriptive) Entscheidungstheorie anhand der Axiome des rationalen Verhaltens beurteilen. 


\section{Diskussion und Zusammenfassung}

Die vorliegende Arbeit befasst sich mit der Geoinformation und ihrem Beitrag zur Entscheidungsfindung an Beispielen aus der Forstwirtschaft. Sie verfolgt das Ziel, räumliche Entscheidungssituationen bezüglich der Konzepte zur Präferenzabbildung und Strukturierung zu untersuchen und ihre Anwendung in forstlichen Handlungssituationen aufzuzeigen.

Die Motivation der Arbeit liegt in der wachsenden Bedeutung der Geoinformation sowie dem steigenden Bedürfnis nach ihrer effektiven Einbeziehung zur Lösung räumlicher Entscheidungsprobleme. Dieses wird vor allem durch wachsende Zielkonflikte der Raumnutzung und die dadurch bedingte Verflechtung von räumlichen Konzepten verschiedener beteiligter Gruppen verursacht. Die postindustrielle Wissensgesellschaft wird durch größeres Wissen, größere Komplexität und wachsende Turbulenzen charakterisiert. Diese Veränderungen spiegeln sich in der Zunahme von entscheidungsrelevanten Gruppentreffen und ihrer Bedeutung wider. Zur gleichen Zeit werden die Entscheidungen immer komplexer und müssen in immer kürzer werdenden Zeitabständen in immer größer werdenden Gruppen getroffen werden. Die effektive Lösung räumlicher Entscheidungsprobleme setzt daher ein räumliches Modellbewusstsein der Entscheidungsträger voraus, das es erlaubt, die Präferenzen bezüglich der räumlichen Konsequenzen der Alternativen ableiten und aggregieren zu können.

Die Mehrheit der Entscheidungen in der Forstwirtschaft hat einen räumlichen Bezug oder eine räumliche Auswirkung. Die forstliche Planung ist eine raum-zeitliche Planung, da alle Prozesse des Waldes raum-zeitlich gebunden sind. Zur Abbildung der natürlichen, technologischen und ökonomischen Prozesse sind deshalb raum-zeitliche Konzepte und Prinzipien von Bedeutung. Positionale Aspekte sind in der räumlichen Ordnung des Waldes und der Nutzungsplanung enthalten, haben Einfluss auf die Stabilität des Waldes und die Produktionssicherheit, spiegeln sich in den Kosten und Einnahmen der Bewirtschaftung wider. Sind der angestrebte Nutzen oder mit ihm verbundene Fragen räumlich differenziert, d.h. besitzen die Ziele des Entscheidungsträgers räumliche Aspekte, so weisen auch die zugrundeliegenden Entscheidungen einen räumlichen Charakter auf.

Raum ist ein grundsätzliches Strukturierungskonzept (s. Kap. 3.1). Der geographische Raum wird durch subjektive räumliche Beobachtungen mit unterschiedlicher Skalierung und Auflösung wahrgenommen. Er wird aus einem Zentrum (HIER: Position des Betrachters) beobachtet, von welchem aus nur ein Ausschnitt sichtbar ist. Die durch Wahrnehmen, Messen oder Einschätzen und Vermessen eines geographischen Phänomens entstandene Information wird als Geoinformation (GI) bezeichnet. Geoinformationen unterscheiden sich von anderen (aräumlichen) Informationen durch einige Eigenschaften, die auch im weiteren ihre Handhabung und Verarbeitung prägen. Sie sind als Grundlage des planerischen Handelns von großer Bedeutung. Rund $80 \%$ aller Informationen haben räumlichen Bezug. Das Verstehen und Verarbeiten von Geoinformationen ist mit den zugrundeliegenden räumlichen Konzepten untrennbar verbunden.

GIScience (Geoinformationswesen), die Wissenschaft über räumliche Konzepte (s. Kap. 3), wurde vor allem durch die NCGIA (National Center for Geographic Information and Analysis, eine Forschungseinrichtung der National Science Foundation) und UCGIS (University Consortium for Geographic Information Science; ein Zusammenschluß von 34 US Universitäten) 
vorangetrieben. Im Varenius Projekt der NCGIA werden drei Forschungsbereiche der GIScience angenommen, welche alle eine Bedeutung für räumliche Entscheidungsfindung besitzen: (i) Ebene des Individuums als Benutzer der Technologie, als Beobachter des geographischen Phänomens, als Quelle der Konzeptualisierung und als Entscheidungsträger. (ii) Ebene des Systems als gesamte Komplexität der digitalen GI-Technologien und ihrer Unterstützung durch Software, Hardware und Netzwerke. (iii) Ebene der Gesellschaft, einschließlich der Institutionen, Kunden, Gruppen, Normen und Standards mit gegenseitiger Beeinflussung der Technologie und der Gesellschaft.

Aus den Forschungsthemen der GIScience wurden in dieser Arbeit vor allem die Geokognition und die Geodatenmodellierung ausführlicher behandelt, da sie von großer Bedeutung für die räumliche Entscheidungsfindung sind. Die geographische Kognition behandelt Themen, welche für die räumliche Entscheidungsfindung von großer Bedeutung sind: Wie Menschen geographische Information lernen und wie sie zur Entscheidungsfindung angewendet wird, wie die Menschen räumliche Konzepte entwickeln und wie sie anhand dieser handeln, wie komplexe Geoinformation dargestellt werden kann, um Verstand und Entscheidungsfindung zu unterstützen etc. Geographische Datenmodellierung geschieht im Prozess der Abstraktion eines räumlichen Phänomens nach vorgegebenen Regeln, die den Inhalt und die Weise der Repräsentation und Beschreibung festlegen. Die Form der Repräsentation ist für die Akzeptanz sowie für die Effektivität der Benutzung von geographischen Informationstechnologien und daher auch für die Anwendung und Interaktion mit einem räumlichen Entscheidungsunterstützungssystem von großer Bedeutung.

Entscheidungsrelevante Elemente einer räumlichen Situation setzen sich aus räumlichen Objekten, ihren Beziehungen sowie der Dynamik beider zusammen. Ein Geoobjekt ist ein auf einen räumlichen Ausschnitt der Erde bezogenes reales oder gedankliches Objekt. Räumliche Eigenschaften entscheidungsrelevanter Objekte (s. Kap. 5) müssen zielbezogen bewertet werden. Dabei werden vor allem die positionsbezogenen Aspekte (relative Position) wichtig, die anhand räumlicher Beziehungen beschrieben werden können (s. Kap. 5.2). Darüber hinaus können auch individuelle Eigenschaften wie Form, Größe, Ausdehnung und Ausrichtung zur Entscheidungsfindung beitragen (s. Kap. 5.1). Diese sind für die räumliche Entscheidungsfindung nur so weit interessant, inwieweit sie die relative Position der entscheidungsrelevanten Objekte beeinflussen. Für die Entscheidungsfindung können statt der genauen Geometrie eines räumlichen Objektes vereinfachend seine Approximation, Teile seiner Zerlegung oder Abstraktionen herangezogen werden.

Die Theorie räumlicher Beziehungen behandelt die Arten und Eigenschaften der Beziehungen zwischen räumlichen Objekte: Im geographischen Raum sind vor allem topologische Beziehungen, Distanz- und Richtungsbeziehungen von Bedeutung (s. Kap. 5.3). Die Ähnlichkeit und konzeptionelle Nachbarschaft der Beziehung sowie ihre Komposition induzieren Möglichkeiten räumlicher Inferenz. Die Kombination verschiedener Beziehungsarten und ihre Komposition zur Ableitung unbekannter Beziehungen oder zur Verminderung der Unsicherheit nicht vollständig bekannter oder unsicherer Beziehungen (räumliches Schließen) ist Gegenstand einer Relationen Algebra.

Die topologischen Beziehungen stellen primär ein qualitatives Konzept dar (s. Kap. 5.3.2). Sie bezeichnen räumliche Beziehungen, die unter Transformationen wie Translation, Skalierung oder Rotation erhalten bleiben. Sie beschreiben die Konzepte der Nachbarschaft, des Enthaltenseins 
und Überschneidens. Für topologische Beziehungen wurden mehrere Beziehungsmodelle entwickelt. Sie basieren auf Eigenschaften, welche im Prozess topologischer Transformation unverändert (invariant) bleiben. Die Inhaltsinvariante ist eine einfache topologische Invariante, bei welcher der Inhalt von Schnittmengen einzelner Objektteile betrachtet wird. Weitere Invarianten wie beispielsweise die Dimension der Schnittmenge und die Anzahl der Komponenten sind für eine Verfeinerung der topologischen Beziehungen von Bedeutung.

Die Distanz bezeichnet eine Beziehung zwischen einem Primärobjekt und einem Referenzobjekt in einem Bezugssystem (s. Kap. 5.3.3). Sie gibt die relativen Positionen oder GrößenEigenschaften an. Die Bestimmung der Entfernung setzt die Existenz einer Metrik $d$ voraus. Menschliche Entfernungskonzepte hängen dabei von vielen Faktoren ab: Metrische Entfernung, Zeitaufwand, Kosten, Wahrnehmung, relative Größe und Form, Position anderer Objekte, Bezugsrahmen. Die Messgröße der Entfernung muss diese Konzepte in einer konkreten Situation berücksichtigen. Sie kann eine räumliche (metrische Entfernung), zeitliche (Reisezeit), ökonomische (Reisekosten) oder kognitive (räumliche Wahrnehmung) Größe sein.

Die Richtungsbeziehungen beschreiben die Ordnung des Raumes (s. Kap. 5.4). Sie geben an, wo die Objekte relativ zueinander liegen. Eine Richtungsrelation entsteht durch die Projektion antropomorph bestimmter Achsen auf das Bezugsobjekt. Bei der Bestimmung der Beziehung benötigt man ein primäres Objekt, dessen relative Position zu bestimmen ist, ein Referenzobjekt, im bezug auf welches die Richtung bestimmt wird, und einen Bezugsrahmen. Richtungsbeziehungen haben eine uniforme kreisförmige Nachbarschaftsstruktur. Man unterscheidet zwei Ansätze der Richtungsbeziehungen: die projektionsbasierten Relationen und die Relationen der konischen Bereiche (Kegelform-Ansatz) (vgl. Kap. 5.4).

Die Entscheidungstheorie ist eine interdisziplinäre Forschungsrichtung, die sich mit der Formulierung und der Lösung von Entscheidungsproblemen beschäftigt (s. Kap. 6). Die präskriptive Entscheidungstheorie befasst sich primär mit der Rationalität der Entscheidungsfindung. Sie beschreibt nicht die Realität, sondern gibt Verhaltensempfehlungen für alternative Entscheidungssituationen. Sie zeigt, wie Entscheidungen 'rational' getroffen werden können. Die Rationalität stellt bestimmte Anforderungen an die Eigenschaften der Alternativen und an das Verhalten des Entscheidungsträgers.

Die Präferenz ist die Einsicht eines Entscheidungsträgers, aufgrund welcher er eine Alternative einer anderen vorzieht oder beide als gleichwertig ansieht (s. Kap. 6.6). Zur Lösung eines Entscheidungsproblems wird die Präferenz abgebildet und gemessen. Eine zukunftsorientierte Entscheidung zeichnet sich dadurch aus, dass die Präferenz eines ETs aus einer Bewertung der Konsequenzen der Alternativen abzuleiten ist. Auf den Mengen der Entscheidungsalternativen werden durch binäre Relationen Ordnungsstrukturen eingeführt. Die Äquivalenz- und Ordnungsrelationen stellen die Grundlagen der mathematischen Entscheidungstheorie dar. Sie werden auch Indifferenz- und Präferenzrelationen genannt. Eine Präferenzrelation ist eine Relation, die vollständig und transitiv ist.

Eine Präferenz, welche die relative Lage und die räumlichen Konsequenzen der Alternativen zielbezogen anordnen kann, wird als räumliche Präferenz bezeichnet. Vor allem die Theorie räumlicher Beziehungen, ein zentraler Forschungsbereich der GIScience, ist für räumliche Entscheidungsfindung von großer Bedeutung (s. Kap.7). Anhand positionsbezogener Aspekte räumlicher Objekte können Unterschiede der Alternativen erfasst und bewertet werden. Die 
anhand der räumlichen Konzepte abgebildeten Präferenzen werden mit Hilfe von Entscheidungsmodellen verknüpft (s. Kap. 6.7). Dadurch wird eine Anordnung der Alternativen sowie die transparente Darstellungsweise des Entscheidungsprozesses möglich.

Die räumliche Entscheidungsfindung ist ein Bestandteil der Entscheidungstheorie, welche auf die Anwendung von Entscheidungsmethoden und Modellen in der räumlichen, insbesondere der geographischen Domäne ausgerichtet ist (vgl. Kap. 7). Sie behandelt Handlungssituationen, in welchen die Räumlichkeit für die Beurteilung der Alternativen von Bedeutung ist. Sie nutzt räumliche Konzepte zur Abbildung der Präferenz eines Entscheidungsträgers. Die wichtigste Aufgabe räumlicher Entscheidungsfindung ist es, mit Hilfe formaler und implementierbarer Methoden dem Entscheidungsträger die Formulierung seiner Kriterien und die Abbildung seiner Präferenzen über Alternativen zu ermöglichen. Die mentalen Modelle werden dabei in formale überführt, auf Konsistenz überprüft, verarbeitet, um schließlich durch die Präferenzabbildung eine Lösungsfindung zu ermöglichen. Die räumlichen Entscheidungsunterstützungssysteme sind Werkzeuge, welche den Entscheidungsträger durch Generierung oder Bewertung von räumlichen Alternativen oder durch Handhabung von entscheidungsrelevanten räumlichen Objekten und Beziehungen unterstützen.

Räumliche Entscheidungsfindung findet eine Anwendung in allen Bereichen, in welchen zwischen alternativen Handlungen mit unterschiedlichen räumlichen Konsequenzen entschieden werden soll. Fragestellungen wie Ausscheidung potentieller Schutzgebiete, Entscheidungen über Flächennutzungen, flächenbezogene Bewertungen, Bestimmung des Absatzgebietes oder Standortsuche können mit Hilfe räumlicher Entscheidungsfindung gelöst werden (vgl. Kap. 7.1).

Die räumliche Entscheidungsfindung kann in der Forstwirtschaft (s. Kap. 8) eine Anwendung vor allem in der Planung räumlicher Ordnung finden. Ihre primäre Aufgabe ist es, durch Aufbau und geographische Anordnung der Bestände sowie durch Ausnutzung und Schaffung natürlicher und künstlicher Schutzvorrichtungen das Produktionsrisiko gering zu halten und zu verteilen. Sie erstrebt einen dynamisch-strukturellen Waldaufbau, durch den biologische, ökologische, technologische und ökonomische Forderungen zur Ausschöpfung des Ertragsvermögens, zur Minderung des Produktionsrisikos, zur Erleichterung von Holzernte und Verjüngung und zur Erhöhung der landeskulturellen Leistungen in Überstimmung gebracht werden (KURTH et al. 1994). Räumliche Ordnung ist ein komplexes Konglomerat von einander abhängigen Entscheidungen mit unterschiedlicher Bedeutung für die gesamte Ordnung, unterschiedlichen Beeinflussungsmöglichkeiten und entscheidungsrelevanten Beziehungen. Die Entscheidungen, deren Ziel Schaffung einer günstigen räumlichen Ordnung ist, können sich in ihrer räumlichen Skalierung, ihrer Hierarchieebene, dem mit ihnen verbundenen Risiko sowie ihren zeitlichen Auswirkungen unterscheiden. Wegen vielfältiger Interdependenzen ist die Schaffung günstiger räumlicher Ordnung das komplexeste Ziel der Forstwirtschaft. Die Ansätze und Modelle der räumlichen Ordnung sind durch starre Leitbilder gekennzeichnet. Der Grund dafür liegt vor allem im Aufwand einer solchen Entscheidungsfindung, deren effektive Bearbeitung erst in den letzten Jahren ermöglicht wurde.

Die Bewältigung neuartiger Aufgaben stellt erhöhte Anforderungen an die Fähigkeit der Forstleute, räumliche Konzepte zu formulieren und anzuwenden. Bei räumlichen Entscheidungen wie beispielsweise bei Biotopschutz oder Reservatausweisung werden forst-fremde Konzepte zusammen mit forstlichen (Nutzungsart, -dauer) angewendet. Die aktive Teilnahme der Forstwirtschaft an diesen Entscheidungen setzt die Formulierung einer eigenen räumlich 
ausgeprägten Präferenz der Forstleute voraus, die sich auf die relevanten räumlichen Konzepte stützt.

In der Arbeit werden ausgewählte räumliche Handlungssituationen modelliert, in welchen Entscheidungsprobleme auftreten. Die behandelten Beispiele (Entscheidungen über Technologieeinsatz, Wahl der Waldentwicklungszieltypen, Beurteilung der Alternativen bei der Walderschließung und Modellierung der räumlichen Situation bei der Verjüngungsplanung) stellen nur eine kleine Auswahl dar (s. Kap. 8.3). Die Beispiele beschreiben schematisch die Elemente räumlicher Szenen sowie die Alternativen- und Kriterienbildung. Das Ziel dieser Beispiele ist es, die Formulierung und Anwendung von räumlichen Konzepten zu zeigen, die zur Kriterienbildung und Alternativenbeurteilung herangezogen werden können. Die Beispiele der Handlungssituationen sind so formuliert, dass sie die Anwendung der räumlichen Entscheidungsfindung sowie die Struktur räumlicher Entscheidungsprobleme näher erläutern. Sie stärken das Problembewusstsein für räumliche Entscheidungsfindung und ihre Einsatzmöglichkeiten in der Forstwirtschaft.

Die Beispiele werden auf die Räumlichkeit und ihren Einfluss auf die Entscheidungsfindung beschränkt. Die thematischen Eigenschaften der Geoobjekte werden nur dann explizit herangezogen, wenn es für die räumliche Komponente der forstlichen Geoobjekte nötig wird. In Beispielen werden Konzepte vorgestellt. Eine Lösung von konkreten Entscheidungsproblemen, für welche Geodaten eingesetzt werden können, setzt die Äußerung von Präferenzen eines Entscheidungsträgers voraus. Die gefundene Lösung stellt dann jedoch nur die 'optimale' Lösung für die gegebene Zielsetzung und Präferenzen dar. Eine Verallgemeinerung solcher Lösungen ist nicht möglich. Aus diesem Grund wurde bei der Formulierung der Beispiele den räumlichen Konzepten größere Bedeutung beigemessen. Im Kapitel 9 wird anschließend der Entscheidungsprozeß an einem konkreten Entscheidungsproblem anhand der Geoinformationsbasis des Betriebsbezirkes Lichtenhagen (Forstamt Reinhausen) vorgestellt. Die Fragestellung des Beispieles stammt aus SPORS et al. (1992). Im Rahmen dieser Arbeit wurde die Fragestellung erweitert und in ein räumliches Entscheidungsproblem überführt, dessen Lösung modelliert wird.

Die Fähigkeit, räumliche Konzepte $\mathrm{zu}$ entwickeln und in Prozessen der mentalen Informationsverarbeitung anzuwenden, ist eine primäre Kognitionsfähigkeit der Menschen (vgl. Kap. 3.4 und 7.1). Die mentalen räumlichen Modelle (kognitive Karten) sind für die Planung komplexer Sachverhalte nicht zuverlässig. Sie enthalten nur bestimmte Aspekte der Räumlichkeit und nur vage Informationen. Der Mensch hat bereits zu Beginn seiner geographischen Erkundung einfache Abbildungen der Räumlichkeit in Form von Karten benutzt, um diese Unzulänglichkeiten seiner kognitiven Modelle zu überwinden. Im komplexen System mehrerer Ziele im Umbau des forstlichen Paradigmas scheint die computergestützte Planung heute ein unausweichliches Mittel zur Erreichung der angestrebten Ziele zu sein.

Die räumliche Entscheidungsfindung stellt ein Mittel dar, das für die Forstliche Planung in der geänderten Situation der Waldbewirtschaftung von großer Bedeutung sein wird. In den zunehmenden Konflikten der Raumnutzung ist rationale und transparente Entscheidungsfindung gefragt. Um ihre Möglichkeiten jedoch nutzen zu können, muss die Räumlichkeit forstlicher Planung genauer untersucht werden: (i) Konzepte verträglicher Nutzung müssen räumlich in Struktur und Beziehungen aufgelöst und ihr Präferenzrahmen muss aufgezeigt werden. (ii) Die Präferenzunabhängigkeit bezüglich räumlicher Objekte und ihrer Beziehungen in verschiedenen 
Teilbereichen der Forstwirtschaft muss getestet werden. (iii) Die Interdependenzen zwischen den forstlichen Entscheidungen mit räumlichen Auswirkungen müssen untersucht werden.

Abschließend bleibt festzuhalten, dass räumliche Entscheidungsfindung, GIScience und räumliche Entscheidungsunterstützungssysteme (SDSS) Methoden und Werkzeuge zur Verfügung stellen, deren Anwendung in der Forstwirtschaft im Prozess der Entscheidungsfindung unverzichtbar ist. Ihr Potential liegt vor allem in der Handhabung von raum-zeitlichen Beziehungen, deren Verständnis und Beeinflussung die Regelung des Waldes prägen. Sie ergänzen das forstliche Planungsmodell um die Fähigkeit, räumlich und zeitlich begründete Entscheidungsvarianten bei den forstlichen Entscheidungen heranzuziehen und deren Präferenzen abzufragen. Mit zunehmender Komplexität von Raumnutzungskonflikten steigt die Notwendigkeit, die Entscheidungsträger in der Forstwirtschaft mit den Möglichkeiten der räumlichen Entscheidungsfindung vertraut zu machen. 


\section{Literaturverzeichnis}

Aalders H.J.G.L. (1996): Quality metrics for GIS. In: Kraak M.-J., Molenaar M. (eds.): Seventh International Symposium on Spatial Data Handling (SDH '96), Taylor\&Francis, S.277-286.

Albrecht J. (1995): Universal Analytical GIS Operations. Dissertation an der Universität Vechta.

Allen J. (1983): Maintaining Knowledge about temporal intervals. Communications of the ACM, 26 (11), S.832843.

Altman D. (1994): Fuzzy set theoretic approaches for handling imprecision in spatial analysis. International Journal of Geographical Information Systems 8, S.271-289

Anonymus (1994): Executive office of the president: Coordinating geographic data acquisition and access: the National Spatial Data Infrastructure Executive Order 12906. Federal register 59, 17671-4

Anonymus (1996): Research priorities for geographic information science. University Consortium for Geographic Information Science, Cartography and Geographic Information Systems, Volume 23, Number 3 American Congress on Surveying and Mapping. (http://www.ncgia.ucsb.edu/other/ucgis/CAGIS.html)

Anonymus (1998A): Bericht zur Verbesserung der Koordinierung auf dem Gebiet des Geoinformationswesens. Bundesministerium des Inneren. (http://www.ifag.de/IMAGI/Bericht.htm)

Anonymus (1998B): Geographic Information in Europa: a discussion document. GI 2000 Programm der Europäischer Kommision. (http://158.169.50.95:10080/gi/en/gi2000/discussion98.html)

Anonymus (1998C): Public participation in decision-making about forests: a statement by the canadian institute of forestry. Institut forestier du Canada. (www.cif-ifc.org/publicparticipatione.html)

Anonymus (1999A): National centre for geographic Information Analysis NCGIA. Home Page (http://www.ncgia.ucsb.edu)

Anonymus (1999A1): OpenGIS® Abstract Specification: Feature Geometry Topic 1. OpenGIS Consortium. (http://www.opengis.org)

Anonymus (1999A2): OpenGIS® Abstract Specification: The OpenGIS Feature Topic 5. OpenGIS Consortium. (http://www.opengis.org)

Anonymus (1999B): Wege zur Wissenschaftstheorie. Vorlesungmaterialien im Fachbereich Erziehungswissenschaften und Biologie Universität Dortmund. (http://www.fb12.unidortmund.de/wtheorie/JPEG)

Anonymus (1999C): Conference on Spatial Information Theory (COSIT). Home Page. (http://www.informatik.unihamburg.de/WSV/cosit99)

Anonymus (1999D): FAQ concerning geographic information. Direction Generale XIII/E der Europäischen Kommision (http://158.169.50.95:10080/gi/en/gi2000/gi-faqs.html)

Anonymus (1999E): Association of Geographic Information Laboratories in Europe (AGILE), Home Page. (http://www.uniroma1.it/DICEA/AGILE.htm)

Anonymus (1999F): Forum Threads: Geospatial decision support system. Federal Geographic Data Committee (http://www.fgdc.gov/99Forum/threads.html)

Anonymus (1999G): Collaborative GIS and SDSS Global Environmental Change. Data Assessment and Integration Project Home Page. (http://www.ciesin.colostate.edu/USDA/Task\%203\%20Web/97T31.html)

Anonymus (2000A): Enzyklopädie 2000. In: Meyer A., Seibert G., WendelbergerE. (eds). Wissen Verlag. 1969 Stuttgart/ Zürich

Anonymus (2000B): University Consortium for Geographic Information Science (UCGIS). Home page. (http://www.ucgis.org) 
Anonymus (2000c): GIS Interoperability Project Stimulating the Industry in Europe (GISPIE). Home page. (http://ifgi.uni-muenster.de/3_projekte/forschungsberichte/gipsie).

Anonymus (2000D): Mitteillung der Kommission. Europäische Kommision. (http://158.169.50.95:10080/info2000/de/mitteilung.html)

Anselin L. (1989): What is special about spatial data? Alternative perspectives on spatial data analysis. Technical report 89-4 National centre of Geographic Information Analysis (NCGIA).

Anselin L. (1995): Local indicators of spatial association-LISA. Geographical analysis 27 S.93-115.

Anselin L., Bao S. (1997): Exploratory spatial data analysis linking SpaceStat and ArcView. In Fisher P., Getis A. (eds): Recent Advances in spatial analysis: Spatial statistics, behavioural modelling and computational intelligence, Springer Verlag S.35-59.

Aplin G. (1983): Order-neighbour analysis. Concepts and techniques in modern geography. CATMOG 36, Institut of British Geographers.

Arentze T.A, Borgers A.W.J., Timmerman H.J.P. (1996): Integrating GIS into the planning process. In: Masser I., Salge F.: Spatial Analytical Perspectives on GIS, GISDATA 4, Taylor\&Francis

Artale A., Franconi E., Guarino N., Pazzi L. (1996): Part-Whole Relations in Object-Centered Systems: An Overview. Data\&Knowledge Engineering

Bachmann et al. (1996): Neue Wege der forstlichen Planung. Umwelt-Materialien 45, Wald. Bundesamt für Umwelt, Wald und Landschaften (BUWAL), Bern.

Baczkowski A.J., Joanes D.A., Shamia G.M (1997): Properties of a generalized diversity index. J.theor.Biol. 188 S.207-213.

Bartelme A. (1995): Geoinformatik: Modelle, Strukturen, Funktionen. Springer Verlag Berlin

Batty M., Densham P. J. (1996): Decision Support, GIS, And Urban Planning. Systemma Terra, V(1) S.72-76 (http://www.geog.ucl.ac.uk/ pdensham/s_t_paper.html)

Behr F.J (1997A): Datenanalyse und -präsentation in Geoinformationssystemen. Graphservice White Papers. (http://graphservice.de/papers/analyse.htm)

Behr F.J (1997B): Auskunft - der zentrale Dienst eines Geo-Informationssystems. Graphservice White Papers. (http://graphservice.de/papers/auskunft.htm)

Biethahn J., Mucksch H., Ruf W. (1990): Ganzheitliches Informationsmanagement. Oldenbourg Verlag München Wien.

Biethahn J., Mucksch H., Ruf W. (1992): Ganzheitliches Informationsmanagement, Band I:Grundlagen. Oldenbourg Verlag München Wien.

Bill R., Fritsch D. (1991): Grundlagen der Geo-Informationssysteme. Bd. 1, Herbert Wichmann Verlag.

Bill R., Fritsch D. (1996): Grundlagen der Geo-Informationssysteme, Bd. 2 Analysen Anwendungen und neue Entwicklungen. Herbert Wichmann Verlag.

Bitter A.W. (1990): EDV-gestützte Unternehmensführung im Forstbetrieb mit Hilfe eines flächenbezogenen Betriebsinformationssystems. Dissertation Universität Göttingen.

Bitter A.W. (1998): Nachhaltgerechte Inventur und Planung im multifunktionalen Forstbetrieb. Forstwissenschaftliches Centralblatt S.224-230.

Bitter A.W. (1998B): Neue Wege in der Forstplanung. ArcAktuell 1, S.18.

Bittner A. (1996): A Qualitative Model of Geographic Space. In: Kraak, M.J., \& Molenaar, M. (eds.): Proceedings of Int. Symposium on Spatial Data Handling SDH'96, Vol. 2. International Geographical Union IGU.

Bittner T., Frank, A.U. (1998): On Representing Geometries of Geographic Space. In: Poiker, T.K., \& Chrisman, N. (eds.): Proceedings of 8th Int. Symposium on Spatial Data Handling, SDH'98, in Vancouver, Canada, Published by Interna. S.111-122

Bitz, M. (1981): Entscheidungstheorie. Vahlen Verlag. 
Blaschke T. (1997): Map Algebra und Fuzzy Logic in Behörden? Potential und Akzeptanz von GIS-Analysen bei Einbeziehung von räumlicher Unschärfe. Geographical Information Systems GIS 6, S.3-12.

Böckemann D. (1993): Zur Bedeutung der Raumplanung in der modernen Gesellschaft. In: Böckemann D. (ed. i.A. der Fakultät): Studienrichtung Raumplanung, TU Wien, S.4-7.

Böckmann Th.; Saborowski J; Dahm St; Nagel J; Spellmann H. (1998A): Die Weiterentwicklung der Betriebsinventur in Niedersachsen. Forst und Holz 8, S.219 - 226.

Böckmann Th.; Spellmann H.; Hüsig F. (1998B): Neukonzeption und Weiterentwicklung der Forsteinrichtung in Niedersachsen. Forst und Holz 10, S.298-302.

Bogner D. (1999): Entscheidungsgrundlagen in der Regionalentwicklung durch Ökosystemmodellierung. In: Schrenk M. (ed): Computergestützte Raumplanung CORP '99. Selbstverlag des Instituts für EDV-gestützte Methoden in Architektur und Raumplanung der TU Wien. (http://osiris.iemar.tuwien.ac.at/ corp/corp99/ Programm/programm.html)

Bohne E.; Klix W.D. (1995): Geometrie: Grundlagen fuer Anwendungen. Leipzig Fachbuchverlag.

Bothe H.H. (1995): Fuzzy Logic, Einführung in Theorie und Anwendungen. Springer Verlag, zweite erweiterte Auflage.

Bretzke W. R. (1980): Der Problembezug von Entscheidungsmodellen. Tuebingen : Mohr.

Breunig M. (1995): Integration raeumlicher Informationen fuer Geo-Informationssysteme. Dissertation an der Universität Bonn.

Brinkhoff T. (1994): Der spatial join in Geo-Datenbanksystemen. Dissertation, Berichte aus der Informatik Universität München.

Bruns H., Egenhofer M. (1996): Similarity of Spatial Scenes. In: Kraak M.-J., Molenaar M. (eds.): Seventh International Symposium on Spatial Data Handling (SDH '96), Taylor\&Francis S.31-42.

Burrough P. A (1992): Development of intelligent geographical information systems. International Journal of Geographic Information Systems 6/1992, S.1-11.

Burrough P. A., Frank A. U. (1995): Concepts and paradigms in spatial information: are current geographic information systems truly generic? International Journal of Geographical Information Systems, 9 (2), S.101-116.

Burrough P.A. (1996): Natural objects with indeterminate boundaries. In: Burrough P.A., Frank A.U. (eds): Geographic objects with indeterminate boundaries GISDATA II [ESF scientific programme on geographic informations systems; data integration and database design] S.3-28.

Burrough P. A (1997): Environmental modelling with geographical information systems. In: Kemp Z. (ed): Innovations in GIS 4. Papers from the fourth national conference on GIS research UK (GISRUK), Taylor \&Francis, S.143-153.

Burrough P.A., McDonnell R. (1998): Principles of geographical information systems. Oxford University Press.

Buxmann P. (1999): Entscheidungsunterstützungssysteme. Vorlesungsmaterialien SS 1999, Institut für Wirtschaftsinformatik Goethe-Universität Frankfurt am Main.

Cameron M.A., Abel D.J. (1996): A problem model for spatial decision support systems. In: Kraak M.-J., Molenaar M. (eds.): Seventh International Symposium on Spatial Data Handling (SDH '96), Taylor\&Francis, S.89-99.

Cameselle M. (1998): Decision Support Systems. Lehrmaterialien Institut für Informatik Universität Fribourg (http://www-iiuf.unifr.ch/study/courses98-99/DecisionSupport.desc).

Car A., Frank A. (1994): General Principles of Hierarchical Spatial Reasoning - The case of wayfinding. In: Waugh T.C., Healey, R.G. (eds.): Proceedings of Sixth Int. Symposium on Spatial Data Handling, SDH '94, S.646-664

Car A. (1998): Hierarchical Spatial reasoning: a GeoComputational method. In: Proceedings of the 3rd International Conference on GeoComputation, University of Bristol United Kingdom 17 - 19 September 1998 (http://www.geog.port.ac.uk/geocomp/geo98) 
Carver S. (1996A): Collaborative spatial decision making on the Internet. (http://www.geog.leeds.ac.uk/staff/s.carver/hpcsdm.htm\#contents).

Carver S., Openshaw S. (1996B): Using GIS to explore the technical and social aspects of site selection for radioactive waste disposal facilities Working paper MIS School of Geography, University of Leeds S.96-18 .

Carver S. (1999): Developing web-based GIS/MCE: Improving access to data and spatial decision support tools. In Thill J.C. (ed): Spatial Multicriteria Decision Making and Analysis: a geographic information sciences approach; Ashgate Verlag, S.49-76

Caseti R., Smith B., Varzi A.C. (1998): Ontological Tools for geographic representation. In Nicola Guarino (ed.): Formal Ontology in Information Systems Amsterdam, Oxford, Tokyo, Washington, DC: IOS Press (Frontiers in Artificial Intelligence and Applications), S.77-85.

Chrisman A.R. (1997): Exploring Geographic Information Systems. John Wiley \& Sons, Inc. New York.

Church R.L. (1999): Location modeling and GIS. In: Longley P.A., Goodchild M.F., Maguire D.J. (eds): Geographical Information Systems: Principles, Techniques, Applications and Management. Wiley New York, S.293-303.

Claramunt C., Thériault, M. (1995): Managing time in GIS: An event-oriented approach. In: Clifford J., Tuzhilin A. (eds.): Recent Advances on Temporal Databases, Springer-Verlag, Zurich, S.23-42

Claramunt C., Thériault M. (1996): Toward semantics for modelling spatio-temporal processes within GIS. In: Kraak M-J., Molenaar M. (eds.): 7.International Symposium on Spatial Data Handling, Taylor and Francis, Delft, S.47-63.

Claramunt C., Parent C., Thériault M. (1997): Design patterns for spatio-temporal processes. In: Spaccapietra S., Maryanski F. (eds.): Searching for Semantics: Data Mining, Reverse Engineering, Chapman \& Hall, S.415-428

Clementini E., Di Felice P., van Oosterom P. (1993): A Small Set of Formal Topological Relationships for EndUser Interaction. In: Abel D., Ooi B. C. (eds.): Advances in Spatial Databases - Third International Symposium, SSD'93. Lecture Notes in Computer Science LNCS 692, Springer-Verlag, Singapore S.277-295.

Clementini E., Sharma J., Egenhofer M. (1994): Modeling Topological Spatial Relations: Strategies for Query. Processing Computers and Graphics 18 (6), S.815-822.

Clementini E., Di Felice P.,Califano G. (1995A): Composite Regions in Topological Queries. Information Systems 20(7), S.579-594.

Clementini E., Di Felice P., Hernández D. (1995B): Qualitative representation of positional information. Technical Report FKI-208-95, Technical University of Munich.

Clementini E.; Di Felice P. (1996): An algebraic model for spatial objects with undetermined boundaries. In: Burrough P.A., Frank A.U.: Geographic objects with indeterminate boundaries GISDATA II [ESF scientific programme on geographic informations systems; data integration and analysis]. Taylor \& Francis, S.155-170.

Clementini E.; Di Felice P. (1997A): A global framework for qualitative shape description. GeoInformatica 1(1), S.11-27.

Clementini E.; Di Felice P. (1997B): Approximate Topological Relations. International Journal of Approximate Reasoning, S.173-204.

Compton B. (1991): An Implementation of Raster-based Spatial Analysis as a Programming Language. In: Proceedings EGIS '91, S.207-215.

Couclelis H. (1996): Towards an operational typology of geographic entities with ill-defined boundaries. In: Burrough P.A., Frank A.U.: Geographic objects with indeterminate boundaries GISDATA II [ESF scientific programme on geographic informations systems; data integration and analysis] Taylor \& Francis, S.45-56.

Cui Z ,Cohn A G, Randell D A (1993): Qualitative and Topological Relationships in Spatial Databases. In: Abel D., Ooi B. C. (eds): Lecture Notes in Computer Science No. 692, Advances in Spatial Databases, Springer Verlag, Berlin S.296-315.

Czeranka M. (1996): Spatial Decision Support Systems in Naturschutz und Landschaftspflege? Umsetzungsaspekte für die raumbezogene Planung. In: Dollinger F., Strobl J.: Angewandte Geographische Informationsverarbeitung 
VIII = Salzburger Geographische Materialien, Heft 24. Selbstverlag des Instituts für Geographie (http://www.sbg.ac.at/geo/agit/papers96/czeranka.htm)

Czeranka M. (1997): GIS-basierte Entscheidungsunterstützung in der naturschutzorientierten Raumplanung : dargestellt am Beispiel der Eingriffsregelung. Vechta: (SUWV Studien UmweltWissenschaften Vechta) Band 1.

Czeranka M. (1998): Spatial Data Descision Sytems SDSS. GIS Tutorial 3.0 (http://131.220.125.175/theorie/ grundlag/sdss/kap7.htm)

Daniel L. (1992): SDSS for Location Planning, or The Seat of the Pants is Out. GeoInfo Systems, Dez. 1992 (http://www.utexas.edu/depts/grg/gcraft/notes/gisapps/sdss.html)

Densham, P.J (1991): Spatial Decision Support Systems. In: Maguire, D.J., Goodchild M.F., Rhind D.W. (eds.): Geographical Information Systems: Principles and Applications, vol.1, London: Langman, S.403-412.

Densham P.J, Goodchild M.F. (1994): Research initiative 6: Spatial decision support system. Closing report, National Centre of Geographic Information Analysis NCGIA.

Dietz P., Knigge W., Löffler H. (1984): Walderschließung: ein Lehrbuch für Studium und Praxis unter besonderer Berücksichtigung des Waldwegebaus. Parey Verlag.

Dijkmeijer J., de Hoop S. (1996): Topological relations between fuzzy area objects. In: Kraak M.-J., Molenaar M. (eds.): Seventh International Symposium on Spatial Data Handling (SDH '96), Taylor\&Francis, S.377-394.

Dockner E. J., Vetschera R., Gaunersdorfer A. (1999): Betriebswirtschaftliche Entscheidungen. Lehrmaterialien, Institut für Betriebswirtschaftslehre Universität Wien.

Doukas K. (1997): Umweltverträglichkeitprüfung bei der Walderschließung durch den Einsatz moderner Informationstechnologie. Forst und Holz 52, S.250-255.

Duden (1989): Duden : Das große Woerterbuch der deutschen Sprache, Dudenverlag Mannheim.

Eastman J.R. (1999): Multi-criteria evaluation and GIS. In: Longley P.A., Goodchild M.F., Maguire D.J. (eds): Geographical Information Systems: Principles, Techniques, Applications and Management, 2 Auflage, Wiley New York, S.493-501.

Egenhofer M.; Herring J. (1990): Categorizing Binary Topological Relations Between Regions, Lines, and Points in Geographic Databases. Technical Report, Department of Surveying Engineering, University of Maine.

Egenhofer M., Franzosa R. (1991): Point-Set Topological Spatial Relations. International Journal of Geographical Information Systems 5 (2), S.161-174.

Egenhofer M. (1992A): Why not SQL? International Journal of Geographical Information Systems 6 (2), S.71-85.

Egenhofer M. Al-Taha K. (1992B): Reasoning about Gradual Changes of Topological Relationships. In: Frank A., Campari I., Formentini U. (eds.): Lecture Notes in Computer Science, Springer-Verlag,Vol. 639, Theory and Methods of Spatio-Temporal Reasoning in Geographic Space, S.196-219.

Egenhofer M. (1993A): A Model for Detailed Binary Topological Relationships. Geomatica 47 (3\&4), S.261-273.

Egenhofer M., Sharma J. (1993B): Assessing the Consistency of Complete and Incomplete Topological Information. Geographical Systems 1 (1), S.47-68.

Egenhofer M., Sharma J. (1993C): Topological Relations Between Regions in R2 and Z2. In: Abel D. Ooi B.C. (eds.): Lecture Notes in Computer Science, Vol. 692, Advances in Spatial Databases, Springer-Verlag, S.316336.

Egenhofer M. (1994A): Pre-Processing Queries with Spatial Constraints. Photogrammetric Engineering \& Remote Sensing 60 (6), S.783-790.

Egenhofer M. (1994B): Deriving the Composition of Binary Topological Relations. Journal of Visual Languages and Computing 5 (2), S.133-149.

Egenhofer M. , Clementini E., di Felice P. (1994C): Topological Relations between Regions with Holes. International Journal of Geographical Information Systems 8 (2), S.129-144.

Egenhofer M. Franzosa R. (1994D): On the Equivalence of Topological Relations. International Journal of Geographical Information Systems 9 (2), S.133-152. 
Egenhofer M. J., Mark D. (1995B): Modeling conceptual neighborhoods of topological line-region relations. International Journal of Geographical Information Systems 9(5), S.555-565.

Egenhofer M., Mark D. (1995C): Naive Geography. In: Frank A. Kuhn W. (eds.): Conference on spatial information theory (COSIT '95), Lecture Notes in Computer Science, Vol. 988, Springer-Verlag, S.11.- 15.

Egenhofer M. (1996): Geographic Database Systems: Issues and Research Needs. Tutorial Fifteenth ACM SIGACT-SIGMOD-SIGART Symposium on Principles of Database Systems (PODS) Montreal, Quebec, Canada June 4, 1996. (http://www.spatial.maine.edu/ max/pods.html)

Egenhofer M., Mark D.M. (1997): Naive Geography. NCGIA Initiative 21: Formal Models of Common Sense Geographic Worlds; NCGIA Technical Report 97-2 (http://www.geog.buffalo.edu/ncgia/i21/ng/NG51.html)

Egenhofer M. Shariff A. R. (1998): Metric Details for Natural-Language Spatial Relations. ACM Transactions on Information Systems, 16 (4), S.295-321.

Egenhofer M.J., Glasgow J., Günther O., Herring J.R., Peuquet D.J. (1999): Progress in computational methods for representing geographical concepts. International Journal of Geographical Information Science 8/13, S.775796.

Egenhofer M. (2000): Ontologies in GIS. UCGIS Annual Assembly and Summer Retreat Meeting Agendas (http://www.ucgis.org/oregon/agendas.html\#resplen)

Eisenfuehr F., Weber M. (1993): Rationales Entscheiden. Springer Verlag.

Eisenfuehr F., Weber M. (1999): Rationales Entscheiden. Springer Verlag, zweite Auflage.

Enache M. (1994): Integrating Gis With Dss: A Research Agenda. URISA proceedings papers from the Annual conference of the Urban and Regional Information Systems Association, S.154-166 (http:// wwwsgi.ursus.maine.edu/gisweb/spatdb/urisa/ur94015.html)

Enzinger Ch. (1997): Softwareergonomische Evaluation und Redesign am Beispiel des Bibliotheksverwaltungsprogramms BIBOS: Eine Benutzungsfreundlichkeitsanalyse. Diplomarbeit Naturwissenschaftliche Fakultät der Universität Salzburg. (http://www.cosy.sbg.ac.at/ leo/diplomarbeit/ mentalemodelle.html)

Erni V., Lemm R. (1998): Höhere Wertschöpfung durch gezieltes Informationsmanagement. Forum für Wissen, S.61-69.

Erwig M.; Schneider M. (1997): Vague Regions. In: Scholl M. (ed.): Advances in Spatial Databases (SSD) 5th Int. Symp., LNCS 1262, Lecture Notes in Computer Science, Springer Verlag, S.298-320.

Eschenbach C., Habel Ch., Heydrich (1999): Einstellungen, Begriffe und Objekte. Schwerpunkt des Graduiertenkollegs Kognitionswissenschaft Universität Hamburg.

Eastman J.R., Jiang H. (1995): Fuzzy measures in multi-criteria evaluation. In: Proceedings, Second International Symposium on Spatial Accuracy Assessment in Natural Resources and Environmental Studies. May 21-23 (Fort Collins), S. 527-534.

Eastman J.R., Kyem P.A.K., Toledano J., Jin W. (1993): GIS and decision making. Explorations in Geographic Information System Technology. 4 (Geneva: Unitar).

Faißt G., Mayer F.J., Nüßlein S., Zahner V. (1998): GIS-Einsatz an der Bayerischen Landesanstalt für Wald und Forstwirtschaft. ArcAktuell 1, S.14-15.

Falcidieno B., Pienovi C., Spagnuolo M. (1996): Descriptive modelling and declarative modelling for spatial data. In: Masser I., Salge F. (eds): Spatial Analytical Perspectives on GIS, GISDATA 4, Taylor\&Francis, S.139-145.

Fedra K. (1994): Gis and environmental modelling. In: Goodchild M.F, Parks B.O., Steyaert L.T (eds): Environmental modeling with GIS; Oxford university press, S.35-50.

Findeisen D. (1990): Datenstruktur und Abfragesprache fuer raumbezogene Informationen. Schriftenreihe des Instituts fuer Kartographie und Topographie der Rheinischen Friedrich-Wilhelms-Universitaet Bonn; 19, Kirschbaum-Verlag, Dissertation.

Fischer M. M, Nijkamp P. (1991): Geographic information systems and spatial analysis. WSG- discussion Paper 14, Institut für Wirtschafts- und Sozialgeographie Wirtschaftsuniversität Wien. 
Fischer M. M, Nijkamp P. (1992): Geographic information systems and spatial modelling. WSG- discussion Paper 19, Institut für Wirtschafts- und Sozialgeographie Wirtschaftsuniversität Wien.

Fischer M. M., Scholten H.J., Unwin D. (1996A): Geographic information systems, spatial data analysis and spatial modelling: an introduction. In: Masser I., Salge F. (eds): Spatial Analytical Perspectives on GIS, GISDATA 4, Taylor\&Francis.

Fisher P. (1996): Boolean and fuzzy regions. In: Burrough P.A., Frank A.U. (eds): Geographic objects with indeterminate boundaries GISDATA II [ESF scientific programme on geographic informations systems; data integration and database design] Taylor \& Francis, S.87-94.

Fonseca F., Egenhofer M. (1999): Ontology-Based Geographic Information Systems. In: Medeiros C. B. (ed.): 7th ACM Symposium on Advances in Geographic Information Systems, Kansas City, MO, S.14-19.

Fonseca F., Egenhofer M. , Davis C. , Borges K. (2000): Ontologies and Knowledge Sharing in Urban GIS. Computer, Environment and Urban Systems 24 (3), S.251-272.

Forer, P., Unwin D.J. (1997): Enabling progress in GIS and education. In: Longley P.A., Goodchild M.F., Maguire D.J., Rhind D.W. (eds): Geographical Information Systems: Principles, Techniques, Management and Applications; Cambridge: GeoInformation International.

Franck G. (1997): Folgt der Raumordnung die Zeitordnung? Zur technischen Relativisierung von Raum und Zeit In: Schrenk M. (ed): Computergestützte Raumplanung CORP '97. Selbstverlag des Instituts für EDV-gestützte Methoden in Architektur und Raumplanung der TU Wien. (http://www.corp.at)

Frank A.U (1996A): Qualitative Spatial Reasoning: Cardinal Directions as an Example. International Journal of Geographic Information Science JGIS, 10 (3), S.269-290.

Frank A.U (1996B): The prevalence of objects with sharp boundaries in GIS. In: Burrough P.A., Frank A.U. (eds): Geographic objects with indeterminate boundaries GISDATA II [ESF scientific programme on geographic informations systems; data integration and database design] Taylor \& Francis, S.29-40.

Frank A. U. (1997): Ontology: a consumer's point of view. In: Stock O. (ed): Spatial and Temporal Reasoning; Dordrecht, The Netherlands, Kluwer Academic Publishers, S.135-153.

Frank A.U. (1998): Different types of 'times' in GIS. In: Egenhofer, M.J., \& Golledge, R.G. (eds): Spatial and Temporal Reasoning in GIS; Oxford University Press, S.40-62.

Freksa Ch. (1992): Using Orientation Information for Qualitative Spatial Reasoning. In: Frank, A.U., Campari, I., Formentini, U. (eds.): Theories and Methods of Spatio-Temporal Reasoning in Geographic Space; Springer Berlin S.162-178.

Freksa Ch., Barkowski T. (1996): On the Relation between Spatial Concepts and Geographic Objects. In: Burrough P.A., Frank A. (eds): Geographic objects with indeterminate boundaries, GISDATA II, Taylor \& Francis S. 109123.

Freundschuh S.M., Egenhofer M.J. (1997): Human Conceptions of Space: Implications for Geographic Information Systems. Transaction in GIS 2(4), S.361-375.

Friebe T. (1997): Entwurf und Prototyp-Entwicklung eines Java-Applets zur Integration von geographischen Informationen in einen $W W W$-Server. Diplomarbeit, Fachhochschule Köln, Fachbereich Informatik (http://ourworld.compuserve.com/homepages/TFriebe/diplom/welcome.html)

Galton A. (1997): Continuous change in spatial regions. In: Hirtle S.C, Frank A.U. (eds): Spatial information theory, a theoretical basis for GIS, COSIT '97, Springer Verlag, S.1-14.

Geise K., Leuchter S., Müller V., Schmitz D., Poschmann G. (1997): Tutorial zur Einführung in die präskriptive Entscheidungstheorie. Das Zentrum Mensch-Maschine-Systeme (ZMMS) an der Technischen Universität Berlin (http://www.zmms.tu-berlin.de/lehre/tutorial)

Goodchild M. (1992): Integrating GIS and spatial data analysis: problems and possibilities. International Journal of Geographical Information Systems 5/1992, S. 407-423.

Goodchild M.F. (1997): What is Geographic Information Science? NCGIA Core Curriculum in GIScience, Unit 002 (http://www.ncgia.ucsb.edu/giscc/units/u002) 
Goodchild M. F., Egenhofer M.J., Kemp K.K., Mark D. M., Sheppard E. (1999): Introduction to the Varenius project. International Journal of Geographical Information Science 8/1999 (13), S.731-745.

Goodchild M. F. (2000): Spatial analysts and GIS practitioners: The current status of GIS and spatial analysis. Journal of Geographical Systems 2, S.5-10.

Götze U., Bloech J. (1995): Investitionsrechnung : Modelle und Analysen zur Beurteilung von Investitionsvorhaben. Springer Verlag, 2. Auflage.

Goyal R., Egenhofer M. (1997): The Direction-Relation Matrix: A Representation of Direction Relations for Extended Spatial Objects. UCGIS Annual Assembly and Summer Retreat, Bar Harbor, ME.

Goyal R., Egenhofer M. (in press): Cardinal Directions between Extended Spatial Objects. IEEE Transactions on Knowledge and Data Engineering.

Grabaum R., Meyer B.C. (1997): Landschaftsökologische Bewertung und multikriterielle Optimierung mit Geographischen Informationssystemen. Photogrammetrie, Fernerkundung, Geoinformation 2/1997, S.117-130.

Green D. G. (1993): Cellular Automata: Tutorial. Environmental and Information Sciences, Charles Sturt University (http://life.csu.edu.au/complex/tutorials/tutoriall.html)

Greve K., Rinner C. (1999): Argumentationskarten - GIS-basiertes Planungswerzeug im WWW. In: Strobl J., Dollinger F. (eds): Angewandte Geographische Informationsverarbeitung. Beiträge zum AGIT-Symposium Salzburg 1999, H. Wichmann Verlag Heidelberg.

Griess O. (1990): Die Forsteinrichtung, ein klassisches geographisches Informationssystem. Österreichische Forstzeitung 4, S.12-15.

Grimshaw D.J (1994): Using Gis For Strategic Decision Making. Fifth European Conference and Exhibition on Geographical Information Systems EGIS/MARI '94, Utrecht: EGIS Foundation, S.618-625. (http://wwwsgi. ursus.maine.edu/gisweb/spatdb/ egis/eg94070.html)

Güting R.H. (1988): Geo-relational algebra: a model and query language for geometric database systems. In: Schmidt J., Ceri S., Missikoff M. (eds): Advances in database technology - EDBT'88 Italy, Spinger Verlag, S.506-527.

Güting R.H. (1999): Spatial database notes, Tutorial notes. Fachbereich Informatik Praktische Informatik IV Fernuniversität Hagen (http://www.informatik.fernuni-hagen.de/import/pi4/Tutorial-neu.pdf)

Hänle M. (1993): Systeme zur Unterstützung von Gruppenentscheidungen. Konzeption und Implementation eines Prototypen. Dissertation an der Universität - Gesamthochschule Essen, Verlag Josef Eul Bergisch Gladbach Köln.

Hao Q. (1996): The entity-structure-relationship model: towards unified geographic modeling. In: Guan, W., Li, B., Lo, T., Shaw, S.L. \& Zhou, Q. (eds.): GIS \& Remote Sensing: Research, Development \& Applications, The Proceedings of Geoinformatics'96, West Palm Beach, S.79-89.

Harris, Britton, Batty (1992): Locational Models, Geographic Information, and Planning Support Systems. Technical Paper 92-1, National centre for geographic information analysis NCGIA.

Hasebrook, J. (1995): Multimedia-Psychologie: Eine neue Perspektive menschlicher Kommunikation. Spektrum Verlag; Heidelberg, Berlin, Oxford.

Hättenschwiler P. (1997): Informatikvorlesung Datenbanken - Wissensbasierte Systeme. Vorlesungsmaterialien Institute of Informatics, University of Fribourg. (http://www2-iiuf.unifr.ch/ds/courses/db9798)

Hättenschwiller P. (1998): Decision Support Systems. Vorlesung WS 1998/99 Institute of Informatics, University of Fribourg. (http://www-iiuf.unifr.ch/study/courses98-99/DecisionSupport.coursD.html)

Heinimann H.R. (1998): Betrieb und Produktion in der Forstwirtschaft der Zukunft. Forum für Wissen, S.71-77.

Hentschel S. (1999): Funktionsbezogene Optimierung der Walderschließung im Göttinger Stadtwald unter Einsatz Geographischer Informationssysteme. Dissertation an der G. A. Universität Göttingen. (http://webdoc.sub.gwdg.de/diss/1999/hentsche/inhalt.htm)

Hernández D. (1994): Qualitative representation of spatial knowledge. Springer Verlag. 
Hernández D. , Clementini E., Di Felice P. (1995): Qualitative distances. In: Frank A. U., Kuhn W. (eds): Spatial Information Theory: A Theoretical Basis for GIS - International Conference, COSIT'95. Lecture Notes in Computer Science LNCS 988, S.45-57.

Hernández D. (1998): Vorlesungmaterialien: Repräsentation und Verarbeitung räumlichen Wissens. Forschungsund Lehreinheit VII Theoretische Informatik und Grundlagen der KI Fakultät für Informatik TU München. (http://wwwbrauer.informatik.tu-muenchen.de/ danher/danher.html)

Herwijnen v. M., Rietveld P. (1999): Spatial dimensions in multicriteria analysis. In: Thill J.C. (ed): Spatial Multicriteria Decision Making and Analysis: a geographic information sciences approach; Ashgate Verlag, S.77102.

Hong J.-H., Egenhofer M., Frank A. (1995): On the Robustness of Qualitative Distance- and Direction-Reasoning. In: Peuquet D. (ed.): Autocarto 12, Charlotte, NC.

Hornsby K., Egenhofer M., (1997): Qualitative representation of change. In: Hirtle S.C, Frank A.U. (eds): Spatial information theory, a theoretical basis for GIS, COSIT 97, Springer Verlag, S.15-34.

Hornsby K., Egenhofer M. (1998): Identity-Based Change Operations for Composite Objects. In: Poiker T., Chrisman N. (eds.): Eighth International Symposium on Spatial Data Handling, Vancouver, Canada, S.202-213.

Hornsby K., Egenhofer M., Hayes P. (1999): Modeling Cyclic Change. In: Chen P., Embley D., Kouloumdjian J., Liddle S., Roddick J.(eds.): Advances in Conceptual Modeling, Paris, Lecture Notes in Computer Science, Vol. 1227, S.98-109.

Hwang Ch.L., Masud A.S.M. (1979): Multiple objective decision making: methods and applications. Springer Verlag.

Hwang Ch.L., Yoon K. (1981): Multiple attribute decision making: methods and applications. Springer Verlag.

Ingram K., Phillips W. (1987): Geographic Information Processing using a SQL-based Query Language. AutoCarto 8, Baltimore.

Jankowski P. (1995): Integrating geographical information systems and multiple criteria decision making methods. International Journal of Geographical Information Systems 3/1995, S.251-273.

Jankowski P., Lotov A.V., Gusev D. (1999A): Application of a multiple criteria trade-off approach to spatial decision making. In: Thill J.C. (ed): Spatial Multicriteria Decision Making and Analysis: a geographic information sciences approach; Ashgate Verlag, S.127-149.

Jankowski P., Stasik M. (1999B): Design considerations for space and time distributed collaborative spatial decision making. GIDA-Journal of Geographic Information and Decision analysis vol.1, no. 1, S.1-8.

Jansen M., Mues V., Schulz R., Stüber V., Wachter H., Schmidt W., Eberl C., Saborowski J., Sloboda B. (1999): Die Abgrenzung von Wuchsräumen auf der Basis geomorphologischer und klimatologischer Informationslagen. In: Hildebrand E. E.(ed): Von Punkt zur Fläche - Neue Wege, Berichte Freiburger Forstliche Forschung 7, S.39-44.

Jiang H., Eastman J.R. (2000): Application of fuzzy measures in multi-criteria evaluation in GIS. International Journal of Geographic Information Science 14/2000, No. 2, S.173-184.

Kainz W. (1990): Spatial relationships- Topology versus order. In: Brassel K., Kishimoto H. (eds): 4th International Symposium on Spatial Data Handling (SDH) Zürich, S.814-819.

Kainz W., Egenhofer M., Greasley I. (1993): Modeling Spatial Relations and Operations with Partially Ordered Sets. International Journal of Geographical Information Systems 7 (3), S.215-229.

Kammann T. (1999): Qualitative Relationen für Intervalle in verzweigten gerichteten Strukturen. Diplomarbeit, Fachbereich Informatik Universität Hamburg.

Kappel G., Schrefl M. (1996): Objektorientierte Informationssysteme: Konzepte, Darstellungsmittel, Methoden. Springer Verlag Wien.

Keenan P. (1997): Using GIS as a DSS generator. Working paper MIS 95-9, Dep. Of Management Information Systems University College Dublin. 
Kemp Z., Kowalczyk A. (1994): Incorporating the temporal dimension in a GIS. In: Worboys M.F. (ed): Innovations in GIS; Bristol; Taylor \& Francis, Inc., S.89-104.

Keßler U., Schöllmann H. (1998): GIS als Bestandteil eines Forst-Informations-Systems: Der Einsatz von GIS in Forstbetrieben. ArcAktuell 1, S.12.

Koch B., Smaltschinski T. (1997): Geographische Informationssysteme - eine neue Informationstechnologie im Forstbereich. Forst und Holz 7/52, S.176-181.

Kollarits S. (1999): GIS - Applied Methods. Vorlesungsmaterialien Institut für Stadt- und Regionalforschung, TU Wien. (http://esrnt1.tuwien.ac.at/LVA/GIS_Applied/index_gis_applied_vo.html)

Krauß S. (1998): Qualitative Beschreibung von unsicheren topologischen Relationen innerhalb des Minimum/Maximum-Modells. Diplomarbeit Institut für Photogrammetrie Universität Stuttgart.

Kuhn T.S. (1997): Die Struktur wissenschaftlicher Revolutionen. Suhrkamp 14 Auflage (2 erg. Auflage).

Kuhn W. (2000): Wenn sich GIS untereinander nicht verstehen: Fallstudien zur Semantik von Geoinformation. Institut für Geoinformatik Westfälische Wilhelms-Universität Münster. (http://ifgi.unimuenster.de/english/3_projects/sip/presentations)

Kuipers B. (1996): A hierarchy of qualitative representations of space. In: Working papers of the tenth international workshop on quantitative reasoning about physical systems (QR-96) California, American Association for Artificial Intelligence (AAAI) Press.

Kurth H., Gerold D., Ulbricht R. (1994): Forsteinrichtung: nachhaltige Regelung des Waldes. Dt. Landschaftsverlag.

Kwan M.P. (1998): Intermediate GIS: Spatial Decision Making. Vorlesungsmaterialien, G685 SS 98, Department Of Geography Ohio State University. (http://www.geography.ohio-state.edu/classdocs/685Kwan/week10.html)

Laurini R., Thompson D. (1992): Fundaments of spatial information systems. Academic Press London.

Laux H. (1998): Entscheidungstheorie. Springer Verlag.

Lehnhausen (1982): Computergestützte zeitliche und räumliche Simulation forstlicher Systeme an den Beispielen Holzrückeprozess und Materialfluß auf einem Holzhof. Dissertation an der G.A. Universität Göttingen.

Lemm R., Erni V. (1998): Modelle als Hilfsmittel zur Optimierung der Produktionskette. Holz Forum für Wissen, S.51-60.

Leung Y. (1987): On the imprecision of boundaries. Geographical Analysis (19), S.125-151.

Leung (1997): Intelligent Spatial Decision Support Systems. Advances in Spatial Science, Springer Verlag.

Lexer J., Steiner Ch., Vacik H., Lex M. (1997): GIS-gestützte Kartierung der Baumarteneignung für einen Forstbetrieb. In: Strobl J., Dollinger F. (eds): Angewandte Geographische Informationsverarbeitung. Beiträge zum AGIT-Symposium Salzburg 1997, H. Wichmann Verlag Heidelberg.

Lüthy D. (1998): Entwicklung eines 'Spatial Decision Support Systems' (SDSS) für die Holzernteplanung in steilen Geländeverhältnissen. Hochschulverlag an der ETH Zürich, Dissertation.

Maerker L. (1998): Geographische Informationssysteme (GIS). Vorlesungsmaterialien Institut für Geographie, Fakultät für Forst-, Geo-und Hydrowissenschaften, Technische Universität Dresden.

Malczewski J. (1996): A GIS based approch to multiple criteria group decision making. International Journal of Geographic Information Systems 8/1996, S.955 - 971.

Malczewski J, Pazner M., Zaliwska M. (1997): Visualization of multicriteria location analysis using raster GIS: a case study. Carthography and Geographic Information Systems 24/2, S.80-90.

Malczewski J. (1998): Spatial Decision Support Systems. NCGIA Core Curriculum in GIScience (http://www.ncgia.ucsb.edu/giscc/units/u127/u127.html)

Malczewski J. (1999A): GIS and Multicriteria Decision Analysis. John Wiley \& Sons, Inc.

Malczewski J. (1999B): Spatial Multicriteria Decision Analysis. In: Thill J.C. (ed): Spatial Multicriteria Decision Making and Analysis: a geographic information sciences approach, Ashgate Verlag, S.11-48. 
Mandl P. (1994): Räumliche Entscheidungsunterstützung mit GIS: Nutzwertanalyse und FuzzyEntscheidungsmodellierung. In: Dollinger F., Strobl J. (eds) (1994): Angewandte Geographische Informationsverarbeitung VI. Institut für Geographie der Universität Salzburg, Salzburg, (= Salzburger Geographische Materialien, Heft 21), S.463-473.

Mandl P. (1995): Modelling Decision Processes: a New Application Field for the Fuzzy - Systems - Approach. In: Barbic A., Wastl-Walter J. (eds): Sustainable Development of Rural Areas: From Global Problems to Local Solutions. Klagenfurt (= Klagenfurter Geographische Schriften, Heft 13), S.87-94.

Mandl P (1998): Modellierung und Simulation mit GIS: Neue Ansätze durch Fuzzy Set Methodik und Zelluläre Automaten. GeoBit 7/98, S.36-40.

Mark D., Egenhofer M. (1994): Modeling Spatial Relations Between Lines and Regions: Combining Formal Mathematical Models and Human Subjects Testing. Cartography and Geographical Information Systems 21 (3) S.195-212.

Mark D. (1997): Cognitive perspectives on spatial and spatio-temporal reasoning. In: Craglia M., Couclelis H.: Geographic information research, Bridging the atlantic; Taylor\&Francis, S.308-319.

Mark D. (1999A): Workshop Report: Geographic Information Science: Critical Issues in an Emerging CrossDisciplinary Research Domain. Workshop on Geographic Information Science and Geospatial Activities at NSF January 14-15, 1999, (http://www.geog.buffalo.edu/ncgia/workshopreport.html)

Mark D., Freksa Ch., Hirtle S.C., Lloyd R., Tversky B. (1999B): Cognitive models of geographic space. International Journal of Geographical Information Science 8/13, S.747-774.

Mark D. M., Smith B., Tversky B. (1999C): Ontology and Geographic Objects: An Empirical Study of Cognitive Categorisation. In: Freksa C., Mark D. (eds): Spatial Information Theory. International Conference COSIT '99, Lecture Notes in Computer Science, Springer Verlag, S. 283-298.

Mark D. (2000): Geospatial Ontology. UCGIS Annual Assembly and Summer Retreat Meeting Agendas (http://www.ucgis.org/oregon/agendas.html\#resplen)

Matthes F., Schmidt J. W. (1999): Datenbankmodelle und Datenbanksprachen. Buchvorabdruck Department of information and communication technology Technische Universität Hamburg-Harburg. (http://www.sts.tuharburg.de/teaching/ws-99.00/DBIS/entry.html)

Mayer H. (1992): Waldbau auf soziologisch-ökologischer Grundlage. G.Fisher Verlag, 4. Auflage.

Mayer A., Mechler B., Schlindwein A., Wollke R. (1993): Fuzzy Logik: Einführung und Leitfaden zur praktischen Anwendung mit Fuzzy Shell in C++. Addison-Wesley Publishing Company.

Meyer B. C., Grabaum R (1997): Multifunktionale Bewertung und multikriterielle Optimierung von Landschaftsausschnitten. In: Feldmann et al. (eds.): Regeneration und nachhaltige Landnutzung - Konzepte für belastete Regionen. Springer, Heidelberg, S.236-243.

Missler-Behr M.; Lechner W. (1996): Grundelemente der Fuzzy Set Theorie. Arbeitspapiere zur mathematischen Wirtschaftsforschung; Inst. fuer Statistik und Math. Wirtschaftstheorie Universität Augsburg.

Mitasova H., Mitas L. (1998): Process Modeling and Simulations. NCGIA Core Curriculum in Process Modeling and Simulations, NCGIA Core Curriculum in GIScience (http://www.ncgia.ucsb.edu/giscc/units/u130/u130.html)

Montello D. (1997): Human Cognition of the Spatial World. NCGIA Core Curriculum in GIS, National Center for Geographic Information and Analysis, University of California, Santa Barbara, Unit 006 (http://www.ncgia.ucsb.edu/giscc/units/u006/u006.html)

Mues V.: GIS-gestützte Regionalisierung von Klima- und Depositionsdaten in Niedersachsen. Dissertation an der G. A. Universität Göttingen. (http://webdoc.sub.gwdg.de/diss/2000/mues/index.htm)

Mukerjee A.; Hernández D. (1995): Representation of Spatial Knowledge. Tutorial on Fourteenth International Joint Conference on Artificial Intelligence (IJCAI-95). (http://wwwbrauer.informatik.tu-muenchen.de/ danher/ node9.html\#SECTION00022000000)

Nedovic -Budic Z. (1999): Geographic information science implications for urban and regional planning. UCGIS Application White Papers. (http://www.ucgis.org/apps99.html) 
Nyerges T. L. (1990): Spatial databases as models of reality. In: Goodchild, M.F., Kemp K.K.(eds): NCGIA Core Curriculum in GIS UNIT 10. National Center for Geographic Information and Analysis, University of California, Santa Barbara (http://www.geog.ubc.ca/courses/klink/gis.notes/ncgia/ u10.html\#UNIT10)

Nyerges T. L. (1991): Representing geographical meaning. In: Buttenfield B.P., McMaster R.B. (eds): Map generalisation; Making rules for knowledge representation. Longman scientific\&technical S.59-85.

Nyerges T. L. (1996): Interaction Coding Systems for Studying Collaborative Spatial Decision Making. NCGIA Inititive 17 Position Paper. (http://www.ncgia.ucsb.edu:80/research/i17/htmlpapers/nyerges/ Nyerges.html)

Nyerges T. L., Golledge R.G. (1997): Asking Geographic Questions. NCGIA Core Curriculum in GIS, National Center for Geographic Information and Analysis, University of California, Santa Barbara, Unit 007 (http://www.ncgia.ucsb.edu/education/curricula/giscc/units/u007/u007.html)

Okabe A., Miller H.J. (1996): Exact computational methods for calculating distances between objects in a cartographic database. Carthography and Geographic Information Systems 23(4), S.180-195.

Openshaw S., Clarke G. (1996): Developing spatial analysis functions relevant to GIS environments. In: Masser I., Salge F. (eds): Spatial Analytical Perspectives on GIS, GISDATA 4, Taylor\&Francis.

Pacola E. (1999): Optimalizácia lokalizácie trás lesných ciest v prostredí GIS. Autoreferát dizertačnej práce. Dissertation an der Technischen Universität Zvolen.

Papadias, D. (1994): Relation-based representation for spatial knowledge. Dissertation Department of electrical and computer engineering, National Technical University of Athenas.

Papadias D., Egenhofer M.J., Sharma J. (1996A): Hierarchical Reasoning about Direction Relations. In: Shekhar S., P. Bergougnoux (eds.): Proceedings of the 4thACM-GIS, Rockville MD, ACM Press, S.107-114.

Papadias D., Egenhofer M. (1996B): Qualitative collaborative planning in geographic space: some computational issues. NCGIA Initiative 17 Position Papers. (http://www.ncgia.ucsb.edu/research/i17/htmlpapers/ papadias/Papadias.html)

Papadias D., Karacapilidis N., Arkoumanis N. (1999): Processing Fuzzy Spatial Queries: A Configuration Similarity Approach. International Journal of Geographic Information Science, Vol.13(2), S.93-128.

Peuquet D. J.; Ci-Xiang Z. (1987): An algorithm to determine the directional relationship between arbitrarilyshaped polygons in the plane. Pattern recognition 20/1, S.65-74.

Peuquet D. J. (1994): It's about time: A conceptual framework for the representation of temporal dynamics in geographic information systems. Annals of the Association of American Geographers 84(3), S.441-461.

Power D. J. (1997): What is a decision support system. DSstar, The On-Line Executive Journal for Data-Intensive Decision Support, October 21, Vol. 1, No. 3. (http://dss.cba.uni.edu/papers/whatisadss)

Pretzsch H. (1997): Wo steht die Waldwachstumsforschung heute? Denkmuster-Methoden-Feedback. All.Forstu.J.Ztg. 168 (6-7), S.98-102.

Pribbenow S. (1993): Räumliche Inferenz und Bilder. Künstliche Intelligenz 4/93, S.7-14.

Pribbenow S. (1997): What's a part? On formalizing Part-Whole relations. In: Freksa Ch., Jantzen M., Valk R. (eds): Foundations of Computer Science. Potential - Theory - Cognition. Lecture Notes in Computer Science, Springer-Verlag, Berlin.

Pribbenow S. (in press): Parts and Wholes and their Relations. In: Habel Ch., Rickhei G.T. (eds.): Mental Models in Discourse Processing and Problem Solving. John Benjamins Publishing Company, Amsterdam.

Randell D. A. , Cui Z, Cohn A. G. (1992): A Spatial Logic based on Regions and Connection. Proc 3rd Int. Conf on Knowledge Representation and Reasoning, Boston.

Reinberg S., Bröthaler J. (1997): Integration von Fuzzy Methoden in Bewertungsverfahren. In: Schrenk M. (ed): Computergestützte Raumplanung '97. Selbstverlag des Instituts für EDV-gestützte Methoden in Architektur und Raumplanung der Technischen Universität Wien, TU Wien.

Reinberg S. (1998): Räumliche Entscheidungsfindungsprozesse und Modellbildung. In: Strobl J., Dollinger F.: Angewandte Geographische Informationsverarbeitung. Beiträge zum AGIT-Symposium Salzburg 1998. H. Wichmann Verlag Heidelberg. (http://www.geo.sbg.ac.at/agit/papers98/Reinberg/Reinberg.htm) 
Reinberg S. (2000): GIS - Applied Methods. Vorlesungsmaterialien Institut für Stadt- und Regionalforschung, TU Wien (http://esrnt1.tuwien.ac.at/LVA/GIS_Applied/AGis2000/AgisVo)

Rother A. (1998): Ein System zum räumlichen Schließen basierend auf einer objektorientierten Weltrepräsentation. Magisterarbeit in Computerlinguistik und Künstliche Intelligenz, Informatik, Psychologie, Universität Osnabrück (http://www.cl-ki.uni-osnabrueck.de/ arother)

Rottmann M., Schreyer G. (1991): Ein forstliches Geo-Informationssystem für die Bayerische Staatsforstverwaltung. AFZ 14, S.732-736.

Schneeweiss Ch. (1991): Planung 1. Springer Verlag.

Scherrer H.U. (1993): Projekt zur flächenhaften Erfassung und Auswertung von Sturmschäden. AFZ 14, S.712-714.

Schmidt D. (1996): Kopplung des Raster-GIS Idrisi mit einem Fuzzy-Klassifikationsmodell zur Modellierung eines unscharfen räumlichen Entscheidungsprozesses. In: Lorup E., Strobl J. (eds): IDRISI GIS 96 Salzburger Geographische Materialien, Heft 25. Selbstverlag des Instituts für Geographie der Universität Salzburg.

Schmidt D. (1998): Entscheiden(d) in Raumplanungsdiskursen: Interaktive Erstellung und Bewertung digitaler Planungskarten. In: Strobl J., Dollinger F. (eds): Angewandte Geographische Informationsverarbeitung. Beiträge zum AGIT-Symposium Salzburg 1998. H. Wichmann Verlag Heidelberg, Poster.

Schmidtke H. (1998): Forstliche Grundlagenerfassung. ArcAktuell 1, S.23.

Schneider R. (1993): GeoDatenbanksysteme; Eine Speicher- und Zugriffsarchitektur. BI Wissenschaftsverlag, Reihe Informatik.

Schneider M. (1997): Spatial data types for database systems : finite resolution geometry for geographic information systems. Springer Verlag.

Schneider M. (1999): Uncertainty Management for Spatial Data in Databases: Fuzzy Spatial Data Types. In: Gütting R.H. (ed): Advances in Spatial Databases (SSD) 6th Int. Symp., LNCS 1651, Springer Verlag, S.330351.

Schrenk M. (1996): Informationstechnologie als Instrument und als Gegenstand der Raumplanung. In: Schrenk M. (ed): Computergestützte Raumplanung CORP '96. Selbstverlag des Instituts für EDV-gestützte Methoden in Architektur und Raumplanung der TU Wien. (http://osiris.iemar.tuwien.ac.at/ corp/html/inhalt_96.html)

Schulze A., Evers J., Hoppe J., Rumpf S., Meiwes K.J. (1999): Zur Anwendung des Nachhaltigkeitsprinzips im forstlichen Datenmanagement. Forstarchiv 70, S.28-36.

Schut P. H. (1998): Natural Resources Data. NCGIA Core Curriculum in GIScience, Unit 090 (http://www.ncgia.ucsb.edu/giscc/units/u090/u090.html).

Schütz J.Ph. (1999): Die Technik der Waldverjüngung von Wäldern mit Ablösung der Generationen. Skript zur Vorlesung Waldbau II, Professur Waldbau ETH Zürich.

Schwap A. (1994): Einsatz Geographischer Informationssysteme zur Unterstützung politischer Entscheidungsprozesse in der Regionalplanung. In: Dollinger F., Strobl J. (eds): Angewandte Geographische Informationsverarbeitung VI. Institut für Geographie der Universität Salzburg, Salzburg, (= Salzburger Geographische Materialien, Heft 21), S.463-473.

Schwap A. (1995): Einsatz Geographischer Informationssysteme zur Unterstützung politischer Entscheidungsprozesse in der Regionalplanung. In: Dollinger F., Strobl J. (eds): Angewandte Geographische Informationsverarbeitung VI. Institut für Geographie der Universität Salzburg, Salzburg, S.463-473. (http://www.sbg.ac.at/geo/agit/papers95/schwap.htm)

Schwap A. (1996A): GIS, DSS und die Regionalplanung. In: Lorup E., Strobl J. (eds): Idrisi GIS 96. Salzburger Geographische Materialien, Heft 25. Selbstverlag des Instituts für Geographie der Universität Salzburg.

Schwap A. (1996B): Einsatz entscheidungsunterstützender Methoden in der Regionalplanung. In: Schrenk M. (ed): Computergestützte Raumplanung CORP '96. Selbstverlag des Instituts für EDV-gestützte Methoden in Architektur und Raumplanung der TU Wien. (http://osiris.iemar.tuwien.ac.at/ corp/html/inhalt_96.html)

Schwarz-v.Raumer H.G. (1996): Konzept und Anwendung einer GIS-gestützten Modell- und Methodenbank für die raumbezogene Planung. In: Schrenk M. (ed): Computergestützte Raumplanung CORP '96. Selbstverlag des 
Instituts für EDV-gestützte Methoden in Architektur und Raumplanung der TU Wien. (http://www.corp.at/html/schwarz96.html)

Schwarz-v.Raumer, H.G. (1997): GIS-gestützte mehrkriterielle Bewertungsverfahren in der Stadtentwicklungsplanung. GIS Geographische Informationssysteme 6/97, S.13-19.

Sekot W. (1993): Studienunterlagen zur Forsteinrichtung. Schriftenreihe des Instituts für forstliche Betriebswirtschaft und Forstwirtschaftspolitik, Band 19.

Siede W. (1991): Kartierung und Auswertung von Sturmschäden. AFZ 3, S.126-129.

Smith B.,Varzi A.C. (1997): Fiat and bona fide boundaries: Towards an ontology of spatially extended objects. In: Hirtle S.C, Frank A.U.(eds.): Spatial information theory, a theoretical basis for GIS, COSIT 97, Springer Verlag S.103-120.

Smith B., Mark D.M. (1998): Ontology and Geographic Kinds. In: Poiker T. K., Chrisman N. (eds.): Proceedings. 8th International Symposium on Spatial Data Handling (SDH'98), Vancouver: International Geographical Union, S.308-320.

Speidel G. (1972): Planung im Forstbetrieb: Grundlagen und Methoden der Forsteinrichtung. Parey Hamburg.

Spellmann H. (1991): Beiträge der Forsteinrichtung und Ertragskunde für ein forstliches Informationssystem. Forst und Holz 3, S.57-65.

Spitzer H. (1991): Raumnutzungslehre. Stuttgart Ulmer.

Spors H.J., Stock R., Sloboda B. (1992): Räumliche Informationssysteme als Entscheidungsbasis für die forstliche Praxis. Forstarchiv 63, S.33-39.

Spors H.J. (1996): Aufbau und Anwendungsmöglichkeiten eines raumbezogenen Forstlichen Informationssystem. Landesanstalt für Ökologie, Bodenordnung und Forsten/ Landesamt für Agrarordnung NRW, LÖBF Schriftenreihe, Band 7.

Steinmayr T., Auernhammer H., Demmel M. (1998): Sieben Jahre Fahrspuranalyse und Ertragskartierung mit ArcInfo. ArcAktuell 1, S.4.

Stobbe A. (1991): Mikroökonomik. Springer Verlag, 2. Auflage.

Streit U. (1999): Schnupperkurs Geoinformatik. Lehrmaterialien Institut für Geoinformatik Westfälische WilhelmsUniversität Münster. (http://castafiore.uni-muenster.de/vorlesungen/ Schnupperkurs/arbeitsplan.html)

Streit U. (2000): Geoinformatik (v. 2.2). Vorlesungsmaterialien Institut für Geoinformatik der Universität Münster (http://castafiore.uni-muenster.de/vorlesungen/geoinformatik)

Strobl J. (1988): Digitale Forstkarte und Forsteinrichtung : Anwendung von GIS-Technologie in der forstlichen Praxis. Salzburger geographische Materialien; 12.

Strube G. (1995): Kognition. In: Görz G.(ed): Einführung in die künstliche Intelligenz, 2. Aufl., Addison-Wesley, S.299-361.

Strube G. (1996): Wörterbuch der Kognitionswissenschaft. Klett-Cotta Stuttgart.

Stuckenschmidt H., Ranze K. C., Herzog O. (1998): Entwicklungskonzepte für die Wiederverwendung unsicherer Wissensmodelle. TZI-Bericht Nr.12, Technologie-Zentrum Informatik, Universität Bremen, (http://www.informatik.uni-bremen.de/ kcr/paper/TZI/tzi_report.html)

Theodoridis, Y., Papadias, D., Stefanakis, E., Sellis,T (1998): Direction Relations and Two-Dimensional Range Queries: Optimization Techniques. Data \& Knowledge Engineering, vol. 27(3), S.313-336.

Thériault, M., Claramunt, C., Villeneuve, P. (1999): A spatio-temporal taxonomy for the representation of spatial set behaviours. In: Böhlen M., Jensen, Scholl M. (eds.): Spatio-temporal Database Management, SpringerVerlag, S.1-19.

Thill J.C. (1999): Spatial Multicriteria Decision Making and Analysis: a geographic information sciences approach; Ashgate Verlag, S.3-10.

Timpf S. (1998): Hierarchical Structures in map series. Dissertation Department of geoinformation TU Wien (http://www.geoinfo.tuwien.ac.at/publications/timpf/diss/table_of_contents.htm) 
Tomlin D.C. (1990): Geographic information systems and cartographic modeling. Prentice Hall, Englewood Cliffs.

Tränkner H., Siede W. (1989): Das Forstliche Informationssystem FIS. AFZ 40-41, S.1086-1089.

Traub B., Kleinn C. (1997): Quantitative Charakterisierung von Waldflächenformen. Allgemeine Forst- und Jagdzeitung, 1997; 168(2) S.30-39.

Tversky B. (1993): Cognitive maps, cognitive collages, and spatial mental models. In: Frank A.U., Campari I.(eds.): Spatial information theory, a theoretical basis for GIS; COSIT '93 Elba Italy; Springer Verlag, S.14-24.

Unwin D. (1997): GIS and spatial statistical analysis. In: Craglia M., Couclelis H.(eds): Geographic information research, Bridging the atlantic; Taylor\&Francis, S.399-411.

Usery E.L. (1996): A conceptual framework and fuzzy set implementation for geographic features. In: Burrough P.A., Frank A.U.(eds): Geographic objects with indeterminate boundaries GISDATA II [ESF scientific programme on geographic informations systems; data integration and database design] Taylor \& Francis, S.7186.

Vacik H. (1999): Einsatz von GIS und entscheidungsunterstützenden Systemen in der waldbaulichen Planung. Dissertation an der Universität für Bodenkultur Wien.

Vogel M., Blaschke T. (1996): GIS in Naturschutz und Landschaftspflege: Überblick über Wissensstand, Anwendung und Defizite. In: Dollinger F., Strobl J. (eds): Angewandte Geographische Informationsverarbeitung VI. Institut für Geographie der Universität Salzburg, Salzburg, (= Salzburger Geographische Materialien, Heft 21), S.463-473.

Voigt A. (1999): Simulationsgestützte räumliche Planung im Rahmen von Raumplanerausbildung und Forschung an der TU Wien. Computer Aided Planning CAP '99, Oktober 1999 an der TU München. (http://www.isw.de/ cap99/wien_voigt.html)

Vornberger O. (1999): Datenbanksysteme. Vorlesungsmaterialien SS 99 Universität Osnabrück Fachbereich Mathematik/Informatik, 7 Auflage.

Wachowicz M., Healey R.G. (1994): Towards temporality in GIS. In: Worboys M.F. (ed): Innovations in GIS; Bristol; Taylor \& Francis, Inc., S.105-118.

Weichhart P. (1998): Studieninformation Geographie: Am Strand- Eine Einführung in die Weltsicht der Geographie. Department of Geography University Salzburg.

Werner L. (1992): Entscheidungsunterstützungssysteme, Ein problem- und benutzerorientiertes ManagementInstrument. Heidelberg Physica-Verlag.

Winston M. E.,Chaffinn R., Herrmann D. (1987): A taxonomy of part-whole relations. Cognitive Science, 11, S.417- 444 .

Winter S. (1996): Unsichere topologische Beziehungen zwischen ungenauen Flächen. Dissertation, Landwirtschaftliche Fakultät der Rheinischen Friedrich-Wilhelms-Universität Bonn.

Wolf W. (1999): Raumbezogene Bewertungsmöglichkeiten auf der Grundlage von Fuzzy Sets und ihre Implementierung im GIS Spans. Geographisches Institut der Humboldt-Universität Berlin. (http://www2.huberlin.de/geoinf/gk/leute/publik/fuzzy.html)

Worboys M.F., Bofakos P. (1993): A canonical model for a class of areal spatial objects In: Abel D., Ooi B.C. (eds): Advances in Spatial Databases Proceedings of the 3rd International Symposium, SSD93, Springer-Verlag, Berlin S.36-52.

Worboys M.F. (1995): GIS : a computing perspective. Taylor \& Francis.

Yager R. (1988): On Ordered Weighted Averaging aggregation operators in multi-criteria decision making. IEEE Transactions on Systems, Man, and Cybernetics, 8, 183-190.

Yuan M., Albrecht J. (1995): Structural analysis of geographic information and GIS operations from a user's perspective. In: Frank A.U., Kuhn W.(eds.): Spatial information theory, a theoretical basis for GIS; COSIT '95 Austria; Springer Verlag, S.107-122.

Yuan M. (1997): Modelling semantical, spatial band temporal information in a GIS. In: Craglia M., Couclelis H.(eds): Geographic information research, Bridging the atlantic, Taylor\&Francis, S.334-347. 
Yue-Hong Chou (1997): Exploring Spatial Analysis in Geographic Information Systems. OnWord Press Santa Fe USA.

Zimmermann H.J., Gutsche L. (1991): Multi-Criteria Analyse, Einführung in die Theorie der Entscheidungen bei Mehrfachzielsetzungen. Springer Verlag.

Zimmermann K., Freksa Ch. (1993): Qualitatives räumliches Schliessen mit Wissen über Richtungen, Entfernungen und Pfade. Künstliche Intelligenz 4/93, S.21-29. 


\section{Lebenslauf / Curriculum Vitae}

Geburtstag und -ort:

Staatsangehörigkeit:

Familienstand:

$1997-2000$

$1996-1997$

$1990-1996$

(1994-1995)

$1986-1990$

\section{JAROSLAV MYŠIAK}

09.08.1972 in ZILINA/ SLOWAKEI

slowakisch

verheiratet

Doktorstudium an der Fakultät für Forstwissenschaften und Waldökologie in Göttingen

Wissenschaftlicher Mitarbeiter im Institut für Forstliche Biometrie und Informatik Universität Göttingen

Wissenschaftlicher Mitarbeiter im Institut für Forsteinrichtung und Gäodesie an der Forstlichen Fakultät in Zvolen

Studium der Forstwissenschaften an der Forstlichen Fakultät ZVOLEN (SK) und der Fakultät für Forstwissenschaften und Waldökologie GÖTTINGEN

Forstakademie in Liptovsky Hradok (Slowakei)

Göttingen

Februar 2001

Jaroslav Mysiak 